United States Department of Commerce National Institute of Standards and Technology
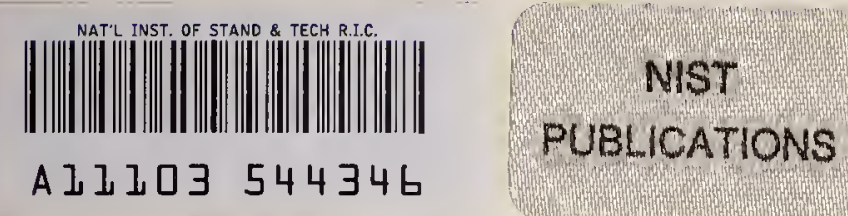

NIST Special Publication 802

\title{
Effect of a Free Surface on Stress Distribution in a Bimaterial Composite
}

V. K. Tewary and R. D. Kriz

QC
100
.$U 57$
$\# 802$
1991
$C .2$


NATIONAL INSTITUTE OF STANDARDS \& TECHNOLOGY

Research Information Center Grithersburg, MD 20099 


\section{Effect of a Free Surface on Stress Distribution in a Bimaterial Composite}

V. K. Tewary* and R. D. Kriz**

Materials Reliability Division

Materials Science and Engineering Laboratory

National Institute of Standards

and Technology

Boulder, Colorado 80303-3328

* On attachment from Ohio State University, Columbus, OH

**Present address: Dept. of Engineering Science and Mechanics,

Virginia Polytechnic Institute and State University, Blacksburg, VA 24061

Issued March 1991

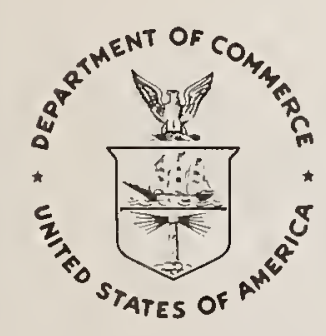

U.S. Department of Commerce

Robert A. Mosbacher, Secretary

National Institute of Standards and Technology

John W. Lyons, Director 
National Institute of Standards and Technology

Special Publication 802

Natl. Inst. Stand. Technol.

Spec. Publ. 802

185 pages (March 1991)

CODEN: NSPUE2
U.S. Government Printing Office

Washington: 1991
For sale by the Superintendent of Documents

U.S. Government Printing Office Washington, DC 20402 
CONTENTS

Page

Preface

Abstract .

PART I

Elastic Green's Function for a Composite solid containing

a Free Surface Normal to the Interface

1. Introduction . . . . . . . . . . . . . . 3

2. Calculation of the SI Green's Function . . . . . . . 3

3. Series Representation of the H-Function. . . . . . . 19

4. Expressions for the Green's Functions. . . . . . . . . 37

5. Application to the Antiplane-Strain Problem. . . . . . 42

6. Application to a Cubic Crystal containing a $\Sigma-5$

Grain Boundary--Plane-strain Problem . . . . . . 53

7. Summary of Main Results. . . . . . . . . . . . 57

Appendix I-A. Elastic Green's Function for an Infinite

Bimaterial composite........ . 58

Appendix I-B. Solution of Generalized Inhomogeneous Vector Hilbert Problem . . . . . . . . 62

Appendix I-C. Evaluation of Integrals . . . . . . . . 72

Figures (Part I) . . . . . . . . . . . 83-93

\section{Part II}

Generalized Plane-Strain Analysis of a Bimaterial Composite Containing a Free Surface Normal to the Interface

1. Introduction . . . . . . . . . . . . . .

2. Displacement and the stress Field in an Infinite Composite Solid subjected to Generalized Plane Strain

3. Displacement and the Stress Field in a Composite Solid Containing a Free Surface. . . . . . 
4. Application to $\Sigma-5$ Grain Boundary in a Cubic Crystal

5. Application to Fiber-Reinforced Laminated Composites

6. Summary of Results and Discussion. . . . . . . .

Appendix II-A. Transform of a Constant and Some Numerical

Figures (Part II). . . . . . . . . . . . 145-174

Acknowledgments. . . . . . . . . . . . . 175

References (For Parts I and II). . . . . . . . . 175 


\section{PREFACE}

The elastic behavior of composite materials is a subject of strong topical interest because of the technological applications of these materials. Since no real solid is infinite, it is important to study the effect of a free surface on the elastic properties of composite materials. A topic of special interest is the stress distribution due to an applied load in such solids. Many papers have already been published on this topic. The behavior of stress near the intersection of the free surface and the interface in a bimaterial composite is singular and quite complex (see, for example, [1-14] and other papers quoted in these references). This is usually referred to as the free-edge effect in composite materials.

The analysis of the free-edge effect is particularly important because damage in a composite material initiates at the free-edges, often as ply cracks and delaminations. Usually, these delaminations are the source of compressive failure in reverse fatigue loading [15]. The free-edge effect is a characteristic property of composite materials and, in general, not exhibited by homogeneous solids. Such a distinction between homogeneous and composite solids should not be surprising because a similar distinction is also found in the behavior of the stress near an interfacial crack tip. Unlike that in a homogeneous solid, the stress near a crack tip, shows strong oscillations $[16,17]$.

There is some confusion in the literature regarding the nature of singularities in the stress field near the free surface. In general it has been found by using different numerical techniques that the stress behaves like $r^{\delta}$ near $r=0$ where $r$ is the radius vector and $\delta$ is a negative number--usually a fraction. 
Zwiers et al. [10] have shown that in case of an out-of-plane load (the generalized plane-strain problem), logarithmic singularities are also present in the stress field. Further, it has been pointed out [10] that additional singularities like $[\log (r)]^{n} \quad(n=1,2,3, \ldots)$ may also arise in the stress field. Moreover, the behavior of the nonsingular terms has not been properly investigated so far except numerically. There is clearly a need for a reliable complete solution which gives the stress distribution in the composite solid containing a free surface.

The stress distribution as well as many other elastic properties of a solid can be easily calculated by using the elastic Green's function of the solid. The Green's function gives the solution of the elastic equilibrium equations for a unit force subject to all the prescribed boundary conditions. Once the Green's function is known, the solution of the elastic equilibrium equations can then be obtained for any arbitrary force distribution by a straightforward integration. The Green's function is therefore a very powerful mathematical tool for calculating the elastic response of a solid.

The objective of the present work is to calculate the elastic Green's function for an anisotropic composite solid containing a free surface and apply it to calculate the stress distribution in the solid to an applied load. We shall consider a semi-infinite bimaterial composite solid containing a planar interface and a single free surface normal to the interface. The solid is assumed to extend to infinity in other directions. This problem will be referred to mathematically as the surface-interface problem and the corresponding Green's function as the surface-interface (abbreviated as the SI) Green's function.

We shall calculate the SI Green's functions for the plane-strain and the generalized plane-strain problems. The out-of-plane components of the strain tensor are zero in the 
plane-strain case and are nonzero but constant in the generalized plane-strain case. The out-of-plane components of the displacement and the stress tensor will be, in general, nonzero, in both cases.

The SI Green's function as calculated here is applicable to different types of bimaterial composites such as, for example, those containing different materials separated by a phase boundary or different orientations of the same crystal separated by a grain boundary. It can also be applied to laminated fiber composites in which different layers have different fiber orientations.

Normally, the Green's function method is used to calculate the stress distribution in a solid due to an specified force. We have, however, extended the Green's function method to the case when instead of a force, an out-of-plane displacement field is specified. This enables us to study the technologically relevant generalized plane-strain problems.

In two earlier papers [18,19], henceforth referred to as I and II, we have calculated the elastic plane-strain Green's function for an infinite bimaterial composite solid. This Green's function gives the solution of the elastic equilibrium equations and satisfies the continuity conditions at the interface. In this paper we shall use this Green's function to calculate the SI Green's function which, in addition, will also satisfy the free surface condition at the free surface.

The calculation of the SI Green's function would require the solution of an inhomogeneous generalized vector Hilbert problem. The properties and the solution of the corresponding scalar problem have been discussed in the excellent treatise by Muskhelishvili [20] and the vector problem by Vekua [21] and also briefly in [20]. However, in the present case, we find it more convenient to solve the aforementioned Hilbert problem by an alternative method using a complex transform. This gives the 
result in a closed integral form which can be calculated quite easily. This method should be generally useful in several problems in stress analysis in composite materials containing cracks, interfaces, and free surfaces.

The earlier methods [1-14] for calculation of the stress distribution near a free surface in a laminated composite are either purely numerical or use a series representation. The series representation is valid only near the origin. Zwiers et al. [10] have used the stroh's method [22] for obtaining a series representation of the stress in terms of the roots of a $12 \times 12$ complex matrix. All these calculations were done for a solid subjected to certain specific loadings.

The purely numerical methods are not reliable near the origin where the stress is singular and quite complex. As pointed out in [10], a series representation obtained by using an eigenfunction expansion method [7] or Lekhnitskii method suffers from lack of completeness of eigenfunction expansion as well as numerical convergence. Moreover, some of these methods do not account for out-of-plane components of the displacement and the stress tensor which, in general, will exist even in plane-strain problems.

The Green's function method can be applied to solids subjected to any loading or force distribution. In addition to the stress distribution, it can be used to calculate the interaction between various defects such as dislocations, surfaces, and cracks. The interaction between defects determines several important physical properties of materials such as, for example, the work hardening of materials.

The Green's function method accounts for the out-of-plane components of the displacement field and the stress tensor in the plane-strain problems. It gives the result in a closed integral 
representation which can be evaluated numerically quite easily. The result is therefore valid for the entire solid and not only near the origin. The integral for the Green's function can also be evaluated analytically, which is useful in identifying the exact nature of all the singularities which may be present in the stress. This method is also numerically more convenient than other methods because it involves manipulation of only a $6 \times 6$ complex matrix and not $12 \times 12$.

This paper is in two parts. In Part I, we have calculated the SI Green's function and applied it to plane-strain problems in cubic solids. Specifically, we have calculated the stress distribution due to a line load in a cubic solid containing a $\Sigma 5$ grain boundary and a free surface normal to the grain boundary interface. Both the plane-strain and antiplane-strain problems have been considered. In Part II, we have described an extension of the Green's function method to the generalized-plane-strain problem which enables us to calculate the elastic response of a solid to a prescribed out-of-plane strain. We have applied it to calculate the stress distribution in a composite solid subjected to an out-of-plane load.

Our final results are in the form of an analytical closed integral representation which can be calculated numerically as well as analytically. The analytical evaluation of the integral by using the contour integration method gives the result in the form of a series. This series contains singular as well as non-singular terms. In general, it is difficult to ascertain the convergence of the series representation. The numerical evaluation of the integral does not suffer from such problems and gives the result to arbitrary accuracy.

The analytical series representation can be used to identify precisely the nature and the weight of each singularity in the stress distribution. This is useful in developing a numerical 
finite-element technique for the stress analysis in a real solid. A purely numerical finite-element technique, when applied to composite materials containing free surfaces, suffers from poor convergence near the origin because of the singularities in the stress. A knowledge of the analytical behavior of the stress near the origin can be used to generate the so-called enriched elements near the origin, which substantially improves the convergence of the numerical technique [14]. 


\section{ABSTRACT}

This paper is in two parts. In part I, the elastic plane-strain Green's function is calculated for an anisotropic bimaterial composite solid containing a free surface normal to the interface. An exact integral representation is obtained for the Green's function which is evaluated numerically. The integral is also evaluated analytically, which gives a series representation for the Green's function. For illustration, the formalism is applied, to the antiplane-strain problem and the plane-strain problem in a cubic solid containing a $\Sigma-5$ grain boundary. In part II, the Green's function is extended to the case of generalized plane strain and applied to calculate the stress field in a cubic solid containing a $\Sigma 5$ boundary and to fiber-reinforced composites with various fiber orientations subjected to an out-of-plane load.

It is found that, as predicted by earlier authors, the stress is singular at the intersection of the free surface and the interface in both plane-strain and generalized plane-strain case. These singularities in the stress field associated with the presence of the free surface are identified and discussed. The singularities in the stress field are found to be of the type $r^{-\delta}$, $\ln (r)$ as well as containing higher powers of $\ln (r)$, where $\delta$ is between 0 and 1 and $r$ is the radial distance from the intersection of the free surface and the interface. In addition, it is found that, in general, the stress field will also contain an oscillatory factor of the type exp[lgln(r)], where $g$ is a constant which depends upon the material parameters of the two solids. However, for the case of the generalized plane strain in fiber-reinforced composites, the factor $g$ is almost 0 so that the oscillatory behavior is absent.

Part I also gives a simple and convenient method for solving an inhomogeneous generalized vector Hilbert problem which contains 
a nonsingular kernel in addition to the usual singular Hilbert kernel. The solution of this Hilbert problem is required for obtaining the Green's function in the present case, as well as many other cases dealing with the stress analysis of composite materials containing surfaces, interfaces, and cracks.

The solution of the Hilbert problem is obtained by using a complex transform of the type $y^{\text {lq-0.5}}$, where $y$ and $q$ are variables on the real axis. It is shown that this function is an eigenfunction of the Hilbert operator and is orthogonal for $y$, varying in the range between 0 and $\pm \infty$ and $q$ between $-\infty$ to $+\infty$. 


\section{PART I}

Elastic Green's Function for a Composite Solid Containing a Free Surface Normal to the Interface

by

V. K. Tewary 



\section{Introduction}

In this part, we describe the calculation of the SI Green's function. We give detailed results for the displacement and the stress Green's function and a discussion of their singularities. For the purpose of illustration, we apply the method to a simple antiplane-strain problem for a cubic solid containing a $\Sigma-5$ grain boundary and also to the corresponding plane strain.

In calculating the SI Green's function, we have used the Green's function for an infinite bimaterial composite as the starting Green's function. A summary of these functions, as taken from two earlier papers $[18,19]$, has been given in Appendix I-A. For calculating the SI Green's function, we need to solve an inhomogeneous generalized vector Hilbert problem. Our method for solving this Hilbert problem has been described in Appendix I-B. The evaluation of certain integrals as required for various formulae in the paper has been described in Appendix I-C.

\section{Calculation of the SI Green's Function}

We consider a bimaterial composite with a planar interface and a free surface normal to the interface as shown in figure I-1. This figure also shows the coordinate system used in our calculations. We take the origin of our coordinate system at the intersection of the free surface and the interface. The X-axis is taken along the interface and the Y-axis is taken along the surface. The Z-axis is taken normal to the plane of paper. We assume that there is no variation of the field quantities (stresses, displacement etc.), which enables us to consider a two-dimensional problem as shown in figure I-1. Although the field quantities will remain constant in the Z-direction, they can and, in general, will have a nonvanishing $\mathrm{Z}$-component. 
The notation in this paper is generally the same as in I and II. The solid in the upper half plane (UHP) and its parameters will be labelled by the superscript $A$ and in the lower half plane (LHP) by the superscript B. The X-, Y-, and $\mathrm{Z}-$ components of a quantity will be identified by subscripts 1,2 , and 3 , respectively. Following the usual notation in the theory of elasticity, we shall denote the radius vector of a point by the vector $\underline{x}$ with its $\mathrm{X}-, \mathrm{Y}-$, and $\mathrm{Z}$ - components denoted by $\mathrm{x}_{1}, \mathrm{x}_{2}$, and $\mathrm{x}_{3}$, respectively. The $\mathrm{x}_{2}$ coordinate may also be represented by $\mathrm{y}$.

The Roman indices $i, j, k$, etc. will take the values 1,2 or 3 and will denote the Cartesian components. Unless otherwise stated, we shall assume the summation convention over repeated Roman indices but not over repeated Greek indices $\alpha, \beta, \lambda$ etc., which will also take the values 1,2 or 3 . We shall denote $\checkmark(-1)$ by Greek $\iota$ and not by the more usual symbol $i$ which, as mentioned earlier, has been used for labelling Cartesian components.

We consider a case when a line force $\underline{\phi}$ is applied to a solid in the XY-plane at the position vector $\underline{x}^{\prime}$. Then the displacement field $\underline{u}(x)$ at point $\underline{x}$ is given by the following equation for elastic equilibrium:

$$
c_{i k j l} \frac{\partial^{2} u_{j}}{\partial x_{k} \partial x_{1}}=\phi_{i} \delta\left(x_{1}-x_{1}^{\prime}\right) \delta\left(x_{2}-x_{2}^{\prime}\right),
$$

where $\subseteq$ denotes the fourth rank elastic constant tensor and $\delta(x)$ is Dirac's delta function. We assume that $\phi$ is applied in the UHP $\left(x_{2}{ }^{\prime}>0\right)$. Equation (1) is written separately for UHP and LHP with $\underline{C}$ and $\underline{u}$ labelled appropriately by $A$ or $B$ for UHP and LHP, respectively. In the equation for LHP, the RHS will be 0 .

We prescribe the following boundary conditions (for $i=1-3$ ): 


$$
\begin{gathered}
u_{i}^{A}\left[x_{1}, 0\right]=u_{i}^{B}\left[x_{1}, 0\right] \quad\left(0 \leq x_{1} \leq \infty\right), \\
\tau_{i 2}^{A}\left[x_{1}, 0\right]=\tau_{i 2}^{B}\left[x_{1}, 0\right] \quad\left(0 \leq x_{1} \leq \infty\right), \\
\tau_{i 1}^{A}\left[0, x_{2}\right]=0 \quad\left(0 \leq x_{2} \leq \infty\right),
\end{gathered}
$$

and

$$
\tau_{i 1}^{B}\left[0, x_{2}\right]=0 \quad\left(0 \geq x_{2} \geq-\infty\right)
$$

where $\underline{\tau}$ is the stress tensor. The stress components $\tau_{i 1}$ and $\tau_{i 2}$ can be obtained from the displacement field by using

$$
\tau_{i 1}=c_{i 11 k} \frac{\partial u_{k}}{\partial x_{1}}+c_{i 12 k} \frac{\partial u_{k}}{\partial x_{2}}
$$

and

$$
\tau_{i 2}=c_{i 21 k} \frac{\partial u_{k}}{\partial x_{1}}+c_{i 2 k} \frac{\partial u_{k}}{\partial x_{2}},
$$

Equations (2) and (3) are the continuity conditions which imply that the two solids are perfectly welded at the interface. Equations (4) and (5) are the free surface conditions at $x_{1}=0$.

our object is to solve eq (1) subject to the boundary conditions given by eqs (2) - (5). The Green's function will be obtained from this solution by taking the force $\phi$ equal to unity. The corresponding Green's function for the case when the unit line force is in LHP can be obtained by replacing $x_{2}^{\prime}$ by $-x_{2}^{\prime}$ and appropriate relabelling of the solids in the UHP and the LHP.

The Green's function, which is a solution of eq (1) and which satisfies the boundary conditions given by eqs (2) and (3), has 
been obtained in I (see also Appendix $A$ of II) and quoted in Appendix I-A of this paper. We shall use this result to obtain the solution of eq (1), which would also satisfy the boundary conditions given by eds (4) and (5).

For this purpose we shall use the standard technique for Green's function calculations (see, for example, I and II) and apply a hypothetical distribution of line forces just outside the region in which the equation has to be solved. We then determine the force distribution by requiring that the solution satisfies the prescribed boundary conditions. In addition, we shall impose the condition that the total space integral of the forces is 0 .

We denote the force distributions by $\underline{F}^{\mathrm{A}}(t)(0 \leq t \leq \infty)$ and $\underline{F}^{B}(t) \quad(-\infty \leq t \leq 0)$ in the UHP and LHP. As shown in figure I-1, these forces are applied just outside the free surface at a continuous set of points in the UHP and LHP viz. $\underline{F}^{A}(t)$ at $x_{1}=-\varepsilon$ and $x_{2}=t \quad(0 \leq t \leq \infty)$ and $\underline{F}^{B}(t)$ at $x_{1}=-\varepsilon$ and $x_{2}=t \quad(-\infty \leq t \leq 0)$, where $\varepsilon$ is a small positive number which is taken to be zero in the limit.

From the definition of the Green's function, we can write the displacement field in UHP and LHP; i.e., a solution of eq (3), in the following form:

$$
\begin{aligned}
\underline{u}^{A}(\underline{x})= & \underline{G}_{0}^{A A}\left(\underline{x} ; \underline{x}^{\prime}\right) \Phi\left(\underline{x}^{\prime}\right)+\int_{0}^{\infty} \underline{G}_{0}^{A A}(\underline{x} ; t) \underline{F}^{A}(t) d t \\
& +\int_{-\infty}^{0} G_{0}^{A B}(\underline{x} ; t) \underline{F}^{B}(t) d t
\end{aligned}
$$

and

$$
\underline{u}^{B}(\underline{x})=\underline{G}_{0}^{B A}\left(\underline{x} ; \underline{x}^{\prime}\right) \Phi\left(\underline{x}^{\prime}\right)+\int_{0}^{\infty} \underline{G}_{0}^{B A}(\underline{x} ; t) \underline{F}^{A}(t) d t
$$

6 


$$
+\int_{-\infty}^{0} \underline{G}_{0}^{B B}(\underline{x} ; t) \underline{F}^{B}(t) d t,
$$

where $G_{0}$ denotes the Green's function for an infinite bimaterial composite which was derived in I. In eqs (8) and (9), we have not explicitly written $-\varepsilon$, the $x_{1}$ component of the position vector of the second variable in the arguments of the Green's functions under the integral signs, for reasons of notational brevity.

It may be emphasized that the so-called force functions $\underline{F}^{A}(t)$ and $\underline{F}^{B}(t)$ are arbitrary functions which have to be determined so that the boundary conditions given by eqs (2)--(5) are satisfied. These functions have the dimensions of force but are not physical forces applied to the solid.

Using the result given in eqs (A.1)-(A.4) of Appendix I-A, we obtain the following expressions for the displacement field from eqs (8) and (9):

$$
\begin{aligned}
\underline{u}^{A}(\underline{x})= & -\frac{1}{\pi} \operatorname{Re} \sum_{\alpha} \underline{\gamma}^{A}(\alpha) \ln \left(z_{\alpha}^{A}-\rho_{\alpha}^{A}\right) \varphi \\
& -\frac{1}{\pi} \operatorname{Re} \sum_{\alpha \beta} \underline{\gamma}^{A}(\alpha) \underline{\Omega}_{\beta}^{I} \ln \left(z_{\alpha}^{A}-\bar{\rho}_{\beta}^{A}\right) \underline{ } \\
& -\frac{1}{\pi} \int_{0}^{\infty} \operatorname{Re}\left[\sum_{\alpha} \underline{\gamma}^{A}(\alpha) \ln \left(z_{\alpha}^{A}+\varepsilon-p_{\alpha}^{A} t\right)\right] \underline{F}^{A}(t) d t \\
& -\frac{1}{\pi} \int_{0}^{\infty} \operatorname{Re}\left[\sum_{\alpha \beta} \underline{\gamma}^{A}(\alpha) \underline{Q}_{\beta}^{I} \ln \left(z_{\alpha}^{A} \varepsilon-\bar{p}_{\beta}^{A} t\right)\right] \underline{F}^{A}(t) d t
\end{aligned}
$$




$$
\begin{array}{r}
+\frac{1}{\pi} \int_{-\infty}^{0} \operatorname{Re}\left[\sum_{\alpha \beta} \underline{\gamma}^{A}(\alpha) \underline{Q}_{\beta}^{I I I} \ln \left(z_{\alpha}^{A}+\varepsilon-p_{\beta}^{B} t\right)\right] \underline{F}^{B}(t) d t \\
\left(\text { for } 0 \leq x_{2} \leq \infty\right)
\end{array}
$$

and

$$
\begin{aligned}
& \underline{u}^{B}(\underline{x})=-\frac{1}{\pi} \operatorname{Re} \sum_{\alpha \beta} \underline{\bar{z}}^{-B}(\alpha) \underline{Q}_{\beta}^{I I} \ln \left(\bar{z}_{\alpha}^{B}-\bar{\rho}_{\beta}^{A}\right) \Phi \\
& -\frac{1}{\pi} \int_{0}^{\infty} \operatorname{Re}\left[\sum_{\alpha \beta} \underline{\bar{\gamma}}^{\mathrm{B}}(\alpha) \underline{\mathrm{Q}}_{\beta}^{I I} \ln \left(\bar{z}_{\alpha}^{\mathrm{B}}+\varepsilon-\bar{p}_{\beta}^{\mathrm{A}} t\right)\right] \underline{F}^{\mathrm{A}}(t) d t \\
& -\frac{1}{\pi} \int_{-\infty}^{0} \operatorname{Re}\left[\sum_{\alpha} \underline{\underline{\gamma}}^{-B}(\alpha) \ln \left(\bar{z}_{\alpha}^{B}+\varepsilon-\bar{p}_{\alpha}^{B} t\right)\right] \underline{F}^{B}(t) d t \\
& +\frac{1}{\pi} \int_{-\infty}^{0} \operatorname{Re}\left[\sum_{\alpha \beta} \underline{\underline{\gamma}}^{\mathrm{B}}(\alpha) \underline{Q}_{\beta}^{I V} \ln \left(\bar{z}_{\alpha}^{\mathrm{B}}+\varepsilon-p_{\beta}^{\mathrm{B}} t\right)\right] \underline{F}^{\mathrm{B}}(t) d t \\
& \left(\text { for }-\infty \leq x_{2} \leq 0\right) \text {, }
\end{aligned}
$$

where $R e$ denotes the real part, overhead bar denotes complex conjugate and other symbols have been defined in Appendix I-A.

Equations (10) and (11) will satisfy eq (1) everywhere in the region of solution, since the line of application of the forces is just outside this region, which is ensured by the presence of $\varepsilon$. In the end we shall take the limit $\varepsilon=0$. 
Further, since the Green's function used in writing eqs (10) and (11) satisfies the continuity conditions at the interface as given by eqs (2) and (3), the solution as given above will also satisfy these conditions for all values of $\underline{F}^{A}(t)$ and $\underline{F}^{B}(t)$. our main task, therefore, is to determine these two force functions so that the free-surface boundary conditions as given by eqs (4) and (5) are also satisfied. The resulting solution will then satisfy all the prescribed boundary conditions.

In addition, we impose the following condition on the force functions to ensure that the displacements are single valued [23] everywhere except possibly at the cut:

$$
\int_{0}^{\infty} \underline{F}^{A}(t) d t+\int_{-\infty}^{0} \underline{F}^{B}(t) d t=0 .
$$

As described in I and II, a simple method for writing the stress component $\tau_{i 1}$ from eqs (8) and (9) is to differentiate the $\log$ functions in these equations with respect to their arguments and replace the matrix $\underline{\gamma}^{\mathrm{A}, \mathrm{B}}$ by the matrix $\underline{\Omega}^{\mathrm{A}, \mathrm{B}}$, where $\underline{\Omega}$ is defined below (for superscripts $A$ or $B$ )

$$
\Omega_{i j}(\alpha)=L_{i k}^{I}(\alpha) \gamma_{k j}(\alpha)
$$

and

$$
L_{i k}^{I}(\alpha)=c_{i 11 k}+p_{\alpha} c_{i 12 k}
$$

The stress components $\tau_{i 2}$ can similarly be obtained by replacing $\underline{\gamma}$ by $\underline{\sigma}$ where $\underline{\sigma}$ has been defined in Appendix I-A. In what follows we shall denote the three components $\tau_{i 1}$ by the vector $\underline{T}_{1}$. Similariy, $\underline{T}_{2}$ will denote the components $\tau_{i 2}$. 
Thus, we obtain the following expressions for $\tau_{i 1}$ from eqs
and (9). (8) and (9).

$$
\begin{aligned}
\underline{T}_{I}^{A}(\underline{x})= & -\frac{1}{\pi} \operatorname{Re} \sum_{\alpha} \underline{\Omega}^{A}(\alpha)\left[z_{\alpha}^{A}-\rho_{\alpha}^{A}\right]^{-1} \Phi \\
& -\frac{1}{\pi} \operatorname{Re} \sum_{\alpha \beta} \underline{\Omega}^{A}(\alpha) \underline{\Omega}_{\beta}^{I}\left[z_{\alpha}^{A}-\bar{\rho}_{\beta}^{A}\right]^{-1} \Phi \\
& +\iota \sum_{\alpha}\left[\underline{J}^{A}(\alpha) \underline{H}^{A}\left(\frac{z_{\alpha}^{A}+\varepsilon}{p_{\alpha}^{A}}\right)+\underline{\bar{J}}^{A}(\alpha) \underline{H}^{A}\left(\frac{\bar{z}_{\alpha}^{A}+\varepsilon}{\bar{p}_{\alpha}^{A}}\right)\right] \\
& +\iota \sum_{\alpha \beta}\left[\underline{K}_{A A}(\alpha, \beta) \underline{H}^{A}\left(\frac{z_{\alpha}^{A}}{\bar{p}_{\beta}^{A}}\right)+\bar{K}_{A A}(\alpha, \beta) \underline{H}^{A}\left(\frac{\bar{z}_{\alpha}^{A}}{p_{\beta}^{A}}\right)\right] \\
& -\iota \sum_{\alpha \beta}\left[\underline{K}_{A B}(\alpha, \beta) \underline{H}^{B}\left(\frac{z_{\alpha}^{A}}{p_{\beta}^{B}}\right)+\underline{\bar{K}}_{A B}(\alpha, \beta) \underline{H}^{B}\left(\frac{\bar{z}_{\alpha}^{A}}{\bar{p}_{\beta}^{B}}\right)\right]
\end{aligned}
$$

and

$$
\begin{aligned}
\underline{T}_{I}^{B}(\underline{x})= & -\frac{1}{\pi} \operatorname{Re} \sum_{\alpha \beta} \underline{\Omega}^{B}(\alpha) \underline{Q}_{\beta}^{I I}\left[\bar{z}_{\alpha}^{B}-\bar{\rho}_{\beta}^{A}\right]^{-1} \Phi \\
& +\iota \sum_{\alpha \beta}\left[\underline{K}_{B A}(\alpha, \beta) \underline{H}^{A}\left(\frac{\bar{z}_{\alpha}^{B}}{\bar{p}_{\beta}^{A}}\right)+\overline{\underline{K}}_{B A}(\alpha, \beta) \underline{H}^{A}\left(\frac{z_{\alpha}^{B}}{p_{\beta}^{A}}\right)\right] \\
& +\iota \sum_{\alpha}\left[\underline{J}^{B}(\alpha) \underline{H}^{B}\left(\frac{z_{\alpha}^{B} \varepsilon}{p_{\alpha}^{B}}\right)+\underline{J}^{B}(\alpha) \underline{H}^{B}\left(\frac{\bar{z}_{\alpha}^{B}+\varepsilon}{\bar{p}_{\alpha}^{B}}\right)\right]
\end{aligned}
$$




$$
-\iota \sum_{\alpha \beta}\left[\underline{K}_{\mathrm{BB}}(\alpha, \beta) \underline{H}^{\mathrm{B}}\left(\frac{\bar{z}_{\alpha}^{\mathrm{B}}}{\mathrm{p}_{\beta}^{\mathrm{B}}}\right)+\overline{\mathrm{K}}_{\mathrm{BB}}(\alpha, \beta) \underline{\mathrm{H}}^{\mathrm{B}}\left(\frac{\mathrm{z}_{\alpha}^{\mathrm{B}}}{\overline{\mathrm{p}}_{\beta}^{\mathrm{B}}}\right)\right],
$$

where

$$
\begin{gathered}
\underline{H}^{A}(z)=\frac{1}{2 \pi \iota} \int_{0}^{\infty} \frac{\underline{F}^{A}(t)}{t-z} d t, \\
\underline{H}^{B}(z)=\frac{1}{2 \pi \iota} \int_{-\infty}^{0} \frac{\underline{F}^{B}(t)}{t-z} d t, \\
\underline{K}_{A A}(\alpha, \beta)=\underline{\Omega}^{A}(\alpha) \underline{\Omega}_{\beta}^{I} / \bar{p}_{\beta}^{A}, \\
\underline{K}_{A B}(\alpha, \beta)=\underline{\Omega}^{A}(\alpha) \underline{\Omega}_{\beta}^{I I I} / p_{\beta}^{B}, \\
\underline{K}_{B A}(\alpha, \beta)=\underline{\bar{\Omega}}^{B}(\alpha) \underline{\Omega}_{\beta}^{I I} / \bar{p}_{\beta}^{A}, \\
\underline{K}_{B B}(\alpha, \beta)=\underline{\bar{\Omega}}^{B}(\alpha) \underline{\Omega}_{\beta}^{I V} / p_{\beta}^{B}, \\
\underline{J}^{A}, B(\alpha)=\underline{\Omega}^{A}, B(\alpha) / p_{\alpha}^{A}, B
\end{gathered}
$$

and $z$, in eqs (17) and (18), is any complex variable. We have neglected $\varepsilon$ in the arguments of the H-functions in eqs (15) and (16) except in the third term on the RHS, because these are the only terms which will be sensitive to $\varepsilon$ in the limit $\varepsilon=0$.

We now equate $\tau_{i 1}^{A}(\underline{x})$ and $\tau_{i 1}^{B}(\underline{x})$ to zero at $x_{1}=0$. At $x_{1}=0$, the variables $z_{\alpha}^{A}$ and $z_{\alpha}^{B}$ assume the following values: 


$$
z_{\alpha}^{A, B}=p_{\alpha}^{A, B} y
$$

where we have denoted the $x_{2}$ - component of $\underline{x}$ by the variable $y$. Using eq (24) in eqs (15) and (16), we obtain the equations

(i) For $0 \leq y \leq \infty$

$$
\begin{aligned}
& \underline{\Gamma}^{A} \underline{H}^{A}(y-\iota \varepsilon)+\underline{\Gamma}^{A} \underline{H}^{A}(y+\iota \varepsilon) \\
& +\sum_{\alpha \beta}\left[\underline{K}_{A A}(\alpha, \beta) \underline{H}^{A}\left(a_{\alpha \beta} Y\right)+\underline{K}_{A A}(\alpha, \beta) \underline{H}^{A}\left(\bar{a}_{\alpha \beta} Y\right)\right] \\
& -\sum_{\alpha \beta}\left[\underline{K}_{A B}(\alpha, \beta) \underline{H}^{B}\left(b_{\alpha \beta} Y\right)+\underline{K}_{A B}(\alpha, \beta) \underline{H}^{B}\left(\bar{b}_{\alpha \beta} Y\right)\right] \\
& =\frac{1}{\pi \iota} \operatorname{Re} \sum_{\alpha} \underline{J}^{A}(\alpha)\left[y-\rho_{\alpha}^{A} / p_{\alpha}^{A}\right]^{-1} \phi \\
& +\frac{1}{\pi \iota} \operatorname{Re} \sum_{\alpha \beta} \underline{P}(\alpha, \beta)\left[y-\bar{\rho}_{\beta}^{A} / p_{\alpha}^{A}\right]^{-1} \phi
\end{aligned}
$$

and

(ii) For $-\infty \leq y \leq 0$

$$
\begin{aligned}
& \sum_{\alpha \beta}\left[\underline{\mathrm{K}}_{\mathrm{BA}}(\alpha, \beta) \underline{\mathrm{H}}^{\mathrm{A}}\left(\overline{\mathrm{d}}_{\alpha \beta} \mathrm{Y}\right)+\underline{\overline{\mathrm{K}}}_{\mathrm{BA}}(\alpha, \beta) \underline{\mathrm{H}}^{\mathrm{A}}\left(\mathrm{d}_{\alpha \beta} \mathrm{Y}\right)\right] \\
& +\underline{\Gamma}^{\mathrm{B}} \underline{\mathrm{H}}^{\mathrm{B}}(\mathrm{Y}-\iota \varepsilon)+\underline{\bar{\Gamma}}^{\mathrm{B}} \underline{\mathrm{H}}^{\mathrm{B}}(\mathrm{y}+\iota \varepsilon)
\end{aligned}
$$




$$
\begin{gathered}
-\sum_{\alpha \beta}\left[\underline{K}_{B B}(\alpha, \beta) \underline{H}^{B}\left(e_{\alpha \beta} Y\right)+\underline{K}_{B B}(\alpha, \beta) \underline{H}^{B}\left(\bar{e}_{\alpha \beta} Y\right)\right] \\
=\frac{1}{\pi \iota} \operatorname{Re} \sum_{\alpha \beta} \underline{R}(\alpha, \beta)\left[y-\bar{\rho}_{\beta}^{A} / \bar{p}_{\alpha}^{B}\right]^{-1} \Phi,
\end{gathered}
$$

where

$$
\begin{aligned}
& \underline{\Gamma}^{\mathrm{A}, \mathrm{B}}=\sum_{\alpha} \underline{\mathrm{J}}^{\mathrm{A}, \mathrm{B}}(\alpha), \\
& \underline{P}(\alpha, \beta)=\underline{J}^{A}(\alpha) Q_{\beta}^{I} \text {, } \\
& \underline{R}(\alpha, \beta)=\underline{\bar{J}}^{\mathrm{B}}(\alpha) \stackrel{\Omega}{\mathrm{II}}_{\beta}^{\mathrm{II}}, \\
& a_{\alpha \beta}=p_{\alpha}^{A} / p_{\beta}^{A}, \\
& \mathrm{~b}_{\alpha \beta}=\mathrm{p}_{\alpha}^{\mathrm{A}} / \mathrm{p}_{\beta}^{\mathrm{B}}, \\
& \mathrm{d}_{\alpha \beta}=\mathrm{p}_{\alpha}^{\mathrm{B}} / \mathrm{p}_{\beta}^{\mathrm{A}}, \\
& e_{\alpha \beta}=\bar{p}_{\alpha}^{B} / p_{\beta}^{B} .
\end{aligned}
$$

In eqs (25) and (26), we have used the fact that, in view of eq (A.20), the sign of the imaginary part of the terms of the type $\varepsilon / p_{\alpha}$ (for superscripts $A$ or $B$ ) will be negative. In the limit $\varepsilon=$ 0 , the functions $H\left(Y \pm \varepsilon / p_{\alpha}\right)$ will be sensitive only to the sign of the imaginary part and not its magnitude, which approaches 0 any way. Accordingly, in the argument of the H-functions, we have written $\varepsilon / p_{\alpha}$ as $-\iota \varepsilon$ and $\varepsilon / \bar{p}_{\alpha}$ as $+\iota \varepsilon$.

It may be remarked that the quantities $\underline{J}, \underline{K}, \underline{\Gamma}, \underline{P}$, etc. (with various subscripts and superscripts) are $3 \times 3$ square matrices, 
whereas $\underline{H}, \underline{F}$, and $\phi$ are $3 \times 1$ column matrices or vectors. Equations (25) and (26) together represent a $6 \times 6$ matrix equation or a set of 6 simultaneous equations in 6 unknowns. The unknowns are the three components each of $\underline{F}^{A}(t)$ and $\underline{F}^{B}(t)$. These equations constitute a generalized inhomogeneous vector Hilbert problem. Its solution has been described in Appendix I-B.

Following the method described in Appendix I-B, we write the two force functions in terms of their complex transforms as given by eqs (B.25) and (B.26). In Appendix I-B, the functions $\underline{F}(t)$ and $\underline{H}(z)$ are assumed to be vectors having two components. In the present case, these are vectors having 6 components. However, the formulae given in Appendix I-B are formally valid in the present case as well, provided we regard $\underline{F}^{A}(t), \underline{F}^{B}(t), \underline{H}^{A}(z)$, and $\underline{H}^{B}(z)$ themselves as 3 component vectors.

Thus, as in eqs (B.27) and (B.28), we write the following representation for the $\mathrm{H}$-functions:

and

$$
\underline{H}^{A}(z)=\int_{-\infty}^{\infty} \underline{v}^{A}(q) z^{l q-0.5} d q
$$

$$
\underline{H}^{B}(z)=\int_{-\infty}^{\infty} \underline{v}^{B}(q) z^{l q-0.5} d q,
$$

where $\underline{v}^{A}(q)$ and $\underline{v}^{B}(q)$ are the unknown functions of the real variable variable $q$, which have to be determined. For each $q$, these are $3 \times 1$ column matrices like the H-functions.

Once the $\mathrm{H}$ functions are known, the force functions can be obtained in a simple manner in terms of the discontinuity in the $\mathrm{H}$ function over the real axis by using the Plemelj relation (see, for example, [20]) as given by eq (B.42). 
We now substitute for the $\mathrm{H}$ functions from eqs (34) and (35) in eqs (25) and (26) and use the method described in Appendix I-B for deriving eqs (B.31) and (B.32). This would transform each element of the matrices in eqs (25) and (26) into a function of $q$ alone. As remarked earlier, we can represent eqs (25) and (26) by a single $6 \times 6$ matrix equation. Accordingly, we regard $\underline{V}(q)$ as a single $6 \times 1$ column matrix or a six-dimensional vector. Thus, eqs (25) and (26), after the transformation can be written in the following matrix representation:

$$
\underline{M}(q) \underline{V}(q)=\underline{N}(q) / E(q) \text {, }
$$

where $\underline{M}(q)$ and $N(q)$ are respectively the $6 \times 6$ and $6 \times 1$ matrices formed by the transformed elements of the LHS and RHS of eqs (25) and (26).

In order to represent the matrices $\underline{M}(q), \underline{N}(q)$ and $\underline{V}(q)$, it would be convenient to introduce a block matrix notation in terms of $A$ and $B$ which correspond to the solids in UHP and LHP respectively. According to the block scheme, we first write the $6 \times 1$ matrix $\underline{\mathrm{V}}(\mathrm{q})$ in terms of two $3 \times 1$ blocks $\underline{\mathrm{V}}^{\mathrm{A}}(q)$ and $\underline{\mathrm{V}}^{\mathrm{B}}(\mathrm{q})$ and then write $\underline{M}(q)$ and $\underline{N}(q)$ in terms of the same block representation. Their matrix elements will thus be denoted in terms of the block matrices $\underline{M}_{A B}, M_{A A}, N_{A}$, etc. For notational brevity, we shall not show their functional dependence on $q$ except where explicitly needed.

The block structure of eq (36) can thus be written as

$$
\left(\begin{array}{ll}
\underline{M}_{A A} & \underline{M}_{A B} \\
\underline{M}_{B A} & \underline{M}_{B B}
\end{array}\right)\left(\begin{array}{l}
\underline{v}^{A}(q) \\
\underline{v}^{B}(q)
\end{array}\right)=\frac{1}{E(q)}\left(\begin{array}{l}
\underline{N}_{A} \\
\underline{N}_{B}
\end{array}\right) \text {, }
$$

where 


$$
\begin{aligned}
& \underline{M}_{A A}=-\underline{\Gamma}^{A} \exp (-2 \pi q)+\underline{\bar{\Gamma}}^{A} \\
& +\sum_{\alpha \beta}\left[\underline{\mathrm{K}}_{\mathrm{AA}}(\alpha, \beta)\left[\mathrm{a}_{\alpha \beta}\right]^{\mu}+\underline{\underline{\mathrm{K}}}_{\mathrm{AA}}(\alpha, \beta)\left[\overline{\mathrm{a}}_{\alpha \beta}\right]^{\mu}\right] \text {, } \\
& \underline{\underline{M}}_{\mathrm{AB}}=-\sum_{\alpha \beta}\left[\underline{\mathrm{K}}_{\mathrm{AB}}(\alpha, \beta)\left[\mathrm{b}_{\alpha \beta}\right]^{\mu}+\overline{\underline{\mathrm{K}}}_{\mathrm{AB}}(\alpha, \beta)\left[\overline{\mathrm{b}}_{\alpha \beta}\right]^{\mu}\right], \\
& \underline{M}_{\mathrm{BA}}=\sum_{\alpha \beta}\left[\underline{\mathrm{K}}_{\mathrm{BA}}(\alpha, \beta)\left[-\overline{\mathrm{d}}_{\alpha \beta}\right]^{\mu}+\overline{\underline{\mathrm{K}}}_{\mathrm{AB}}(\alpha, \beta)\left[-\mathrm{d}_{\alpha \beta}\right]^{\mu}\right], \\
& \underline{M}_{\mathrm{BB}}=\iota \underline{\Gamma}^{\mathrm{B}} \exp (\pi q)-\iota \underline{\Gamma}^{\mathrm{B}} \exp (-\pi q) \\
& -\sum_{\alpha \beta}\left[\underline{\mathrm{K}}_{\mathrm{BB}}(\alpha, \beta)\left[-\mathrm{e}_{\alpha \beta}\right]^{\mu}+\overline{\underline{\mathrm{K}}}_{\mathrm{BB}}(\alpha, \beta)\left[-\overline{\mathrm{e}}_{\alpha \beta}\right]^{\mu}\right] \text {, } \\
& \underline{N}^{A}=\frac{\exp (-2 \pi q)}{2 \pi} \sum_{\alpha}\left[\underline{J}^{A}(\alpha)\left(\rho_{\alpha}^{A} / p_{\alpha}^{A}\right)^{\bar{\mu}}+\underline{\bar{J}}^{A}(\alpha)\left(\bar{\rho}_{\alpha}^{A} / \bar{p}_{\alpha}^{A}\right)^{\bar{\mu}}\right] \Phi \\
& +\frac{\exp (-2 \pi q)}{2 \pi} \sum_{\alpha \beta}\left[\underline{P}(\alpha, \beta)\left(\bar{\rho}_{\beta}^{\mathrm{A}} / \mathrm{p}_{\alpha}^{\mathrm{A}}\right)^{\bar{\mu}}+\underline{\overline{\mathrm{P}}}(\alpha, \beta)\left(\rho_{\beta}^{\mathrm{A}} / \overline{\mathrm{p}}_{\alpha}^{\mathrm{A}}\right)^{\bar{\mu}}\right] \Phi \text {, }
\end{aligned}
$$

$$
E(q)=1+\exp (-2 \pi q),
$$

and

$$
\mu=\iota q-0.5 .
$$


Using the same representation as given in eq (37), the block structure of the matrix $\underline{H}(z)$ is

$$
\underline{H}(z)=\left(\begin{array}{c}
\underline{H}^{A}(z) \\
\underline{H}^{B}(z)
\end{array}\right) \text {. }
$$

Equation (37) can be solved for $\underline{\mathrm{V}}(\mathrm{q})$ by matrix inversion:

$$
\underline{V}(q)=\underline{M}^{-1} \underline{N} / E(q) \text {. }
$$

For later use, we shall also write eq (47) in block representation corresponding to eq $(36)$ as

$$
\left(\begin{array}{c}
\underline{v}^{A}(q) \\
\underline{v}^{B}(q)
\end{array}\right)=\frac{1}{E(q) D(q)}\left(\begin{array}{ll}
\underline{c}_{A A} & \underline{c}_{A B} \\
\underline{c}_{B A} & \underline{c}_{B B}
\end{array}\right)\left(\begin{array}{l}
\underline{N}_{A} \\
\underline{N}_{B}
\end{array}\right) \text {, }
$$

where $\underline{C}$ are the block elements of the matrix of cofactors of $\underline{M}$ in the same block representation and $D(q)$ is the determinant of $\underline{M}$.

The matrix of cofactors is proportional to the inverse of $\mathrm{M}$ which, in the present block representation, can be formally expressed as

$$
\sum_{\mathrm{a}} \underline{\mathrm{C}}_{\mathrm{Aa}}(\mathrm{q}) \underline{\mathrm{M}}_{\mathrm{aB}}(\mathrm{q})=\sum_{\mathrm{a}} \underline{\mathrm{M}}_{\mathrm{Aa}}(q) \underline{\mathrm{C}}_{\mathrm{aB}}(\mathrm{q})=\mathrm{D}(\mathrm{q}) \delta_{\mathrm{AB}}
$$

where $\delta_{A B}$ is Kronecker's delta and $A, a$, and $B$ are block indices which label the blocks in the corresponding matrix representation. As in the case of $\underline{M}$ and $\underline{N}$ matrices, we shall show the functional dependence of the elements of $\underline{C}$ on $q$ only where explicitly needed. 
In general the inverse of $\underline{M}$ in eq (48) has to be obtained numerically, and there is no need to calculate the cofactor matrix. The two $\mathrm{H}$ functions can then be calculated from eqs (34) and (35) by integrating over $q$. The integrals over $q$ in these equations can be calculated numerically in a straightforward manner. It is also possible to evaluate the $q$ integral analytically by using contour integration. For this purpose eq (49) is more convenient. This would give a series representation of the H-functions, which will be derived in the next section.

Equation (47) gives the particular solution of Hilbert's equation. To obtain the general solution of Hilbert's equation, as described in Appendix I-B, we need to add the following homogeneous solution

$$
\underline{H}_{0}(z)=\sum_{r} \underline{h}\left(Q_{r}\right) z^{L Q_{r}-0.5},
$$

where $Q_{r}$ is a root of the determinantal equation

$$
D\left(Q_{r}\right)=0,
$$

and $\underline{h}\left(Q_{r}\right)$ is an eigenvector of $\underline{M}(q)$ corresponding to zero eigenvalue for $q=Q_{r} ;$ it is a solution of the matrix equation:

$$
\underline{M}\left(Q_{r}\right) \underline{h}\left(Q_{r}\right)=0
$$

In analogy with vectors $\underline{H}(z)$ and $\underline{V}(q)$ the two block components of $\underline{h}\left(Q_{r}\right)$ will be labelled by the superscripts $A$ and $B$. According to the theory of homogeneous equations, one of the components of $\underline{h}$ will be arbitrary. This provides a set of arbitrary constants (one for each value of $Q_{r}$ ), which have to be chosen so that eq (12) is satisfied. These constants can also be chosen to satisfy any other boundary condition on the stress field 
such as those which may be required at the outer surfaces in a finite solid.

Thus, finally, we can write the H- functions which give the complete solution of eqs (25) and (26) as follows:

$$
\underline{H}(z)=\left(\begin{array}{c}
\underline{H}_{p}^{A}(z) \\
\underline{H}_{-p}^{B}(z)
\end{array}\right)+\left(\begin{array}{c}
\underline{H}_{0}^{A}(z) \\
\underline{H}_{0}^{B}(z)
\end{array}\right),
$$

where the subscript $\mathrm{p}$ denotes the particular solution which is given by eqs (34), (35) and (47). The homogeneous solution, labelled with the subscript 0 , is given by eq (50).

Henceforth, for notational brevity, we shall omit the subscript $p$ from the particular solution except where we need to distinguish it from the homogeneous solution. Accordingly, $\underline{H}(z)$ will refer to the particular solution for the H-function.

The expression for the H-functions as given above is the main result of this paper. The stress distribution in the solid is given by eqs (15) and (16) in terms of the two H-functions. The displacement field is given by eqs (10) and (11) in terms of the logarithmic integrals which can be obtained from the $\mathrm{H}$ - functions by a simple integration over $z$. Equations (10) and (11) give the required Green's function when $\phi$ is taken to be a unit force.

In the next section, we shall derive a series representation for the $\mathrm{H}$-functions and discuss their singularities.

\section{Series Representation of the H-Function}

In the previous section we obtained the two $H$ functions $\underline{H}^{A}$ and $\underline{H}^{B}$, as integrals over $q$. In this section we shall obtain 
series representations for $\underline{H}^{A}$ and $\underline{H}^{B}$ in the Cartesian space and discuss their singularities. The singularities in the H-functions give the singularities in the stress. We shall also obtain the space integral of the force functions $\underline{F}^{A}$ and $\underline{F}^{B}$ in order to ensure that eq (12) is satisfied.

First we evaluate $\underline{H}^{A}(z)$ and $\underline{H}^{B}(z)$ from eqs (34) and (35) for a complex variable $z$. Using eq $(48)$, we write $\underline{H}^{A}(z)$ in the form

$$
\underline{H}^{A}(z)=\int_{-\infty}^{\infty} \frac{\underline{p}^{A}(q) z^{i q-0.5} d q}{E(q) D(q)}
$$

where

$$
\underline{P}^{A}(q)=\left[\underline{C}_{A A}(q) N_{A}(q)+\underline{C}_{A B}(q) \quad N_{B}(q)\right]
$$

with a similar expression for $\underline{H}^{B}(z)$.

The q-integral in eq (54) can be evaluated by using the method of contour integration as described in Appendix I-C for eq (C.16). From the result given by eq (C.24) we obtain the following expressions for $\underline{H}^{A}(z)$ and $\underline{H}^{B}(z)$ (in the limit $\varepsilon=0$ )

$$
\begin{aligned}
& \underline{H}^{A}(z)=\sum_{\alpha}\left[\sum_{n} \Phi_{A A}(n, \varepsilon) z^{-(n+1)-\varepsilon} \zeta_{1}(\alpha, n)\right. \\
& \left.\quad+\sum_{r} \underline{\Psi}_{A A}(r, \varepsilon) z^{\iota Q_{r}-0.5+\varepsilon} \xi_{1}\left(\alpha, \iota Q_{r}\right)\right] s_{z}
\end{aligned}
$$




$$
\begin{aligned}
& +\sum_{\alpha \beta}\left[\sum_{n} \Phi_{A A}(n, \varepsilon) z^{-(n+1)-\varepsilon} \zeta_{2}(\alpha \beta, n)\right. \\
& \left.+\sum_{r} \Psi_{A A}(r, \varepsilon) z^{\iota Q_{r}-0.5+\varepsilon} \xi_{2}\left(\alpha \beta, \iota Q_{r}\right)\right] s_{z} \\
& +\sum_{\alpha \beta}\left[\sum_{n} \Phi_{A B}(n, \varepsilon) z^{-(n+1)-\varepsilon} \zeta_{3}(\alpha \beta, n)\right. \\
& \left.+\sum_{r} \underline{\Psi}_{A B}(r, \varepsilon) z^{\iota Q_{r}-0.5+\varepsilon} \xi_{3}\left(\alpha \beta, \iota Q_{r}\right)\right] s_{z}+R_{H}^{A}(z)
\end{aligned}
$$

and

$$
\begin{aligned}
\underline{\mathrm{H}}^{\mathrm{B}}(\mathrm{z})= & \sum_{\alpha}\left[\sum_{\mathrm{n}} \underline{\Phi}_{\mathrm{BA}}(\mathrm{n}, \varepsilon) \mathrm{z}^{-(\mathrm{n}+1)-\varepsilon} \zeta_{1}(\alpha, n)\right. \\
& \left.+\sum_{\mathrm{r}} \underline{\Psi}_{\mathrm{BA}}(\mathrm{r}, \varepsilon) \mathrm{z}^{\iota Q_{\mathrm{r}}-0.5+\varepsilon} \xi_{1}\left(\alpha, \iota Q_{r}\right)\right] s_{z} \\
& +\sum_{\alpha \beta}\left[\sum_{\mathrm{n}} \underline{\Phi}_{\mathrm{BA}}(\mathrm{n}, \varepsilon) \mathrm{z}^{-(n+1)-\varepsilon} \zeta_{2}(\alpha \beta, n)\right.
\end{aligned}
$$




$$
\begin{aligned}
& \left.+\sum_{r} \Psi_{B A}(r, \varepsilon) z^{\iota Q_{r}-0.5+\varepsilon} \xi_{2}\left(\alpha \beta, \iota Q_{r}\right)\right] s_{z} \\
& +\sum_{\alpha \beta}\left[\sum_{B B}(n, \varepsilon) z^{-(n+1)-\varepsilon} \zeta_{3}(\alpha \beta, n)\right. \\
& \left.+\sum_{r} \underline{\Psi}_{B B}(r, \varepsilon) z^{\iota Q_{r}-0.5+\varepsilon} \xi_{3}\left(\alpha \beta, \iota Q_{r}\right)\right] s_{z}+R_{H}^{A}(z),
\end{aligned}
$$

where Re denotes the real part and other symbols are as defined below:

$$
\begin{gathered}
\Phi_{A A}(n, \varepsilon)=-\iota \exp (-2 \pi \iota \varepsilon) \underline{c}_{A A}\left(q_{n}+\iota \varepsilon\right) / D\left(q_{n}+2 \iota \varepsilon\right), \\
\Phi_{A B}(n, \varepsilon)=\iota(-1)^{n} \exp (-\pi \iota \varepsilon) \underline{c}_{A B}\left(q_{n}+\iota \varepsilon\right) / D\left(q_{n}+2 \iota \varepsilon\right), \\
\Phi_{B A}(n, \varepsilon)=-\iota \exp (-2 \pi \iota \varepsilon) \underline{c}_{B A}\left(q_{n}+\iota \varepsilon\right) / D\left(q_{n}+2 \iota \varepsilon\right), \\
\Phi_{B B}(n, \varepsilon)=\iota(-1)^{n} \exp (-\pi \iota \varepsilon) \underline{c}_{B B}\left(q_{n}+\iota \varepsilon\right) / D\left(q_{n}+2 \iota \varepsilon\right), \\
\Psi_{A A}(r, \varepsilon)=\frac{2 \pi \iota \exp \left[-2 \pi\left(Q_{r}-\iota \varepsilon\right)\right] \underline{C}_{A A}\left(Q_{r}-\iota \varepsilon\right)}{E\left(Q_{r}-2 \iota \varepsilon\right) D^{\prime}\left(Q_{r}\right)}, \\
\underline{\Psi}_{A B}(r, \varepsilon)=-\frac{2 \pi \exp \left[-\pi\left(Q_{r}-\iota \varepsilon\right)\right] \underline{C}_{A B}\left(Q_{r}-\iota \varepsilon\right)}{E\left(Q_{r}-2 \iota \varepsilon\right) D^{\prime}\left(Q_{r}\right)},
\end{gathered}
$$




$$
\begin{aligned}
& \Psi_{B A}(r, \varepsilon)=\frac{2 \pi \iota \exp \left[-2 \pi\left(Q_{r}-\iota \varepsilon\right)\right] \underline{C}_{B A}}{E\left(Q_{r}-2 \iota \varepsilon\right) D^{\prime}\left(Q_{r}\right)}, \\
& \underline{\Psi}_{B B}(r, \varepsilon)=-\frac{2 \pi \exp \left[-\pi\left(Q_{r}-\iota \varepsilon\right)\right] \underline{C}_{B B}\left(Q_{r}-\iota \varepsilon\right)}{E\left(Q_{r}-2 \iota \varepsilon\right) D^{\prime}\left(Q_{r}\right)}, \\
& \zeta_{1}(\alpha, n)=2 \operatorname{Re} \underline{J}^{A}(\alpha)\left[\rho_{\alpha}^{A} / p_{\alpha}^{A}\right]^{n+\varepsilon} \\
& \xi_{1}\left(\alpha, \iota Q_{Y}\right)=I^{A}(\alpha)\left[\rho_{\alpha}^{A} / p_{\alpha}^{A}\right]^{-\iota Q_{r}-0.5-\varepsilon}+ \\
& +\underline{\mathrm{J}}^{A}(\alpha)\left[\bar{\rho}_{\alpha}^{A} / \overline{\mathrm{p}}_{\alpha}^{A}\right]^{-\iota Q_{r}-0.5-\varepsilon} \\
& \zeta_{2}(\alpha \beta, n)=2 \operatorname{Re} \underline{P}(\alpha, \beta)\left[\bar{\rho}_{\beta}^{-A} / p_{\alpha}^{A}\right]^{n+\varepsilon} \text {, } \\
& \xi_{2}\left(\alpha \beta, \iota Q_{r}\right)=\underline{P}(\alpha, \beta)\left[\bar{\rho}_{\beta}^{A} / p_{\alpha}^{A}\right]^{-\iota Q_{r}-0.5-\varepsilon} \\
& +\underline{\bar{P}}(\alpha, \beta)\left[\rho_{\beta}^{A} / \bar{p}_{\alpha}^{-A}\right]^{-l Q_{r}-0.5-\varepsilon} \\
& \zeta_{3}(\alpha \beta, n)=2 \operatorname{Re} \underline{R}(\alpha, \beta)\left[\bar{\rho}_{\beta}^{-A} / \bar{p}_{\alpha}^{-B}\right]^{n+\varepsilon}, \\
& \xi_{3}\left(\alpha \beta, \iota Q_{r}\right)=\underline{R}(\alpha, \beta)\left[\bar{\rho}_{\beta}^{-A} / \bar{p}_{\alpha}^{-B}\right]^{-\iota Q_{r}-0.5-\varepsilon}
\end{aligned}
$$

23 


$$
+\underline{\bar{R}}(\alpha, \beta)\left[\rho_{\beta}^{\mathrm{A}} / \mathrm{p}_{\alpha}^{\mathrm{B}}\right]^{-\iota Q_{Y}-0.5-\varepsilon}
$$

The vectors $\zeta_{1}, \zeta_{2}, \zeta_{3}, \xi_{1}$, and $\xi_{2}$ are in units of $\phi / 2 \pi$. The factor $s_{z}$ is +1 if the integration contour was chosen to be in the UHP (that is, if the expansion parameter is greater than 1) and -1 otherwise. In deriving the above equations, we have taken the following values of the branches of the complex exponential

$$
\exp (\pi \iota / 2)=\iota \text { and } \exp (-\pi \iota / 2)=-\iota \text {. }
$$

As described in Appendix I-C [see eqs (C.22) and (C.23)], the allowed values of $\mathrm{n}$ and $Q_{r}$ in each sum depend upon the magnitude of the expansion variable. For example in the first term on the RHS of eq (61), n will take 0 or any positive integral value if $\bmod \left(z \mathrm{p}_{\alpha}^{\mathrm{A}} / \rho_{\alpha}^{\mathrm{A}}\right)$ is larger than unity and negative integral values otherwise. Thus $n$ and also of course $Q_{r}$ depend upon the indices of the outer sum. The factor $s_{z}$ in eqs (56) and (57), which defines the sign of each expansion term, also depends upon the indices of the outer sum. Because of this dependence we cannot write the above equations in a compact form for a general value of $z$.

However, in the two limiting cases when mod $(z) \rightarrow 0$ or mod $(z) \rightarrow \infty, n$ as well as $Q_{r}$ become independent of $\alpha$ or $\beta$. In such cases the above equations are considerably simplified. Since these cases are of substantial interest, we give below the matrix representations for the two $H$ functions in the $A B$ block representation in the two limiting cases:

$$
\begin{aligned}
& \underline{H}(z)= \pm \sum_{n} \Phi(n, \varepsilon) \quad \zeta(n, \varepsilon) z^{-(n+1)-\varepsilon} \\
& \pm \sum_{Y} \underline{\Psi}\left(Q_{r^{\prime}} \varepsilon\right) \xi\left(\iota Q_{r^{\prime}} \varepsilon\right) z^{\iota Q_{Y}-0.5+\varepsilon} \pm \mathrm{R}_{H}(z),
\end{aligned}
$$


where $\Phi$ and $\underline{\Psi}$ are square matrices, and $\underline{H}, \underline{R}_{H}, \zeta$ and $\xi$ are column vectors with elements labelled by $A$ and $B$ in the same block representation as defined by eq (37). As in eq (C.24), the plus sign is to be taken if $\bmod (z)>1$ and negative if $\bmod (z)<1$. The matrix elements of $\Phi$ and $\underline{\Psi}$ have already been defined in eqs (58) $--(65)$. The elements of $\zeta$ and $\xi$ are defined as

$$
\begin{gathered}
\left.\zeta_{A}(n)=\sum_{\alpha} \zeta_{1}(\alpha, n)+\sum_{\alpha \beta} \zeta_{2}(\alpha \beta, n)\right], \\
\zeta_{B}(n)=\sum_{\alpha \beta} \zeta_{3}(\alpha \beta, n), \\
\xi_{A}\left(\left\llcorner Q_{r}\right)=\sum_{\alpha} \xi_{1}\left(\alpha, \iota Q_{r}\right)+\sum_{\alpha \beta} \xi_{2}\left(\alpha \beta, \iota Q_{r}\right),\right. \\
\xi_{B}\left(\left\llcorner Q_{r}\right)=\sum_{\alpha \beta} \xi_{3}\left(\alpha \beta, \iota Q_{r}\right) .\right.
\end{gathered}
$$

The dependence of the various parameters in eqs $(74)-(77)$ on $\varepsilon$ is not shown for reasons of notational brevity. As described in Appendix I-C, $\varepsilon$ can be set to zero immediately for those values of $\mathrm{n}$ and $\mathrm{r}$ for which there are no higher order poles. When the higher order poles are present, their contribution has to be calculated separately by evaluating the limit at $\varepsilon=0$. In eq (73), this contribution has been denoted by $R_{H}(\underline{z})$. Equations (73), (56) and (57), therefore, provide a convenient representation for the $\mathrm{H}$ functions which is formally valid whether higher order poles are present or not. In section 5 we shall consider the antiplane-strain case in which all $\mathrm{q}_{n}$ 's are also roots of eq (51) and are therefore second-order poles.

As explained in Appendix I-C and given by eq (C.22) and (C.23), the position and the contribution of poles depend upon whether the contour of integration has been chosen in the UHP or LHP. This, in turn, depends upon the magnitude of $z$ or the 
expansion variable. In sections $3 B$ and $3 C$, respectively, we have calculated the contribution of the poles in the two limiting cases when $\bmod (z) \rightarrow \infty$ and $\bmod (z) \rightarrow 0$.

We see from eq (73), and also from eqs (56) and (57), that the $\mathrm{H}$-functions contain basically two series representations, one in $q_{n}$ and the other in $Q_{r^{\prime}}$ and a remainder term which is nonvanishing when there are higher order poles in the integrand. The terms containing $q_{n}$ are actually those which give the particular solution of eqs (25) and (26). The terms containing $\mathrm{Q}_{r}$ are the solution of the homogeneous equation because, when substituted in eqs (25) and (26), they lead to the homogeneous matrix equation (36) (unless $q_{n}=Q_{r}$ for some $n$ and $r$ ).

Thus, although eqs (56) and (57) are particular solutions of the Hilbert's equation in the q representation, they may still contain a homogeneous part which separates out when integrated over q. We shall, however, refer to these terms also as part of the particular solution to distinguish it from the homogeneous part which has to be separately introduced in order to satisfy eq (12). This part will be discussed a little later in section 3D.

3A. Behavior of $H_{p}(z)$ for large values of $\bmod (z)$

We shall now discuss the behavior of $\mathrm{H}(z)$ given by eq (73) for large mod( $z)$. In this limit, as given by eq (C.22), only the poles in the UHP will contribute to the integral. The corresponding allowed values of $n$ and $Q_{r}$ are then given by eq (c.22). For a moment if we ignore any higher order poles, we find from eqs (73) and (C.22), that $\underline{H}(z)$ will consist of only negative powers of $z$. This shows that the stress will vanish as $\bmod (z) \rightarrow \infty$.

The first series in eq (73) contains negative integral powers, whereas the second series, in general, will contain 
negative fractional powers of $z$. Let us write $Q_{r}$ in terms of real and imaginary parts as

$$
Q_{r}=g_{r}+\iota k_{r^{\prime}}
$$

with the constraint

$$
\mathrm{k}_{r}=\operatorname{Im}\left(Q_{r}\right)>0
$$

The second series in eq (74) can then be written in the form

$$
\mathrm{H}_{\mathrm{p} 2}(z)=\sum_{r} \underline{\mathrm{V}}_{\mathrm{p} 2}\left(\mathrm{Q}_{\mathrm{r}}\right)[\mathrm{z}]^{-\left(\mathrm{k}_{\mathrm{r}}+0.5\right)} \exp \left[\lg _{r} \ln (z)\right]
$$

where

$$
[\text { for } \bmod (z) \rightarrow \infty] \text {, }
$$

$$
\underline{\mathrm{V}}_{\mathrm{p} 2}\left(Q_{\mathrm{r}}, \varepsilon\right)=\underline{\underline{\Psi}}\left(Q_{\mathrm{r}}\right) \xi\left(\iota Q_{\mathrm{r}}\right)
$$

The subscripts $p 2$ on the vectors $\underline{V}$ and $\underline{H}$ identify them as belonging to the second series of the particular solution. In eqs (80) and (81), we have put $\varepsilon=0$ consistent with our hypothesis that the particular value of $Q_{r}$ is not a higher order pole.

We notice from eq (81) that each term falls off with increasing $\bmod (z)$. The exponential factor for each term will be nonunity whenever $g_{r}$, the real part of $Q_{r}$ corresponding to that term is nonzero.

This discussion is valid for those terms which arise from only simple poles. However, as shown below, at least one pole, that at $n=0$, is a second-order pole. For this pole, therefore, we have to evaluate the proper limit at $\varepsilon=0$.

It can be verified that $D(q)$ is zero at $q=q_{0}$ where $q_{0}$ has 
been defined in eq (C.25). For this purpose, we note from eqs $(38)-(41),(84)$ and (85), and by using the definition of various parameters as given in section 2 and Appendix $I-A$, that at $q=q_{0}$, the matrix elements of $M(q)$ and $\zeta(q)$ reduce to

$$
\begin{gathered}
\underline{M}_{A A}\left(q_{0}\right)=2 \operatorname{Re}\left[\underline{\Gamma}^{A}+\underline{\Gamma}^{A} \underline{Q}_{S}^{I}\right], \\
\underline{M}_{A B}\left(q_{0}\right)=-2 \operatorname{Re}\left[\underline{\Gamma}^{A} \underline{Q}_{S}^{I I I}\right], \\
﹎{B A}\left(q_{0}\right)=-2 \operatorname{Re}\left[\bar{\Gamma}^{B} \underline{\Omega}_{S}^{I I}\right], \\
\underline{M}_{B B}\left(q_{0}\right)=-2 \operatorname{Re}\left[\underline{\Gamma}^{B}-\underline{\Gamma}^{B} \underline{Q}_{S}^{I V}\right], \\
\zeta_{A}(0)=M_{A A}\left(q_{0}\right), \\
\zeta_{B}(0)=-\underline{M}_{B A}\left(q_{0}\right) .
\end{gathered}
$$

The quantities $Q_{S}^{I}-Q_{S}^{I V}$ in eqs $(82)-(85)$ have been defined in eqs (A.7)-(A.10) in Appendix I-A. They should not be confused with $Q_{r}$ which denotes a particular value of $q$.

Using eq (A.25) of II, we find that

$$
\underline{M}_{A A}\left(q_{0}\right)-M_{A B}\left(q_{0}\right)=M_{B A}\left(q_{0}\right)-M_{B B}\left(q_{0}\right)=0,
$$

which shows that

$$
D\left(q_{0}\right)=0
$$

Since $E(q)$ also has a zero at $q_{0}$ [see eq (c.17)], we see that the integrand in eq (54) has a second-order pole at $q_{0}=Q_{0}=\iota / 2$. 
The contribution of this second order pole has been evaluated in Appendix I-C. Since this pole is in UHP, it does not contribute to the integral in the limit $\bmod (z) \rightarrow 0$. For large values of $\bmod (z)$, we calculate its contribution by using eq (C.29) as described below.

By comparing eqs (C.16) and (54), we note that in the calculation of $\underline{H}^{A}(z)$, the function $\underline{P}(q)$ as defined by eq (55) for $\mathrm{q}=\mathrm{q}_{0}$ is given by

$$
\underline{p}^{A}\left(q_{0}\right)=\left[\underline{c}_{A A}\left(q_{0}\right) \underline{N}_{A}\left(q_{0}\right)+\underline{c}_{A B}\left(q_{0}\right) \underline{N}_{B}\left(q_{0}\right)\right]
$$

In order to achieve some simplification, it would be useful at this point to derive certain relations between the block elements of the matrix $\underline{c}(q)$ at $q=q_{0}$. From eqs (49) and $(86)-(87)$, we see that

$$
\underline{C}_{A A}\left(q_{0}\right) \zeta_{A}(0)-\underline{C}_{A B}\left(q_{0}\right) \zeta_{B}(0)=D\left(q_{0}\right)=0
$$

and

$$
\underline{c}_{B A}\left(q_{0}\right) \zeta_{A}(0)-\underline{c}_{B B}\left(q_{0}\right) \zeta_{B}(0)=0
$$

We can derive the following relations by taking the product of $\underline{M}(q)$ and $\underline{C}(q)$ in that order and by using eqs (49) and (88):

$$
\underline{c}_{A A}\left(q_{0}\right)+\underline{c}_{B A}\left(q_{0}\right)=0
$$

and

$$
\underline{c}_{A B}\left(q_{0}\right)+\underline{c}_{B B}\left(q_{0}\right)=0
$$

Now we come back to eq (90). From the definition of $\underline{N}(q)$ as given by eqs (42) and (43), we note that

$$
\mathrm{N}_{\mathrm{A}}\left(\mathrm{q}_{0}\right)=-\zeta_{\mathrm{A}}(0)
$$


and

$$
\underline{N}_{B}\left(q_{0}\right)=\zeta_{B}(0)
$$

From eqs (91), (95) and (96) we find that eq (90) gives

$$
\underline{\mathrm{p}}^{\mathrm{A}}\left(\mathrm{q}_{0}\right)=0
$$

Similarly, we can show that

$$
\underline{\mathrm{P}}^{\mathrm{B}}\left(\mathrm{q}_{0}\right)=0
$$

Hence we need to keep only the first term on the RHS of eq (C.29). A similar argument applies to the calculation of $\underline{H}^{B}(z)$. Thus, in the $\operatorname{limit} \varepsilon=0$, we get the result [for $\bmod (z) \rightarrow \infty]$ :

$$
\underline{R}_{H}^{A}(z)=\frac{\iota z^{-1}}{D^{\prime}\left(q_{0}\right)} P^{A^{\prime}}\left(q_{0}\right)
$$

and

$$
\underline{R}_{H}^{B}(z)=\frac{\iota z^{-1}}{D^{\prime}\left(q_{0}\right)} P^{B^{\prime}}\left(q_{0}\right),
$$

where the primes denote differentiation with respect to the argument.

Equations (99) and (100) give the contribution of the second-order pole at $q_{0}$ to the $H$ functions, if there are no other higher order poles in the integrand of the H-functions. In case some other values of $q_{n}$ and $Q_{r}$ coincide, we need to take the limit $\varepsilon=0$ of those terms in a similar manner, and they would be included in $R_{H}(z)$. In those terms in eqs (56) and (57), in which $q_{n}$ is not equal to $Q_{r} \varepsilon$ can be put equal to zero immediately.

The pole at $q_{0}$ as discussed above is effectively a first order pole because $\underline{P}\left(q_{0}\right)$ is also zero along with $D\left(q_{0}\right)$ at $q=q_{0}$. 
This is the reason why logarithmic terms cancel out in the final result given by eqs (99) and (100).

In deriving eqs (99) and (100), we have only considered the degeneracy of the poles between set I and set II that is between $q_{n}$ and $Q_{r}$ as defined by eqs (C.17) and (C.18). It is obvious from eq (C.17) that there cannot be a degeneracy among the poles of set I because no two $q_{n}{ }^{\prime} s$ can be equal.

The poles of set II as defined by eq. (C.18) might be degenerate among themselves. Since the matrix $\underline{M}(q)$ is $6 \times 6$, it would have six eigenvalues. The determinantal equation (C.18) can, therefore, have up to 6 -fold degenerate roots. If $\eta$ is the degeneracy of a particular root, that is if $\eta$ number of $Q_{r}$ are equal, then, as given by the cauchy integral theorem, their contribution to the integral'will contain a factor of the type $[\ln (z)]^{\eta-1}$. The function $\underline{H}(z)$ will not diverge at $\infty$ because the logarithmic term will be multiplied by a negative power of $z$.

3B. Behavior of $H_{p}(z)$ for low values of $\bmod (z)$

In this subsection we shall discuss the form of the particular solution for $\underline{H}(z)$ in the limit $\bmod (z) \rightarrow 0$. The behavior of $\underline{H}(z)$ for low values of $\bmod (z)$ can be analyzed following the same steps as in the preceding subsection for large $\bmod (z)$. The singularities near $\bmod (z) \approx 0$ in the total $\underline{H}(z)$, including the homogeneous solution, will be discussed separately in section $3 \mathrm{E}$.

The expression given in eq (73) for $\underline{H}(z)$ is also valid in the limit $\bmod (z) \rightarrow 0$, but the allowed values of $n$ and $Q_{r}$ will be those given by eq (C.23). The first series in eq (73) will contain only positive integral powers of $z$. These terms will vanish in the limit $\bmod (z) \rightarrow 0$. The second series may contain also fractional powers. Except for those values of $Q_{r}$ which have an imaginary part 
greater than -0.5 , the powers of $z$ in the second series will also be positive. This shows that, apart from the exception given above, the terms in both the series in eq (73) will approach 0 as $\bmod (z)$ goes to 0 .

In the second series, we again write $Q_{r}$ in terms of its real and imaginary parts as given below [c.f. eq (78)]

$$
Q_{r}=g_{r p}-\iota k_{r p}
$$

with the constraint

$$
k_{r p}=-\operatorname{Im}\left(Q_{r}\right)>0
$$

The subscript $p$ on $g_{r}$ and $k_{r}$ is introduced to identify this term as coming from the particular solution. The resulting expression for $\mathrm{H}_{\mathrm{p} 2}(\mathrm{z})$, which is analogous to that given by eq $(80)$ is

$$
H_{p 2}(z)=\sum_{r} \underline{v}_{p 2}\left(Q_{r}\right)[z]^{\left(k_{r p}-0.5\right)} \exp \left[\iota g_{r p} \ln (z)\right]
$$

for $\bmod (z) \rightarrow 0$.

Now we consider the contribution of the higher order poles following the same procedure as used in the preceding subsection. It may be verified that $D(q)$ is zero at $q=Q_{-1}=-l / 2$. Since $E(q)$ is also zero at $q=q_{-1}=-\iota / 2$, the integrand has at least one second-order pole at $q_{-1}=Q_{-1}=-\iota / 2$. Unlike the case for $\bmod (z)$ $\rightarrow \infty$ as discussed in the previous section, $P\left(q_{-1}\right)$ is not zero in this case. The contribution of this second-order pole which is represented by the remainder term, has been evaluated in Appendix I-C [see eq (C.30)] and is given below: 


$$
\begin{aligned}
\underline{R}_{H}(z)=\frac{l}{D^{\prime}\left(q_{-1}\right)} & {\left[\underline{P}^{\prime}\left(q_{-1}\right)+L \underline{P}\left(q_{-1}\right) \operatorname{Ln}(z)\right.} \\
& \left.-0.5 \underline{P}\left(q_{-1}\right) D^{\prime \prime}\left(q_{-1}\right) / D^{\prime}\left(q_{-1}\right)+\pi \underline{P}\left(q_{-1}\right)\right] .
\end{aligned}
$$

Equation (104) shows the $\ln (z)$ behavior of $H(z)$ at low values of $\bmod (z)$. In deriving this equation, we have assumed that the root $Q_{-1}$ of the determinantal equation (C.18) is not degenerate. As remarked earlier, if eq (C.18) has a $\eta$-fold degenerate root at ${ }^{2} r \eta^{\prime} H(z)$ will have terms of the type,

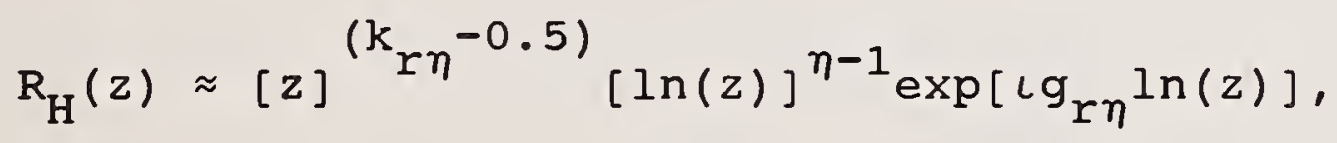

where $g_{r \eta}$ and $k_{r \eta}$ are, respectively, the real and imaginary parts of $Q_{r \eta} \cdot$ If $k_{r \eta}$ is half-integer and $g_{r \eta}=0$, then $E(q)$ will also be 0 at $q=Q_{r \eta}$. This is similar to the case previously considered for which $Q_{r \eta}=-\iota / 2$. This will lead to an additional power of $\ln (z)$ as in eq (104).

3C. Contribution of the homogeneous solution

We now consider whether the homogeneous solution $\mathrm{H}_{0}(z)$ has to be included in the total solution or not. For this purpose we need to calculate the integral of the force functions with the objective of ensuring that eq (12) is satisfied. The integrai required for calculating the force function from the $\mathrm{H}$ function has been evaluated in Appendix I-C. However, we shall not need the explicit expressions for the force functions for calculating the Green's functions; a knowledge of the H-functions will suffice. In this section, therefore, we need only to consider the space integral of the force function, which also has been evaluated in Appendix I-C by using contour integration. 
As shown in Appendix I-C, the only poles which contribute to the force integral are those at $Q_{r}$, which are the zeroes of $D(q)$ and which satisfy the constraint given by eq (C.35). Each value of $Q_{r}$ corresponds to a force term containing $\iota_{r}-0.5$ as the power of $y$ and a stress term containing the same power of $y$. Each of these terms will be the homogeneous solution because, by definition, $D\left(Q_{r}\right)$ will be 0 for all of them. We, therefore, choose additional force terms such that their integral is negative of that given by eq (C.34) so that the total integral of the force function is 0 as required by eq (12). The corresponding H-functions which denote the homogeneous solution are written in the form,

$$
\begin{gathered}
\underline{H}_{0}^{A}(z)=-\sum_{Q_{r} \neq l / 2} v_{0}^{A}\left(Q_{r}\right) z^{l Q_{r}}-0.5, \\
\underline{H}_{0}^{B}(z)=-\sum_{Q_{r}^{\neq l / 2}} v_{0}^{B}\left(Q_{r}\right) z^{l Q_{r}}-0.5, \\
\underline{v}_{0}^{A}\left(Q_{r}\right)=2 \pi \iota \frac{\underline{P}^{A}\left(Q_{r}\right)}{E\left(Q_{r}\right) D^{\prime}\left(Q_{r}\right)},
\end{gathered}
$$

and

$$
\underline{v}_{0}^{B}\left(Q_{r}\right)=2 \pi \frac{\exp \left(\pi Q_{r}\right) \underline{P}^{B}\left(Q_{r}\right)}{E\left(Q_{r}\right) D^{\prime}\left(Q_{r}\right)},
$$

with the constraint

$$
0 \leq \operatorname{Im}\left(Q_{r}\right) \leq 0.5
$$

3D. Singularities in $H(z)$ at $\bmod (z)=0$

Now we discuss the singularities in $\underline{H}(z)$ as $\bmod (z)$ approaches 
0 . As given by eq (110), H(z) contains two main terms, one is the particular solution which is an integral over $q$, and the other is the homogeneous solution which is a sum over $Q_{r}$, where $Q_{r}$ is a root of eq (51) with the constraint given by eq (110).

First, we consider the homogeneous solution. As described in the preceding section, this term will arise only if eq (51) has a solution $Q_{\text {ro }}$ which satisfies the constraint given by eq (109). The additional subscript 0 has been introduced to identify this term as arising from the homogeneous solution. This singularity can be expressed as

$$
H(z) \approx(z)^{-\left(k_{r 0}+0.5\right)} \exp \left[\lg _{r 0} \ln (z)\right],
$$

where the weight (coefficient) of each singular term is given by eqs (108) and (109), and $g_{r o}$ and $k_{r o}$ are, respectively, the real and imaginary parts of $Q_{\text {ro' }}$

$$
Q_{r 0}=g_{r 0}+\iota k_{r 0}
$$

where

$$
0 \leq k_{r 0} \leq 0.5
$$

We see from eq (111) that the exponent of the singularity varies between 0 and -1 . In addition, if the real part of $Q_{r 0}$ (in addition to the constraint on its imaginary part) is nonzero, $\underline{H}(z)$ will also have a logarithmic--cos or $\sin \left[g_{r_{p}} \ln (z)\right]--t y p e$ oscillatory component, which is represented by the exponential factor in eq (111). This oscillatory component is of the same type which arises in the stress and the displacement field near an interfacial crack in bimaterial composites (see, for example, II). Depending upon the material parameters of the two constituents of the composite, this term can be significant. The singular term as 
given by eq (111) will of course be zero if a $Q_{r}$ does not exist which satisfies the constraint given by eq (113).

Next, let us identify the singular terms in the particular solution for $\underline{H}(z)$, which is given by an integral over $q$ in eq (54). As shown in section $3 A$, for $\bmod (z) \approx 0$, this integral can be represented in terms of two series and a remainder term as given in eq (73). As explained in section 3B, the first series in eq (73) has no singularity. The second term in eq (73) is a series over $Q_{r^{\prime}}$ where $Q_{r}$ are solutions of eq (51) with the constraint that $\operatorname{Im}\left(Q_{r}\right)$ is negative since these terms arise from the poles in the LHP. This series is given by eq (103).

We see from eq (103) that only those terms will be singular at $\bmod (z)=0$ for which $k_{r p}<0.5$. If the real part of $Q_{r}$ is nonzero, the logarithmic oscillatory factor will also be present. Thus we obtain the following condition for a term in the second series in eq (73) to be singular:

$$
0 \leq k_{r p} \leq 0.5
$$

The corresponding singular terms in $\underline{H}(z)$ are of the type,

$$
\mathrm{H}_{\mathrm{p} 2}(z) \approx(z)^{\left(\mathrm{k}_{\mathrm{rp}}-0.5\right)} \exp \left[\lg _{\mathrm{rp}} \ln (z)\right]
$$

where $k_{r p}$ has to satisfy the constraint given by eq (114), and the additional subscript 2 in $H$ identifies the second series in eq (73) as the origin of this singularity. The exponent of this singularity varies between 0 and -0.5 .

Finally, we consider the singularities in the remainder term $R_{H}(z)$ in eq (73), which arise if there are higher order poles. The contribution of one second-order pole at $q=-\iota / 2$, at which both $E(q)$ and $D(q)$ are 0 , is given by eq (104). This gives a 
singularity in $\underline{\mathrm{H}}(\mathrm{z})$ which varies as $\ln (z)$. This singular term has no oscillatory factor since the real part of $Q_{-1}$ is zero. If eq (51) has degenerate roots, $\underline{H}(z)$ will have higher order logarithmic singularities as given by eq (105). In general, the logarithmic oscillatory factor will also be present as shown in eq (105).

The singularities in the $\mathrm{H}$-function arise from the homogeneous part of eq (36) and are, in general, independent of the inhomogeneity, that is the RHS in eq (36).

\section{Expressions for the Green's Functions}

In this section we shall give the final expressions for the displacement Green's function and the stress-2 Green's function. The former gives the displacement and the latter the stress components $\tau_{i 2}$ for a unit line force. The stress-1 Green's function, which gives the stress components $\tau_{i l}$ for a unit line force is given by eqs (15) and (16). We shall give the results only for the case when the unit line force is in the UHP at the point $\left(\mathrm{x}^{\prime}, \mathrm{Y}^{\prime}\right)$ with $\rho_{\alpha}$ defined as

$$
\rho_{\alpha}=x^{\prime}+p_{\alpha} y^{\prime}
$$

The corresponding expressions for the case when the unit line force is in the LHP can be obtained by a simple change of variables, $y^{\prime}$ and $\rho$.

4A. Displacement Green's Function

$$
\begin{aligned}
\underline{G}^{A}\left(\underline{x}, \underline{x}^{\prime}\right) & =-\frac{1}{\pi} \operatorname{Re} \sum_{\alpha} \underline{\gamma}^{A}(\alpha) \ln \left(z_{\alpha}^{A}-\rho_{\alpha}^{A}\right) \phi \\
& -\frac{1}{\pi} \operatorname{Re} \sum_{\alpha \beta} \underline{\gamma}^{A}(\alpha) \underline{Q}_{\beta}^{I} \ln \left(z_{\alpha}^{A}-\rho_{\beta}^{A}\right) \phi
\end{aligned}
$$




$$
\begin{aligned}
& +\iota \sum_{\alpha}\left[\underline{\gamma}^{A}(\alpha) \underline{U}^{A}\left(\frac{z_{\alpha}^{A}+\varepsilon}{p_{\alpha}^{A}}\right)+\underline{\gamma}^{A}(\alpha) \underline{U}^{A}\left(\frac{\bar{z}_{\alpha}^{-A}+\varepsilon}{p_{\alpha}^{A}}\right)\right] \\
& +\iota \sum_{\alpha \beta}\left[\underline{E}_{A A}(\alpha, \beta) \underline{U}^{A}\left(\frac{z_{\alpha}^{A}}{p_{\beta}^{A}}\right)+\underline{E}_{A A}(\alpha, \beta) \underline{U}^{A}\left(\frac{\bar{z}_{\alpha}^{A}}{p_{\beta}^{A}}\right)\right] \\
& -\iota \sum_{\alpha \beta}\left[\underline{E}_{A B}(\alpha, \beta) \underline{U}^{B}\left(\frac{z_{\alpha}^{A}}{p_{\beta}^{B}}\right)+\bar{E}_{A B}(\alpha, \beta) \underline{U}^{B}\left(\frac{\bar{z}_{\alpha}^{A}}{\bar{p}_{\beta}^{B}}\right)\right]
\end{aligned}
$$

(for $\left.0 \leq x_{2} \leq \infty\right)$

and

$$
\begin{aligned}
& \underline{G}^{B}\left(\underline{x}, \underline{x}^{\prime}\right)=-\frac{1}{\pi} \operatorname{Re} \sum_{\alpha \beta} \underline{\gamma}^{-B}(\alpha) \underline{Q}_{\beta}^{I I} \ln \left(\bar{z}_{\alpha}^{-B} \bar{\rho}_{\beta}^{-A}\right) \phi \\
& +\iota \sum_{\alpha \beta}\left[\underline{E}_{\mathrm{BA}}(\alpha, \beta) \underline{U}^{\mathrm{A}}\left(\frac{\bar{z}_{\alpha}^{-\mathrm{A}}}{\bar{p}_{\beta}^{\mathrm{A}}}\right)+\bar{E}_{\mathrm{BA}}(\alpha, \beta) \underline{U}^{\mathrm{A}}\left(\frac{\mathrm{z}_{\alpha}^{\mathrm{B}}}{\mathrm{p}_{\beta}^{\mathrm{A}}}\right)\right] \\
& +\iota \sum_{\alpha}\left[\underline{\gamma}^{B}(\alpha) \underline{U}^{B}\left(\frac{z_{\alpha}^{B} \varepsilon}{p_{\alpha}^{B}}\right)+\underline{\gamma}^{B}(\alpha) \underline{U}^{B}\left(\frac{\bar{z}_{\alpha}^{B} \varepsilon}{\bar{p}_{\alpha}^{B}}\right)\right] \\
& -\iota \sum_{\alpha \beta}\left[\underline{E}_{B B}(\alpha, \beta) \underline{U}^{B}\left(\frac{\bar{z}_{\alpha}^{B}}{p_{\beta}^{B}}\right)+\bar{E}_{B B}(\alpha, \beta) \underline{U}^{B}\left(\frac{z_{\alpha}^{B}}{\bar{p}_{\beta}^{B}}\right)\right] \\
& \text { (for } \left.-\infty \leq x_{2} \leq 0\right) \text {, }
\end{aligned}
$$

where

$$
\underline{E}_{A A}(\alpha, \beta)=\underline{\gamma}^{A}(\alpha) \underline{Q}_{\beta}^{I},
$$




$$
\begin{gathered}
\underline{E}_{\mathrm{AB}}(\alpha, \beta)=\underline{\gamma}^{\mathrm{A}}(\alpha) \underline{\mathrm{Q}}_{\beta}^{\mathrm{III}}, \\
\underline{\mathrm{E}}_{\mathrm{BA}}(\alpha, \beta)=\underline{\underline{\gamma}}^{\mathrm{B}}(\alpha) \underline{\mathrm{Q}}_{\beta}^{\mathrm{II}}, \\
\underline{\mathrm{E}}_{\mathrm{BB}}(\alpha, \beta)=\underline{\bar{\gamma}}^{\mathrm{B}}(\alpha) \underline{\mathrm{Q}}_{\beta}^{\mathrm{IV}}, \\
\underline{\mathrm{U}}^{\mathrm{A}}(\mathrm{z})=-\frac{1}{2 \pi \iota} \int_{0}^{\infty} \mathrm{F}^{\mathrm{A}}(t) \ln (z-t) \mathrm{dt} \\
=\int \mathrm{H}^{\mathrm{A}}(z) \mathrm{dz}
\end{gathered}
$$

and

$$
\begin{aligned}
\underline{U}^{B}(z)= & -\frac{1}{2 \pi l} \int_{-\infty}^{0} F^{B}(t) \ln (z-t) d t \\
& =\int H^{B}(z) d z .
\end{aligned}
$$

In deriving eqs (117) and (118) from eqs (10) and (11), we have neglected terms which contain factors of the type $\ln \left(p_{\alpha}\right)$ if they represent only rigid body displacements. The integrals in eqs (123) and (124) are indefinite integrals. These integrals can be evaluated by using either the integral or the series representation for the H-functions which have been derived in the previous sections. It can be verified by using eq (6) that eqs (117) and (118) yield eqs (15) and (16) for stress components $\tau_{i 1}$.

4B Stress-2 Green's Function

The stress-2 Green's function which can be derived from eqs (117) and (118) by following the procedure given in section 2 or I 
and II, are given below:

$$
\begin{aligned}
\underline{T}_{2}\left(\underline{x}, \underline{x}^{\prime}\right) & =-\frac{1}{\pi} \operatorname{Re} \sum_{\alpha} \underline{\sigma}^{A}(\alpha)\left[z_{\alpha}^{A}-\rho_{\alpha}^{A}\right]^{-1} \\
& -\frac{1}{\pi} \operatorname{Re} \sum_{\alpha \beta} \underline{\sigma}^{A}(\alpha) \underline{Q}_{\beta}^{I}\left[z_{\alpha}^{A}-\bar{\rho}_{\beta}^{A}\right]^{-1} \\
+ & \iota \sum_{\alpha}\left[\left\{\underline{\sigma}^{A}(\alpha) / \underline{p}_{\alpha}^{A}\right\} \underline{H}^{A}\left(\frac{z_{\alpha}^{A}+\varepsilon}{p_{\alpha}^{A}}\right)+\left\{\underline{\sigma}^{A}(\alpha) / \bar{p}_{\alpha}^{A}\right\} \underline{H}^{A}\left(\frac{z_{\alpha}^{-A} \varepsilon}{\bar{p}_{\alpha}^{A}}\right)\right] \\
+ & \iota \sum_{\alpha \beta}\left[\underline{D}_{A A}(\alpha, \beta) \underline{H}^{A}\left(\frac{z_{\alpha}^{A}}{\bar{p}_{\beta}^{A}}\right)+\underline{D}_{A A}(\alpha, \beta) \underline{H}^{A}\left(\frac{\bar{z}_{\alpha}^{A}}{p_{\beta}^{A}}\right)\right] \\
& -\iota \sum_{\alpha \beta}\left[\underline{D}_{A B}(\alpha, \beta) \underline{H}^{B}\left(\frac{z_{\alpha}^{A}}{p_{\beta}^{B}}\right)+\underline{D}_{A B}(\alpha, \beta) \underline{H}^{B}\left(\frac{\bar{z}_{\alpha}^{A}}{\bar{p}_{\beta}^{B}}\right]\right]
\end{aligned}
$$

and

$$
\begin{aligned}
& \underline{T}_{2}^{B}\left(\underline{x}, \underline{x}^{\prime}\right)=-\frac{1}{\pi} \operatorname{Re} \sum_{\alpha \beta} \underline{\sigma}^{B}(\alpha) \underline{Q}_{\beta}^{I I}\left[\bar{z}_{\alpha}^{B}-\bar{\rho}_{\beta}^{A}\right]^{-1} \\
& +\iota \sum_{\alpha \beta}\left[\underline{\mathrm{D}}_{\mathrm{BA}}(\alpha, \beta) \underline{\mathrm{H}}^{\mathrm{A}}\left(\frac{\overline{\mathrm{z}}_{\alpha}^{\mathrm{B}}}{\overline{\mathrm{p}}_{\beta}^{\mathrm{A}}}\right)+\overline{\mathrm{D}}_{\mathrm{BA}}(\alpha, \beta) \underline{\mathrm{H}}^{\mathrm{A}}\left(\frac{\mathrm{z}_{\alpha}^{\mathrm{B}}}{\mathrm{p}_{\beta}^{\mathrm{A}}}\right)\right] \\
& +\iota \sum_{\alpha}\left[\left\{\underline{\sigma}^{\mathrm{B}}(\alpha) / \mathrm{p}_{\alpha}^{\mathrm{B}}\right\} \underline{H}^{\mathrm{B}}\left(\frac{\mathrm{z}_{\alpha}^{\mathrm{B}}+\varepsilon}{\mathrm{p}_{\alpha}^{\mathrm{B}}}\right)+\left\{\bar{\sigma}^{\mathrm{B}}(\alpha) / \overline{\mathrm{p}}_{\alpha}^{\mathrm{B}}\right\} \underline{H}^{\mathrm{B}}\left(\frac{\bar{z}_{\alpha}^{\mathrm{B}}+\varepsilon}{\bar{p}_{\alpha}^{\mathrm{B}}}\right)\right]
\end{aligned}
$$




$$
-\iota \sum_{\alpha \beta}\left[\underline{\mathrm{D}}_{\mathrm{BB}}(\alpha, \beta) \underline{H}^{\mathrm{B}}\left(\frac{\bar{z}_{\alpha}^{\mathrm{B}}}{\mathrm{p}_{\beta}^{\mathrm{B}}}\right)+\underline{\mathrm{D}}_{\mathrm{BB}}(\alpha, \beta) \underline{H}^{\mathrm{B}}\left(\frac{\mathrm{z}_{\alpha}^{\mathrm{B}}}{\overline{\mathrm{p}}_{\beta}^{\mathrm{B}}}\right)\right],
$$

where

$$
\begin{aligned}
& \underline{\mathrm{D}}_{\mathrm{AA}}(\alpha, \beta)=\underline{\sigma}^{\mathrm{A}}(\alpha) \mathrm{Q}_{\beta}^{I} / \bar{p}_{\beta}^{\mathrm{A}}, \\
& \underline{\mathrm{D}}_{\mathrm{AB}}(\alpha, \beta)=\underline{\sigma}^{\mathrm{A}}(\alpha) \underline{\mathrm{Q}}_{\beta}^{\mathrm{III}} / \mathrm{p}_{\beta}^{\mathrm{B}}, \\
& \underline{\mathrm{D}}_{\mathrm{BA}}(\alpha, \beta)=\bar{\sigma}^{\mathrm{B}}(\alpha) \underline{\mathrm{Q}}_{\beta}^{\mathrm{II}} / \overline{\mathrm{p}}_{\beta}^{\mathrm{A}},
\end{aligned}
$$

and

$$
\underline{\mathrm{D}}_{\mathrm{BB}}(\alpha, \beta)=\underline{\bar{\sigma}}^{\mathrm{B}}(\alpha) \underline{\mathrm{e}}_{\beta}^{\mathrm{IV}} / \mathrm{p}_{\beta}^{\mathrm{B}}
$$

In the next two sections, we shall illustrate the formalism developed in the preceding sections by applying it to two simple cases: the antiplane-strain problem and the plane strain problem in a cubic solid containing a $\Sigma-5$ tilt grain boundary. 


\section{Application to the Antiplane-Strain Problem}

For illustration, we apply the formalism developed in this paper to the simple case of antiplane-strain in a bimaterial composite containing a free surface. This case is of little practical interest but has the important advantage of simplicity. It can, therefore, serve the purpose of illustrating the mathematical technique for calculating the SI Green's function without unnecessarily clouding the essential features of the technique. The experience gained in treating this simple case should be useful to readers in solving a realistic problem. The calculation of the SI Green's function for a more realistic case has been given in the next section.

In the antiplane-strain case, all the $3 \times 3$ matrices such as $\underline{\gamma}$, $\underline{\sigma}, \underline{\Omega}$ and of course the Green's function itself reduce to $1 \times 1$ matrices, that is, pure numbers. Further, in this case the indices $\alpha, \beta$, etc., will take only one value, that is, $\alpha=\beta=1$. We assume the solids $A$ and $B$ which constitute our composite model to be cubic. This is the same model which has been considered in $I$ and II. Values of various parameters for this model are given below. Some of these have been calculated in I and II; others can be obtained quite simply by following the same method.

$$
\begin{gathered}
\mathrm{p}_{\alpha}=\mathrm{p}_{\beta}=\iota, \\
\gamma^{\mathrm{A}, \mathrm{B}}(\alpha)=\gamma_{\mathrm{S}}^{\mathrm{A}, \mathrm{B}}=1 / 2 \mathrm{~d}_{\mathrm{A}, \mathrm{B}}, \\
\sigma^{\mathrm{A}, \mathrm{B}}(\alpha)=\sigma_{\mathrm{S}}^{\mathrm{A}, \mathrm{B}}=\iota / 2, \\
\mathrm{Q}_{\alpha}^{I}=\mathrm{Q}_{\mathrm{S}}^{I}=\mathrm{Q}_{\alpha}^{I V}=\mathrm{Q}_{\mathrm{S}}^{I V}=\mathrm{d}, \\
\mathrm{Q}_{\alpha}^{I I}=\mathrm{Q}_{\mathrm{S}}^{I I}=2 \mathrm{~d}_{\mathrm{B}}, \\
\mathrm{Q}_{\alpha}^{I I I}=\mathrm{Q}_{\mathrm{S}}^{I I I}=-2 \mathrm{~d}_{\mathrm{A}}, \\
\Omega^{\mathrm{A}, \mathrm{B}}=1 / 2,
\end{gathered}
$$




$$
\begin{aligned}
& \mathrm{K}_{\mathrm{AA}}(\alpha, \beta)=\iota d / 2 ; \quad \mathrm{K}_{\mathrm{AB}}(\alpha, \beta)=\iota d_{A} \text {, } \\
& \mathrm{K}_{\mathrm{BA}}(\alpha, \beta)=\mathrm{R}(\alpha, \beta)=\iota \mathrm{d}_{\mathrm{B}} \text {, } \\
& \mathrm{K}_{\mathrm{BB}}(\alpha, \beta)=\mathrm{P}(\alpha, \beta)=-0.5 \text { ८d, } \\
& \mathrm{J}^{\mathrm{A}, \mathrm{B}}=\Gamma=-\iota / 2, \\
& \mathrm{E}_{\mathrm{AA}}=\mathrm{d} / 2 \mathrm{~d}_{\mathrm{A}} ; \mathrm{E}_{\mathrm{AB}}=-1 \text {, } \\
& E_{B A}=1 ; E_{B B}=d / 2 d_{B} \text {, } \\
& a_{\alpha \beta}=-1=\exp (\pi \iota) ; e_{\alpha \beta}=-1=\exp (-\pi \iota), \\
& b_{\alpha \beta}=d_{\alpha \beta}=1 \text {, } \\
& \mathrm{d}=\mathrm{d}_{\mathrm{A}}-\mathrm{d}_{\mathrm{B}} \text {, } \\
& d_{A, B}=c_{44}^{A, B} /\left[c_{44}^{A}+c_{44}^{B}\right] \text {, } \\
& d_{A}+d_{B}=1 \text {. }
\end{aligned}
$$

Substituting the values of the constants in eqs (131)-(141) into eqs (117) and (118), we obtain the following expressions for the displacement Green's function for a unit line force applied at $\underline{x}^{\prime}$ in the UHP :

i. $\underline{x}$ in UHP

$$
\begin{aligned}
G\left(x, x^{\prime}\right) & =\left(-1 / 2 \pi d_{A}\right) \operatorname{Re}[\ln (z-\rho)+d \ln (z-\rho)] \\
& +\frac{\iota}{2 d_{A}}\left[U^{A}\left(\frac{z+\varepsilon}{\iota}\right)+U^{A}\left(\frac{\bar{z}+\varepsilon}{-\iota}\right)\right]+\frac{\iota d}{2 d_{A}}\left[U^{A}\left(-\frac{z}{\iota}\right)+U^{A}\left(\frac{\bar{z}}{\iota}\right)\right] \\
+ & \iota\left[U^{B}\left(\frac{z}{\iota}\right)+U^{B}\left(-\frac{\bar{z}}{\iota}\right)\right],
\end{aligned}
$$


ii. $\underline{x}$ in $\underline{L H P}$

$$
\begin{aligned}
G^{B}\left(x, x^{\prime}\right) & =(-1 / \pi) \operatorname{Re}[\ln (\bar{z}-\bar{\rho})]+\iota\left[U^{A}\left(-\frac{\bar{z}}{\iota}\right)+U^{A}\left(\frac{z}{\iota}\right)\right] \\
+ & \frac{\iota}{2 d_{B}}\left[U^{B}\left(\frac{z+\varepsilon}{\iota}\right)+U^{B}\left(\frac{\bar{z}+\varepsilon}{-\iota}\right)\right] \\
& -\frac{\iota d}{2 d_{B}}\left[U^{B}\left(\frac{\bar{z}}{\iota}\right)+U^{A}\left(-\frac{z}{\iota}\right)\right] .
\end{aligned}
$$

Following the procedure given in section 2, or directly from eggs (15) and (16), we obtain the following expressions for the stress $\left(\tau_{i 1}\right)$ Green's functions:

$$
\begin{aligned}
\mathrm{T}_{1}^{\mathrm{A}}\left(\mathrm{x}, \mathrm{x}^{\prime}\right)= & -\frac{1}{2 \pi} \operatorname{Re}\left[\frac{1}{(\mathrm{z}-\rho)}+\frac{\mathrm{d}}{(\mathrm{z}-\bar{\rho})}\right] \\
& +\frac{1}{2}\left[\mathrm{H}^{\mathrm{A}}[(\mathrm{z}+\varepsilon) / \iota]-\mathrm{H}^{\mathrm{A}}[-(\bar{z}+\varepsilon) / \iota]\right. \\
- & \frac{d}{2}\left[\mathrm{H}^{\mathrm{A}}[-\mathrm{z} / \iota]-\mathrm{H}^{\mathrm{A}}[\bar{z} / \iota]\right]+\mathrm{d}_{\mathrm{A}}\left[\mathrm{H}^{\mathrm{B}}[\mathrm{z} / \iota]-\mathrm{H}^{\mathrm{B}}[-\bar{z} / \iota]\right]
\end{aligned}
$$

and

$$
\begin{aligned}
T_{1}^{B}\left(x, x^{\prime}\right)= & -\frac{d_{B}}{\pi} \operatorname{Re}\left[\frac{1}{(\bar{z}-\bar{\rho})}\right]-d_{B}\left[H^{A}[-\bar{z} / \iota]-H^{A}[z / \iota]\right] \\
& -\frac{1}{2}\left[H^{B}[-(\bar{z}+\varepsilon) / \iota]-H^{B}[(z+\varepsilon) / \iota]\right. \\
& -\frac{d}{2}\left[H^{B}[\bar{z} / \iota]-H^{B}[-z / \iota]\right],
\end{aligned}
$$

44 
where, in view of eqs (131) and (A.5),

$$
z=x_{1}+\iota x_{2} \equiv x+\iota y
$$

As in section 2, we obtain the following equations for the two H-functions by using the boundary condition given by eqs (4) and (5) or directly from eqs (25) and (26):

$$
\begin{aligned}
{\left[H^{A}(y+\iota \varepsilon)-H^{A}(y-\iota \varepsilon)\right]=} & -\frac{1}{2 \pi \iota}\left\{\frac{1}{y+\iota \rho}-\frac{1}{y-\iota \bar{\rho}}\right. \\
& \left.+d\left[\frac{1}{y+\iota \bar{\rho}}-\frac{1}{y-\iota \rho}\right]\right\}
\end{aligned}
$$

and

$$
\left[H^{B}(y+\iota \varepsilon)-H^{B}(y-\iota \varepsilon)\right]=\frac{d^{B}}{\pi \iota}\left[\frac{1}{y-\iota \bar{\rho}}-\frac{1}{y+\iota \rho}\right] .
$$

Following the method given in Appendix $I-B$ and the steps given in section 2, we obtain the following solution of eqs (146) and (147).

$$
\begin{gathered}
v^{A}(q)=s(-\iota \rho)-s(\iota \bar{\rho})+d[s(-\iota \bar{\rho})-s(\iota \rho)], \\
v^{B}(q)=2 d^{B} \iota \exp (\pi q)[s(-\iota \bar{\rho})-s(\iota \rho)], \\
H_{p}^{A}(z)=\int_{-\infty}^{\infty} v^{A}(q) z^{\iota q-0.5} d q,
\end{gathered}
$$

and 


$$
H_{p}^{B}(z)=\int_{-\infty}^{\infty} v^{B}(q) z^{l q-0.5} d q
$$

where

$$
s(p)=\frac{1}{2 \pi}\left[\exp (-2 \pi q) / E^{2}(q)\right] \rho^{-\iota q-0.5}
$$

Now we consider evaluation of the integral in eq (150) with $V^{A}(q)$ given by eq (148). The integrand has second-order poles at all values of $q_{n}$ [see eq (c.17) for definition of $q_{n}$ ]. We can use eq (C.24) for evaluating this integral by putting all $Q_{r}=q_{n}$ and accounting for the second-order poles as described in Appendix $I-C$. This would give a series in $q_{n}$ which can be easily summed. We can also evaluate this integral directly by making the substitution $t=\exp (-2 \pi q)$. The limits of integration over $t$ become 0 to $\infty$. The integral can then be carried out by using the same contour as used in Appendix I-C for evaluating $I(z)$ in eq (c.1). The integral for $\mathrm{H}^{\mathrm{B}}(\mathrm{z})$ in eq (151) can be evaluated in a similar manner. Thus we obtain the result

$$
\begin{aligned}
\mathrm{H}_{\mathrm{p}}^{\mathrm{A}}(z)= & -\frac{1}{2 \pi \iota}[\mathrm{S}(z,-\iota \rho)-S(z, \iota \bar{\rho}) \\
& +\mathrm{d}(\mathrm{S}(z,-\iota \bar{\rho})-S(z, \iota \rho))]
\end{aligned}
$$

and

$$
H_{p}^{B}(z)=-\frac{d^{B}}{\pi \iota}[s(z, \iota \bar{\rho})-s(z,-\iota \rho)],
$$

where

$$
S(z, \rho)=\frac{\iota}{2 \pi(z-\rho)} \ln (z / \rho)
$$


In eqs (153) and (154), the subscript $p$ on the H-functions indicates that they are particular solutions of the Hilbert's equation. We have to add the homogeneous solution to the particular solution unless the corresponding force functions satisfy the condition given by eq (12). It is therefore necessary, as discussed in section 2 , to evaluate the integral of the force functions which is given below.

In the present case, in view of the Plemelj relation, as given by eq (B.42), the force functions are directly given by eqs (146) and (147). The integral of the force functions can now be easily carried out. The result is

$$
I_{F}=\int_{0}^{Y_{\infty}} F^{A}(y) d y+\int_{-y_{\infty}}^{0} F^{B}(y) d y=1,
$$

where we have to take the limit $\mathrm{y}_{\infty}=\infty$.

In deriving eq (156), we have accounted for the fact that in the definition of $\mathrm{H}^{\mathrm{A}}(\mathrm{z})$ in eq (146), the cut is on the positive real axis. Hence, for the discontinuity in $H^{A}(y)$ at the real axis, we have used the relations

$$
\begin{gathered}
\iota=\exp (\pi \iota / 2),-\iota=\exp (3 \pi \iota / 2) ; \\
\ln \left(y_{\infty}+\iota \rho\right)=\ln y_{\infty}, \ln \left(y_{\infty}-\iota \rho\right)=\ln y_{\infty}+2 \pi \iota ;
\end{gathered}
$$

where $\rho$ has a positive real part and $\bmod \left(\rho / Y_{\infty}\right) \ll 1$.

Similarly, for $\mathrm{H}^{\mathrm{B}}(\mathrm{z})$ in eq (146), the cut is on the negative side of the real axis. In this case, therefore,

$$
\iota=\exp (\pi \iota / 2),-\iota=\exp (-\pi \iota / 2) ;
$$

and 
$\ln \left(-y_{\infty}+\iota \rho\right)=\ln y_{\infty}+\pi \iota, \ln \left(-y_{\infty}-\iota \rho\right)=\ln y_{\infty}-\pi \iota ;$

We find from eq (158) that the force functions given by eqs (148) and (149) do not satisfy the constraint given by eq (12). We therefore add the following force functions, which are solutions of the homogeneous Hilbert equation, that is, eqs (143) and (144) with their RHS equal to zero.

$$
F_{0}^{A}(y)=-\varepsilon / \pi\left(y^{2}+\varepsilon^{2}\right)
$$

and

$$
F_{0}^{B}(y)=-\varepsilon / \pi\left(y^{2}+\varepsilon^{2}\right)
$$

where, in both eqs (161) and (162), the limit $\varepsilon=+0$ has to be taken. The two force functions have a delta function dependence on $y$. In the limit $\varepsilon=0$, they approach zero for all values of $y$ except for $y=0$. Their integral is finite, and they make a nonvanishing contribution to the H-functions. The corresponding H-functions which represent the homogeneous solution of the Hilbert equation, are given below.

and

$$
H_{0}^{A}(z)=\frac{1}{2 \pi \iota} z^{-1}
$$

$$
H_{0}^{B}(z)=\frac{1}{2 \pi \iota} z^{-1}
$$

The integrals of the force functions as given by eqs (161) and (162) can be evaluated as follows

$$
I_{F O}=\operatorname{Lim}_{y_{\infty}=\infty}\left[\int_{0}^{y_{\infty}} F_{0}^{A}(y) d y+\int_{-y_{\infty}}^{0} F_{0}^{B}(y) d y\right] .
$$


Using eqs (157) -- (160), and taking the limit $\varepsilon=+0$, and $\mathrm{y}_{\infty}=$ $\infty$, we obtain

$$
I_{F O}=-1
$$

In writing the force functions given by eqs (161) and (162) the total integral has been divided equally between $F^{A}$ and $F^{B}$ but this division is quite arbitrary. Since both the force functions are applied at the same point, that is, $y=0$, what matters is their total resultant. Thus, in general, the coefficient in eq (161) could be taken to be any number--say $\xi$ instead of 0.5 , provided the coefficient in eq (162) is taken to be $1-\xi$.

From eqs (156) and (166) we see that if the force functions given by eqs (161) and (162) are added to those given by eqs (143) and (144), the constraint given by eq (12) will be satisfied. These forces will contribute zero stress at the free surface, since they are solution of the homogeneous equation. Thus the final solution which will satisfy eq (12) and all the other boundary conditions is given by

$$
\mathrm{H}^{\mathrm{A}, \mathrm{B}}(\mathrm{z})=\mathrm{H}_{\mathrm{p}}^{\mathrm{A}, \mathrm{B}}(\mathrm{z})+\mathrm{H}_{0}^{\mathrm{A}, \mathrm{B}}(\mathrm{z}),
$$

where the particular solutions $H_{p}^{A, B}(z)$ are given by eqs (153) and (154) and the homogeneous solutions $H_{0}^{A, B}(z)$ by eqs (163) and (164). Although the two expressions given by eqs (163) and (164) look similar, the former has to be evaluated by taking a cut on the positive half of the real axis and the latter by taking the cut on the negative half of the real axis.

The stress-1 Green's functions are thus given by eq (144) in the UHP and eq (145) in the LHP with the H-functions given by eq (167). The displacement Green's functions are given by eqs (142) and (143) in terms of the U-functions which, according to eqs 
(123) and (124), are obtained by integrating the $\mathrm{H}$ functions. The result is

$$
\begin{aligned}
U^{A}(z)=-\frac{1}{2 \pi \iota} & {[v(z,-\iota \rho)-v(z, \iota \bar{\rho})} \\
+ & d(v(z,-\iota \bar{\rho})-v(z, \iota \rho))]+\frac{1}{2 \pi \iota} \ln (z)
\end{aligned}
$$

and

$$
U^{B}(z)=-\frac{d^{B}}{\pi \iota}[v(z, \iota \bar{\rho})-v(z,-\iota \rho)]+\frac{1}{2 \pi \iota} \ln (z)
$$

where

$$
\begin{aligned}
v(z, \rho)=\frac{\iota}{2 \pi} & {\left[-0.5(\ln z)^{2}+\ln z \ln (z-\rho)-\ln \rho \ln (z-\rho)\right.} \\
& \left.-\sum_{n} \frac{1}{n^{2}}(\rho / z)^{n}\right] \quad(\text { for }|z / \rho|>1)
\end{aligned}
$$

and

$$
\begin{aligned}
\mathrm{v}(\mathrm{z}, \rho)=\frac{\mathrm{l}}{2 \pi} & {[-\ln \rho \ln \mathrm{z}+\ln \mathrm{z} \ln (\rho-\mathrm{z})-\ln \rho \ln (\rho-\mathrm{z})} \\
& \left.+\sum_{\mathrm{n}} \frac{1}{\mathrm{n}^{2}}(\mathrm{z} / \rho)^{\mathrm{n}}\right] \quad(\text { for }|\mathrm{z} / \rho|<1),
\end{aligned}
$$

and where the sum is over all positive integers $\dot{n}(n=1,2, \ldots)$. The last logarithmic term in eqs (170) and (171) corresponds to the homogeneous solution.

The stress-2 Green's functions can be obtained from the displacement Green's functions by using the method given in section 2 or directly from eqs (125) and (126). These are given below. 
i. $x$ in UHP :

$$
\begin{aligned}
\mathrm{T}_{2}^{\mathrm{A}}\left(\mathrm{x}, \mathrm{x}^{\prime}\right)= & -\frac{\iota}{2 \pi} \operatorname{Re}\left[\frac{1}{(z-\rho)}+\frac{d}{(z-\bar{\rho})}\right] \\
& +\frac{\iota}{2}\left[\mathrm{H}^{\mathrm{A}}[(\mathrm{z}+\varepsilon) / \iota]+\mathrm{H}^{\mathrm{A}}[-(\bar{z}+\varepsilon) / \iota]\right] \\
& -\frac{\iota d}{2}\left[\mathrm{H}^{\mathrm{A}}[-\mathrm{z} / \iota]+\mathrm{H}^{\mathrm{A}}[\bar{z} / \iota]\right] \\
& +\iota \mathrm{d}_{A}\left[\mathrm{H}^{\mathrm{B}}[z / \iota]+\mathrm{H}^{\mathrm{B}}[-\bar{z} / \iota]\right]
\end{aligned}
$$

ii. $\underline{x}$ in LHP :

$$
\begin{aligned}
\mathrm{T}_{2}^{\mathrm{B}}\left(\mathrm{x}, \mathrm{x}^{\prime}\right)= & -\frac{\iota \mathrm{d}_{\mathrm{B}}}{\pi} \operatorname{Re}\left[\frac{1}{(\bar{z}-\bar{\rho})}\right]+\iota \mathrm{d}_{\mathrm{B}}\left[\mathrm{H}^{\mathrm{A}}[-\bar{z} / \iota]+\mathrm{H}^{\mathrm{A}}[\mathrm{z} / \iota]\right] \\
& +\frac{\iota}{2}\left[\mathrm{H}^{\mathrm{B}}[-(\bar{z}+\varepsilon) / \iota]+\mathrm{H}^{\mathrm{B}}[(\mathrm{z}+\varepsilon) / \iota]\right. \\
& +\frac{\iota \mathrm{d}}{2}\left[\mathrm{H}^{\mathrm{B}}[\bar{z} / \iota]-\mathrm{H}^{\mathrm{B}}[-\mathrm{z} / \iota]\right]
\end{aligned}
$$

The calculated values of the displacement field $u(\underline{x})$, stress-1 component $\tau_{31}(\underline{x})$ and the stress-2 component $\tau_{32}(\underline{x})$ have been shown as 3-D plots in figure I-2 through $I-4$ respectively. In these calculations $d_{A}$ and $d_{B}$ have been taken to 0.8 and 0.2 respectively. The unit line force has been applied at $\underline{x}^{\prime}=(3$, 1.5). The vector $\underline{x}^{\prime}$ is chosen so that it does not fall at any point of the mesh where the Green's function is calculated. This is to avoid the characteristic singularity in the Green's function at $\underline{x}=\underline{x}^{\prime}$. We see from figure $I-2$ through $I-4$ that $\tau_{31}$ is 0 at $x_{1}=0$ and $u(\underline{x})$ and $\tau_{32}$ are continuous at $x_{2}=0$ whereas $\tau_{31}$ is discontinuous at $x_{2}=0$. 
An important point concerning the numerical calculations: In deriving the expression for $\mathrm{H}^{\mathrm{A}}(\mathrm{z})$, we have taken the cut on the positive half of the real axis. This implies that the argument of the variable changes by $2 \pi \iota$ when it crosses the real axis on the positive side. This has to be properly accounted for in the computer programming. For example the complex log should have the following values in the limit $\varepsilon=+0$ where $y$ is real and positive.

$$
\ln (y+\iota \varepsilon)=\ln y ; \ln (y-\iota \varepsilon)=\ln y+2 \pi \iota
$$

and

$$
\ln (-y+\iota \varepsilon)=\ln y+\pi \iota ; \ln (-y-\iota \varepsilon)=\ln y+\pi \iota \text {. }
$$

On the other hand, in normal Fortran on most computers, the branch values are taken as

$$
\ln (y+\iota \varepsilon)=\ln y, \ln (y-\iota \varepsilon)=\ln y ;
$$

and

$$
\ln (-y+\iota \varepsilon)=\ln y+\pi \iota, \ln (-y-\iota \varepsilon)=\ln y-\pi \iota .
$$

In the derivation of $\mathrm{H}^{\mathrm{B}}(\mathrm{z})$, the cut has been taken on the negative side of the real axis. In this case the argument of the variable would change from $\pi \iota$ to $-\pi \iota$ as it goes across the cut. The branch values of the log function for this cut will be the same as given by eqs (176) and (177).

In the numerical calculations for the $H$ functions, it is therefore necessary to program the computer so that the appropriate branch values are taken, that is, as given by eqs (174) and (175) for the $H^{A}$-function and by eqs (176) and (177) for the $\mathrm{H}^{\mathrm{B}}$-function. 


\section{Application to a Cubic Crystal Containing a $\Sigma-5$ Grain Boundary --Plane-Strain Problem}

As a further illustration of the formulation given in section 3, we apply it to calculating the SI Green's function for a cubic crystal containing a $\Sigma-5$ tilt boundary. We have chosen this particular case for illustration because of the current interest in the structure of grain boundaries and because its high symmetry simplifies the calculations considerably. The SI Green's function as calculated in this section can be applied to a variety of problems concerning the elastic properties of the grain boundaries such as dislocation pile-up and crack propagation.

A tilt boundary in a solid can be visualized as a composite solid in which two half solids are welded together along the boundary and the crystallographic axes of the solids are oriented with respect to each other. In particular, the $\Sigma-5$ boundary in a cubic solid can be modelled as follows (see, for example, [24-25]). Consider two pieces of the same cubic material with their crystallographic axes parallel to the coordinate axes. Rotate one of the pieces about the $\mathrm{z}$-axis by an angle $\theta=$ $\tan ^{-1}(3 / 5)$ while keeping the other piece fixed. Weld the two pieces together so that they form a planar interface. This interface will be the $\Sigma-5$ grain boundary.

The cubic solid, which we have chosen for our calculations, is stainless steel. The Green's functions for this solid without the free surface have been calculated in I. In this paper we use the same model as described in $I$. We label the solid which has its crystallographic axes parallel to the coordinate axes as the solid $A$, that is, in the UHP. The solid, which has been rotated as described in the preceding paragraph, is labelled as the solid B, that is, in the LHP. 
The values [18] of the elastic constants of stainless steel are taken as follows (in units of $\mathrm{c}_{44}$ ): $\mathrm{c}_{11}=2.2, \mathrm{c}_{12}=1.3, \mathrm{c}_{44}$ $=1.0$. These will be the elastic constants of the solid $A$ in our model. The elastic constants of the solid B, that is, the rotated solid, can be obtained by using the rotation transformation law for the fourth-rank tensors as given below:

$$
c_{i j k l}^{B}=s_{i i^{\prime}}, s_{j j^{\prime}} s_{k k^{\prime}} s_{l l^{\prime}} c_{i^{\prime} j^{\prime} k^{\prime} l^{\prime}}^{A},
$$

where $\underline{S}$ is the matrix of rotation. Its elements are

and

$$
\mathrm{S}_{11}=\mathrm{S}_{22}=\cos \theta
$$

$$
\mathrm{s}_{12}=-\mathrm{s}_{21}=\sin \theta
$$

where, for a $\Sigma-5$ boundary, $\theta=\tan ^{-1}(3 / 5)$.

The calculation of various parameters for the present model has been described in I. Here we only give the values of those parameters which are needed for the present calculation. As given in $I$, in the present case, $\alpha$ and $\beta$ which label the roots of eq (A.19), take only the values 1 and 2. The third root, $\alpha=3$, corresponds to the antiplane-strain mode which has already been discussed in the preceding section.

The most basic parameters required for the Green's function calculation are $p_{\alpha}^{A, B}$, which are the roots of eq (A.19), and the matrices $\underline{\gamma}(\alpha)$ and $\underline{\sigma}(\alpha)$ as defined by eqs (A.17) and (A.15) respectively. The expressions for these parameters are given below. Other matrices can be easily calculated in terms of these matrices by using various relations given in section 2 . In the expressions given below the superscripts $A$ and $B$ are not shown for notational brevity. 


$$
\begin{aligned}
& p_{1}=\left[\left(1-k^{2}\right) / 2\right]^{1 / 2}+\iota\left[\left(1+k^{2}\right) / 2\right]^{1 / 2}, \\
& p_{2}=-\left[\left(1-k^{2}\right) / 2\right]^{1 / 2}+\iota\left[\left(1+K^{2}\right) / 2\right]^{1 / 2}, \\
& \gamma_{11}(\alpha)=z_{\alpha}\left(1+\zeta p_{\alpha}^{2}\right), \\
& \gamma_{12}(\alpha)=\gamma_{21}(\alpha)=-z_{\alpha} \beta_{0} p_{\alpha} \\
& \gamma_{22}(\alpha)=z_{\alpha}\left(\zeta+p_{\alpha}^{2}\right), \\
& \sigma_{11}(\alpha)=z_{\alpha} p_{\alpha}\left(1-\beta_{0}+\zeta p_{\alpha}^{2}\right), \\
& \sigma_{12}(\alpha)=\mathrm{z}_{\alpha}\left[\left(1-\beta_{0}\right) \mathrm{p}_{\alpha}^{2}+\zeta\right], \\
& \sigma_{21}(\alpha)=z_{\alpha}\left(-1+\beta_{0}-\zeta p_{\alpha}^{2}\right), \\
& \sigma_{22}(\alpha)=c_{44} z_{\alpha} p_{\alpha}\left(\zeta^{2}-\beta_{0}^{2}+\beta_{0}+\zeta p_{\alpha}^{2}\right),
\end{aligned}
$$

where $\alpha=1$ or 2 , and

$$
\begin{aligned}
& \mathrm{z}_{\alpha}=-\frac{(-1)^{\alpha}}{4 \mathrm{c}_{11} \mathrm{p}_{\alpha}}\left[1-\mathrm{K}^{4}\right]^{-1 / 2}, \\
& \mathrm{~K}^{2}=1+\left(\delta_{0} / \zeta\right)\left(\beta_{0}+0.5 \delta_{0}\right), \\
& \zeta=\mathrm{c}_{11} / \mathrm{c}_{44}, \\
& \beta_{0}=\left(\mathrm{c}_{12}+\mathrm{c}_{44}\right) / \mathrm{c}_{44}, \\
& \text { and } \\
& \delta_{0}=\left(\mathrm{c}_{11}-\mathrm{c}_{12}-2 \mathrm{c}_{44}\right) / \mathrm{c}_{44} .
\end{aligned}
$$

The point of application of the unit force is taken to be the

55 
same as in the previous section, that is, at $\underline{x}^{\prime}=(3 \cdot 0,1.5)$. As mentioned in the previous section, for numerical convenience, the value of the vector $\underline{x}^{\prime}$ should be chosen so that it does not fall at any point of the mesh where the Green's function is calculated. This is to avoid the characteristic singularity in the Green's function at $x=x^{\prime}$.

The calculated values of the the displacement $u_{1}(\underline{x})$ and the stress components $\tau_{11}(\underline{x}), \tau_{21}(\underline{x})=\tau_{12}(\underline{x})$, and $\tau_{22}(\underline{x})$ have been shown as 3D plots in figures I-5 through $I-8$ respectively. The plot for $u_{2}(\underline{x})$ is similar to that of $u_{1}(\underline{x})$ and, therefore, has not been given.

We see from figure I-5, I-7, and I-8 that $u_{1}(\underline{x}), \tau_{12}(\underline{x})$ and $\tau_{22}(\underline{x})$ are continuous across the interface at $x_{2}=0$ as required by the boundary conditions given by eqs (2) and (3). The stress component $\tau_{11}(\underline{x})$ is not continuous across the interface as shown in figure $I-6$. The components $\tau_{21}(\underline{x})$ and $\tau_{11}(\underline{x})$ are zero at the free surface, that is, at $x_{1}=0$ as required by the boundary condition given by eq (4) and (5).

The singularities in the stresses will be discussed in the following paper on the generalized plane-strain problem. As mentioned in section $3 D$, the singularities in $H(z)$ arise from the homogeneous part of eq (36) and, in general, do not depend upon the RHS of eq (36). As we shall see in the following paper, the matrix $M(q)$ is exactly the same in the present plane-strain case and the generalized plane-strain case.

It may be emphasized again that, in using the formulation of this paper for numerical calculations, the values of the various functions such as $\ln (z)$ and $z^{l q}$ over the upper and lower branches of the cut have to evaluated carefully according to the scheme given by eqs $(174)-(177)$. 


\section{Summary of Main Results}

In this paper we have obtained the displacement Green's functions and the stress Green's functions for a general anisotropic bimaterial composite solid which has a plane interface and a free surface normal to the interface. These are applicable to a variety of plane-strain problems and also account for displacements and forces normal to the plane.

We find that the stress field is singular near the intersection of the free surface and the interface. The singularities are of the type $r^{-\eta}$ and $[\ln (r)]^{n}$ where $\eta$ is a positive number between 0 and 1 and $n$ is a positive integer between 0 and 6 . The actual values of $\eta$ and $n$ and the coefficient, i.e., the weight of the singularities, depend upon the material properties of the solids. In addition to the singularity, an oscillatory behavior of cos or $\sin [\ln (r)]$ type, may also be present in certain cases.

A method of solution of the generalized inhomogeneous vector Hilbert problem has been given. The method is simple and convenient for applications to different problems in the stress analysis of materials involving cracks and interfaces in which the inhomogeneity and the nonsingular part of the Hilbert's kernel are of the form $1 /(y-\rho)$ where $y$ is a real variable and $\rho$ is complex. For this purpose, we have introduced a complex transform which is of the form $y^{\iota q-0.5}$ where $\iota=V(-1)$ and $q$ is a real variable. This transform seems to be particularly interesting, because it is an eigenfunction of the Hilbert's kernel and is orthogonal over both variables $y$ and $q$ in the domain $0 \leq y \leq \infty$ and $-\infty \leq q \leq \infty$. 


\section{Appendix I-A}

\section{Elastic Green's Function for an Infinite Bimaterial Composite}

In this appendix we quote the expressions for the elastic Green's function for a bimaterial composite as obtained in I and used for calculation of Green's function for a cracked solid in II. The expressions given below have been taken from II and are in a form which is more convenient for present application. The coordinate system used for these expressions is the same as shown in figure $I-1$. The indices $\alpha$ and $\beta$ take the values 1,2 , or 3 .

The Green's function given below is the displacement Green's function $G\left(\underline{x}, \underline{x}^{\prime}\right)$, which gives the displacement field at the point $\underline{x}$ when a unit line force is applied at the point $\underline{x}^{\prime}$. As in I and II, we distinguish between four different cases corresponding to $\underline{x}$ and $\underline{x}^{\prime}$ being in UHP and LHP.

1. $\underline{x}$ and $\underline{x}^{\prime}$ both in $\operatorname{UHP}\left(\mathrm{x}_{2} \geq 0 ; \mathrm{x}_{2}^{\prime} \geq 0\right)$ :

$$
\begin{aligned}
\mathrm{G}_{0}^{\mathrm{AA}}\left(\underline{x}, \underline{x}^{\prime}\right)= & -\frac{1}{\pi} \sum_{\alpha} \underline{\gamma}^{\mathrm{A}}(\alpha) \ln \left(z_{\alpha}^{\mathrm{A}}-\rho_{\alpha}^{\mathrm{A}}\right) \\
& -\frac{1}{\pi} \sum_{\alpha \beta} \underline{\gamma}^{\mathrm{A}}(\alpha) \underline{\mathrm{Q}}_{\beta}^{I} \ln \left(z_{\alpha}^{\mathrm{A}}-\bar{\rho}_{\beta}^{\mathrm{A}}\right),
\end{aligned}
$$

2. $\underline{x}$ in LHP, $\underline{x}^{\prime}$ in $\operatorname{UHP}\left(x_{2} \leq 0 ; x_{2}^{\prime} \geq 0\right)$ :

$$
G_{0}^{B A}\left(\underline{x}, \underline{x}^{\prime}\right)=-\frac{1}{\pi} \sum_{\alpha \beta} \underline{\bar{z}}^{-B}(\alpha) \underline{\mathrm{Q}}_{\beta}^{I I} \ln \left(\bar{z}_{\alpha}^{\mathrm{B}}-\bar{\rho}_{\beta}^{\mathrm{A}}\right),
$$


3. $\underline{x}$ in UHP, $\underline{x}^{\prime}$ in $\operatorname{LHP}\left(x_{2} \geq 0 ; x_{2}^{\prime} \leq 0\right)$ :

$\mathrm{G}_{0}^{\mathrm{AB}}\left(\underline{x}, \underline{x}^{\prime}\right)=\frac{1}{\pi} \sum_{\alpha \beta} \underline{\underline{\gamma}}^{\mathrm{A}}(\alpha) \underline{\underline{Q}}_{\beta}^{\mathrm{III}} \ln \left(z_{\alpha}^{\mathrm{A}} \alpha_{\beta}^{\mathrm{A}}\right)$,

4. $\underline{x}$ and $\underline{x}^{\prime}$ both in $\operatorname{LHP}\left(\mathrm{x}_{2} \leq 0 ; \mathrm{x}_{2}^{\prime} \geq 0\right)$ :

$$
\begin{aligned}
G_{0}^{\mathrm{BB}}\left(\underline{x}, \underline{x}^{\prime}\right)= & -\frac{1}{\pi} \sum_{\alpha} \underline{\underline{\gamma}}^{-\mathrm{B}}(\alpha) \ln \left(\bar{z}_{\alpha}^{\mathrm{B}}-\bar{\rho}_{\alpha}^{\mathrm{B}}\right) \\
& +\frac{1}{\pi} \sum_{\alpha \beta} \underline{\bar{\gamma}}^{\mathrm{B}}(\alpha) \underline{\Omega}_{\beta}^{\mathrm{IV}} \ln \left(\bar{z}_{\alpha}^{\mathrm{B}}-\rho_{\beta}^{\mathrm{B}}\right),
\end{aligned}
$$

where

$$
\begin{aligned}
& z_{\alpha}^{A, B}=x_{1}+p_{\alpha}^{A, B} x_{2}, \\
& \rho_{\alpha}^{A, B}=x_{1}^{\prime}+p_{\alpha}^{A, B} x_{2}^{\prime}, \\
& \underline{Q}_{\alpha}^{I}=\underline{M}\left[\underline{\sigma}^{A}(\alpha)-\underline{\sigma}_{s}^{B}\left(\underline{\bar{\gamma}}_{s}^{B}\right)^{-1} \underline{\gamma}^{A}(\alpha)\right], \\
& \underline{Q}_{\alpha}^{I I}=\underline{N}\left[\underline{\sigma}^{A}(\alpha)-\underline{\sigma}_{s}^{A}\left(\underline{\gamma}_{s}^{A}\right)^{-1} \underline{\underline{\gamma}}^{A}(\alpha)\right],
\end{aligned}
$$

$\underline{\mathrm{g}}_{\alpha}^{\text {III }}=\underline{\mathrm{M}}\left[\underline{\sigma}^{\mathrm{B}}(\alpha)-\underline{\bar{\sigma}}_{\mathrm{S}}^{\mathrm{B}}\left(\underline{\bar{\gamma}}_{\mathrm{s}}^{\mathrm{B}}\right)^{-1} \underline{\underline{\gamma}}^{\mathrm{B}}(\alpha)\right]$,

$\underline{Q}_{\alpha}^{I V}=\underline{N}\left[\underline{\sigma}^{B}(\alpha)-\underline{\sigma}_{S}^{A}\left(\underline{\gamma}_{s}^{A}\right)^{-1} \underline{\gamma}^{B}(\alpha)\right]$,

$\underline{M}=\left(\underline{\gamma}_{S}^{A}\right)^{-1}\left[\bar{\sigma}_{S}^{B}\left(\underline{\bar{\gamma}}_{S}^{B}\right)^{-1}-\underline{\sigma}_{S}^{A}\left(\underline{\gamma}_{S}^{A}\right)^{-1}\right]^{-1}$,

and 
$\underline{N}=\left(\underline{\underline{\gamma}}_{s}^{-B}\right)^{-1}\left[\underline{\sigma}_{s}^{B}\left(\underline{\bar{\gamma}}_{s}^{-B}\right)^{-1}-\underline{\sigma}_{s}^{A}\left(\underline{\gamma}_{s}^{A}\right)^{-1}\right]^{-1}$

(Note. The following formulas are valid for either superscript, A or B, corresponding to the solid in UHP or LHP, respectively.)

$$
\begin{gathered}
\underline{\gamma}_{s}=\sum_{\alpha} \underline{\gamma}(\alpha), \\
\underline{\sigma}_{s}=\sum_{\alpha} \underline{\sigma}(\alpha), \\
\underline{\sigma}(\alpha)=\underline{L}^{I I}(\alpha) \underline{\gamma}(\alpha), \\
\gamma_{i j}(\alpha)=\frac{\iota}{\operatorname{aq}_{1}^{4}} \frac{L_{i j}^{I I}(\alpha)=c_{i 2 k 1}+p_{\alpha} c_{i 2 k 2},}{\left(p_{\alpha}-\bar{p}_{\alpha}\right) \prod_{\alpha \neq \beta}\left[\left(p_{\alpha}-p_{\beta}\right)\left(p_{\alpha}-\bar{p}_{\beta}\right)\right],} \\
\text { (for } \left.q_{2}=q_{1} p_{\alpha}\right),
\end{gathered}
$$

$\Gamma_{i j}^{\Lambda}(g)$ is the ij cofactor of the Christoffel matrix $\Lambda$ defined by

$$
\Lambda_{i j}(q)=c_{i k j l} q_{k} q_{l}
$$

$q_{1}$ and $q_{2}$ are components of the wave vector $q$ and $p_{\alpha}$ is obtained such that $q_{2}=p_{\alpha} q_{1}$ is a root of the equation,

$$
|| \Lambda(q)||=0
$$

||$\Lambda||$ denotes the determinant of the matrix $\Lambda$ and $a$ is the coefficient of $q_{2}{ }^{6}$ in ||$\Lambda||$, and

$$
\operatorname{Im}\left(p_{\alpha}\right)>0
$$


In analogy with eqs (A.13) and (A.14), we define the following quantities as sums over $\alpha$ :

$$
\mathrm{Q}_{\mathrm{S}}^{I, I I, I I I, I V}=\sum_{\alpha} \mathrm{Q}_{\alpha}^{I, I I, I I I, I V}
$$

Several useful relations between the various parameters of the Green's function (such as $\underline{\gamma}, \underline{\sigma}, \underline{Q}_{\alpha}^{I}$ ) have been given in Appendix A of II. Two more relations which involve the constants introduced in this paper are

$$
\begin{gathered}
\underline{\Lambda}^{\mathrm{D}} \underline{\gamma}=0, \\
\Omega(\alpha) / p_{\alpha}=-\sigma(\alpha)=J(\alpha),
\end{gathered}
$$

where

$$
\underline{\Lambda}_{i k}^{D}=c_{i 1 k 1}+p_{\alpha}\left(c_{i 1 k 2}+c_{i 2 k 1}\right)+p_{\alpha}^{2} c_{i 2 k 2} .
$$




\section{Appendix I-B}

\section{Solution of Generalized Inhomogeneous Vector Hilbert Problem}

In this appendix we give a solution of the generalized inhomogeneous vector Hilbert problem. The corresponding scalar problem, its properties and solution have been discussed in detail in the excellent treatise by Muskhelishvili [20]. The vector problem has been discussed by Vekua [21]. Our method of solution as given below is different from those given in $[20,21]$ and is based upon the use of a complex transform. This method should be particularly convenient for those types of kernels and inhomogeneities which occur in problems of stress analysis in solids containing surfaces, interfaces, and cracks.

First we consider the scalar problem as defined below:

$$
D H(Y+\iota \varepsilon)+\bar{D} H(Y-\iota \varepsilon)+\sum_{\alpha} c_{\alpha} H\left(a_{\alpha} y\right)=f(y)
$$

where $D, C_{\alpha}$ and $a_{\alpha}$ are complex numbers, $\alpha$ is a summation index which takes an arbitrary but finite range of values, $t$ and $y$ are real variables which vary between 0 and $\infty, \varepsilon$ is a small positive number which approaches zero in the limit, $f(y)$ is a known function, and $\mathrm{H}(\mathrm{z})$ for any complex $\mathrm{z}$, is defined as

$$
H(z)=\frac{1}{2 \pi \iota} \int_{0}^{\infty} \frac{F(t)}{t-z} d t .
$$

The integral in eq (B.2) has to be interpreted as the principal value integral for $z$ on the real axis when the integral is singular. The first two terms in eq (B.1) correspond to the 
principal value of the integral in the definition of the $\mathrm{H}$ function. The function $F(t)$ in eq (B.2) is the unknown function which has to be determined from eq (B.1).

In eq (B.1), $f(y)$ is the inhomogeneity, which is zero for the homogeneous Hilbert problem. If $\mathrm{C}_{\alpha}=0$, this equation will have no nonsingular term in the kernel and is then called the special (not generalized) Hilbert problem (the prefix 'special' is usually omitted). The particular form of the nonsingular kernel as given in eq (B.1) is of the type $1 /(t-a y)$ where $a$ is complex, and is of special interest in problems concerning stress analysis in solids containing surfaces, interfaces, and cracks.

We solve eq (B.1) by using a complex transform method. For this purpose we introduce the following function of two real variables, $q$ and $y(0 \leq y \leq \infty$ and $-\infty \leq q \leq \infty)$ :

$$
\mathrm{T}(\mathrm{q}, \mathrm{y})=\mathrm{y}^{\text {lq-0.5 }}
$$

By using the values of the branch points as given in Appendix I-C, we can verify that $T(q, y)$ is an eigenfunction of the homogeneous Hilbert problem given by the LHS of eq (B.1). Further, the functions $T(q, y)$ are orthogonal in $q$ as well as $y$ space as given below:

$$
\int_{0}^{\infty} T(q, y) \bar{T}\left(q^{\prime}, y\right) d y=2 \pi \delta\left(q-q^{\prime}\right)
$$

and

$$
\int_{-\infty}^{\infty} T(q, y) \bar{T}\left(q, y^{\prime}\right) d q=2 \pi \delta\left(y-y^{\prime}\right)
$$


These orthogonality relations can be verified by using the following standard definition of the delta function:

$$
\delta(y)=\frac{1}{2 \pi} \int_{-\infty}^{\infty} \exp (\llcorner q y) d q
$$

The integral in eq (B.4) can be evaluated by using the substitution $x=$ In $y$. In writing eq (B.5), we have used the following property of the delta function of an arbitrary differentiable function $\mathrm{p}(\mathrm{y})$ of $\mathrm{y}$ :

$$
\delta[p(y)]=\delta\left(y-y_{0}\right)\left[\frac{d p(y)}{d y}\right]_{y=y_{0}}^{-1},
$$

where $y=y_{0}$ is a real root of the equation $p(y)=0$.

We now write $F(t)$ in terms of its transform $v(q)$ as

$$
F(t)=\int_{-\infty}^{\infty} v(q) t^{i q-0.5} d q
$$

Substituting for $F(t)$ from eq (B.8) in eq (B.2), and carrying out the integration over $y$ as shown in Appendix I-C (see eq C.7), we obtain the following result for $\mathrm{H}(\mathrm{z})$ :

$$
H(z)=\int_{-\infty}^{\infty} V(q) z^{i q-0.5} d q
$$

where

$$
V(q)=V(q) / E(q)
$$

and 


$$
E(q)=1 /[1+\exp (-2 \pi q)]
$$

As described in Appendix I-C, in deriving eq (C.7), we have taken a cut on the positive real axis (see figure I-9). The values of argument just above and just below the real axis are therefore taken to be 0 and $2 \pi$, respectively, as given in eqs (C.3) and (C.4). Thus, we get the following, in the limit $\varepsilon=+0$,

$$
H(y+\iota \varepsilon)=\int_{-\infty}^{\infty} V(q) y^{i q-0.5} d q
$$

and

$$
H(y-\iota \varepsilon)=-\int_{-\infty}^{\infty} V(q) \exp (-2 \pi q) y^{i q-0.5} d q
$$

In order to solve eq (B.1), we use the representation of $H(z)$ as given by eq (B.9) and use eqs (B.12) and (B.13) for the first two terms. Then we multiply both sides of eq $(B .1)$ by $\bar{T}(q, y)$, i.e., $y^{-\iota q-0.5}$, and integrate over $y$ from 0 to $\infty$. Using the orthogonality relation as given by eq (B.4), we obtain the result

$$
V(q)=[M(q)]^{-1} N(q)
$$

where

$$
M(q)=\left[D-\bar{D} \exp (-2 \pi q)+\sum c_{\alpha} a_{\alpha}^{l q-0.5}\right]
$$

and

$$
N(q)=\frac{1}{2 \pi} \int_{0}^{\infty} f(y) y^{-\iota q-0.5} d y
$$


In many cases of practical interest such as stress analysis in solids, the function $f(y)$ is of the form

$$
f(y)=\sum_{\alpha} R_{\alpha} /\left[y-\rho_{\alpha}\right]
$$

where $\rho_{\alpha}$ is complex. In such cases, the integral on the RHS of eq (B.16) can be easily obtained by using the same contour as used for deriving eq (C.7) in Appendix I-C. The result, which can be obtained by replacing $q$ by $-q$ (not $\iota$ by $-\iota$ ) in eq (C.7) is given below:

$$
N(q)=\frac{\iota \exp (-2 \pi q)}{E(q)} \sum_{\alpha} R_{\alpha}\left(\rho_{\alpha}\right)^{-\iota q-0.5}
$$

Equation (B.14) along with eqs (B.8), (B.9) and (B.10) gives the particular solution of eq (B.1). In order to obtain the general solution, we have to add a solution of the homogeneous part of eq (B.1) to the particular solution. The homogeneous solution can be written in terms of $Q_{r}$ as

$$
H_{0}(z)=\sum_{r} h\left(Q_{r}\right) z^{\iota Q_{r}-0.5},
$$

where $h\left(Q_{r}\right)$ are arbitrary constants; and $Q_{r}$, which may be complex, is a solution of the following equation:

$$
M\left(Q_{r}\right)=0 \text {. }
$$

The arbitrary constants can be determined with the help of the boundary conditions on $F(t)$.

Now we consider the vector problem which arises if there is more than one unknown function $F(t)$ and, of course, an equal 
number of equations. In this case we regard eq (B.1) as a matrix equation. If there are $n$ unknown functions and $n$ equations, then $\underline{D}$ and $\underline{C}_{\alpha}$ in eq (B.1) will be $n \times n$ square matrices, whereas $\underline{H}(z)$, $\underline{f}(\mathrm{y})$ will be $\mathrm{n} \times 1$ column matrices or $\mathrm{n}$-component vectors.

Similarly, $\underline{M}(q)$ and $\underline{N}(q)$ will be $n \times n$ and $n \times 1$ matrices respectively. Equation (B.20) will become a determinantal equation and $\underline{h}\left(Q_{r}\right)$ will be an eigenvector of $\underline{M}\left(Q_{r}\right)$ corresponding to a zero eigenvalue. One of the components of this eigenvector will remain arbitrary, which has to be determined from the boundary conditions. With these modifications, the solution given above remains valid for the vector Hilbert problem.

As a special case of the general method, we solve the vector Hilbert problem for two unknown functions. This solution is used in this paper (section 2), where the unknown functions are the two force functions $F^{A}$ and $F^{A}$. As in section 2 , we label the unknown functions with $A$ and $B$ and also label the corresponding matrix elements of eq (B.1) by the same indices. Equation (B.1) now becomes a set of two simultaneous equations as given below:

$$
\begin{aligned}
D_{A A} & H^{A}(y+\iota \varepsilon)+\bar{D}_{A A} H^{A}(y-\iota \varepsilon)+\sum_{\alpha} c_{\alpha A A} H^{A}\left(a_{\alpha} y\right) \\
& +\sum_{\alpha} c_{\alpha A B} H^{B}\left(b_{\alpha} y\right)=f_{A}(y) \quad(0 \leq y \leq \infty)
\end{aligned}
$$

and

$$
\begin{aligned}
\sum_{\alpha} C_{\alpha B A} & H^{A}\left(c_{\alpha} y\right)+D_{B B} H^{B}(y+\iota \varepsilon)+\bar{D}_{B B} H^{B}(y-\iota \varepsilon) \\
+ & \sum_{\alpha} c_{\alpha B B} H^{B}\left(d_{\alpha} y\right)=f_{B}(y) \quad(-\infty \leq y \leq 0) .
\end{aligned}
$$


The H-functions are now defined as

$$
H^{A}(z)=\frac{1}{2 \pi \iota} \int_{0}^{\infty} \frac{F^{A}(t)}{t-z} d t
$$

and

$$
H^{B}(z)=\frac{1}{2 \pi \iota} \int_{-\infty}^{0} \frac{F^{B}(t)}{t-z} d t .
$$

As in eq (B.8), the force functions are written in the following form:

$$
F^{A}(t)=\int_{-\infty}^{\infty} v_{A}(q) t^{i q-0.5} d q
$$

and

$$
F^{B}(t)=\int_{-\infty}^{\infty} v_{B}(q)|t|^{i q-0.5} d q
$$

The corresponding expressions for the H-functions are

$$
H^{A}(z)=\int_{-\infty}^{\infty} V_{A}(q) z^{i q-0.5} d q
$$

and

$$
H^{B}(z)=\int_{-\infty}^{\infty} V_{B}(q) z^{i q-0.5} d q
$$

Using eq (B.25), the integration for $\mathrm{H}^{\mathrm{A}}(\mathrm{z})$ in eq (B.23) is carried out as in eq (C.7). The result is identical to that given by eqs (B.9) and (B.10) except that the superscript $A$ has to be added to the relevant matrices. The corresponding integral for $\mathrm{H}^{\mathrm{B}}(\mathrm{z})$ has also been evaluated in Appendix I-C [see eq (C.13)]. 
In case of $\mathrm{H}^{\mathrm{B}}(z)$, we have taken the cut on the negative real axis. The argument of $z$ is taken to be $\pi$ just above the real axis and $-\pi$ just below it. The corresponding values of the H-functions, as obtained by using eqs (C.I1) and (C.12) are given below: (in the limit $\varepsilon=+0 ;-\infty \leq y \leq 0)$ :

$$
H^{B}(y+\iota \varepsilon)=-\iota \int_{-\infty}^{\infty} V_{B}(q) \exp (-\pi q)|y|^{i q-0.5} d q
$$

and

$$
H^{B}(y-\iota \varepsilon)=\iota_{-\infty}^{\infty} V_{B}(q) \exp (\pi q)|y|^{i q-0.5} d q
$$

To solve eqs (B.21) and (B.22), we proceed as before, except that we multiply eq $(B .21)$ by $y^{-\iota q-0.5}$ and eq (B.22) by $|y|^{-\iota q-0.5}$ and then integrate over $y$ between the limits given in eqs (B.21) and (B.22) to ensure the orthogonality of the transform. Thus, we obtain the following particular solution for the force functions and the corresponding H-functions:

$$
\begin{gathered}
v_{A}(q)=v_{A}(q) / E(q)=M_{A A}^{I} N_{A}+M_{A B}^{I} N_{B}, \\
V_{B}(q)=\iota v_{B}(q) \exp (-\pi q) / E(q)=M_{B A}^{I} N_{A}+M_{B B}^{I} N_{B},
\end{gathered}
$$

where $\underline{\mathrm{M}}^{\mathrm{I}}$ is the inverse of $\underline{\mathrm{M}}$. The matrix elements of $\underline{\mathrm{M}}$ and $\underline{N}$ are

$$
\begin{gathered}
\mathrm{M}_{\mathrm{AA}}=\mathrm{D}_{\mathrm{AA}}-\overline{\mathrm{D}}_{\mathrm{AA}} \exp (-2 \pi q)+\sum_{\alpha} \mathrm{c}_{\alpha \mathrm{AA}}\left(\mathrm{a}_{\alpha}\right)^{i q-0.5,} \\
\mathrm{M}_{\mathrm{AB}}=\sum_{\alpha} \mathrm{C}_{\alpha \mathrm{AB}}\left(\mathrm{b}_{\alpha}\right)^{i q-0.5,}
\end{gathered}
$$




$$
\begin{gathered}
M_{B A}=\sum_{\alpha} c_{\alpha B A}\left(-c_{\alpha}\right) i q-0.5, \\
M_{B B}=-\iota\left[D_{B B} \exp (-\pi q)-\bar{D}_{B B} \exp (\pi q)\right]+\sum_{\alpha} c_{\alpha B B}\left(-d_{\alpha}\right) i q-0.5, \\
N_{A}(q)=\frac{1}{2 \pi} \int_{0}^{\infty} f_{A}(y) y^{-\iota q-0.5} d y \quad(0 \leq y \leq \infty),
\end{gathered}
$$

and

$$
N_{B}(q)=\frac{1}{2 \pi} \int_{\infty}^{0} f_{B}(y)|y|^{-\iota q-0.5} d y \quad(-\infty \leq y \leq 0)
$$

Equations (B.31) and (B.32), when substituted into eqs (B.27) and (B.28), give the particular solution for the H-functions. The homogeneous solution is given by eq (B.19), where $\underline{h}_{\left(Q_{r}\right)}$ is now identified as an eigenvector of the matrix $M\left(Q_{r}\right)$ for a zero eigenvalue with $Q_{r}$ as a solution of the following determinantal equation,

$$
\left\|M\left(Q_{r}\right)\right\|=0
$$

The final solution is the sum of the particular solution and the homogeneous solution as given below:

$$
H^{A}(z)=\int_{-\infty}^{\infty} V_{A}(q) z^{i q-0.5} d q+\sum_{r} h_{A}\left(Q_{r}\right) z^{\left\llcorner Q_{r}-0.5\right.}
$$

and

$$
H^{B}(z)=\int_{-\infty}^{\infty} V_{B}(q) z^{i q-0.5} d q+\sum_{Y} h_{B}\left(Q_{r}\right) z^{L Q_{r}-0.5} .
$$


One of the two components of the eigenvector $h_{A}\left(Q_{r}\right)$ and $h_{B}\left(Q_{r}\right)$ is arbitrary and has to be determined from the boundary conditions or the constraints imposed on the force functions.

The force function can be obtained directly from the $\mathrm{H}$-functions by using $\mathrm{Plemelj}$ relation [20]

$$
\underline{F}(y)=\underline{H}(Y+\iota \varepsilon)-\underline{H}(Y-\iota \varepsilon) .
$$

In the present case, eq (B.42) may be easily verified by using the transforms of $\underline{F}$ and $\underline{H}$ as given by eqs (B.31) and (B.32).

In many problems concerning stress analysis in solids, the inhomogeneity $f(y)$ is of the form given by eq (B.17). In the present case, we write the two components of the vector $\underline{f}(y)$ in the form,

$$
f_{A}(y)=\sum_{\alpha} R_{\alpha}^{A} /\left[y-\rho_{\alpha}^{A}\right] \quad(0 \leq y \leq \infty)
$$

and

$$
f_{B}(y)=\sum_{\alpha} R_{\alpha}^{B} /\left[y-\rho_{\alpha}^{B}\right] \quad(-\infty \leq y \leq 0) .
$$

The values of the integrals for $\mathrm{N}_{A}(q)$ and $\mathrm{N}_{B}(q)$ in eqs (B.37) and (B.38) can be obtained from eqs (C.7) and (C.13) by replacing $q$ by $-q$ and not $\iota$ by $-\iota$. The result for $N_{A}(q)$ is identical to that given by eq (B.18) except that the superscript $A$ has to be added to $\underline{R}_{\alpha}$ and $e_{\alpha}$ on the RHS. The result for $\mathrm{N}_{B}(q)$ is

$$
N_{B}(q)=-\frac{\exp (-\pi q)}{E(q)} \sum_{\alpha} R_{\alpha}^{B}\left(\rho_{\alpha}\right)^{-\iota q-0.5}
$$




\section{Appendix I-C}

\section{Evaluation of Integrals}

In this apperidix, we shall evaluate certain integrals by using contour integration. These integrals are required for deriving various formulas in the paper. We shall also obtain the force function and its integral to ensure that the constraint given by eq (12) is satisfied.

First, we shall evaluate the following integral, which is required for calculating the $\mathrm{H}^{\mathrm{A}}$ function from the force function in eq (B.2) or (B.23).

$$
I^{A}(q, p)=\int_{0}^{\infty} \frac{t^{i q-0.5}}{t-\rho} d t
$$

where $\rho$ is complex and $q$ is real.

To evaluate $I^{A}(q, \rho)$, consider the integral of the following complex function of variable $z$ :

$$
z=\frac{z^{i q-0.5}}{z-\rho}
$$

where $\mathrm{z}$ is complex. We integrate $\mathrm{z}$ over the contour shown in figure I-9. The contour consists of an outer circle $C$ with a cut on the positive real axis. The radius of the circle approaches infinity in the limit. The argument of $z$ is taken to be 0 at the upper branch (just above the cut) and $2 \pi$ at the lower branch (just below the cut); that is,

$$
t_{+}=t+\iota \varepsilon=t
$$


and

$$
t_{-}=t-\iota \varepsilon=t \exp (2 \pi \iota),
$$

where $t$ is the real part of $z$.

Thus we obtain from Cauchy's theorem,

$$
\int_{C} \frac{z^{i q-0.5}}{z-\rho} d z+E(q) I^{A}(q, p)=2 \pi \iota \sum \text { Residues }
$$

where

$$
E(q)=1+\exp (-2 \pi q)
$$

The first term in eq (C.5), denoting the contribution of the outer circle, vanishes in the limit when $C$ extends to infinity. since the integrand has only one pole inside the contour at $z=\rho$, we obtain from eq (C.5)

$$
I^{A}(q, \rho)=\frac{2 \pi \iota \rho^{\iota q-0.5}}{E(q)}
$$

In order to calculate the value of $H^{A}(y+\iota \varepsilon)$ and $H^{A}(y-\iota \varepsilon)$, we need the value of $I(q, \rho)$ just above and just below the real axis. Using eq (C.3) and (C.4), we obtain the following values of the integral over the upper and the lower branches of the cut:

$$
I^{A}(q, y+\iota \varepsilon)=\quad \frac{2 \pi \iota y^{\iota q-0.5}}{E(q)}
$$

and

$$
I^{A}(q, y-\iota \varepsilon)=-\frac{2 \pi \iota \exp (-2 \pi q) y^{\iota q-0.5}}{E(q)}
$$


We now evaluate the following integral, which is required for calculating $\mathrm{H}^{\mathrm{B}}(\mathrm{z})$ in eq $(\mathrm{B} .24)$ :

$$
I^{B}(q, p)=\int_{-\infty}^{0} \frac{|t|^{i q-0.5}}{t-\rho} d t
$$

To evaluate $I^{B}(q, p)$, we consider the same function as defined by eq (C.2) and evaluate its integral on the contour given in figure I-10, which has a cut on the negative real axis. The argument of $z$ is taken to be $\pi$ on the upper branch and $-\pi$ on the lower branch so for $-\infty \leq t \leq 0$,

$$
t_{+}=t+\iota \varepsilon=|t| \exp (\pi \iota)
$$

and

$$
t_{-}=t-\iota \varepsilon=|t| \exp (-\pi \iota)
$$

Proceeding as before, we obtain

$$
I^{B}(q, \rho)=-\frac{2 \pi \exp (-\pi q) \rho^{l q-0.5}}{E(q)}
$$

The values of $I^{B}(q, p)$ just above and below the real axis as calculated by using eqs (C.11) - (C.13) are

$$
I^{B}(q, y+\iota \varepsilon)=\frac{2 \pi \iota \exp (-2 \pi q)|y|^{\iota q-0.5}}{E(q)}
$$

and

$$
I^{B}(q, y-\iota \varepsilon)=-\frac{2 \pi \iota|y|^{\iota q-0.5}}{E(q)}
$$


We now evaluate the integral over $q$ required for the calculation of the $\mathrm{H}$ function. As we see from eq (54), we need to evaluate an integral of the form,

$$
I_{H}(z)=\int_{-\infty}^{\infty} \frac{p(q) d q}{E(q) D(q)} z^{l q-0.5},
$$

where $D(q)$ is the determinant of the matrix $M(q)$ and $P(q)$ is an analytical function of $q$ with no poles.

To evaluate the integral in eq (C.16), we choose a semicircular contour either in the UHP or in the LHP as shown in figure I-11. In the present case, the contribution of the integral over the large semicircle vanishes in the UHP if $\bmod (z)>1$ and in the LHP if mod(z) < 1. Accordingly, we choose the contour in the UHP if $\bmod (z)>1$ and in the LHP if $\bmod (z)<1$.

Let $q_{n}$ and $Q_{r}$ denote the zeroes of $E(q)$ and $D(q)$ respectively. The integrand in eq (C.16) has two sets of poles; those at $q_{n}$ will be called set $I$ and those at $Q_{r}$ set II. Thus

$$
q_{n}=(n+0.5) i
$$

where $\mathrm{n}$ is an integer- which can be positive, negative or zero and $Q_{r}$ are the roots of the determinantal equation,

$$
D\left(Q_{r}\right)=0
$$

We assume that all $Q_{r}$ are distinct, which should be generally true. When they are not, it would be a higher order pole which can be treated by the standard methods of contour integration. Moreover, in general, some $q_{n}$ will be equal to some $Q_{r}$. These second-order poles can also be treated by standard methods. 
However, presently our object is to write a general expression for the integral with the view to identify its singularities and to obtain an expression for the force function and its integral. For this purpose, it would be convenient to introduce a small positive imaginary part $\iota \varepsilon$ in $q$ in the denominator of the RHS of eq (C.16) as follows and, in the end, take the limit $\varepsilon=0$.

$$
I_{H}(z)=\int_{-\infty}^{\infty} \frac{P(q) d q}{E(q-i \varepsilon) D(q+i \varepsilon)} z^{i q-0.5}
$$

The two sets of poles in the integrand in eq (c.19) can now be written as follows:

Set I. Zeroes of $\mathrm{E}(\mathrm{q})$.

$$
q=q_{n}+i \varepsilon
$$

Set II. Zeroes of $D(q)$.

$$
q=Q_{r}-i \varepsilon
$$

The allowed values of $q_{n}$ and $Q_{r}$ depend upon whether the contour of integration (see figure I-11) has been chosen to be in the UHP or LHP and are given below:

I. $\operatorname{Mod}(z)>1$ (Contour in UHP).

$$
\mathrm{n}=0,1,2, \ldots
$$

and

$$
\operatorname{Im}\left(Q_{r}\right)>0
$$

II. $\operatorname{Mod}(z)<1$ (Contour in LHP). 


$$
\mathrm{n}=-1,-2,-3, \ldots
$$

and

$$
\operatorname{Im}\left(Q_{r}\right)<0
$$

The residue of $E(q-i \varepsilon)$ at $q_{n}$ is $2 \pi$ and that of $D(q+i \varepsilon)$ at $Q_{r}$ is $D^{\prime}\left(Q_{r}\right)$, where the prime denotes differentiation with respect to the argument. Thus, we obtain the following value of the integral by using Cauchy's integration formula:

$I_{H}(z)= \pm \iota \sum_{n} \frac{P\left(q_{n}+i \varepsilon\right) z^{-(n+1)-\varepsilon}}{D\left(q_{n}+2 i \varepsilon\right)}$

$\pm 2 \pi \iota \sum_{r} \frac{P\left(Q_{r}-i \varepsilon\right) z^{i Q_{r}-0.5+\varepsilon}}{E\left(Q_{r}-2 \iota \varepsilon\right) D^{\prime}\left(Q_{r}\right)} \quad \pm R(z, \varepsilon)$

where the sums in eq (C.24) are over those values of $q_{n}$ and $Q_{r}$ which are not degenerate, that is, which represent only a single-order pole; and $\mathrm{R}(z, \varepsilon)$ denotes the contribution of those values of $\mathrm{n}$ and $\mathrm{r}$ which represent a pole of second or higher order in the integrand. The plus signs in eq (C.24) are to be taken when $\bmod (z)>1$ and negative when $\bmod (z)<1$.

In the cases considered in section $3 A$ and $3 B, D(q)$ is zero at the following two values of $q$ which are part of set $I$ :

$$
q_{0}=Q_{0}=\iota / 2
$$

and

$$
q_{-1}=Q_{-1}=-\iota / 2,
$$

which correspond respectively, to $n=0$ and -1 in eq (C.17). These values therefore represent second-order poles which will contribute, respectively, when the contour is chosen in UHP and 
LHP. From eqs (C.22) and (C.23) the pole at $q_{0}$ will contribute when $\bmod (z)>1$ and the one at $q_{-1}$ will contribute when $\bmod (z)<$ 1. We shall now evaluate $R_{H}(z, \varepsilon)$ at these poles.

First we consider the former case, when $\bmod (z)>1$. We use the expansion formulas,

$$
E\left(q_{0}-2 \iota \varepsilon\right)=-4 \pi \iota \varepsilon(1+2 \pi \iota \varepsilon)
$$

and

$$
D\left(q_{0}+2 \iota \varepsilon\right)=2 \iota \varepsilon D^{\prime}\left(q_{0}\right)\left[1+\iota \varepsilon D^{\prime \prime}\left(q_{0}\right) / D^{\prime}\left(q_{0}\right)\right]
$$

Thus, we obtain the following for $\bmod (z)>1$ :

$\operatorname{Lim}_{\varepsilon \rightarrow 0} R_{u}(z, \varepsilon)=\frac{\iota z^{-1}}{D^{\prime}\left(q_{0}\right)}\left[P^{\prime}\left(q_{0}\right)+\iota P\left(q_{0}\right) \operatorname{Ln}(z)\right.$

$$
\left.-\frac{1}{2} P\left(q_{0}\right) D^{\prime \prime}\left(q_{0}\right) / D^{\prime}\left(q_{0}\right)+\pi P\left(q_{0}\right)\right] \text {. }
$$

The corresponding expression for $R(z, \varepsilon)$ when $\bmod (z)<1$ is formally similar to eq (C.29) and is written as

$\begin{aligned} \operatorname{Lim}_{\varepsilon \rightarrow 0} R_{1}(z, \varepsilon)= & \frac{\iota}{D^{\prime}\left(q_{0}\right)}\left[P^{\prime}\left(q_{-1}\right)+\iota P\left(q_{-1}\right) \operatorname{Ln}(z)\right. \\ & \left.-\frac{1}{2} P\left(q_{-1}\right) D^{\prime \prime}\left(q_{-1}\right) / D^{\prime}\left(q_{-1}\right)+\pi P\left(q_{-1}\right)\right]\end{aligned}$

We now calculate the integral required for the force functions. An explicit knowledge of these functions is not required for calculating the Green's functions for which, as we 
see from eqs (116) and (117), a knowledge of H-functions is sufficient. However, we do need to calculate the integral of the force functions in order to satisfy the constraint given by equation (12).

Using the Plemelj relation, as given by eq (B.42), we see that the two force functions can be written in terms of an integral of the form,

$$
I_{F}(y)=\int_{-\infty}^{\infty} \frac{E(q) P(q) d q}{E(q-i \varepsilon) D(q+i \varepsilon)}|y|^{i q-0.5}
$$

In case of the force function in the LHP, the numerator of the integrand in eq (C.31) will have a factor exp( $\pi q)$ which can be easily included in the result derived below. The integral in eq (C.31) can be evaluated by using the same method as described earlier. The result is

$$
\begin{aligned}
I_{F}(y) & =-2 \pi \varepsilon \sum \frac{P\left(q_{n}+i \varepsilon\right)|y|^{-(n+1)-\varepsilon}}{D(q+2 i \varepsilon)} \\
& +2 \pi \iota \sum_{Y} \frac{E\left(Q_{Y}-\iota \varepsilon\right) P\left(Q_{Y}-i \varepsilon\right)|Y|^{i Q_{Y}-0.5+\varepsilon}}{E\left(Q_{Y}-2 \iota \varepsilon\right) D^{\prime}\left(Q_{Y}\right)}+R(Y / \rho, \varepsilon),
\end{aligned}
$$

where the allowed values of $\mathrm{n}$ and $\mathrm{r}$ are defined by eqs (C.22) and (C.23), and $R(Y / \rho, \varepsilon)$ accounts for any second order poles as the corresponding $R$ term in eq (C.24). The complex variable $\rho$ is contained in the function $\underline{P}(q)$.

The first term on the RHS of eq (C.32) (along with the last term where it exists) gives the particular solution of eqs (25) 
and (26). The second term is a solution of the homogeneous equation since $D\left(Q_{r}\right)$ is zero for all $Q_{r}$. The first term on the RHS of eq (C.32) is vanishingly small in the limit $\varepsilon=0$. However, this term can-not be neglected because it gives a finite contribution to $\mathrm{H}(z)$, as we can see from eqs (C.19) and (C.24). The $1 / y$ term which corresponds to $n=0$ or $q=\iota / 2$, is actually a delta-function term which is zero for all values of $y$ except $y=0$. This is similar to the term in eqs (158) and (159) for the antiplane-strain mode.

The integral of the force function in the $q$ representation can be directly obtained from eq (c.32). We write the total integral of the force function as a sum of those for the UHP and the LHP as

$$
\underline{I}_{t}=\underline{I}^{A}+\underline{I}^{B},
$$

where

$$
\begin{aligned}
I^{A} & =\int_{y_{0}} I_{F}^{A}(y) d y \\
& =\int_{-\infty}^{\infty} \frac{p^{A}(q) d q}{D(q)(i q+0.5)}\left[y_{\infty}^{i q+0.5}-y_{0}^{i q+0.5}\right],
\end{aligned}
$$

with a similar expression for $\underline{I}^{B}$. The corresponding limits on the $y$ integration in $\underline{I}^{B}$ will be from $-y_{\infty}$ to 0 . In the end we shall take the limits $y_{0}=0$ and $y_{\infty}=\infty$. 
Now we evaluate the q-integral by using the contour integration as in the case of integrals in eqs (C.19) and (C.31). First, consider the integral of the lower $y$ limit, the term containing $Y_{0}$. In this limit, as discussed earlier in this appendix, we have to take the contour in the LHP as shown in figure I-11. Thus we find that the integral will contain only positive powers of $y_{0}$ and therefore will be zero in the limit $y_{0}=$ 0 .

Now consider the integral of the term containing $Y_{\infty}$. In this case we have to choose the contour in the UHP. We have to include the residue at $Q_{r}$, the zeroes of $D(q)$, and at $q_{0}=\iota / 2$, which arises from the factor $(q-\iota / 2)$ in the denominator. For the moment, ignore the pole at $q_{0}=\iota / 2$ The integral will then contain terms with $\left(\left\llcorner Q_{r}+0.5\right)\right.$ as powers of $Y_{\infty}$. In this case, as given by eqs (C.17) and (C.18), Im $\left(Q_{r}\right) \geq 0$. Obviously the terms for which Im $\left(Q_{r}\right) \geq 0.5$ will also vanish in the limit $Y_{\infty}=\infty$. Hence the only terms which will contribute to the integral are those for which

$$
0 \leq \operatorname{Im}\left(Q_{\Gamma}\right)<0.5
$$

Now we consider the special term for which $q=\iota / 2$ for which the exponent of $Y_{\infty}$ is 0 . As shown in section $3 A$, the value of $D(q)$ is also zero at $q=\iota / 2$. However it does not represent a second-order pole because, as given by eq (97), $P(q)$ is also zero at $q=$ l/2. Therefore, the integrand in eq (C.34) has only a first-order pole at $q_{0}=\iota / 2$. The contribution of this pole to the integral can be written as

$$
I_{0}^{A}=2 \pi \operatorname{Lim}_{q=\iota / 2}\left[\frac{P^{A}(q)}{D(q)}\right]
$$

Similarly, we obtain the following expression for the integral of the force on the negative $Y$ axis: 


$$
I_{0}^{B}=2 \pi \operatorname{Lim}_{q=\iota / 2}\left[\frac{p^{B}(q)}{D(q)}\right] .
$$

Adding eqs (C.36) and (C.37), and using eqs (93) and (94) in the definition of $\underline{P}(q)$ as given by eq (55), we find that the numerator of the limit will approach zero faster than the denominator. The total contribution to the integral from the pole at $q_{0}=\iota / 2$ will, therefore, be zero.

For satisfying eq (12), the only contribution to $I_{t}$ can therefore come from the poles at $q=Q_{r}$, where $Q_{r}$ is subject to the constraint given by eq (c.35). Hence, we include only these contributions, so

$$
\left.\underline{I}^{A}=2 \pi \iota \sum_{Q_{r} \neq l / 2} \frac{P^{A}\left(Q_{r}\right)}{D^{\prime}\left(Q_{r}\right)\left(Q_{r}+1 / 2\right)} y_{\infty}^{\iota Q_{r}-0.5}\right],
$$

with a similar expression for $\underline{I}^{B}$. The total integral of the force function is given by eq (C.33).

If a $Q_{r}$ does not exist which satisfies (C.34), then $I_{t}$ is zero and eq (12) is satisfied. If $I_{t}$ is nonzero, then additional force functions have to be applied to the solid corresponding to the same $Q_{r}$ which give a finite contribution in eq (c.38) so that eq (12) is satisfied. These terms will give zero $\tau_{i 1}$ at $x=0$, since they are the solution of the homogeneous Hilbert problem.

The expression on the RHS of eq (C.38) diverges for $Y_{\infty}=\infty$. The singularity will cancel from the force integral calculated from the final solution. The force integral will be zero, since we have ensured that eq (12) is satisfied by the final solution. 


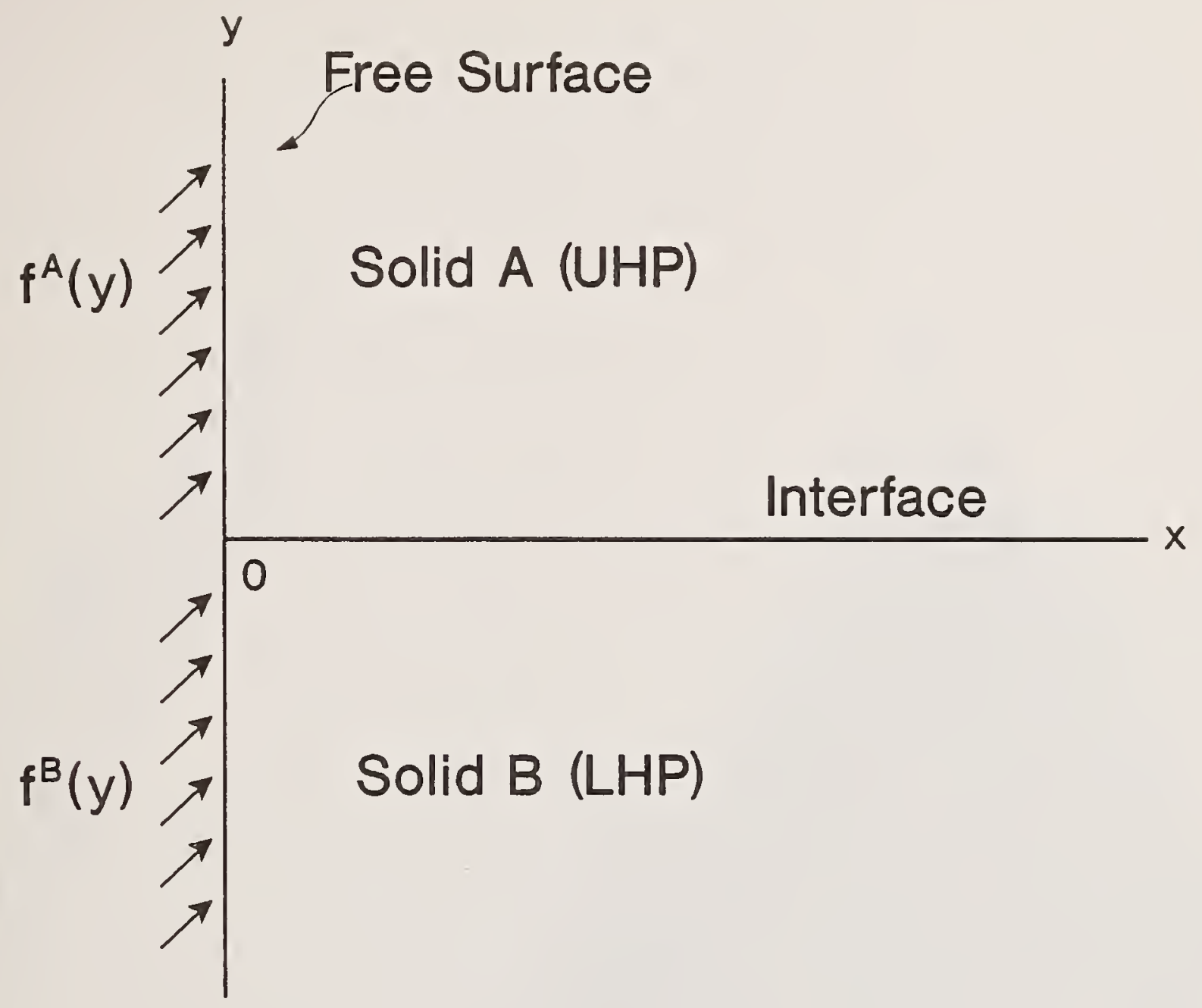

Figure I-1: A bimaterial composite containing a free surface normal to the interface and the coordinate system used in these calculations. The force functions $\mathrm{F}^{\mathrm{A}}(\mathrm{y})$ and $\mathrm{F}^{\mathrm{B}}(\mathrm{y})$ are applied just outside the free surface. 


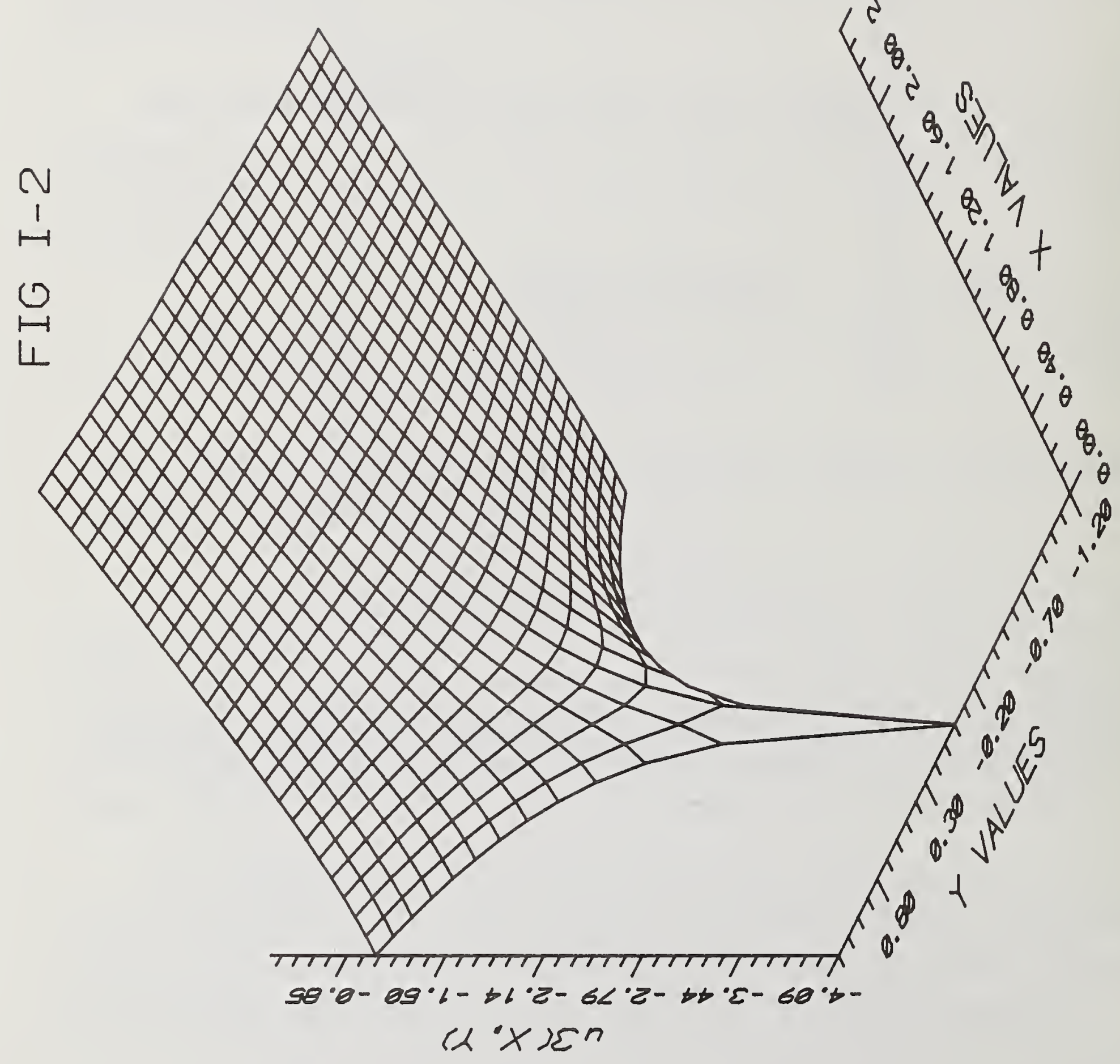

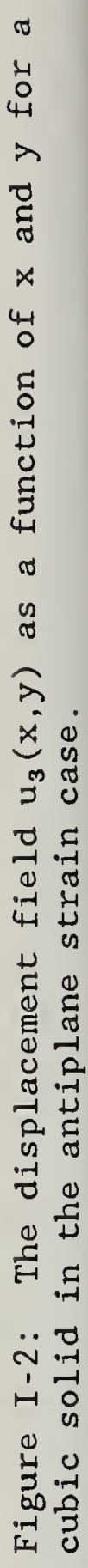


$M$
1
$\mapsto$
0
-
$\sqcup$

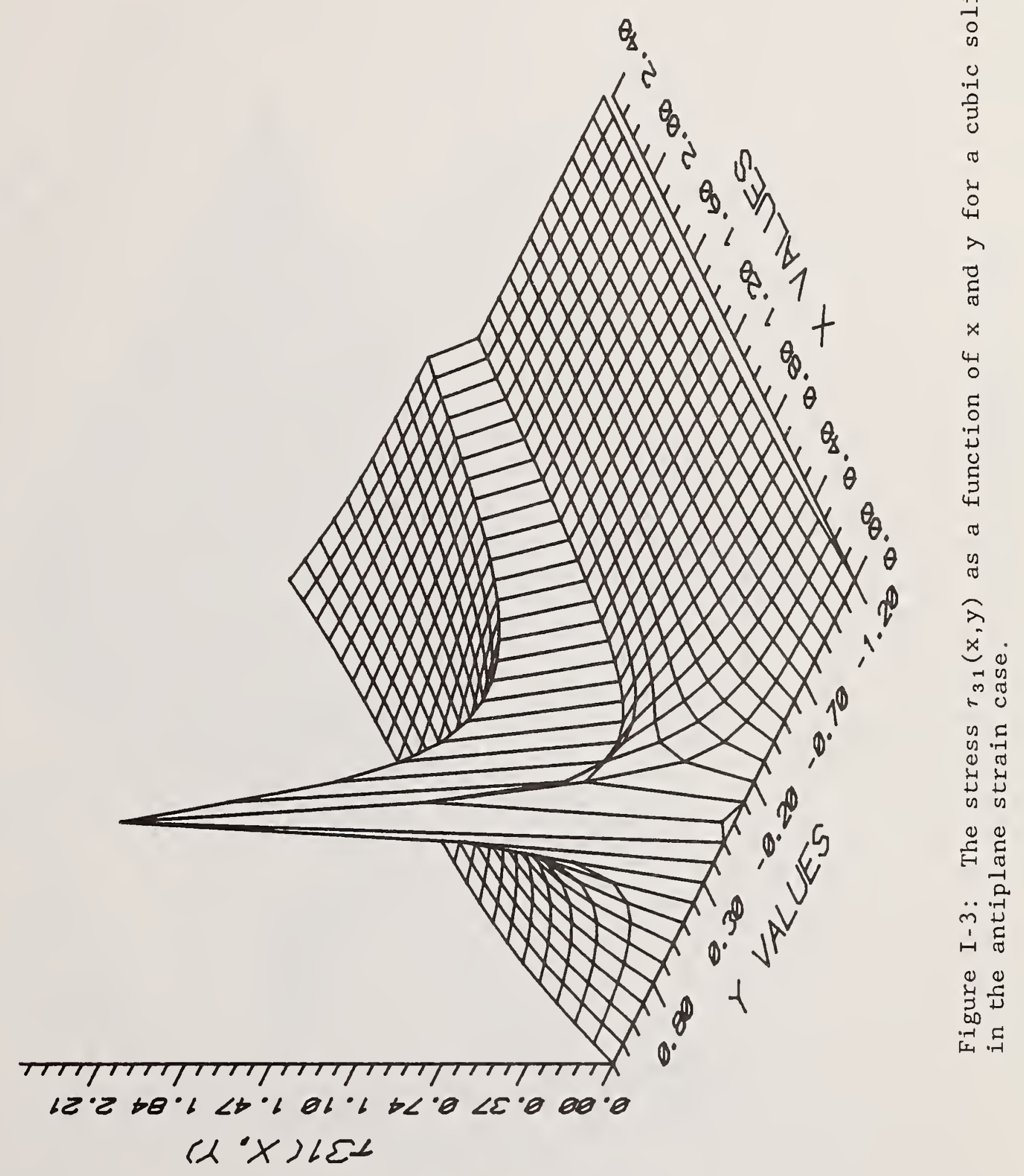




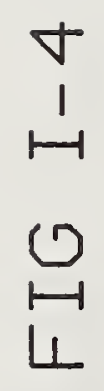

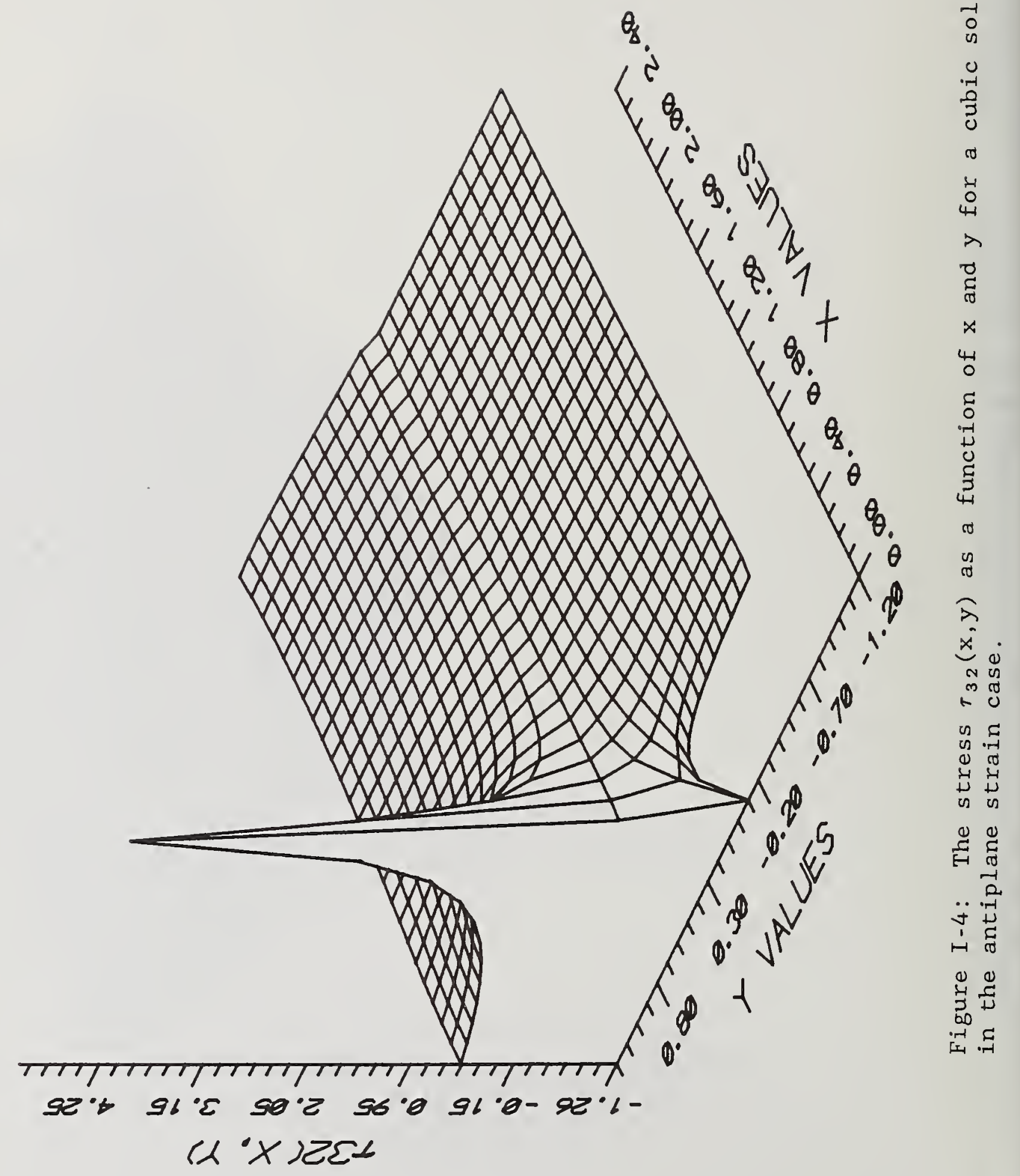




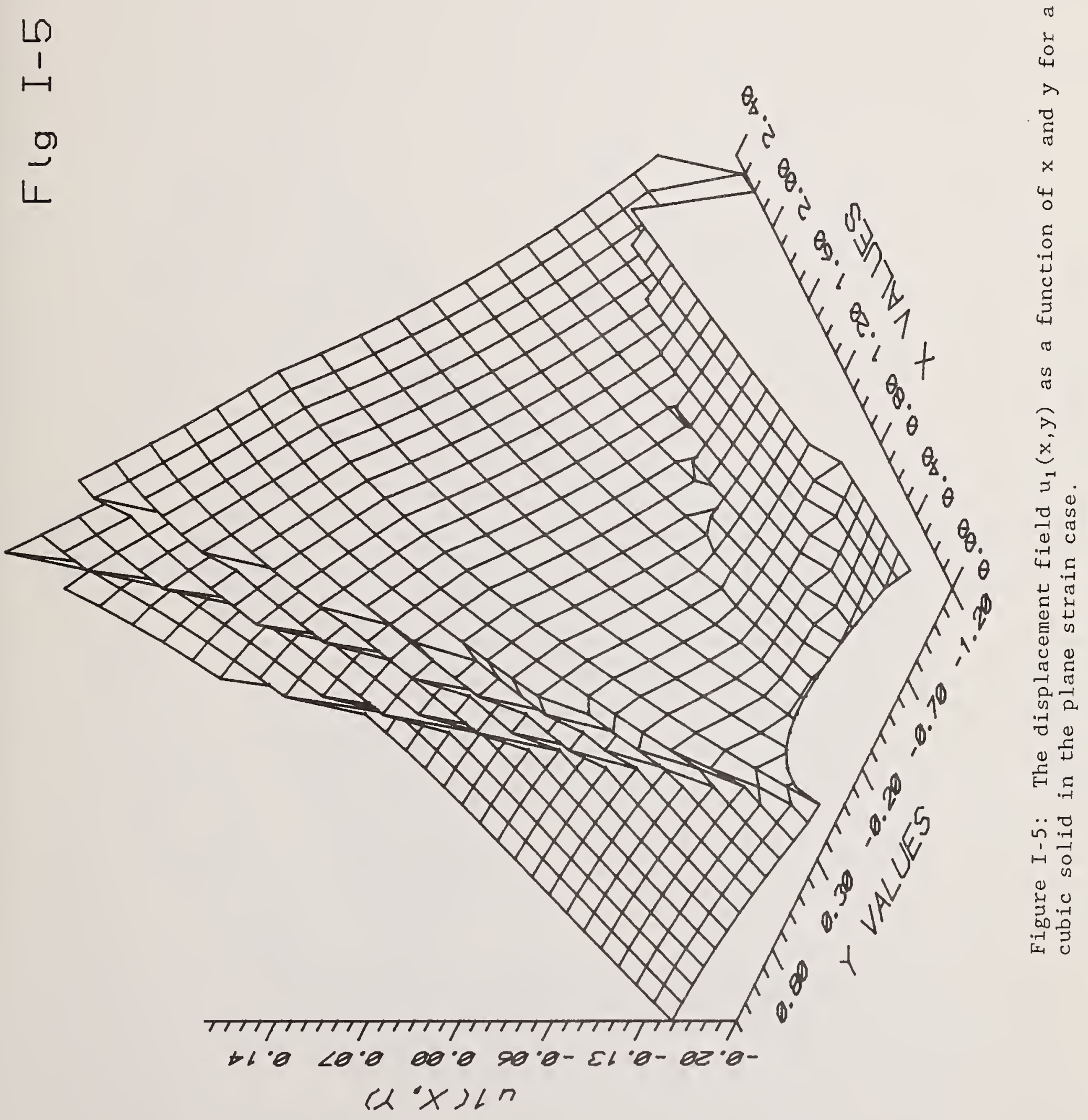




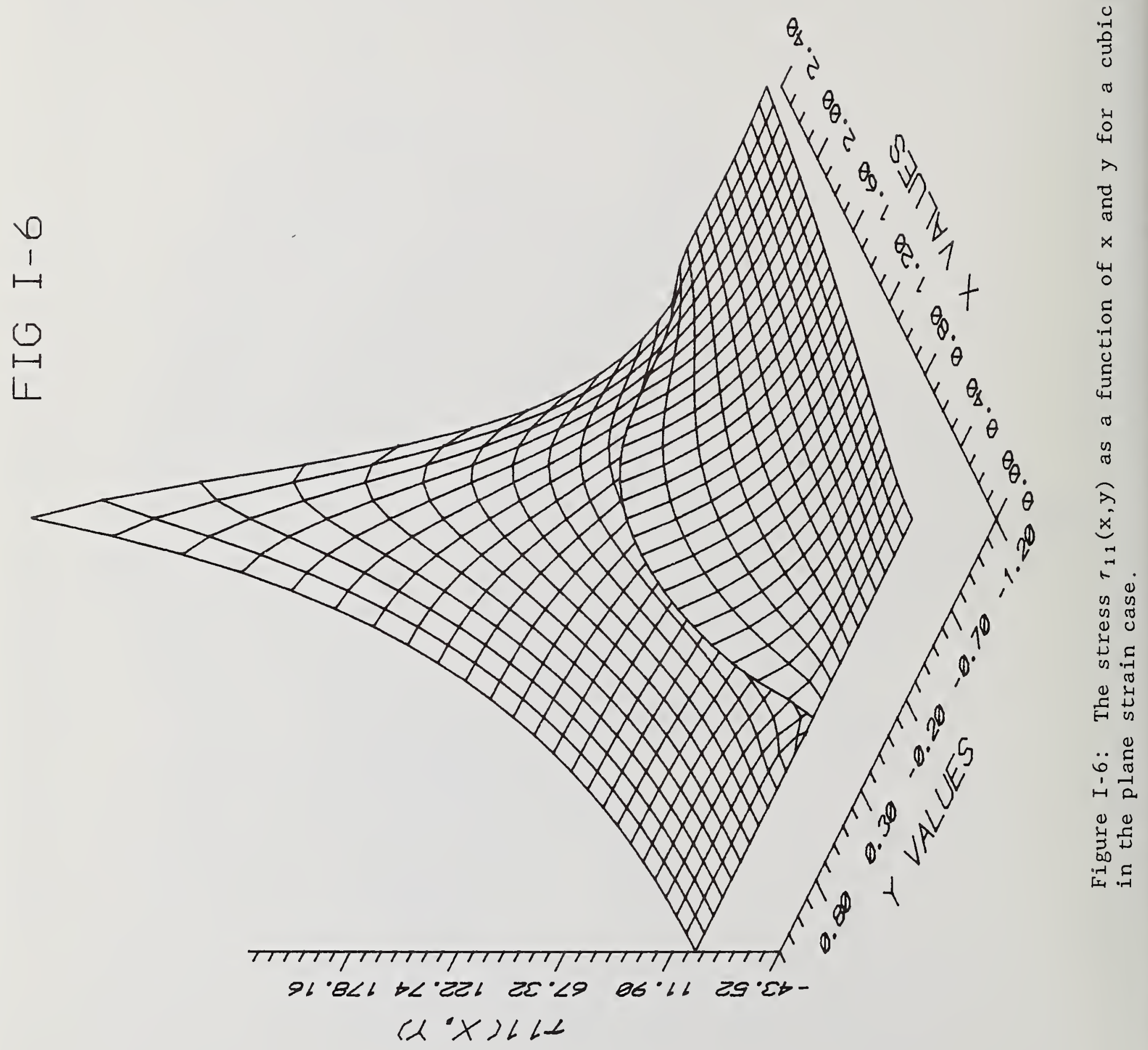




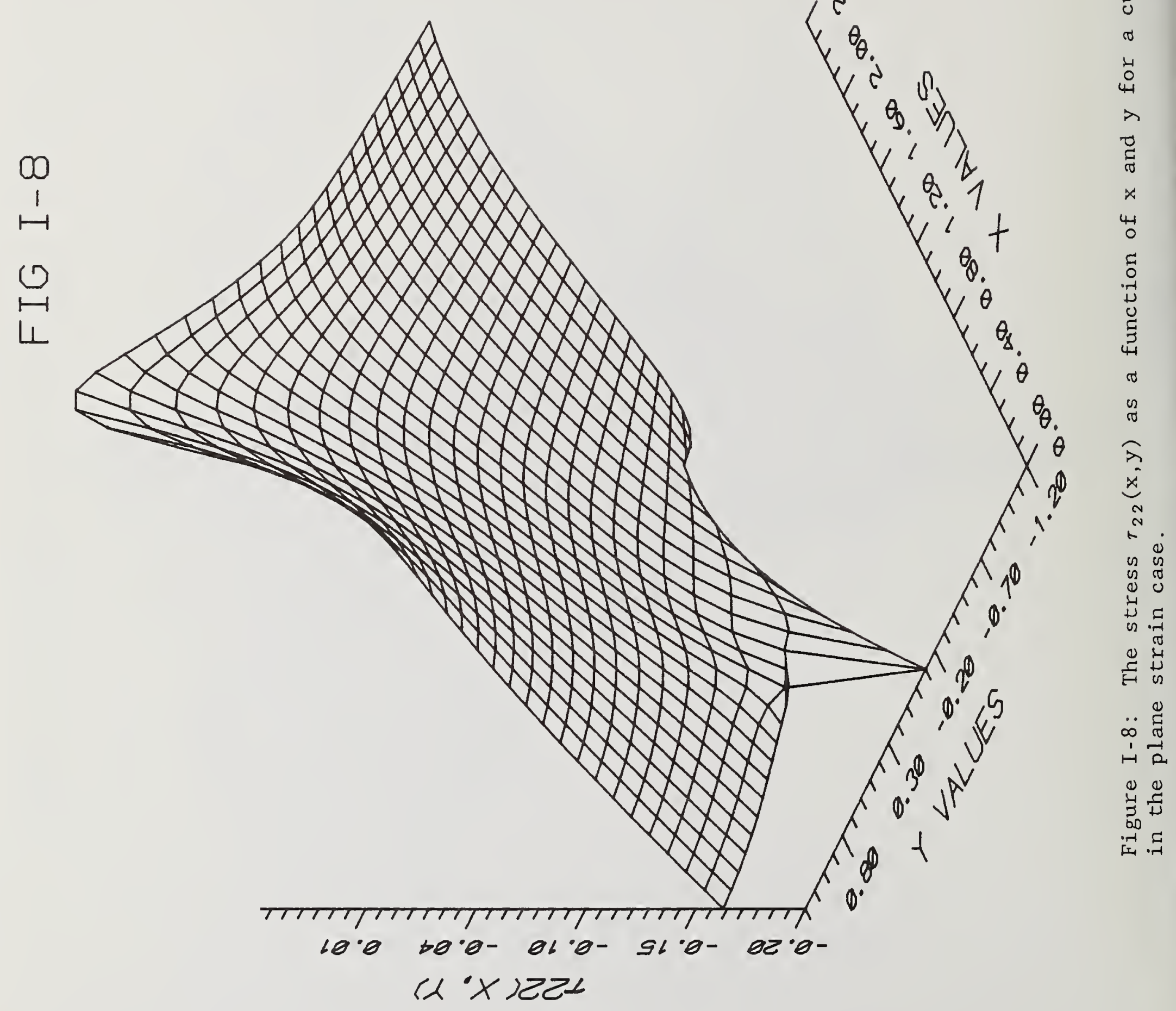




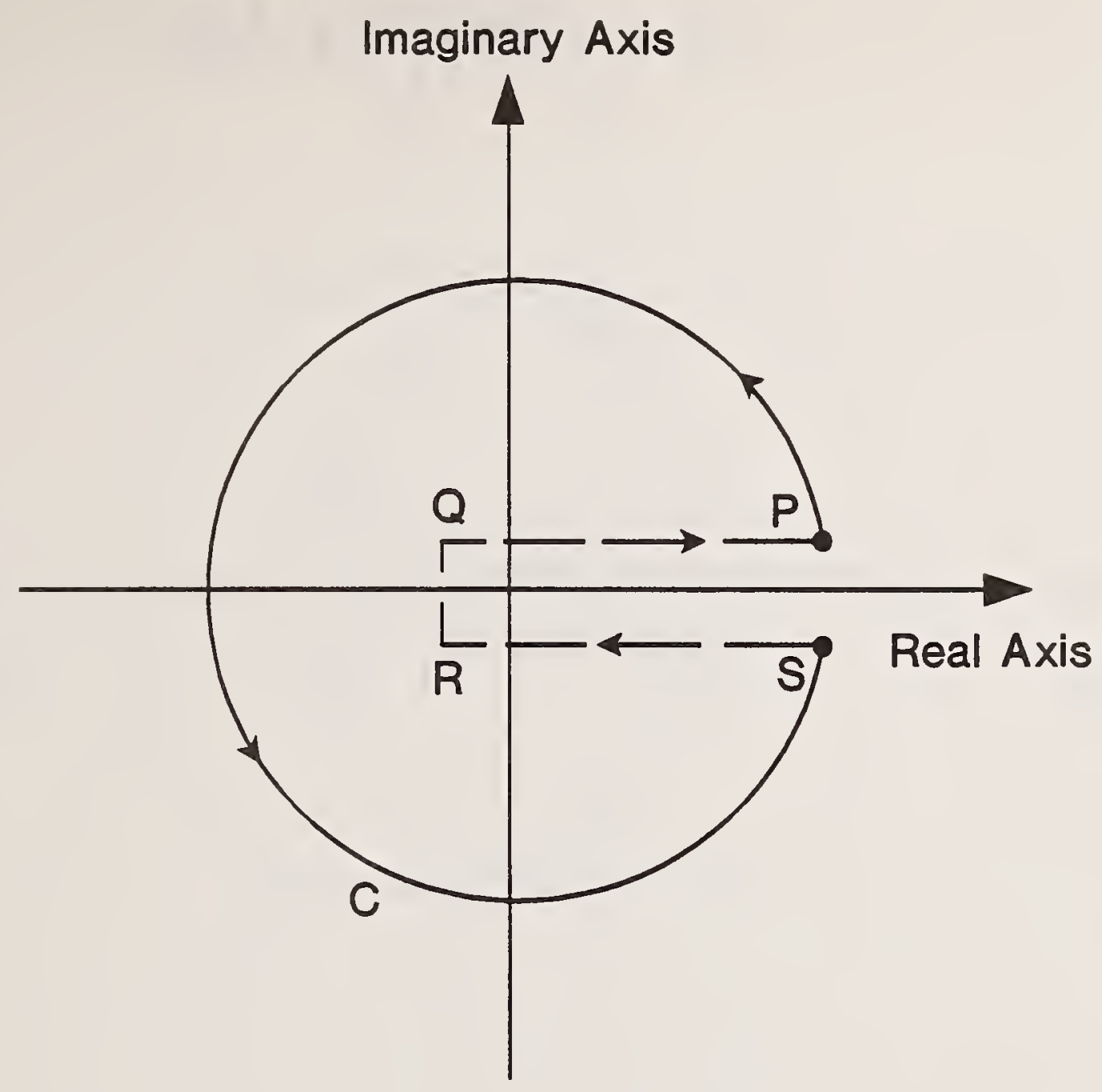

Figure I-9: The contour used for evaluation of the integral for the function $\mathrm{H}^{\mathrm{A}}(\mathrm{z})$. The cut $\mathrm{PQRS}$ is along the positive real axis. The phase angle is 0 on the branch $P Q$ and $2 \pi$ on the branch RS. 


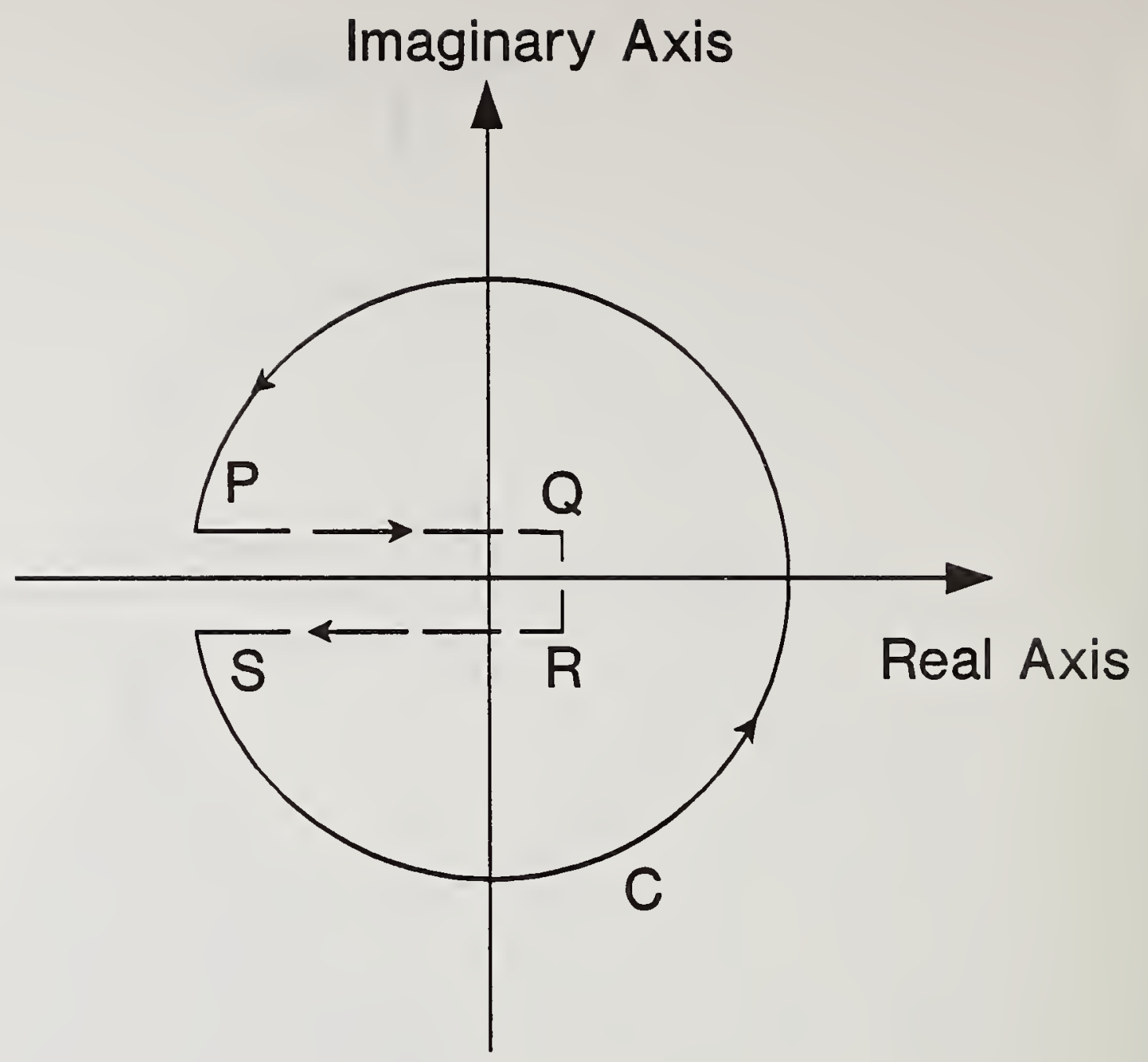

Figure I-10: The contour used for evaluation of the integral for the function $H^{B}(z)$. The cut $P Q R S$ is along the negative real axis. The phase angle is $\pi$ on the branch $\mathrm{PQ}$ and $-\pi$ on the branch RS. 


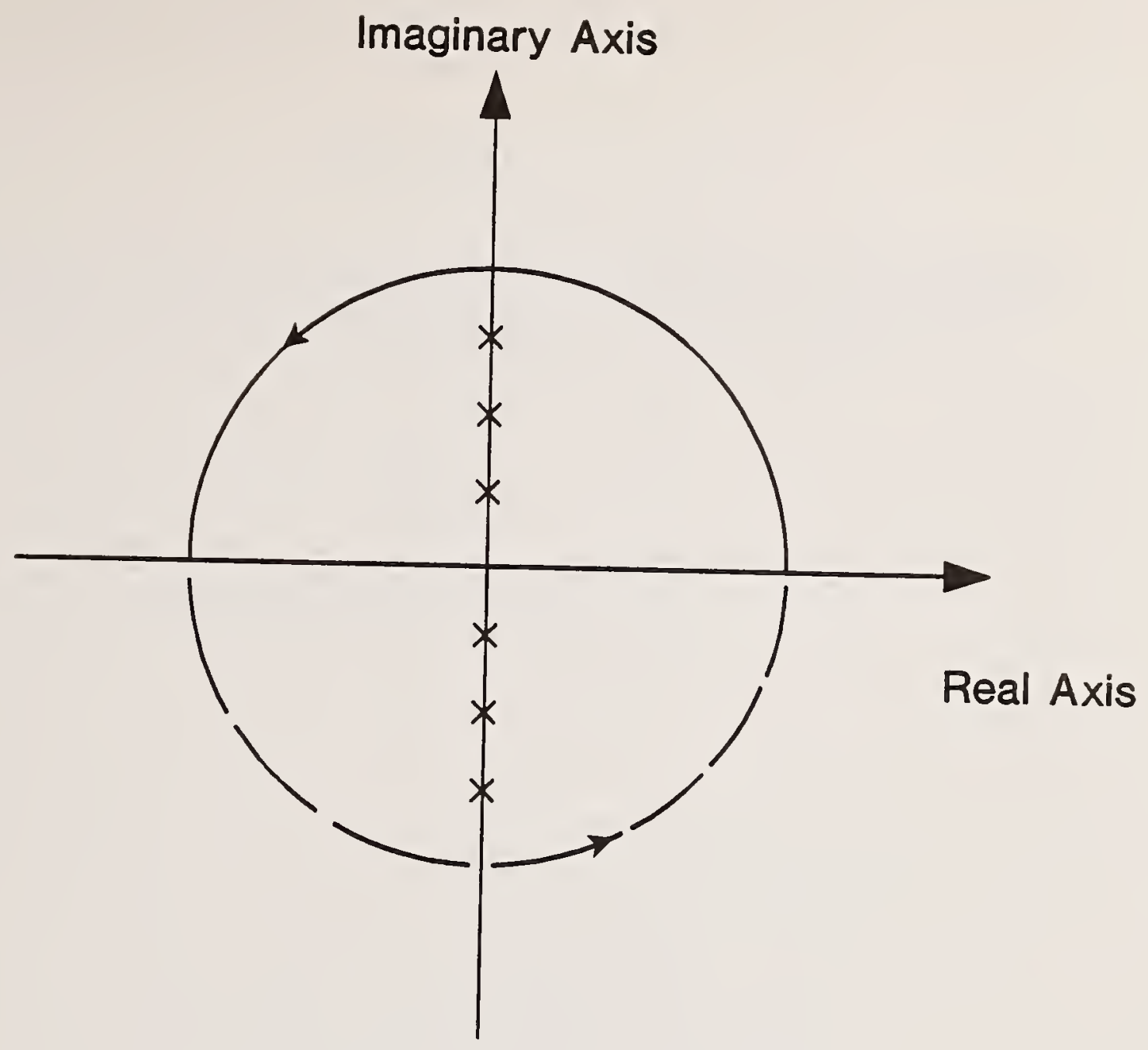

Figure I-11: The semicircular contours used for evaluation of the q-integrals in the UHP (solid line; for mod $z>1$ ) and in the LHP (dotted line; for $\bmod z<1$ ). 

Part II

Generalized Plane-Strain Analysis of a Bimaterial Composite Containing a Free Surface Normal to the Interface

by

V.K.Tewary and R.D. Kriz 


\section{Introduction}

In this part, we extend the elastic plane-strain Green's function calculated in Part I to account for the generalized plane strain. We apply it to calculate the stress and the displacement field in a bimaterial composite containing a free surface normal to the interface which is subjected to generalized plane strain. The generalized plane strain is induced by subjecting the solid to an out-of-plane load such that the strain component $\varepsilon_{33}$ is constant.

The result is obtained in terms of a closed integral representation, which is evaluated analytically as well as numerically. As in part $I$, the analytical evaluation of the integral leads to a series representation--not necessarily convergent--of the stress from which the singularities in the stress can be identified precisely. The numerical calculation of the integral representation does not suffer from convergence problems. It contains singular as well as nonsingular terms and gives reliable values of the stress and the displacement field in the entire solid. The method is applied to a cubic solid containing a $\Sigma-5$ grain boundary and to fiber-reinforced laminated composite with different relative fiber orientations.

In general, the nature of singularities in the generalized plane-strain problem is similar to that obtained in Part I. The power of a singularity depends upon the elastic constants of the constituent materials, whereas its weight depends upon the nature of the loading as well as the elastic constants. In some cases the singular term may also have a logarithmic oscillatory factor.

The plan of this part is as follows. In section 2, we reformulate the Green's function method for generalized plane-strain problems and apply it to calculate the displacement 
and stresses in an infinite (without a free surface) bimaterial composite subjected to an out-of-plane load. The displacement and the stress components $\tau_{i 2}$ thus calculated satisfy the required continuity conditions at the interface. We then use these results and the technique given in Part I to calculate the stress and the displacement field in a similarly loaded composite which contains a free surface. These calculations are given in section 3 . The analysis is applied to a $\Sigma-5$ tilt grain boundary in a cubic solid in section 4 and to laminated composites in section 5. A summary and a discussion of the results are presented in section 6 .

\section{Displacement and the Stress Field in an Infinite Composite} Subjected to Generalized Plane Strain

In this section we calculate the displacement and the stress field in an infinite bimaterial composite which has no free surfaces. A schematic representation of the model solid considered in this section is given in figure II-1, which also shows the coordinate axes. This model is same as used in Paper I. The notation and the coordinate axes used in this paper are the same as in Part I. The notation in this paper is mostly the same as used in Paper $I$.

The displacements in both UHP and LHP have to satisfy the following equation of elastic equilibrium (add superscripts $A$ or $B$ corresponding to UHP or LHP respectively):

$$
c_{i k j l} \frac{\partial^{2} u_{j}}{\partial x_{k} \partial x_{l}}=0
$$

Equation (1) is the homogeneous part of the elastic equilibrium equation valid in the absence of any body forces. As 
shown in Paper I, its solution in a particular region can be written using the elastic Green's function as

$$
u(x)=\int_{L} G\left(x, x^{\prime}\right) f\left(x^{\prime}\right) d x^{\prime}
$$

where $f\left(x^{\prime}\right)$ is an arbitrary function which is applied in a region $L$ such that the region $L$ is outside the region of solution of eq (1). The integration in eq (2) is carried out over the entire region $L$. The function $f\left(x^{\prime}\right)$ in eq (2) is usually referred to as the force function. The force function in eq (2) does not represent a physical force on the solid. It is a mathematical artifact which is used to satisfy the prescribed boundary conditions.

In the plane-strain problem, $u(x), G\left(x, x^{\prime}\right)$ and $f\left(x^{\prime}\right)$ are functions of only the $x_{1}$ - and $x_{2}$ - coordinates and are independent of the $x_{3}$ - or the $z-$ coordinate. Even in plane-strain problems, the $\mathrm{z}$ - components of these quantities, in general, will not be zero. The solution of eq (1) using eq (2) for the plane-strain case has been discussed in Paper I.

In the generalized plane-strain problem, the solid is subjected to a load in the $z$ - direction such that the $\varepsilon_{33}$ is constant everywhere in the solid and has a prescribed value, $\varepsilon$ being the strain tensor. The displacement field in the solid can therefore be written in the form

$$
u_{\infty i}(\underline{X})=\eta \delta_{i 3} x_{3}+u_{p i}(\underline{x}),
$$

where $\eta$ is the prescribed value of $\varepsilon_{33}$ so

$$
\eta=\varepsilon_{33}
$$


is constant and $\underline{u}_{p}(\underline{x})$ is a vector which is a function of only $x_{1}$ and $x_{2}$ and not of $x_{3}$. The subscript $\infty$ on $\underline{u}$ indicates that it denotes the displacement field in an infinite composite with no free surfaces. Similarly, the subscript $p$ on $u$ on the RHS indicates that it is a solution of the plane-strain problem as described below.

Following the notation used in Part $I, \underline{x}, \underline{x}^{\prime}$ will denote a $2 \mathrm{D}$ (2 dimensional) vector on the $X Y-$ plane with Cartesian components $\mathrm{x}_{1}, \mathrm{x}_{2}$ and $\mathrm{x}_{1}^{\prime}{ }_{1} \mathrm{x}_{2}{ }_{2}$ respectively. The $3 \mathrm{D}$ vectors will be denoted by the upper case variables $X, X^{\prime}$. For example, the position vector $\underline{x}$ in eq (3) denotes a $3 D$ vector with cartesian components $x_{1}, x_{2}$ and $x_{3}$. Our object in this section is to determine $\underline{u}_{p}(\underline{x})$ such that $\underline{u}_{\infty}(x)$ is a solution of eq (1) and it satisfies the following boundary conditions,

and

$$
u_{\infty i}^{A}\left[x_{1}, 0\right]=u_{\infty i}^{B}\left[x_{1}, 0\right] \quad\left(-\infty \leq x_{1} \leq \infty\right)
$$

$$
\mathrm{T}_{\infty 2 i}^{\mathrm{A}}\left[\mathrm{x}_{1}, 0\right]=\mathrm{T}_{\infty 2 \mathrm{i}}^{\mathrm{B}}\left[\mathrm{x}_{1}, 0\right] \quad\left(-\infty \leq \mathrm{x}_{1} \leq \infty\right),
$$

where $T_{-\infty 2}$ is a vector which is defined in terms of the components $\tau_{i 2}$ of the stress tensor (for superscripts $A$ and $B$ ) as

$$
T_{\infty 2 i}=\tau_{i 2}
$$

The stress components can be calculated from the displacement field by using the formulas,

$$
\tau_{i 1}=c_{i 11 k} \frac{\partial u_{k}}{\partial x_{1}}+c_{i 12 k} \frac{\partial u_{k}}{\partial x_{2}}+c_{i 13 k} \frac{\partial u_{k}}{\partial x_{3}},
$$




$$
\tau_{i 2}=c_{i 21 k} \frac{\partial u_{k}}{\partial x_{1}}+c_{i 22 k} \frac{\partial u_{k}}{\partial x_{2}}+c_{i 23 k} \frac{\partial u_{k}}{\partial x_{3}},
$$

and

$$
\tau_{i 3}=c_{i 31 k} \frac{\partial u_{k}}{\partial x_{1}}+c_{i 32 k} \frac{\partial u_{k}}{\partial x_{2}}+c_{i 33 k} \frac{\partial u_{k}}{\partial x_{3}} .
$$

The first term in eq (3) is a constant with respect to the differential operators in eq (1). It would therefore automatically satisfy eq (1). Since $\underline{u}_{p}(\underline{x})$ depends only on $x_{1}$ and $x_{2}$, we can use the plane-strain Green's function obtained in Paper I to determine this part of the displacement field.

First we consider the displacement field in the solid $A$ in the UHP. Following the method given in Paper $I$, we apply hypothetical forces just outside the region $A$ at the set of points $\left(x_{1},-\varepsilon\right)$ where $x_{1}$ varies from $-\infty$ to $+\infty$ and $\varepsilon$ is a small positive constant which will be taken to be zero in the limit. The positive value of $\varepsilon$ ensures that the hypothetical forces are applied just outside the region of solution. We denote this force function by $\underline{f}^{A}\left(x_{1}\right)$. By using eq (2) and as given in Paper $I$, we can write $\underline{u}_{p}(\underline{x})$ in the form,

$$
\underline{u}_{p}^{A}(\underline{x})=\int_{-\infty}^{\infty} \underline{G}^{A}\left(\underline{x}_{i} x_{1}^{\prime},-\varepsilon\right) \underline{\underline{f}}^{A}\left(x_{1}^{\prime}\right) d x_{1}^{\prime},
$$

where $\underline{G}^{\mathrm{A}}\left(\underline{x}_{i} \underline{x}^{\prime}\right)$ denotes the Green's function for the solid A when the solid $B$ is not attached to it.

As described in the previous paragraph, the $Y$ component of $x^{\prime}$ has been taken to be $-\varepsilon$ to ensure that the force $\underline{f}^{A}\left(x_{1}^{\prime}\right)$ has been applied just outside the region A. Equation (11) then gives a solution of eq (1) in the entire space of $A$. 
The Green's function for the solid A, in the absence of solid B, can be written as follows ( see, for example, Paper I):

$$
G^{A}\left(\underline{x} ; \underline{x}^{\prime}\right)=-\frac{1}{\pi} \sum_{\alpha} \underline{\gamma}^{A}(\alpha) \ln \left(z_{\alpha}^{A}-\rho_{\alpha}^{A}\right)
$$

where

$$
\begin{aligned}
& z_{\alpha}^{A}=x_{1}+p_{\alpha}^{A} x_{2}, \\
& \rho_{\alpha}^{A}=x_{1}^{\prime}+p_{\alpha}^{A} x_{2}^{\prime}
\end{aligned}
$$

and $\underline{\gamma}^{A}(\alpha)$ and $p_{\alpha}^{A}$ have been defined by eqs (A.17) and (A.19) respectively in Appendix I-A of Part I. From eqs (3) and (12) we obtain

$$
\underline{u}_{-\infty}^{A}(\underline{x})=\underline{u}_{0}-\frac{1}{\pi} \sum_{\alpha} \underline{\gamma}^{A}(\alpha) \int_{-\infty}^{\infty} \ln \left(z_{\alpha}^{A}-t-\iota \varepsilon\right) \underline{f}^{A}(t) d t,
$$

where it is understood that only the real part of the RHS in eq (15) represents the displacement field and where

$$
u_{0 i}=\eta \delta_{i 3} x_{3}
$$

In the argument of the logarithmic function on the RHS of eq (15) we have written $p_{\alpha}^{A} \varepsilon=\iota \varepsilon$. This is because we have to put $\varepsilon=$ 0 in the limit and therefore the magnitude of $p_{\alpha}^{A}$ is not significant. The only thing which could possibly be significant in the logarithmic term, as in Part $I$, is the sign of the imaginary part of $p_{\alpha}^{A}$ which, as given in Appendix $I-A$, is positive. However, in the present case, we do not need to introduce a cut in the complex plane so that the limit $\varepsilon=0$ can be taken immediately. 
Following the method given in Papers $I$ and II and also part I, and using eq (9), we obtain the following expression for the stress $\tau_{i 2}$

$$
\underline{T}_{\infty 2}^{A}(\underline{X})=\underline{T}_{02}^{A}-\frac{1}{\pi} \sum_{\alpha} \underline{\sigma}^{A}(\alpha) \int_{-\infty}^{\infty} \frac{\underline{f}^{A}(t) d t}{z_{\alpha}^{A}-t+\iota \varepsilon},
$$

where the vector $\underline{T}_{0}^{A}$ is defined as

$$
\mathrm{T}_{02 \mathrm{i}}^{\mathrm{A}}=\mathrm{c}_{\mathrm{i} 233}^{\mathrm{A}} \eta \text {. }
$$

In a similar fashion we obtain the following expressions for the displacement field and the stress component $\tau_{i 2}$ in the solid $B$ in the LHP:

$$
\begin{gathered}
\underline{u}_{\infty}^{B}(\underline{X})=\underline{u}_{0}-\frac{1}{\pi} \sum_{\alpha} \underline{\bar{\gamma}}^{B}(\alpha) \int_{-\infty}^{\infty} \ln \left(\bar{z}_{\alpha}^{B}-t+\iota \varepsilon\right) \underline{f}^{B}(t) d t, \\
\underline{T}_{\infty 2}^{B}(\underline{X})=\underline{T}_{02}^{B}-\frac{1}{\pi} \sum_{\alpha} \underline{\sigma}^{B}(\alpha) \int_{-\infty}^{\infty} \frac{\underline{f}^{B}(t) d t}{\bar{z}_{\alpha}^{B}-t+\iota \varepsilon},
\end{gathered}
$$

where

$$
\underline{T}_{02 i}^{B}=c_{i 233}^{B} \eta
$$


Now we apply the boundary condition given by eq (5) on eqs (15) and (19). This gives the following relation between the two force functions:

$$
\underline{\underline{\gamma}}_{S}^{A} \underline{f}^{A}(t)=\underline{\underline{\gamma}}_{S}^{B} \underline{\underline{f}}^{B}(t) \text {. }
$$

For convenience, we write $\underline{f}^{A}(t)$ and $\underline{f}^{B}(t)$ in terms of another force function $\underline{f}_{0}(t)$ as

$$
\underline{f}^{A}(t)=\left[\underline{\gamma}_{s}^{A}\right]^{-1} \underline{f}_{0}(t)
$$

and

$$
\underline{f}^{B}(t)=\left[\underline{\bar{r}}_{s}^{B}\right]^{-1} \underline{f}_{0}(t) .
$$

We now substitute for $\underline{f}^{A}(t)$ and $\underline{f}^{B}(t)$ from eqs (23) and (24) in eqs (17) and (21) and apply the boundary condition given by eq (6). After rearranging the terms, we obtain the integral equation

$$
\frac{1}{\pi} \int_{-\infty}^{\infty} \frac{\underline{f}_{0}(t) d t}{x_{1}-t}=\underline{D} \underline{T}_{0},
$$

where

$$
\begin{gathered}
\underline{D}=\left\{\bar{\sigma}_{s}^{B}\left[\underline{\bar{\gamma}}_{s}^{\mathrm{B}}\right]^{-1}-\underline{\sigma}_{\mathrm{s}}^{\mathrm{A}}\left[\underline{\gamma}_{\mathrm{s}}^{\mathrm{A}}\right]^{-1}\right\}^{-1}, \\
\underline{\mathrm{T}}_{0}=\underline{\mathrm{T}}_{02}^{\mathrm{B}}-\underline{\mathrm{T}}_{02}^{\mathrm{A}},
\end{gathered}
$$


and the integral on the LHS of eq (25) is a principal value integral.

Equation (25) is in the standard form of a Hilbert transform. Its solution is given by

$$
\underline{f}_{0}\left(x_{1}\right)=\frac{1}{\pi} \underline{\mathrm{D}} \underline{\mathrm{T}}_{0} \int_{-\infty}^{\infty} \frac{\mathrm{dt}}{\mathrm{x}_{1}-t},
$$

where we have used the fact that the RHS of eq (25) is a constant. The integral on the RHS of eq (28) can be easily carried out. For this purpose, it would be convenient to evaluate the integral taking the upper and the lower limits to be $t_{\infty}$ and $-t_{\infty}$ respectively and then taking the limit $t_{\infty}=\infty$. The result is

$$
\underline{\mathrm{f}}_{0}\left(\mathrm{x}_{1}\right)=\iota \underline{\mathrm{D}} \underline{\mathrm{T}}_{0},
$$

where we have taken the following limiting values of the logarithmic function:

$$
\ln \left(x_{1}+t_{\infty}\right)=\ln \left(t_{\infty}\right)
$$

and

$$
\ln \left(x_{1}-t_{\infty}\right)=\ln \left(t_{\infty}\right)+\pi \iota
$$

in the limit $t_{\infty} \rightarrow \infty$.

From eqs (23), (24) and (29), we obtain the following solution for the two force functions:

$$
\underline{\underline{f}}^{\mathrm{A}}(t)=-\iota \quad \underline{\mathrm{M}} \underline{\underline{T}}_{0}
$$


and

$$
\underline{\mathrm{f}}^{\mathrm{B}}(t)=-\iota \underline{\mathrm{N}} \underline{\mathrm{T}}_{0},
$$

where the matrices $\underline{M}$ and $\underline{N}$ have been defined in eqs (A.11) and (A.12) in Part I.

We see from eqs (32) and (33) that the two force functions are constants. Using these results in eqs (15) and (19) gives the required expressions for the displacement field in the solid. The logarithmic integrals in eqs (15) and (19) can be easily evaluated. Thus we obtain the following result for the displacement field in the UHP and the LHP:

$$
\underline{u}_{\infty}^{A}(\underline{X})=\underline{u}_{0}-\sum_{\alpha} \underline{\gamma}^{A}(\alpha) \underline{M} \underline{T}_{0} z_{\alpha}^{A}
$$

and

$$
\underline{u}_{-\infty}^{B}(\underline{X})=\underline{u}_{0}-\sum_{\alpha} \underline{\underline{\gamma}}^{B}(\alpha) \underline{N}_{0} \bar{z}_{\alpha}^{B}
$$

where we have used eqs (30) and (31) and, as is usual in continuum mechanics, ignored the terms which correspond to the rigid body displacements in the solid.

For later use, the expressions for the stress components $\tau_{\text {il }}$ and $\tau_{i 2}$, which can be obtained from eqs (34) and (35) by using eqs (8) and (9), are given here.

$$
\begin{aligned}
& \underline{\mathrm{T}}_{\infty 1}^{\mathrm{A}}=\underline{\mathrm{T}}_{01}^{\mathrm{A}}-\underline{\Omega}_{\mathrm{S}}^{\mathrm{A}} \underline{\mathrm{M}} \mathrm{T}_{0}, \\
& \underline{\mathrm{T}}_{\infty 1}^{\mathrm{B}}=\underline{\mathrm{T}}_{01}^{\mathrm{B}}-\underline{\underline{\Omega}}_{\mathrm{S}}^{\mathrm{B}} \underline{\mathrm{T}}_{0},
\end{aligned}
$$




$$
\underline{T}_{-\infty 2}^{A}=\underline{T}_{02}^{A}-\underline{\sigma}_{S}^{A} \underline{M} T_{0}
$$

and

$$
\underline{T}_{\infty 2}^{\mathrm{B}}=\underline{\mathrm{T}}_{02}^{\mathrm{B}}-\underline{\sigma}_{\mathrm{S}}^{\mathrm{B}} \underline{\mathrm{N}} \mathrm{T}_{0},
$$

where the vectors $\mathrm{T}_{\infty 1}^{\mathrm{A}, \mathrm{B}}$ and $\mathrm{T}_{01}^{\mathrm{A}, \mathrm{B}}$ are defined in analogy with eqs (7) and (18), as

$$
\underline{T}_{-\infty}^{A}, B(\underline{X})=\tau_{i 1}^{A, B}
$$

and

$$
\mathrm{T}_{01 \mathrm{i}}^{\mathrm{A}, \mathrm{B}}=\mathrm{C}_{\mathrm{i}, \mathrm{B} 3}^{\mathrm{A}, \mathrm{B}} \eta
$$

Equations (34), (35), (38) and (39) satisfy the two boundary conditions given by eqs (5) and (6). Moreover, the second term in both the eqs (34) and (35) is a function of $x_{1}$ and $x_{2}$ and not of $x_{3}$, whereas the first term is a function of $x_{3}$ only. Hence the value of the strain component $\varepsilon_{33}$ will be $\eta$ as prescribed by eq (4). Equations (34) and (35) are, therefore, the desired solution for the displacement in an infinite composite solid. It may be noted that $\tau_{i 2}$ is constant in the solid.

3. Displacement and the Stress Field in a Composite Solid Containing a Free Surface

In this section we shall apply the results obtained in the last section to calculate the displacement field and the stress distribution in composite containing a free surface. The model solid and the coordinate axes are shown in figure I-1 of Part I. Mathematically, the difference between the calculations of Part I and the present calculations arises because in Part I a line load 
was specified at a given point whereas in the present case the strain $\varepsilon_{33}$ is specified.

We start by writing the displacement fields in $A$ and $B$ in the following form, which is analogous to that given in eq ( 3 ).

$$
u_{i}^{A}(\underline{X})=u_{\infty i}^{A}(\underline{X})+u_{s i}^{A}(\underline{x})
$$

and

$$
u_{i}^{B}(\underline{X})=u_{\infty i}^{B}(\underline{X})+u_{s i}^{B}(\underline{x})
$$

where $\underline{u}_{-\infty}^{A, B}(\underline{X})$ are the displacement field vectors in an infinite composite with no free surfaces as given by eqs (25) and (36), and $\underline{u}_{s}^{A, B}(\underline{x})$ are functions of only $x_{1}$ and $x_{2}$, and not of $x_{3}$.

In view of the remark made at the end of the preceding section, the strain tensor calculated from displacement field as given by eqs (42) and (43) will satisfy eq (4). Our task, therefore, is to determine $\underline{u}_{s}^{A}, B(\underline{x})$ such that eqs $(42)$ and (43) are solutions of the elastic equilibrium equations [eq ( 1 ) with appropriate superscripts] in the UHP and LHP respectively and satisfy the boundary conditions,

$$
\begin{gathered}
u^{A}{ }_{i}\left[x_{1}, 0\right]=u_{i}^{B}\left[x_{1}, 0\right] \quad\left(-\infty \leq x_{1} \leq \infty\right), \\
\tau^{A}{ }_{i 2}\left[x_{1}, 0\right]=\tau_{i 1}^{B}\left[x_{1}, 0\right] \quad\left(-\infty \leq x_{1} \leq \infty\right), \\
\tau^{A}{ }_{i 1}\left[0, x_{2}\right]=0 \quad\left(0 \leq x_{2} \leq \infty\right),
\end{gathered}
$$

and

$$
\tau^{B}{ }_{i 1}\left[0, x_{2}\right]=0 \quad\left(0 \geq x_{2} \geq-\infty\right)
$$


We can determine $\underline{u}_{s}^{A, B}(\underline{x})$ by using the plane-strain Green's function as quoted in Appendix I-A. This Green's function is a solution of eq (1) and satisfies the boundary conditions given by eqs (44) and (45). In order to satisfy the boundary condition given by eqs (46) and (47), we shall use the same technique as used in Part $I$.

3A. Solution in Terms of an Integral Representation

As in Part I, we apply a hypothetical distribution of line forces just outside the free surface denoted by $\underline{F}^{A}(t)$ in the UHP and $\underline{F}^{B}(t)$ in the LHP. As shown in figure II-2, these forces are applied just outside the free surface at continuous set of points $x_{1}=-\varepsilon$ and $x_{2}=t$, where $\varepsilon$ is a small positive number which is taken to be zero in the limit. The range of the real variable $t$ is $(0 \leq t \leq \infty)$ for $\underline{F}^{A}(t)$ and $(-\infty \leq t \leq 0)$ for $\underline{F}^{B}(t)$ in the LHP.

Thus, using the definition of the Green's function and as given by eq (2), we can write the following expressions for $u_{s}^{A, B}(\underline{x})$ in UHP and LHP

$$
u_{s i}^{A}(\underline{x})=\int_{0}^{\infty} \underline{G}_{0}^{A A}(\underline{x} ; t) \underline{F}^{A}(t) d t+\int_{-\infty}^{0} G_{0}^{A B}(\underline{x} ; t) \underline{F}^{B}(t) d t
$$

and

$$
u_{s i}^{B}(\underline{x})=\int_{0}^{\infty} \underline{G}_{0}^{B A}(\underline{x} ; t) \underline{F}^{A}(t) d t+\int_{-\infty}^{0} \underline{G}_{0}^{B B}(\underline{x} ; t) \underline{F}^{B}(t) d t,
$$

where $G_{0}$ denotes the Green's function for an infinite bimaterial composite which is given by eqs (A.1) -- (A.4) in Appendix I-A. Using these equations and eqs (117) and (118) of Part $I$, we obtain the following expressions for the displacement field from eqs $(42),(43),(48)$ and $(49)$ : 


$$
\begin{aligned}
& \underline{u}^{A}(\underline{x})=\underline{u}_{\infty}^{A}(X)+\iota \sum_{\alpha}\left[\underline{\gamma}^{A}(\alpha) \underline{U}^{A}\left(\frac{z_{\alpha}^{A} \varepsilon}{p_{\alpha}^{A}}\right)+\underline{\gamma}^{-A}(\alpha) \underline{U}^{A}\left(\frac{\bar{z}_{\alpha}^{+} \varepsilon}{\bar{p}_{\alpha}^{A}}\right)\right] \\
& +\iota \sum_{\alpha \beta}\left[\underline{E}_{\mathrm{AA}}(\alpha, \beta) \underline{U}^{\mathrm{A}}\left(\frac{\mathrm{z}_{\alpha}^{\mathrm{A}}}{\bar{p}_{\beta}^{\mathrm{A}}}\right)+\bar{E}_{\mathrm{AA}}(\alpha, \beta) \underline{U}^{\mathrm{A}}\left(\frac{\bar{z}_{\alpha}^{\mathrm{A}}}{\mathrm{p}_{\beta}^{\mathrm{A}}}\right)\right] \\
& -\iota \sum_{\alpha \beta}\left[\underline{E}_{A B}(\alpha, \beta) \underline{U}^{B}\left(\frac{z_{\alpha}^{A}}{p_{\beta}^{B}}\right)+\bar{E}_{A B}(\alpha, \beta) \underline{U}^{B}\left(\frac{\bar{z}_{\alpha}^{A}}{\bar{p}_{\beta}^{B}}\right)\right]
\end{aligned}
$$$$
\text { (for } \left.0 \leq x_{2} \leq \infty\right)
$$

and

$$
\begin{aligned}
& \underline{u}^{B}(\underline{x})=\underline{u}_{-\infty}^{B}(x)+\iota \sum_{\alpha \beta}\left[\underline{E}_{B A}(\alpha, \beta) \underline{U}^{A}\left(\frac{\bar{z}_{\alpha}^{B}}{\bar{p}_{\beta}^{A}}\right)+\underline{E}_{B A}(\alpha, \beta) \underline{U}^{A}\left(\frac{z_{\alpha}^{B}}{p_{\beta}^{A}}\right)\right] \\
& +\iota \sum_{\alpha}\left[\underline{\gamma}^{\mathrm{B}}(\alpha) \underline{\mathrm{U}}^{\mathrm{B}}\left(\frac{\mathrm{z}_{\alpha}^{\mathrm{B}}+\varepsilon}{\mathrm{p}_{\alpha}^{\mathrm{B}}}\right)+\underline{\bar{\gamma}}^{\mathrm{B}}(\alpha) \underline{\mathrm{U}}^{\mathrm{B}}\left(\frac{\overline{\mathrm{z}}_{\alpha}^{\mathrm{B}}+\varepsilon}{\overline{\mathrm{p}}_{\alpha}^{\mathrm{B}}}\right)\right] \\
& -\iota \sum_{\alpha \beta}\left[\underline{E}_{\mathrm{BB}}(\alpha, \beta) \underline{\mathrm{U}}^{\mathrm{B}}\left(\frac{\bar{z}_{\alpha}^{\mathrm{B}}}{\mathrm{p}_{\beta}^{\mathrm{B}}}\right)+\overline{\underline{E}}_{\mathrm{BB}}(\alpha, \beta) \underline{H}^{\mathrm{B}}\left(\frac{z_{\alpha}^{\mathrm{B}}}{\bar{p}_{\beta}^{\mathrm{B}}}\right)\right] \\
& \text { (for }-\infty \leq x_{2} \leq 0 \text { ), }
\end{aligned}
$$

where, as in Part $I$, the $U$ - functions are defined as the indefinite integrals,

$$
\underline{U}^{A}(z)=\int H^{A}(z) d z
$$




$$
\begin{gathered}
\underline{\mathrm{U}}^{\mathrm{B}}(\mathrm{z})=\int \mathrm{H}^{\mathrm{B}}(\mathrm{z}) \mathrm{dz}, \\
\underline{\mathrm{H}}^{\mathrm{A}}(\mathrm{z})=\frac{1}{2 \pi \iota} \int_{0}^{\infty} \frac{\mathrm{F}^{\mathrm{A}}(t)}{\mathrm{t}-\mathrm{z}} d t
\end{gathered}
$$

and

$$
\underline{H}^{B}(z)=\frac{1}{2 \pi \iota} \int_{-\infty}^{0} \frac{F^{B}(t)}{t-z} d t,
$$

and the other symbols have been defined in Part I.

For later use, we also write below the stress components $\tau_{\text {i2 }}$ and $\tau_{i 1}$, which can be easily obtained from the displacement field given in eqs (50) and (51).

$$
\underline{T}_{2}^{\mathrm{A}}(\underline{x})=\underline{T}_{2 \infty}^{\mathrm{A}}
$$

$$
\begin{aligned}
& +\iota \sum_{\alpha}\left[\left\{\underline{\sigma}^{\mathrm{A}}(\alpha) / \mathrm{p}_{\alpha}^{\mathrm{A}}\right\} \underline{\mathrm{H}}^{\mathrm{A}}\left(\frac{\mathrm{z}_{\alpha}^{\mathrm{A}}+\varepsilon}{\mathrm{p}_{\alpha}^{\mathrm{A}}}\right)+\left\{\bar{\sigma}^{\mathrm{A}}(\alpha) / \overline{\mathrm{p}}_{\alpha}^{\mathrm{A}}\right\} \underline{\mathrm{H}}^{\mathrm{A}}\left(\frac{\overline{\mathrm{z}}_{\alpha}^{\mathrm{A}}+\varepsilon}{\overline{\mathrm{p}}_{\alpha}^{\mathrm{A}}}\right)\right] \\
& +\iota \sum_{\alpha \beta}\left[\underline{\mathrm{D}}_{\mathrm{AA}}(\alpha, \beta) \underline{H}^{\mathrm{A}}\left(\frac{\mathrm{z}_{\alpha}^{\mathrm{A}}}{\overline{\mathrm{p}}_{\beta}^{\mathrm{A}}}\right)+\overline{\mathrm{D}}_{\mathrm{AA}}(\alpha, \beta) \underline{H}^{\mathrm{A}}\left(\frac{\overline{\mathrm{z}}_{\alpha}^{\mathrm{A}}}{\mathrm{p}_{\beta}^{\mathrm{A}}}\right)\right] \\
& -\iota \sum_{\alpha \beta}\left[\underline{\mathrm{D}}_{\mathrm{AB}}(\alpha, \beta) \underline{H}^{\mathrm{B}}\left(\frac{\mathrm{z}_{\alpha}^{\mathrm{A}}}{\mathrm{p}_{\beta}^{\mathrm{B}}}\right)+\overline{\mathrm{D}}_{\mathrm{AB}}(\alpha, \beta) \underline{H}^{\mathrm{B}}\left(\frac{\overline{\mathrm{z}}_{\alpha}^{\mathrm{A}}}{\overline{\mathrm{p}}_{\beta}^{\mathrm{B}}}\right)\right],
\end{aligned}
$$




$$
\begin{aligned}
& \underline{T}_{2}^{B}(\underline{x})=\underline{T}_{2 \infty}^{B} \\
& +\iota \sum_{\alpha \beta}\left[\underline{D}_{B A}(\alpha, \beta) \underline{H}^{A}\left(\frac{\bar{z}_{\alpha}^{B}}{\bar{p}_{\beta}^{A}}\right)+\underline{D}_{B A}(\alpha, \beta) \underline{H}^{A}\left(\frac{z_{\alpha}^{B}}{p_{\beta}^{A}}\right)\right] \\
& +\iota \sum_{\alpha}\left[\left\{\underline{\sigma}^{\mathrm{B}}(\alpha) / \mathrm{p}_{\alpha}^{\mathrm{B}}\right\} \underline{\mathrm{H}}^{\mathrm{B}}\left(\frac{\mathrm{z}_{\alpha}^{\mathrm{B}} \boldsymbol{\varepsilon}}{\mathrm{p}_{\alpha}^{\mathrm{B}}}\right)+\left\{\underline{\bar{\sigma}}^{\mathrm{B}}(\alpha) / \overline{\mathrm{p}}_{\alpha}^{\mathrm{B}}\right\} \underline{\mathrm{H}}^{\mathrm{B}}\left(\frac{\overline{\mathrm{z}}_{\alpha}^{\mathrm{B}} \boldsymbol{\varepsilon}}{\overline{\mathrm{p}}_{\alpha}^{\mathrm{B}}}\right)\right] \\
& -\iota \sum_{\alpha \beta}\left[\underline{D}_{\mathrm{BB}}(\alpha, \beta) \underline{H}^{\mathrm{B}}\left(\frac{\bar{z}_{\alpha}^{\mathrm{B}}}{\mathrm{p}_{\beta}^{\mathrm{B}}}\right)+\overline{\mathrm{D}}_{\mathrm{BB}}(\alpha, \beta) \underline{H}^{\mathrm{B}}\left(\frac{z_{\alpha}^{\mathrm{B}}}{\bar{p}_{\beta}^{\mathrm{B}}}\right)\right], \\
& \underline{\mathrm{T}}_{1}^{\mathrm{A}}(\underline{\underline{x}})=\mathrm{T}_{\infty 1}^{\mathrm{A}}+\iota \sum_{\alpha}\left[\underline{\mathrm{J}}^{\mathrm{A}}(\alpha) \underline{\mathrm{H}}^{\mathrm{A}}\left(\frac{\mathrm{z}_{\alpha}^{\mathrm{A}}+\varepsilon}{\mathrm{p}_{\alpha}^{\mathrm{A}}}\right)+\underline{\bar{J}}^{\mathrm{A}}(\alpha) \underline{\mathrm{H}}^{\mathrm{A}}\left(\frac{\overline{\mathrm{z}}_{\alpha}^{\mathrm{A}}+\varepsilon}{\overline{\mathrm{p}}_{\alpha}^{\mathrm{A}}}\right)\right] \\
& +\iota \sum_{\alpha \beta}\left[\underline{\mathrm{K}}_{\mathrm{AA}}(\alpha, \beta) \underline{\mathrm{H}}^{\mathrm{A}}\left(\frac{\mathrm{z}_{\alpha}^{\mathrm{A}}}{\overline{\mathrm{p}}_{\beta}^{\mathrm{A}}}\right)+\overline{\mathrm{K}}_{\mathrm{AA}}(\alpha, \beta) \underline{H}^{\mathrm{A}}\left(\frac{\overline{\mathrm{z}}_{\alpha}^{\mathrm{A}}}{\mathrm{p}_{\beta}^{\mathrm{A}}}\right)\right] \\
& -\iota \sum_{\alpha \beta}\left[\underline{\mathrm{K}}_{\mathrm{AB}}(\alpha, \beta) \underline{H}^{\mathrm{B}}\left(\frac{\mathrm{z}_{\alpha}^{\mathrm{A}}}{\mathrm{p}_{\beta}^{\mathrm{B}}}\right)+\overline{\mathrm{K}}_{\mathrm{AB}}(\alpha, \beta) \underline{\mathrm{H}}^{\mathrm{B}}\left(\frac{\overline{\mathrm{z}}_{\alpha}^{\mathrm{A}}}{\overline{\mathrm{p}}_{\beta}^{\mathrm{B}}}\right)\right]
\end{aligned}
$$

and

$\underline{\mathrm{T}}_{1}^{\mathrm{B}}(\underline{x})=\mathrm{T}_{\infty 1}^{\mathrm{B}}+\iota \sum_{\alpha \beta}\left[\underline{\mathrm{K}}_{\mathrm{BA}}(\alpha, \beta) \underline{\mathrm{H}}^{\mathrm{A}}\left(\frac{\bar{z}_{\alpha}^{\mathrm{B}}}{\overline{\mathrm{p}}_{\beta}^{\mathrm{A}}}\right)+\underline{\overline{\mathrm{K}}}_{\mathrm{BA}}(\alpha, \beta) \underline{\mathrm{H}}^{\mathrm{A}}\left(\frac{\mathrm{z}_{\alpha}^{\mathrm{B}}}{\mathrm{p}_{\beta}^{\mathrm{A}}}\right)\right]$ 


$$
\begin{aligned}
& +\iota \sum_{\alpha}\left[\underline{J}^{\mathrm{B}}(\alpha) \underline{\mathrm{H}}^{\mathrm{B}}\left(\frac{\mathrm{z}_{\alpha}^{\mathrm{B}} \varepsilon}{\mathrm{p}_{\alpha}^{\mathrm{B}}}\right)+\underline{\bar{J}}^{\mathrm{B}}(\alpha) \underline{\mathrm{H}}^{\mathrm{B}}\left(\frac{\bar{z}_{\alpha}^{\mathrm{B}}+\varepsilon}{\overline{\mathrm{p}}_{\alpha}^{\mathrm{B}}}\right)\right] \\
& -\iota \sum_{\alpha \beta}\left[\underline{\mathrm{K}}_{\mathrm{BB}}(\alpha, \beta) \underline{\mathrm{H}}^{\mathrm{B}}\left(\frac{\overline{\mathrm{z}}_{\alpha}^{\mathrm{B}}}{\mathrm{p}_{\beta}^{\mathrm{B}}}\right)+\overline{\mathrm{K}}_{\mathrm{BB}}(\alpha, \beta) \underline{\mathrm{H}}^{\mathrm{B}}\left(\frac{\mathrm{z}_{\alpha}^{\mathrm{B}}}{\overline{\mathrm{p}}_{\beta}^{\mathrm{B}}}\right)\right],
\end{aligned}
$$

where the vectors $\underline{T}_{\infty 1}$ and $\underline{T}_{\infty 2}$ are given by eqs $(36)-(39)$ and, in an analogous manner, we have defined the vectors $\underline{T}_{1}(\underline{x})$ and $\underline{T}_{2}(\underline{x})$ such that their $i^{\text {th }}$ components are equal to, respectively, $\tau_{i 1}(\underline{x})$ and $\tau_{i 2}(\underline{x})$.

As mentioned earlier, eqs (50) and (51) will satisfy eq (1) everywhere in the region of solution and also the continuity conditions at the interface as given by eqs (44) and (45) for all values of $\underline{F}^{A}(t)$ and $\underline{F}^{B}(t)$. This is because the forces have been applied outside the region of solution and the Green's function used in writing eqs (50) and (51) satisfies eqs (44) and (45). As in Part $I$, we have to determine these two force functions so that the free surface boundary conditions as given by eqs (46) and (47) are also satisfied. In addition, the force functions have to satisfy the following condition required for the displacements to be single-valued:

$$
\int_{0}^{\infty} \underline{F}^{A}(t) d t+\int_{-\infty}^{0} \underline{F}^{B}(t) d t=0 .
$$

The $\mathrm{H}$ - functions are determined in the same way as in Part I. We write these in the form,

$$
\underline{\mathrm{H}}^{\mathrm{A}}(\mathrm{z})=\int_{-\infty}^{\infty} \underline{\mathrm{v}}^{\mathrm{A}}(q) \mathrm{z}^{l q-0.5} \mathrm{dq}
$$


and

$$
\underline{H}^{B}(z)=\int_{-\infty}^{\infty} \underline{v}^{B}(q) z^{l q-0.5} d q \text {, }
$$

where $\underline{v}^{A}(q)$ and $\underline{v}^{B}(q)$ are the unknown functions of the real variable $q$, which have to be determined.

Following exactly the same procedure as given in Part I and using the same block representation, we obtain the following result for the vectors $\underline{v}^{A}(q)$ and $\underline{v}^{B}(q)$,

$$
\underline{M}(q) \underline{V}(q)=\underline{N}(q) \text {, }
$$

where $M(q)$ is exactly the same as given by eqs $(38)-(41)$ of Part I and the block elements of $\underline{N}(q)$ are

and

$$
N_{A}(q)=-\frac{T_{-\infty 1}^{A}}{2 \pi \iota} \int_{0}^{\infty} y^{-\iota q-0.5} d y
$$

$$
N_{B}(q)=-\frac{\underline{T}_{-\infty 1}^{B}}{2 \pi \iota} \int_{-\infty}^{0}|y|^{-\iota q-0.5} d y .
$$

The integrals in eqs (64) and (65) can be evaluated in an elementary manner. However, in order to be able to handle the apparent divergence at $\infty$, we evaluate the integral in eq (64) between the limits 0 and $y_{\infty}$ and that in eq (65) between $-y_{\infty}$ and 0 , and in the end take the limit $y_{\infty}=\infty$. Thus we obtain the following expressions for the block elements of $\mathrm{N}$ : 


$$
N_{A}(q)=t_{\infty}^{A}(q) /(-\iota q+0.5)
$$

and

$$
\underline{N}_{B}(q)=t_{\infty}^{B}(q) /(-\llcorner q+0.5),
$$

where

$$
\underline{t}_{\infty}^{A}(q)=-\frac{\underline{T}_{\infty 1}^{A}}{2 \pi \iota} y_{\infty}^{-\iota q+0.5}
$$

and

$$
\underline{t}_{\infty}^{B}(q)=-\frac{\underline{T}_{\infty 1}^{B}}{2 \pi \iota} y_{\infty}^{-\iota q+0.5} .
$$

The transform of a constant in q-space as given by eqs (68) and (69) is singular in the limit $Y_{\infty}=\infty$. However, as shown in Appendix II-A, this singularity cancels out when the inverse transform is taken.

The solution of the matrix equation (63) can be easily written as

$$
\underline{V}(q)=[\underline{M}(q)]^{-1} \underline{t}_{\infty}(q) /(-\iota q+0 \cdot 5) \text {. }
$$

For later use, we write the following block structure of eqs (63) and $(70)$ as in Part I:

$$
\left(\begin{array}{ll}
\underline{M}_{\mathrm{AA}} & \underline{\mathrm{M}}_{\mathrm{AB}} \\
\underline{\mathrm{M}}_{\mathrm{BA}} & \underline{-}_{\mathrm{BB}}
\end{array}\right)\left(\begin{array}{l}
\underline{v}^{\mathrm{A}}(q) \\
\underline{v}^{B}(q)
\end{array}\right)=\frac{1}{(-\iota q+0.5)}\left(\begin{array}{c}
\underline{t}_{-}^{\mathrm{A}} \\
\underline{t}_{\infty}^{\mathrm{B}}
\end{array}\right)
$$


and

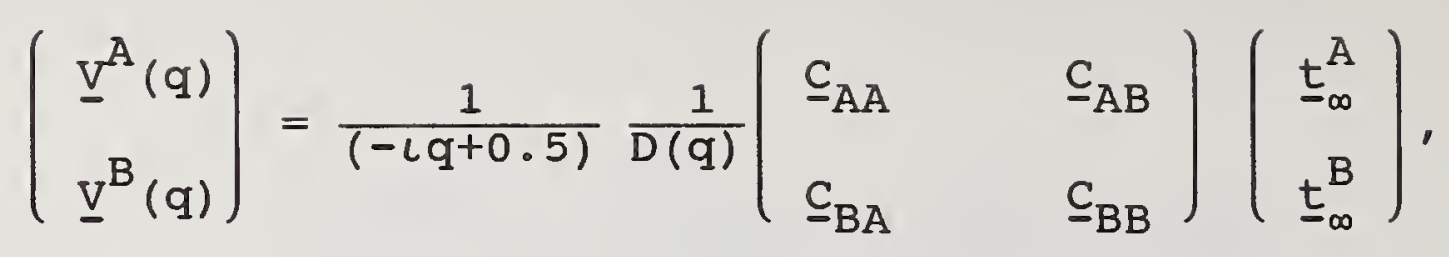

where $\underline{C}$ is the matrix of cofactors of $\underline{M}$.

As remarked in Part $I$, the representation of $\underline{V}(q)$ in terms of the cofactor matrix as given by eq (72) is useful mainly for the purpose of a qualitative analysis of the nature of $\underline{H}(z)$. For numerical calculations, it is better to use eq (70), which involves only the inversion of a $6 \times 6$ matrix.

Equation (70) gives the particular solution of the Hilbert's equation. The general solution is obtained by adding the homogeneous and the particular solutions and is written as

$$
\underline{H}(z)=\left(\begin{array}{c}
\underline{H}_{p}^{A}(z) \\
\underline{H}_{p}^{B}(z)
\end{array}\right)+\left(\begin{array}{c}
\underline{H}_{0}^{A}(z) \\
\underline{H}_{0}^{B}(z)
\end{array}\right),
$$

where the subscript $\mathrm{p}$ denotes the particular solution which is given by eqs (61) and (62) along with eq (70). The homogeneous part of the solution in eq (73), labelled with the subscript 0 , is

$$
\underline{H}_{0}(z)=\sum_{r} \underline{h}\left(Q_{r}\right) z^{\iota Q_{r}-0.5},
$$

where the vector $\underline{h}$ is a solution of the matrix equation,

$$
\underline{M}\left(Q_{r}\right) \underline{h}\left(Q_{r}\right)=0
$$


and $Q_{r}$ is a root of the determinantal equation,

$$
D\left(Q_{r}\right)=0
$$

One of the components of the vector $\underline{h}$ for each $Q_{r}$ will be arbitrary. It has to be chosen so that eq $(60)$ is satisfied. These constants can also be chosen to satisfy any other boundary condition on the stress field, such as those which may be required at the outer surfaces in a finite solid.

3B. Series Representation for the Particular Solution

In this subsection, we shall obtain the series representation for the particular solution for $\underline{H}(z)$. It may be remarked that for an actual calculation of $\underline{H}(z)$, it would be generally more convenient to evaluate numerically the integral over $q$ in eqs (61) and (62). The series representation for $\underline{H}(z)$ may not give numerically accurate values of $\underline{H}(z)$ except for limiting values of mod $(z)$, because the convergence of the series is not guaranteed.

However, the series representation for $\underline{H}(z)$ serves two useful purposes: (i) it can be used as a numerical check on the direct calculation of $\underline{H}(z)$ obtained by numerical integration in eqs (61) and (62), and (ii) it helps in identifying the singularities in $\underline{H}(z)$ at $\bmod (z)=0$, which provides a better physical understanding of the nature of the stress distribution in the solid. Moreover, as mentioned in section 1 , a knowledge of the singularities in $\underline{H}(z)$ is essential for a proper numerical modelling of the solid by using finite element or other such techniques.

First, we shall consider $\underline{H}^{\mathrm{A}}(\mathrm{z})$. We see from eqs (61) and (72) that the particular solution for $\underline{H}^{A}(z)$ can be written as 


$$
\underline{H}^{A}(z)=\int_{-\infty}^{\infty} \frac{\underline{p}^{A}(q)\left[z / y_{\infty}\right]^{i q-0.5} d q}{(-\iota q+0.5) D(q)},
$$

where

$$
\underline{\mathrm{P}}^{A}(q)=-\frac{1}{2 \pi \iota}\left[\underline{\mathrm{C}}_{\mathrm{AA}}(q) \underline{\mathrm{T}}_{-\infty 1}^{\mathrm{A}}+\underline{\mathrm{C}}_{\mathrm{AB}}(q) \underline{T}_{-\infty 1}^{\mathrm{A}}\right],
$$

with a similar expression for $\underline{H}^{B}(z)$.

We evaluate the integral in eq (77) by following the same procedure as used in section 3 in part I. Comparing eq (77) with eq (C.16) of Part $I$, we find that the only poles which can contribute to the integral in eq (77) are those arising from the zeroes of $D(q)$ and the single pole at $q_{-1}=-\iota / 2$. The set-I poles in eq (C.16) of Part I arising from the function $E(q)$ are not present in eq (77) except for the single pole at $q=q_{-1}$. Moreover, in the two examples discussed in section 4 and section 5 , we shall see that $D\left(q_{-1}\right)$ is also zero. Hence, the pole at $q=$ $q_{-1}$ is a second-order pole in the lower half of the complex plane.

Since $y_{\infty}$ approaches $\infty$ in the limit, we note that $\bmod \left(z / y_{\infty}\right)$ in eq (77) will approach 0 . Thus, as indicated in section $3 \mathrm{~B}$ and Appendix I-C in Part $I$, we have to choose the contour in the LHP for evaluating the integral over $q$ in eq (77). Thus, by using eqs (C.34) and (73) of Part $I$, we obtain the following series representation for $\underline{\mathrm{H}}(\mathrm{z})$ :

$$
\underline{H}(z)=-\sum_{Y} \underline{\Psi}\left(Q_{r}, \varepsilon\right) \xi\left(\iota Q_{r}, \varepsilon\right) z^{\iota Q_{r}-0.5+\varepsilon}-\underline{R}_{H}(z),
$$

where $Q_{r}$ is a root of eq (76) with the constraint, 


$$
\operatorname{Im}\left(Q_{r}\right)<0,
$$

and the other quantities in eq (79) have been defined in Part I. In eq (79) and in what follows in this section, $z$ is expressed in units of $y_{\infty}$ for notational convenience. In actual numerical calculations $y_{\infty}$ serves as a scale factor.

In analogy with eq (103) of Part $I$, we write eq (79) in the form,

$$
\underline{H}_{p}(z)=\sum_{r} \underline{V}_{p}\left(Q_{r}\right)[z]{ }^{\left(k_{r}-0.5\right)} \exp \left[\lg _{r} \ln (z)\right]+\underline{R}_{H}(z),
$$

where $g_{r}$ and $-k_{r}$ are the real and imaginary parts of $Q_{r}$ '

$$
Q_{r}=g_{r}-\iota k_{r}
$$

and

$$
\underline{\mathrm{V}}_{\mathrm{p}}\left(Q_{r}\right)=-\underline{\Psi}\left(Q_{r}\right) \xi\left(\iota Q_{r}\right)
$$

We see from eq (81) that $\underline{H}(z)$ will have the same kind of singularity and the logarithmic oscillations as discussed in section $3 B$ of Part $I$. The singularity will exist only if a $Q_{r}$ exists such that its imaginary part satisfies the constraint that

$$
0<k_{r}<0.5 \text {. }
$$

Similarly, the logarithmic oscillatory factor will exist only if the real part of $Q_{r}$ is nonzero.

In eq (81), the first term contains sum over only those values of $\mathrm{Q}_{r}$ which are not higher order poles. The contribution of 
higher order poles is included in $\underline{R}_{H}(z)$. In particular, the contribution $\underline{R}_{\mathrm{HO}}(z)$ of the second-order pole at

$$
q_{-1}=Q_{-1}=-\iota / 2
$$

to $\underline{R}_{\mathrm{H}}(z)$ has been given below. Following the method leading to eq (104) in Part $I$, we obtain

$$
\underline{R}_{H O}(z)=\frac{l}{D^{\prime}\left(q_{-1}\right)}\left[\underline{P}^{\prime}\left(q_{-1}\right)+\iota \underline{P}\left(q_{-1}\right) \ln (z)\right] \text {. }
$$

Equation (86) shows the $\ln (z)$ behavior of $\mathrm{H}(\underline{z})$ in agreement with some of the earlier work (see, for example, [10] and other references given there). Further, if the root $Q_{r}$ is degenerate, we shall get logarithmic terms with higher powers. For example, if for some $Q_{r} \neq-l / 2$ which satisfies the constraint given by eq (84), $D\left(Q_{r}\right)$ has a factor $\left(q-Q_{r}\right)^{\eta}$, then by using the cauchy's theorem we can easily show that $R_{H}(z)$ will have the form,

$$
\mathrm{R}_{\mathrm{H}}(z) \approx[z] \mathrm{k}_{\mathrm{r \eta}}^{-0.5)}[\ln (z)]^{\eta-1} \exp \left[\lg _{\mathrm{r \eta}} \ln (z)\right]
$$

where $Q_{r}$ is written in terms of its real and imaginary parts as

$$
e_{r}=g_{r \eta}-\iota k_{r \eta}
$$

with the constraint

$$
\mathrm{k}_{r \eta}>0
$$

The subscript $\eta$ in eq (88) identifies the particular value of $Q_{r}$ as a $\eta$--fold degenerate root. 
3C. Force Functions and the Homogeneous Solution

In this subsection we calculate the force functions and their integral by using the same technique as in Part $I$. By using the Plemelj relation as given by eq $(B .42)$ of Part $I$, we obtain the following expression for the two force functions:

$$
I_{F}^{A}(y)=\int_{-\infty}^{\infty} \frac{E(q) \underline{P}^{A}(q) d q}{(\iota q-0.5) D(q)}\left(y / y_{\infty}\right) i q-0.5
$$

and

$$
I_{F}^{B}(y)=\int_{-\infty}^{\infty} \frac{\exp (\pi q) E(q) \underline{P}^{B}(q) d q}{(\iota q-0.5) D(q)}\left|y / y_{\infty}\right|^{i q-0.5}
$$

where $\underline{P}^{A}(q)$ has been defined in eq $(78)$, and

$$
\underline{P}^{B}(q)=-\frac{1}{2 \pi \iota}\left[\underline{C}_{B A}(q) \underline{T}_{\infty 1}^{A}+\underline{C}_{B B}(q) \underline{T}_{\infty 1}^{B}\right]
$$

The integrals over $q$ in eqs (89) and (90) can be evaluated by using the same method as given in Appendix I-C of Part I for calculating the integral in eq (C.31). Unlike eq (C.31) in Part I, the integrands in eqs $(89)$ and $(90)$ do not have the factor $E(q)$ in the denominator but have the factor $(-\iota q+0.5)$. The singularity arising from this factor at $q=-\imath / 2$ cancels out with the zero in $E(q)$ in the numerator. The integrands, therefore, have only a simple pole at $q=-\iota / 2$ which is a zero of $D(q)$.

We need only to calculate the total integral of the force functions in order to satisfy eq (60). The total integral of the 
force functions has also been evaluated in Appendix I-C of Part I. From eq (C.33) of Part I and eqs (88) and (89), we obtain the following expression for the integral of the force in UHP:

$$
\begin{aligned}
I_{T}^{A} & =\int_{0}^{Y_{\infty}} I_{F}^{A}(y) d y \\
& =\int_{-\infty}^{\infty} \frac{Y_{\infty} E(q) \underline{P}^{A}(q) d q}{D(q)(i q+0.5)(-i q+0.5)}\left[Y_{\infty} / Y_{\infty}\right]^{\iota q+0.5}
\end{aligned}
$$

We take the limit as $Y_{\infty}>Y_{\infty} \rightarrow \infty$. As explained in the Appendix I-C of Part I, only the poles in the LHP contribute to the $q$-integral at the lower limit of $Y$, and their contribution is zero. At the upper limit of $y$, the q-integral is shown in eq (93). In this case the poles in the UHP will contribute in the limit $\mathrm{Y}_{\infty}=\infty$.

First we consider the pole at $q=\iota / 2$. In contrast to eq (C.33) of Part $I$, the integrand in eq (93) has the $E(q)$ term in the numerator, which cancels the singularity arising from the factor $(-(q+0.5)$ in the denominator. However, as given in eq (C.25) of Part $I, D(q)$ is also zero at $q=\iota / 2$. Thus the integrand in eq (93) has a simple pole at $q=\iota / 2$ which arises from the zero of $D(q)$. The contribution of this pole is given by

$$
I_{\mathrm{TO}}^{\mathrm{A}} \approx 4 \pi^{2} Y_{\infty} \underline{\mathrm{P}}^{\mathrm{A}}\left(q_{0}\right) / \mathrm{D}^{\prime}\left(q_{0}\right)
$$

where the prime denotes differentiation with respect to $q$ and

$$
q_{0}=\iota / 2
$$


Similarly we obtain

$$
I_{\text {TO }}^{B} \approx 4 \pi^{2} Y_{\infty} \underline{P}^{B}\left(q_{0}\right) / D^{\prime}\left(q_{0}\right)
$$

Using eqs (78), (92), (94), (96) and eqs (93) and (94) of Part I, we see that the contribution of the pole at $q_{0}$ to the total force integral is zero.

Further, as in part $I$, we note from eq (93) that the contribution of a pole for which $\operatorname{Im}\left(Q_{r}\right)>0.5$, will be zero in the limit $Y_{\infty}=\infty$, because it would lead to a negative power of $Y_{\infty}$. Thus, we see that only those poles will contribute to the integral which satisfy the following constraint:

$$
0<\operatorname{Im}\left(Q_{r}\right)<0.5
$$

This leads to the following for the total force integral:

$$
\begin{gathered}
I_{t}=2 \pi \iota \sum_{Q_{r} \neq \iota / 2} \frac{E\left(Q_{r}\right) y_{\infty}\left(Y_{\infty} / Y_{\infty}\right)^{\iota Q_{r}+0.5}}{D^{\prime}\left(Q_{r}\right)\left(Q_{r}^{2}+1 / 4\right)} \\
{\left[\underline{P}^{A}\left(Q_{r}\right)-\iota \exp \left(\pi Q_{r}\right) \underline{P}^{B}\left(Q_{r}\right)\right],}
\end{gathered}
$$

where $Q_{r}$ is subject to the constraint given by eq (97). If a $Q_{r}$ which satisfies this constraint does not exist, then $I_{t}$ is 0 .

As remarked in Part $I$, the apparent singularities in $I_{t}$ are not relevant. When $I_{t}$ is not zero, we have to include additional force terms corresponding to the solution of the homogeneous equation. The H-functions as obtained by these terms are 
and

$$
\underline{H}_{0}^{A}(z)=\sum_{Q_{r} \neq \iota / 2} v_{0}^{A}\left(Q_{r}\right) z^{\iota Q_{r}-0.5}
$$

$$
\underline{H}_{0}^{B}(z)=\sum_{Q_{r} \neq l / 2} v_{0}^{B}\left(Q_{r}\right) z^{\iota Q_{r}-0.5},
$$

where

$$
\underline{v}_{0}^{A}\left(Q_{r}\right)=2 \pi \iota \frac{\underline{P}^{A}\left(Q_{r}\right)}{\left(-\iota Q_{r}+0.5\right) D^{\prime}\left(Q_{r}\right)}
$$

and

$$
\underline{v}_{0}^{B}\left(Q_{r}\right)=2 \pi \iota \frac{\underline{P}^{B}\left(Q_{r}\right)}{\left(-\iota Q_{r}+0.5\right) D^{\prime}\left(Q_{r}\right)} .
$$

If we separate $Q_{r}$ into its real and imaginary parts as

$$
Q_{r 0}=g_{r 0}+\iota k_{r 0}
$$

then we see that the H--functions will have the following dependence on $z$ :

$$
H(z) \approx(z)^{-\left(k_{r 0}+0.5\right)} \exp \left[\lg _{r 0} \ln (z)\right]
$$

3D. Singularities in $H(z)$ at $z=0$

In this subsection we identify and briefly discuss the singularities in $\underline{H}(z)$. The nature and origin of these singularities are the same as in Part $I$, and the discussion given there is also generally applicable to the present case. The only difference between the plane-strain case as discussed in Part I and the present generalized plane-strain case arises because in 
the former case the integrand for $H(z)$ [see eq (54) of Part I] has $E(q)$ in the denominator, whereas in the present case it has the factor $(-\iota q+0.5)$.

The singularities in $\underline{H}(z)$ arise from (i) the homogeneous solution and (ii) the particular solution. These are discussed below:

(i) Singularities in the Homogeneous Solution

The form of the singularity is given by eq (104), where $\mathrm{k}_{\mathrm{ro}}$ satisfies the constraint given by eq (97). The exponent of q will have a value between 0 and -1 . This term will, in general, have a logarithmic oscillatory factor unless $g_{r o}$ is zero. This singularity will be present only if a $Q_{r}$ [a solution of eq (76)] exists which satisfies the constraint given by eq (97).

(ii) Singularities in the Particular solution

The singularities in the particular solution can be classified in two categories according to the nature of poles in the integrand for $\mathrm{H}(\mathrm{z})$ as:

(a) Singularities arising from simple poles. These are given by the first term in eq (81). The exponent of a singularity in this category can have a value between 0 and -0.5 . These terms will also have an oscillatory log factor unless Real $\left(Q_{r}\right)=0$.

(b) Singularities arising from higher order poles. These are given by the second term in eq (81). There is at least one second-order pole at $q=-\iota / 2$ arising from the factor $(-\iota q+0.5)$ and a root of $D(q)$, which gives rise to a logarithmic singularity as given by eq (86). This singularity has no oscillatory factor. 
If $D(q)$ has other degenerate roots, $\underline{H}(z)$ will have singularities of the form given by eq (87). The exponent of $z$ in this class of singularity may have a value between 0 and -0.5 . The logarithmic singularity may, in principle, have a power of up to 5. There will, in general, be a logarithmic oscillatory factor unless the real part of the root $Q_{r}$ is zero.

The exponents of all the singular terms are independent of the loading of the solid and depend only upon the elastic constants and the geometry of the solid. This is because the exponents of the singularities are characteristics of the matrix $M$, which does not depend upon the type of the loading. The loading only affects the RHS of eq (71). The weights of the singular terms in the homogeneous solution are also independent of the loading, as is apparent from eq (75). However, the weights of the singularities arising in the particular solution will depend upon the nature of the loading.

\section{Application to $\Sigma-5$ Grain Boundary in a Cubic Crystal}

In this section, as an example of the application of the formalism developed in this paper, we shall apply it to calculate the stress distribution in stainless steel containing a $\Sigma-5$ tilt grain boundary. We consider the same model which has been described in section 6 of Part I. We consider the solid subjected to an out-of-plane load such that $\varepsilon_{33}$ is equal to a constant $\eta$.

First we consider the solid to be infinite, having no free surfaces. The stress and the displacement field for this solid have been given in section 2. The expressions for various parameters such as $\underline{\gamma}$ and $\underline{\sigma}$ have been given in section 6 of part $I$.

As in Part I, we choose the units such that $\mathrm{C}_{44}=1$ for solid $A$ in the UHP and $\eta$ is taken to be unity. The calculated values of 
$u_{1}(\underline{x}), \tau_{11}(\underline{x}), \tau_{12}(\underline{x})$ and $\tau_{22}(\underline{x})$ can be easily obtained from eqs (34)-(39). We notice from these equations that the displacement field is a linear function of the distance from the origin, and the stresses are constants.

The calculated values of the components of the vectors $\underline{T}_{\infty 1}$ and $\underline{T}_{\infty 2}$ as defined by eqs $(36)-(39)$, and which are required for eqs (56) $-(59)$, are given below in units of $\eta$ [see eq (41)]:

$\underline{T}_{\infty 1}^{A}=(-1.3,0,0)$ and $\underline{T}_{\infty 1}^{B}=(-1.3,0,0)$

and

$\underline{\mathrm{T}}_{\infty 2}^{\mathrm{A}}=(0,-1.3,0)$ and $\underline{\underline{T}}_{\infty 2}^{\mathrm{B}}=(0,-1.3,0)$.

We note from the values given above that $\underline{T}_{\infty 2}$ is continuous across the interface, having the same values for solids $A$ and $B$. This is required by the boundary condition given by eq (6). In this particular case, because of high symmetry, $\underline{T}_{\infty 1}$ is also continuous and equal in magnitude to $\underline{T}_{\infty 2}$.

Now we consider the effect of a free surface in this model solid using the formalism presented in the preceding section. The particular solution for $\underline{H}(z)$ is given by eq (70) and the homogeneous solution by eq (74). In order to see whether the homogeneous solution is to be included or not, we have to solve eq (76) for its complex roots and see whether it has any roots which satisfy the constraint given by eq (97). The numerical method of calculation of the roots of eq (76) has been described in the next section. The two roots of eq (76) which have a positive imaginary part less than 0.5 are

and

$$
Q_{\text {ro }}=0.0272+0.4976 \mathrm{i}
$$




$$
\mathrm{Q}_{\mathrm{ro}}=0.0118+0.4994 \mathrm{i} \text {. }
$$

The corresponding singular terms in $\underline{H}(z)$ are given by eqs (99) and (100). The $z$-dependence of these singular terms is

$$
H(z) \approx z^{-0.9976} \cos (0.0272 \text { ln } z)
$$

and

$$
H(z) \approx z^{-0.9994} \cos (0.0118 \text { in } z)
$$

We notice from eqs (107)-(108) that these singular terms in the stress also have a logarithmic oscillatory factor but with a small magnitude.

The integrals for the particular solution in eqs (61) and (62) are calculated numerically. In the numerical calculations we have taken $y_{\infty}$ to be 10 and the limits of the q--integral from -10 to +10. For testing the convergence we follow the procedure given in Appendix II-A.

The calculated values of the displacement field $u_{1}(\underline{x})$ have been shown as function of $x_{1}$ and $x_{2}$ in a 3-D graph in figure II-2. We notice from this figure that, as required by the boundary condition given by eq $(44)$, the $u_{1}(\underline{x})$ is continuous across the interface. The behavior of other displacement components is similar to that of $u_{1}$.

The calculated values of the stress components $\tau_{12}(\underline{x})$ and $\tau_{22}(\underline{x})$ have been shown as functions of $x_{1}$ and $x_{2}$ in $3 D$ graphs in Figs II-3 and II-4 respectively. We notice from these figures that, as required by the boundary condition given by eq (45), these stress components are continuous across the interface. A similar behavior is shown by the stress component $\tau_{32}$. In addition, the stress component $\tau_{12}$ is zero at the free surface, 
which satisfies the boundary condition given by eqs (46) and (47). The stress component $\tau_{11}(\underline{x})$ is shown in figure II-5 as a function of $x_{1}$ and $x_{2}$. It is also seen to be zero at the free surface, which satisfies the boundary given by eqs (46) and (47). It is, of course, not continuous at the interface. A similar behavior is shown by the stress component $\tau_{31}$.

As is apparent from Figs II-3 through II-5, the stress components are singular at the origin. The nature of the singularities has been discussed in the preceding section. The exponents of the two singular terms which arise from the homogeneous solution are determined from the values of the roots in accordance with the discussion given in section 3D. The behavior of these singular terms is given by eqs (107) and (108).

In order to identify the singularities in the particular integral, we have to calculate the roots of eq (76) in the lower half of the complex $q$-plane. As remarked in the previous section, eq (76) has one root at $q=-\iota / 2$. The corresponding singularity in the H-function will therefore be a log singularity as given by eq (86). The other root of eq (76) with negative imaginary parts and which satisfy the constraint given by eq $(84)$ is

$$
Q_{p r}=0.0163-0.3342 i
$$

The corresponding singular terms in $\underline{H}(z)$ will be given by eq (81). The nature of this singularity is

$$
H(z) \approx z^{-0.1658} \cos (0.0163 \text { in } z) \text {. }
$$

We find that this singularity also has a logarithmic oscillatory factor with a small coefficient. As remarked in section 3D, the exponents of these singularities are independent of the loading 
and are the same as obtained for the plane-strain problem in section 6 of Part I.

\section{Application to Fiber-Reinforced Laminated Composites}

In this section we further apply the formalism developed in this paper to calculate the displacement field and the stress distribution in a fiber-reinforced laminated composite containing a free surface and subjected to an out-of-plane load. We shall consider a planar interface separating two different fiber-reinforced layers, terminating at a stress-free edge, which is taken to be normal to the interface. As mentioned in the introduction, this problem is important for studying the failure of composite materials.

A real fiber-reinforced laminated composite contains several layers and interfaces separating them. In general, the interfaces are not planar. However, we make the usual simplifying assumptions about the material by considering it as a bimaterial composite with a single planar interface. We assume that both the layers are homogeneous and anisotropic, and have no boundaries except for the interface separating them and the stress-free edge. In spite of various approximations, the analytical model calculations are very useful because they provide a good understanding of the underlying physical principles of failure of composite materials. Moreover, they provide a basis for an efficient numerical approach for calculation of the stress distribution in a real material [14].

Thus we are considering a model in which the two solids, A and $B$, in figure II-1 are the same material except for the orientation of the fibers. As in the earlier papers, we specify the fiber orientations in terms of $\theta$, the angle of rotation about the $Y$-axis $\left(x_{2}\right.$-axis) in figure II- 1 . The angle $\theta$ is measured from the $\mathrm{Z}$-axis as shown in figure II-6. The elastic constants of the 
two solids, $A$ and $B$, are therefore related by a rotation transformation given by the orthonormal matrix,

$$
S=\left(\begin{array}{ccc}
\cos \theta & 0 & \sin \theta \\
0 & 1 & 0 \\
-\sin \theta & 0 & \cos \theta
\end{array}\right)
$$

The bimaterial composite in figure II-1 is usually specified in terms of the fiber orientations as $\left(\theta, \theta^{\prime}\right)$, which means that the fibers in solids $A$ and $B$ are at angles $\theta$ and $\theta^{\prime}$, respectively, from the $Z$ axis as shown in figure II-6. The orientation $\theta=0$ refers to the case when the fibers are parallel to the $z$ axis.

In this paper we shall consider composites with the orientations: $(0,90),(30,-30)$ and $(45,-45)$ where the angles are given in degrees. There is a strong interest in the engineering properties of composites with these orientations. We shall consider a typical high-modulus graphite/epoxy material. The set of engineering elastic moduli for this material for $\theta=0$, as given in $[10]$, is

$$
\begin{aligned}
& E_{1}=E_{2}=1.447 ; E_{3}=13.79 ; G_{12}=G_{23}=G_{31}=0.586 ; \\
& v_{21}=v_{31}=v_{32}=0.21
\end{aligned}
$$

where $E_{i}$ and $G_{i j}$ are the Young's and shear moduli respectively in units of $10^{7} \mathrm{kPa}$, and $v_{i j}$ are the Poisson's ratio. The above constants are based upon the assumption of hexagonal symmetry (transverse isotropy) and are the same as used in [10]. The calculated values of the corresponding elastic constants, which we shall refer to as set $I$, are given below (in units of $\mathrm{MN} / \mathrm{m}^{2}$ ):

$\underline{\text { Set }} \underline{I}$ 


$$
\begin{aligned}
& c_{11}=c_{22}=15.25 ; c_{12}=0.329 ; c_{13}=c_{23}=0.389 ; \\
& c_{33}=139.5 ; c_{44}=c_{55}=c_{66}=0.586 .
\end{aligned}
$$

In addition to the assumption of transverse isotropy, the elastic constants of set I are based upon the following assumption, referred to as the Pipes-Pagano approximation:

$$
G_{12}=G_{31} \text { and } v_{21}=v_{32} \text {. }
$$

The validity of the assumption in eq (112) has been questioned by Kriz [13], who has obtained a set of elastic constants without making this assumption. This set, which will be referred to as set $I I$, is given below in units of $\mathrm{MN} / \mathrm{m}^{2}$ along with the corresponding set of engineering elastic moduli in units of $10^{7} \mathrm{kPa}$ :

\section{$\underline{\text { Set }} \underline{\text { II }}$}

$$
\begin{aligned}
& C_{11}=C_{22}=1.347 ; C_{12}=0.674 ; C_{13}=C_{23}=0.618 ; \\
& C_{33}=14.17 ; C_{44}=C_{55}=0.579 ; C_{66}=0.336 ; \\
& E_{1}=E_{2}=1.002 ; E_{3}=13.79 ; G_{12}=0.336 ; G_{23}=G_{31}=0.579 ; \\
& v_{21}=0.491 ; v_{31}=v_{32}=0.306 .
\end{aligned}
$$

In our calculations, we have included both the sets of elastic constants given above. The calculations are carried out exactly along the lines described in section 3 and section 4 . The main parameters of the results are given in table 2. This table gives the values of $\mathrm{p}_{\alpha^{\prime}}$ the roots of the stroh determinant, as defined by eqs (A.19) and (A.20) of Part $I$, for $\alpha=1,2$ and 3 and for solids $A$ and $B$. These roots are purely imaginary and agree with those calculated in [11]. 
TABLE 2

Results for composite materials with different fiber orientations

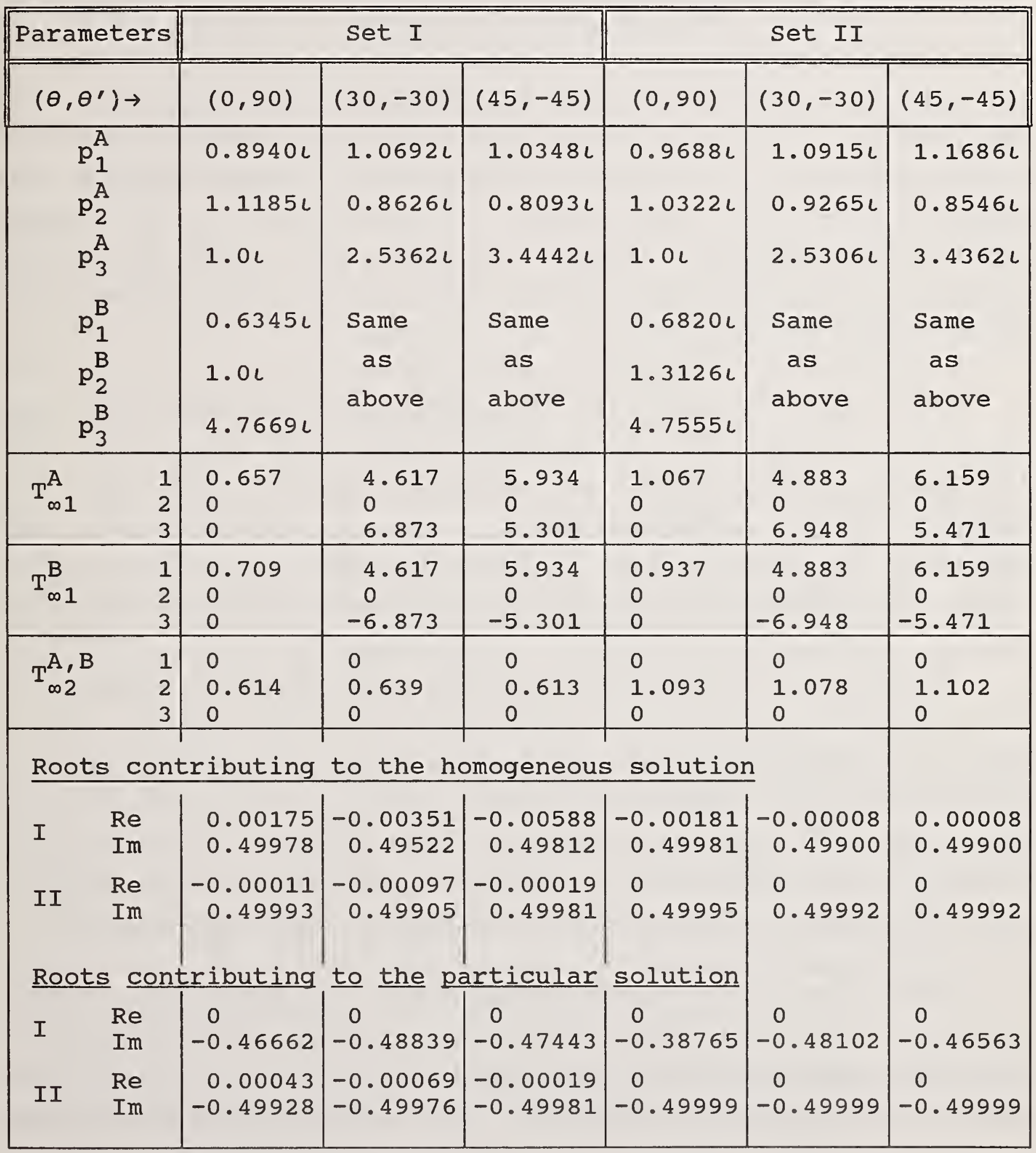


Further, Table 2 gives $\underline{T}_{\infty 1}$ and the corresponding values of $\underline{T}_{\infty 2}$ as defined in eqs (36)-(40). The value of $\underline{T}_{\infty 1}$ is needed for calculating the RHS of eqs (78) and (92). We immediately notice that both the stress components are constants as shown in section 2. In addition, $\underline{T}_{\infty 2}$ has the same value in UHP and LHP as required by the continuity condition given by eq (6).

Table 2 also gives the permissible values of $Q$ in the exponent iQ-0.5 of $z$ in the definition of $H(z)$. These values of $Q$ are the roots of eq (76). The values of $Q$ which contribute to the homogeneous solution given by eqs (99) and (100) are those which satisfy the constraint given by eq (97). The values of $Q$ which arise in the particular solution are those which satisfy the constraint given by eq (84).

The roots arising in the particular solution are not explicitly needed for the calculation of the stress and the displacement field since, as explained in the text, they are included in the integral over $q$ in eq (77). However, they represent the singularities in $\mathrm{H}(\mathrm{z})$ and the stress field at the origin as represented by eq (81) and discussed in section 3D (ii).

Table 2 shows that there are two roots which contribute to the singularity in $\mathrm{H}(\mathrm{z})$ as described in section 3D(ii). Out of these, the exponent of the singularity arising from the first root agrees with that obtained by Zwiers, et al., [10] and Ting and Chou [11] for the same composite. This root has no real part; therefore, as described in section $3 \mathrm{D}(\mathrm{i} i)$, it will have no logarithmic oscillatory factor in agreement with [10] and [11].

The second root is very close to $-\iota / 2$. As described in the section 3, it can be shown analytically that eq (76) has a root at $-\iota / 2$. The numerical method used in the present calculations seems to give one more root at about the same value which shows that the 
root at $-\iota / 2$ is doubly degenerate. This degeneracy has not been reported by earlier authors. Since this root of eq (76) is itself doubly degenerate, the integrand on the RHS of eq (77) will have a third order pole. As discussed in section $3 \mathrm{D}(\mathrm{ii})$, therefore, this implies that $H(z)$ has a $[\ln (z)]^{2}$ singularity at $z=0$ and not just $\ln (z)$. The weight of the singularity is given by eq (87).

The roots which contribute to the homogeneous solution are explicitly introduced in the total solution for $H(z)$, as given by eqs (99) and (100). From Table 2 we see that in addition to the singularity in the particular solution, $H(z)$ will have two more singular terms of the type $z^{-1+\varepsilon}$ where $\varepsilon$ is very small--of the order of $10^{-3}$ for one root and $10^{-5}$ for the other root. The two roots are apparently degenerate, which indicates a higher order pole at $\iota / 2$. However, as described in section 3 , the root at $\iota / 2$ does not contribute to the stress.

The complex roots of eq (76), as quoted in Table 2 and in section 4 for the cubic case, were obtained by the Muller's method by using a library program on the mainframe computer at NIST. Equation (76) has roots at $\pm \iota / 2$ which are known analytically. These were given as known roots in the Muller's method and were not calculated numerically.

The numerical method, however, gave two apparently new roots close to this value as shown in Table 2 . Since these roots just satisfy the constraint given by eq (97), they were included in the calculation. We could not ascertain with more than an accuracy of $10^{-6}$ that these are independent roots (possibly degenerate with the one at $(/ 2)$ or the same root at $\iota / 2$ which is being picked up by the numerical method with a round-off error.

In order to verify the independence of these roots, we first divided the LHS of eq $(76)$ by $\left(z^{2}+0.25\right)$, which should remove the 
known roots at $\iota / 2$ and $-\iota / 2$. Then we applied the Muller's method again to obtain the remaining roots. The method still gave the roots as given in the table, which suggested, within an error of $10^{-5}$, that these are indeed independent and degenerate roots. However, the uncertainty is not serious because, as shown in section 3, the contribution of the root at $1 / 2$ to the stress is zero. The contribution of the roots in Table 2 , which are close to this value, is also quite insignificant.

We calculated the integral over $q$ in eq (77) numerically between the limits -8 to 8 . The convergence of this integral has been discussed in Appendix II-A. The value of $Y_{I}$ was taken to be 10. The calculated values of $u_{1}, \tau_{12}, \tau_{22}$ and $\tau_{11}$ for $(0,90)$ composite, using set I elastic constants, have been shown as functions of $x_{1}$ and $x_{2}$ as $3 D$ graphs in figure II-7 through figure II-10 respectively. The same quantities for $(30,-30)$ and $(45,-45)$ composites using set I elastic constants and for $(0,90),(30,-30)$ and $(45,-45)$ composites using set II elastic constants have been shown in figure II-11 through II-30.

We have given the above results for composites with different orientations because of a strong engineering interest in these materials. These curves should be useful in detailed numerical studies of the stress distribution in real composites.

We see from the various figures that, as required by the boundary conditions, the stress components $\tau_{12}$ and $\tau_{22}$ and the displacement field are continuous across the interface, whereas, in general, $\tau_{11}$ is not. However, in case of $(30 /-30)$ and $(45 /-45)$ composites, $\tau_{11}$ is also continuous across the interface. This is a consequence of the mirror symmetry across the interface. Apparently for the same reason, $u_{1}$ has an extremum (maximum or minimum) at the interface along the $Y$ - axis in these materials. 
Although the displacement field is continuous across the interface, in case of the $(0 / 90)$ composite $u_{1}$ has a very large gradient at the interface along the $\mathrm{Y}$-axis. This is a consequence of the strong elastic anisotropy of the solids in UHP and LHP in the composite. This can be understood qualitatively as follows.

The stiffness is very large in a direction parallel to the fibers and very low in a direction perpendicular to the fibers. In case of the $(0 / 90)$ composite, the fibers in the UHP and the LHP are parallel to the $\mathrm{Z}$ - and the $\mathrm{x}$-axes respectively. In the UHP $\mathrm{u}_{1}$ is the displacement component in a direction perpendicular to the fibers, which is against relatively weak elastic forces. On the other hand, in the LHP, $u_{1}$ is in a direction parallel to the fibers, which is against relatively strong elastic forces. The two are therefore very different. The same argument explains the large difference between the values of $\tau_{11}$ in the UHP and LHP in the $(0 / 90)$ composite.

The stress components $\tau_{11}$ and $\tau_{12}$ are zero at the free surface, as required by the boundary conditions given by eqs (46) and (47). All the stresses are singular at the origin, as shown by the peaks in the figures. These peaks appear to be finite in the figures, because in actual calculations we calculate the stress close to the origin but not exactly at the origin for obvious numerical reasons.

\section{Summary of Results and Discussion}

In this part we have generalized the elastic plane-strain Green's function method given in Paper I and used the method of Part I to calculate the stress distribution and the displacement field in a bimaterial composite subjected to a generalized plane-strain loading. The stress components are expressed in terms 
of a function $H(z)$, which is given by the solution of a generalized inhomogeneous Hilbert equation. The main effort is in the determination of the function $H(z)$. The final result contains two parts: a particular solution which is expressed in the form of a closed integral representation, and a homogeneous solution which consists of a finite number of terms.

The integral for the particular solution can be evaluated analytically by using contour integration. This gives the result in the form of a series, with each term representing the contribution of the poles in the integrand. The series contain singular as well as nonsingular terms and may not be a convergent series. The advantage of the series representation is that it helps in precisely identifying the singularities in the stress--their weights (the coefficient of a singular term) as well as exponents. The earlier work in this field, in general, only attempted to identify the singular terms and calculate their exponents. As suggested in [10] and [11], the weight of the singularity has to be obtained by a numerical solution of the boundary value problem.

In these calculations we have considered only one free surface in the solid and assumed the solid to extend to infinity in other directions. The effect of outer boundaries of the solid, which in real cases would have irregular shapes, is very difficult to include in an analytical calculation. This can be easily done by using a numerical technique such as the finite element method.

However, a purely numerical technique becomes inefficient and unreliable in a region where the solution has singularities and discontinuities. In such cases, the proper procedure would be to combine the analytical and the numerical techniques by using enriched finite element method [14] or taking the exact analytical solution near the origin and joining it by numerical solution in 
the outer regions. The formulation given in the present paper should be particularly suitable for this purpose.

In this paper we have shown that the stress will have singularities of the form $z^{-\delta}(0<\delta<0.5)$ and $\ln (z)$ (as well as its higher powers) arising from the particular solution and $z^{-1+\delta}$ arising from the homogeneous solution. The homogeneous solution will contribute only in certain cases as given in section $3 \mathrm{D}$ (i). In addition, depending upon the material constants, the singularity may have a logarithmic oscillatory factor which is similar to that associated with interfacial cracks (see, for example, Paper II).

We did not find the logarithmic oscillatory factor in case of the laminated composites which is in agreement with the earlier work on these materials. However, in case of the cubic solids described in the preceding section, we did find this factor, although with a small magnitude. Our result on the nature of the singularities of the type $z^{-\delta}$ and $\ln (z)$ in laminated composites agrees with that obtained in some earlier papers $[10,11]$. However, the singularities with exponents between -0.5 and -1.0 have not been identified in the earlier papers.

The existence of the homogeneous solution in our result is due to a boundary condition which we impose on the solution of the Hilbert equation. This condition requires the displacements to be single-valued. It is obviously a physical requirement which comes naturally in our method of solution because otherwise the Hilbert equation will not have a unique solution. This seems to have been missed in the earlier published work. This may be the reason why the singularities arising in the homogeneous solution with exponents between -0.5 and -1.0 have not been identified so far.

It can be easily seen analytically that if the exponent of the 
singularity in the homogeneous solution is exactly -1 , it will not contribute to the stress. In the case of the laminated composites which was considered in section 5, the only terms which we found in the homogeneous solution have exponents very close to -1 (imaginary part of $Q$ close to 0.5 ). These terms do not make $a$ significant contribution to the stress and therefore do not invalidate the results published in the earlier papers on such laminated composites. Even in the cubic case which was discussed in section 4 , the exponents of these terms are quite close to -1 .

Regarding the singularities in the particular solution, we seem to obtain a degenerate root at $Q=-\iota / 2$ as shown in Table 2 . As remarked in the preceding section, this implies a singularity of the type $\left[\begin{array}{ll}l n & z\end{array}\right]^{2}$. The possibility of such singularities has been acknowledged in [10] but not actually included in the earlier calculations. In our method, we do not need to isolate the singularities in the particular solution which are all automatically included in the integral representation.

One of the advantages of our method is that we do not have to calculate the exact roots of eq (76) except for the homogeneous solution when it exists. In these cases, we have only to search for the roots in a finite range as given by eq (97). Moreover, even when we have to calculate the roots, we need to solve only a $6 \times 6$ determinantal equation. In the earlier methods, $[10,11]$ one has to solve a $12 \times 12$ determinantal equation. The main disadvantage of this method is that it cannot account for the other surfaces and interfaces which exist in a real solid. In real cases, a combination of a numerical and the present technique, as suggested earlier, should provide an efficient method for analyzing the stress distribution. 


\section{Appendix II-A}

\section{Transform of a Constant and Some Numerical Considerations}

In this appendix, we describe the transform of a constant which is required for calculating the RHS of eq (63). In this context we shall also discuss some aspects of the numerical convergence which are relevant to the calculations in this paper.

Let $c$ denote a constant, which is expressed in terms of its transform $c(q)$ as

$$
c=\int_{-\infty}^{\infty} c(q) y^{\ell q-0.5} d q
$$

Following the method given in Appendix I-B of Part $I$, we obtain the following inverse relation for $c(q)$

$$
c(q)=\frac{1}{2 \pi} \int_{0}^{\infty} c y^{-\iota q-0.5}
$$

which is an equation of the same form as eq (64) or eq (65).

We evaluate the integral in eq (A.1) in the limits 0 and $Y_{\infty}$ and take the limit as $y_{\infty}$ goes to infinity. The integral is then elementary, and we obtain the following expression for $c(q)$

$$
c(q)=-\frac{c}{2 \pi \iota} \frac{1}{(q+0.5 \iota)}\left(y_{\infty}\right)^{-\iota q+0.5}
$$

In order to verify that this gives the correct transform of the constant $C$, we substitute for $c(q)$ from eq (A.3) in eq (A.1). obviously the inverse relation is valid only if the following 
integral is independent of $y$ in the limit $y_{\infty} \rightarrow \infty$ and is equal to unity; that is,

$$
-\frac{1}{2 \pi \iota} \int_{-\infty}^{\infty} \frac{1}{(q+0.5 \iota)}\left(y_{\infty} / y\right)^{-\iota q+0.5} d q=1
$$

The integral on the LHS of eq (A.4) can be easily evaluated by taking a semicircular contour in the lower half plane as shown in figure I-4 of Part I. In the limit of large $y_{\infty}$ the contribution of the semicircle will vanish, and the integral can be seen to be unity as required by eq (A.4) for all values of $y$.

Equation (A.4) can be used for testing the convergence of the numerical integration over $q$ in eqs (61) and (62). In order to carry out the numerical integration for $\mathrm{H}(\mathrm{z})$, we have to choose finite but large values of $\mathrm{y}_{\infty}$ and the limits of the integration for $q$. We also evaluate numerically the integral in eq (A.4) with the same values and check whether the integral is acceptably close to unity.

One problem which arises in this context is that if the limits of integration for $q$ are taken to be too large, the integrand in eq (61) may become numerically unstable. Some matrix elements of the matrix M(q) contain factors like exp( $\pm \pi q)$, which will become too large near the upper or the lower limit of $q$ in the integration. The matrix will then become ill-conditioned and its calculated inverse may contain large numerical errors. On the other hand if the limits of $q$ are taken to be too small, the integral may not converge in the sense required by eq (A.4).

The stress $\underline{T}_{1}(\underline{x})$ as calculated from eqs (58) and (59) will be zero at $x_{1}=0$ only if eq (A.4) is satisfied. It is therefore necessary to choose optimum values for the limits of $q$ so that eq 
(A.4) is satisfied without making the matrix M(q) ill-conditioned. To make the matters worse, the convergence also depends upon values of $y$ in eq (A.4). This would suggest that different limits for the q-integral have to be chosen for different values of $y$. This is not advisable because it might introduce artificial discontinuities in the result.

One way out of this difficulty, which we have used in our calculations, is the following. We choose reasonable values for $y_{\infty}$ and the limits of $q$ and evaluate the integral over $q$ for $H(z)$ in eqs (61) and (62) using these constants. Then we calculate the integral in eq (A.4) by using the same values of $y, y_{\infty}$, limits of $q$ and the integration interval. The calculated value of the integral in eq (A.4) is taken to be the effective value of unity. We then divide $\underline{H}(z)$ by this effective value of unity. The errors due to lack of convergence are then largely cancelled out from the result. This is indicated by the fact that $\underline{T}_{1}(\underline{x})$ as calculated from eqs (58) and (59) will become zero at $x_{1}=0$ in accordance with the free-surface boundary condition. 



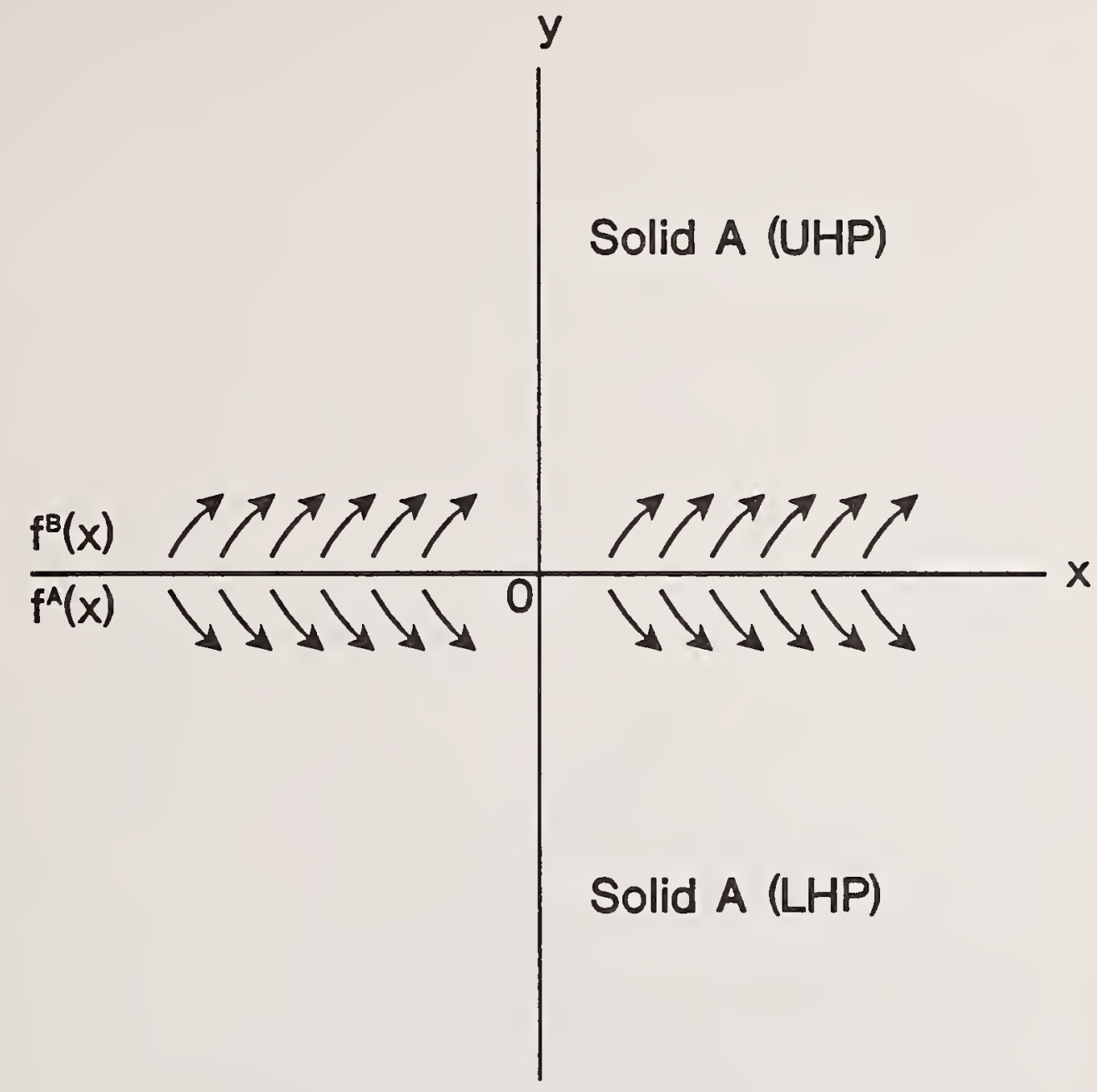

Figure II-1: An infinite bimaterial composite containing a plane interface and the coordinate system used in these calculations. The force functions $f^{A}(x)$ and $f^{B}(x)$ are applied just outside the UHP and LHP respectively. 


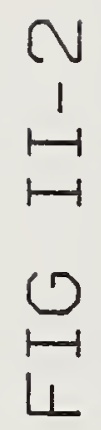

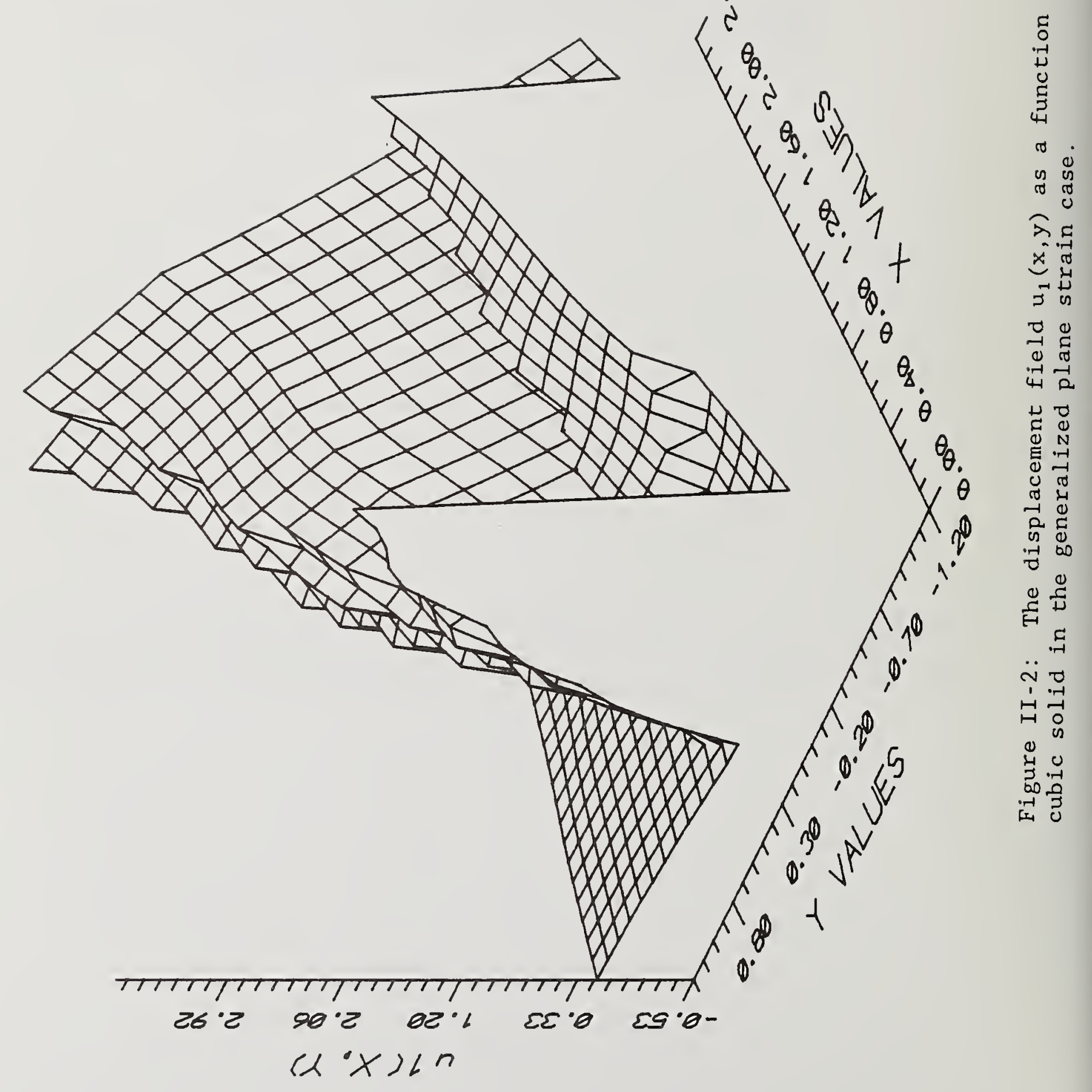




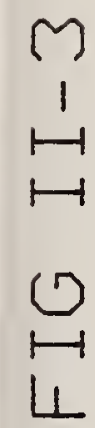

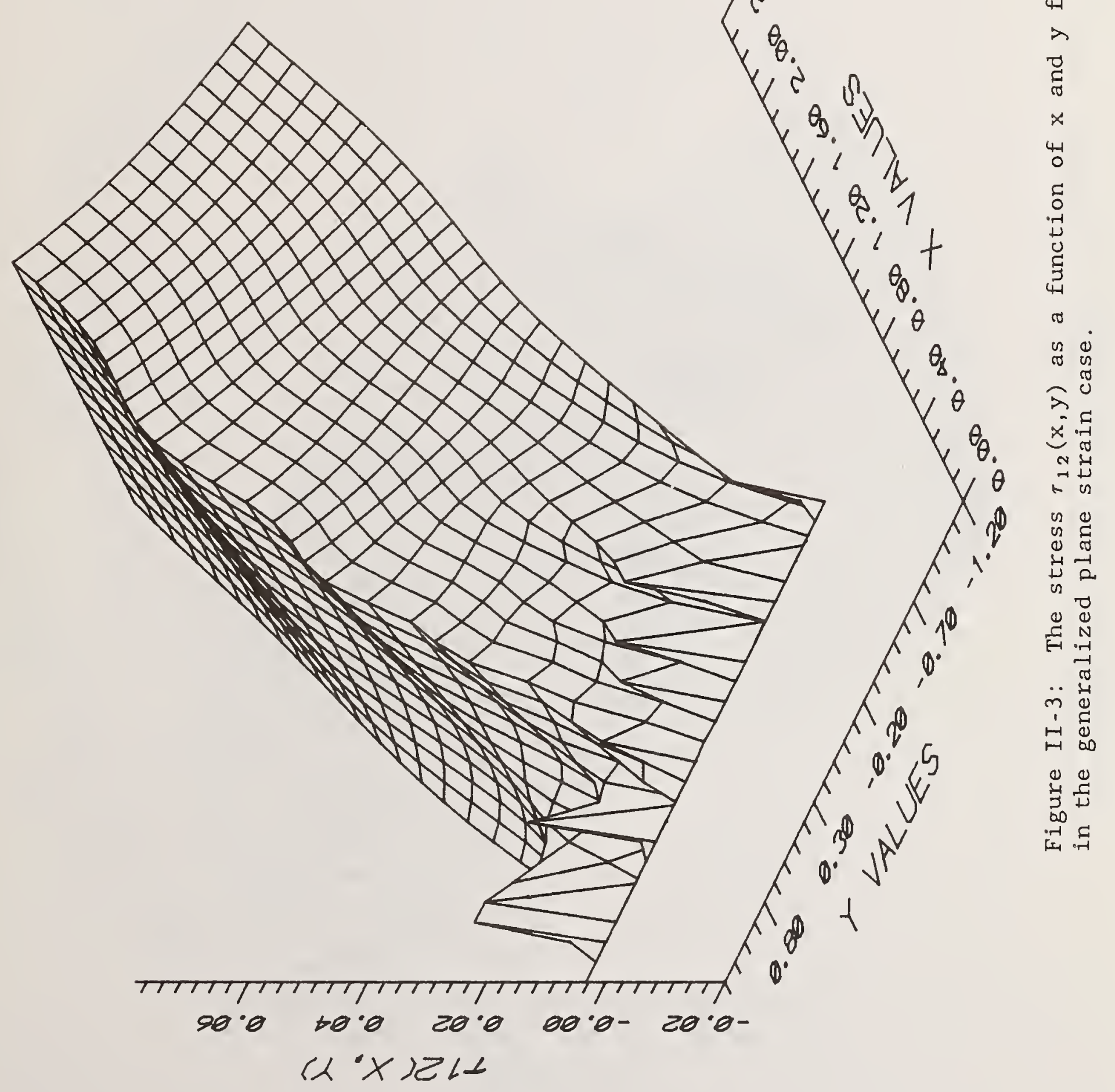




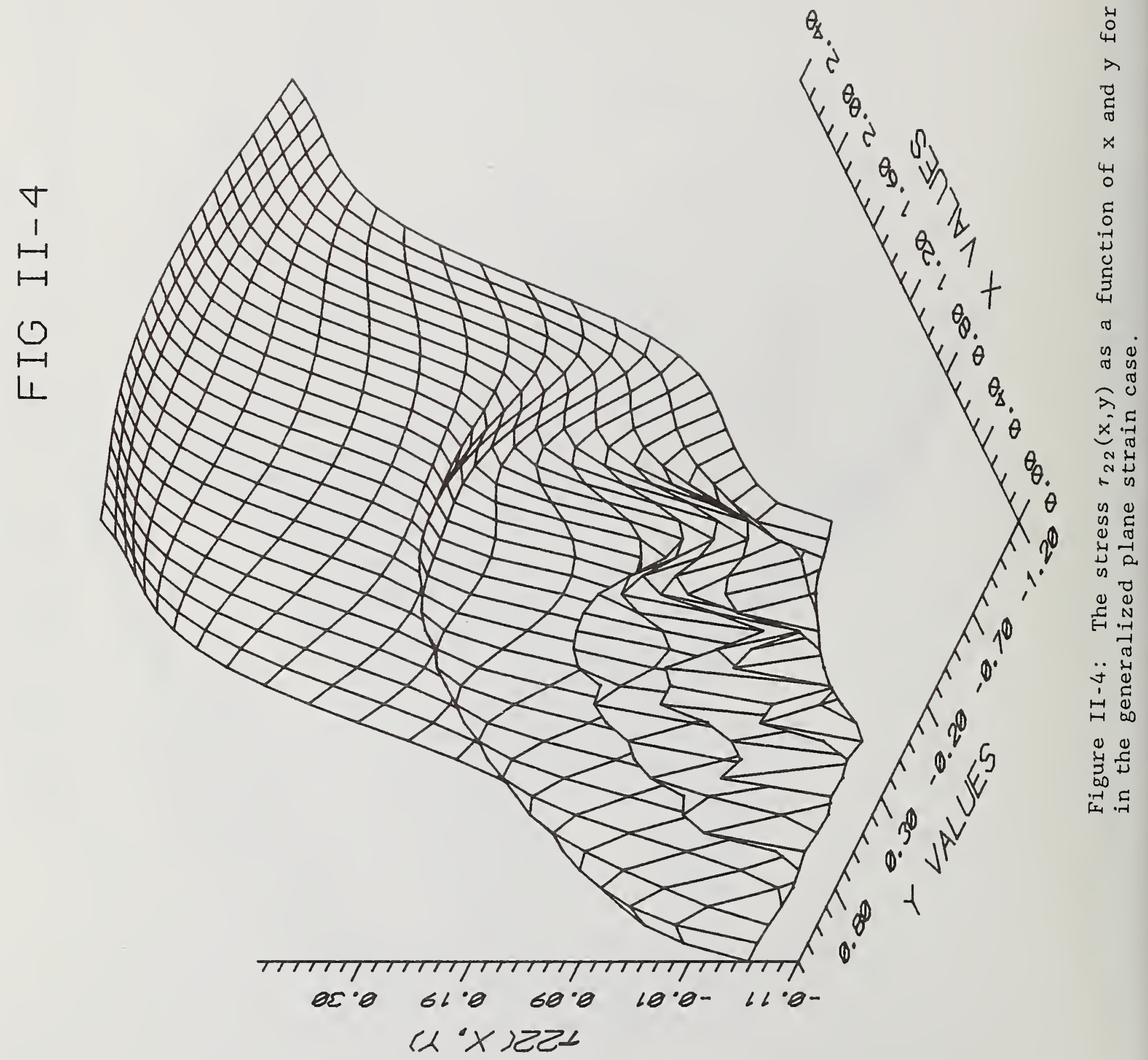


$\sqcup$
1
$\mapsto$
$\mapsto$
0
$\stackrel{H}{L}$

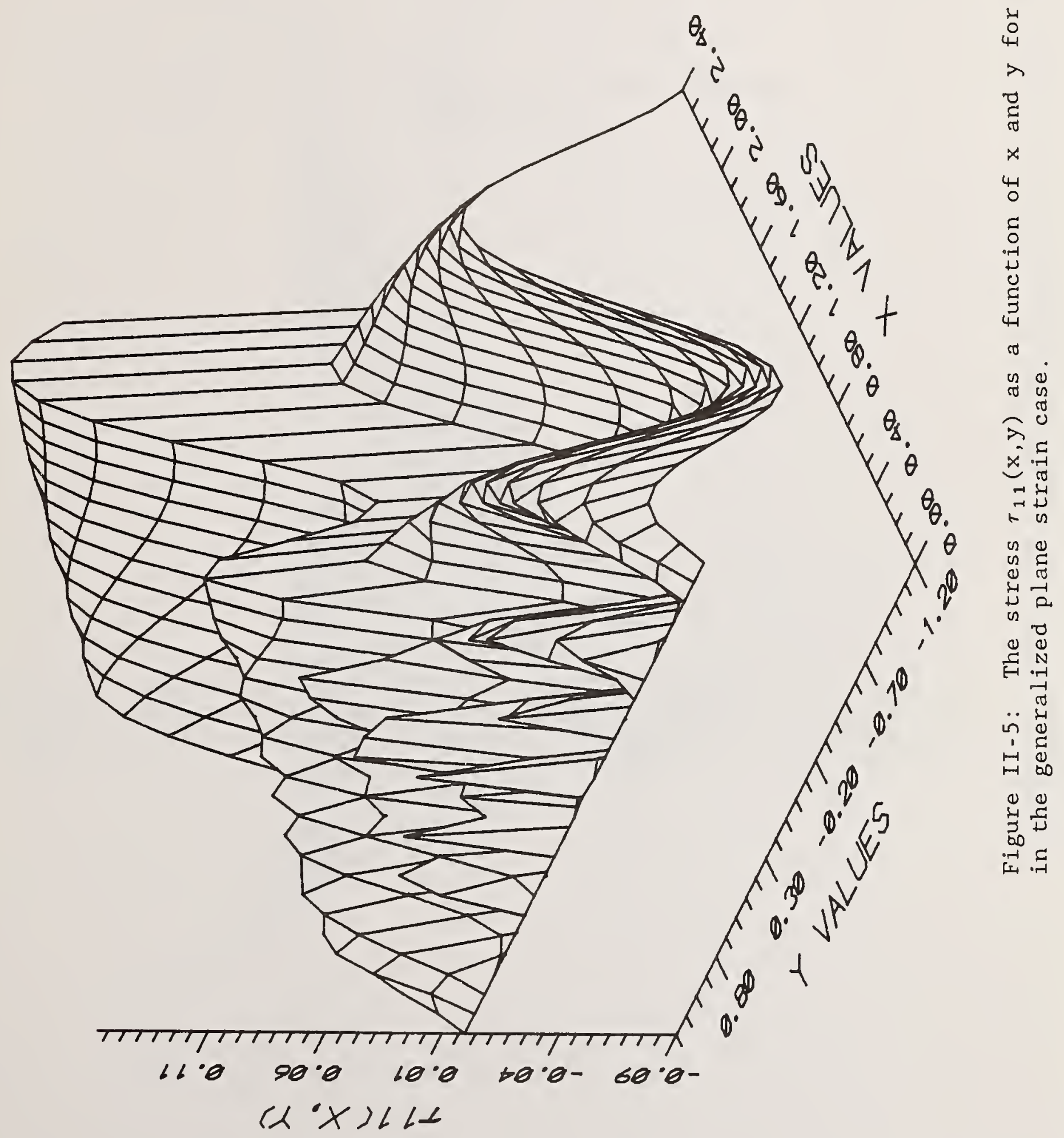




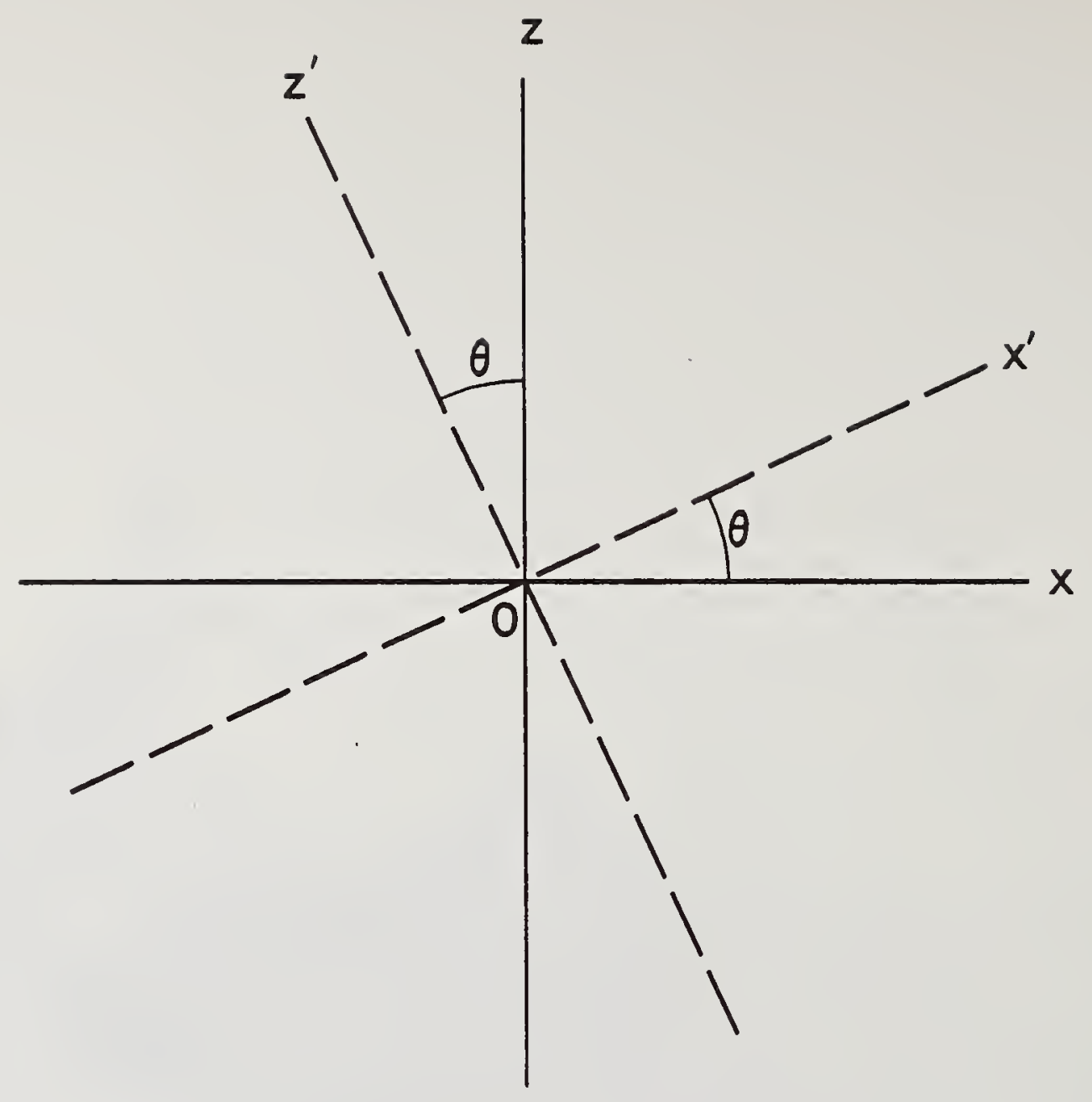

Figure II-6: The rotation angle of the fibers with respect to the $Z$ or the $X$ axis. The rotation is about the $Y$ axis which is normal to the plane of the paper. At 0 orientation, the fibers are parallel to the $\mathrm{Z}$ axis. At orientation $\theta$, the fibers are parallel to $\mathrm{OZ}^{\prime}$. 


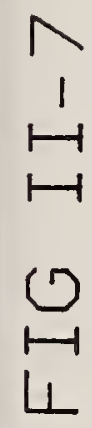

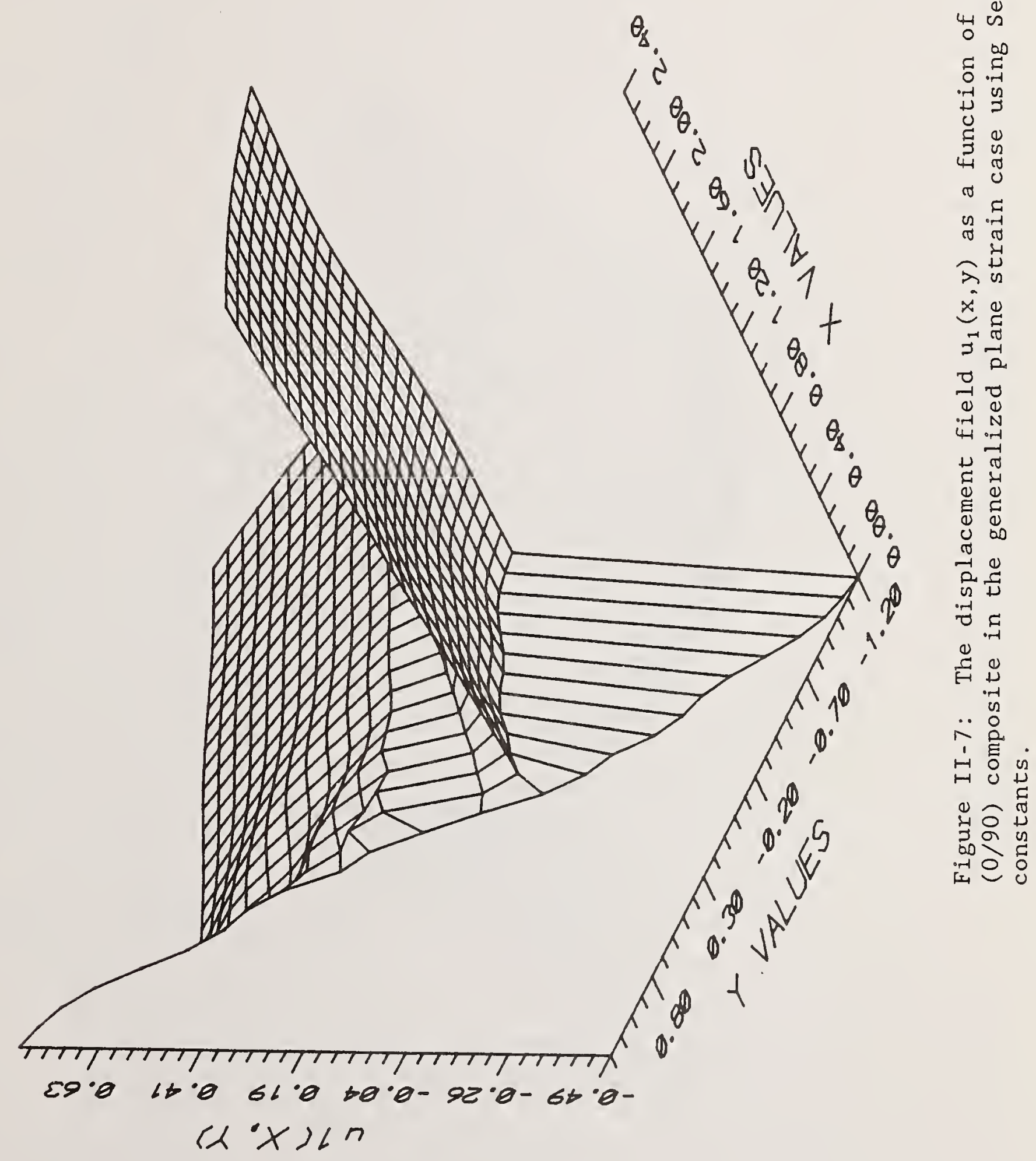




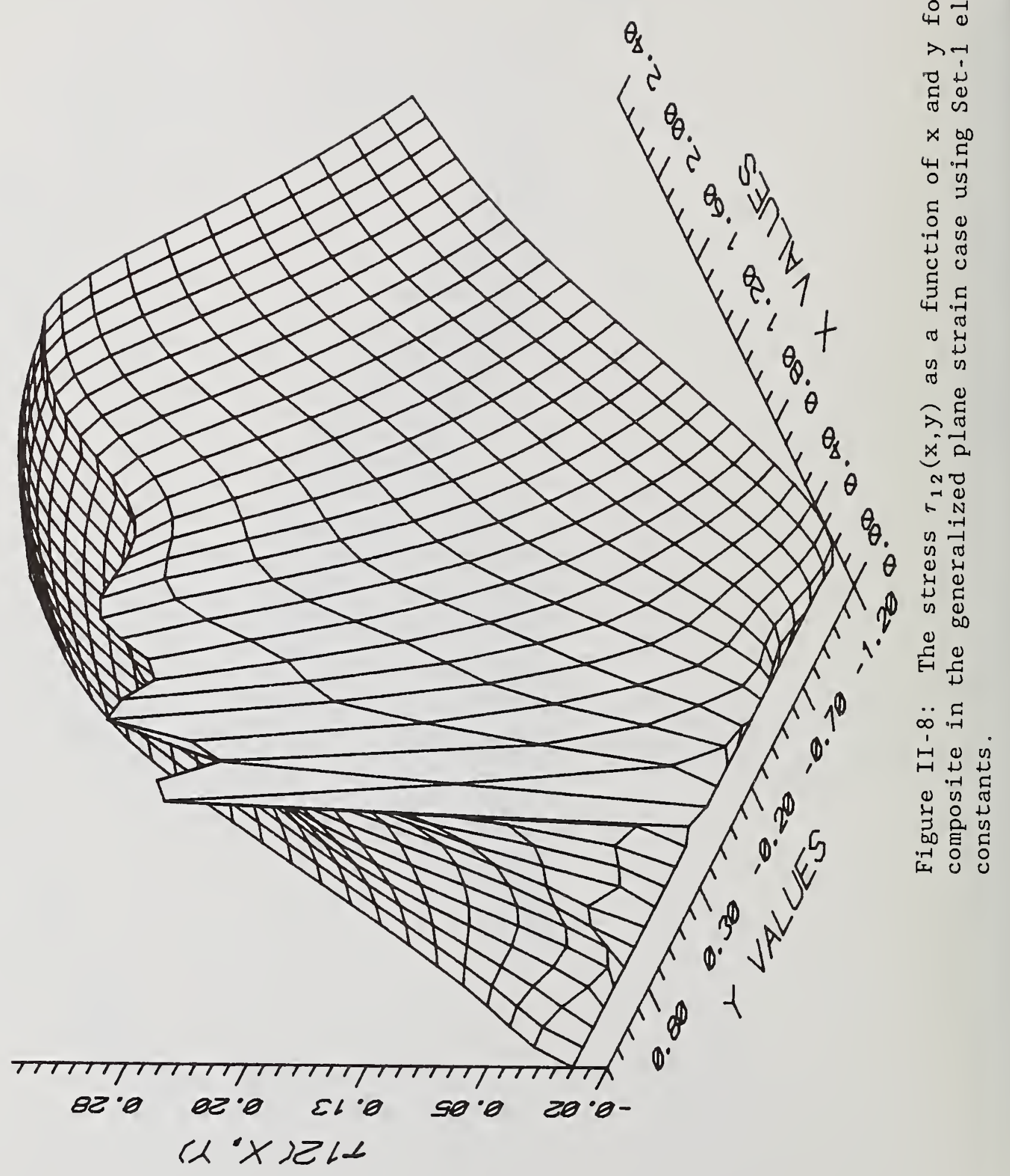


$a$
1
$\mapsto$
$\mapsto$
0
$\stackrel{\leftrightarrow}{-}$
$\stackrel{L}{L}$

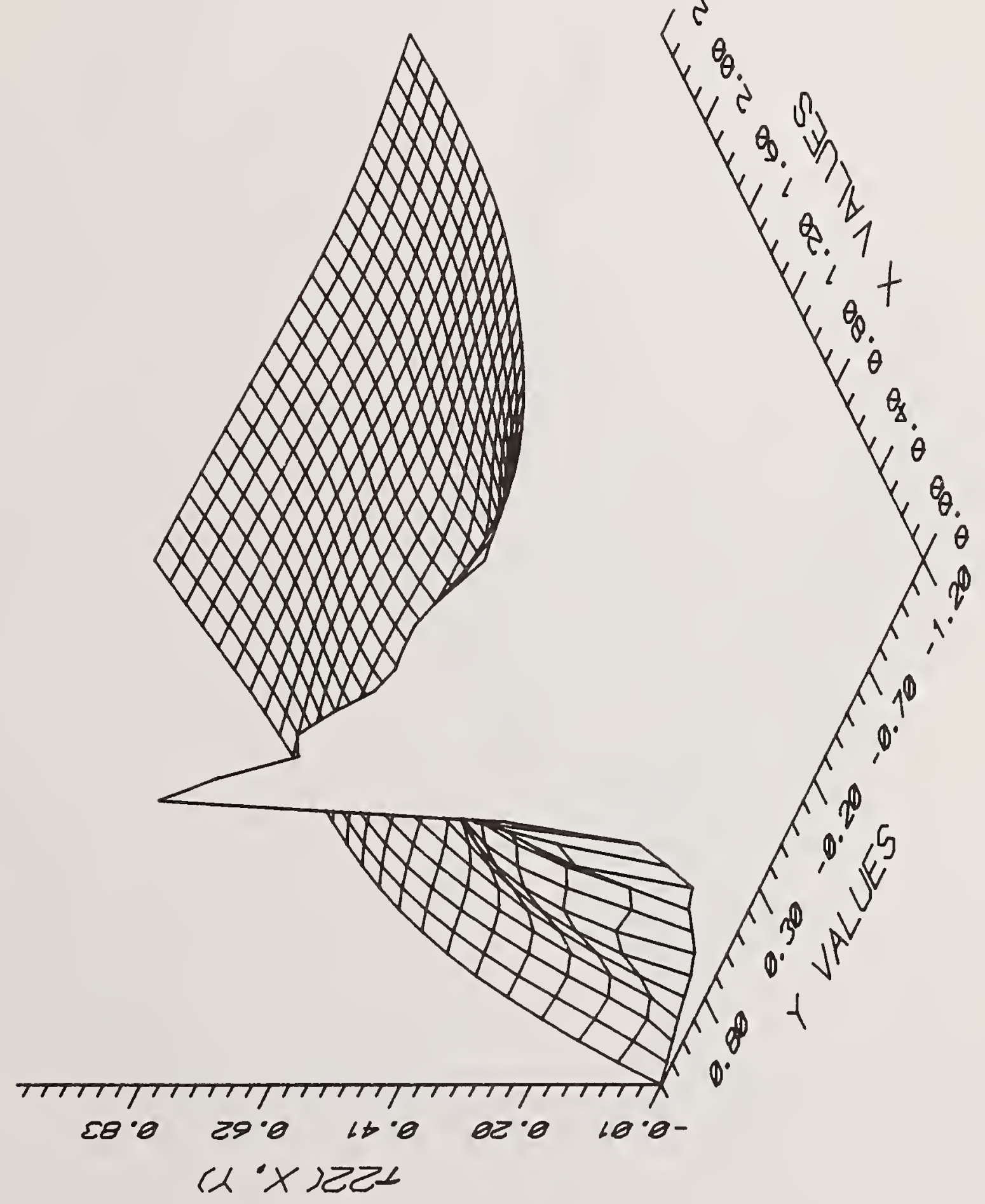

ฮ̊

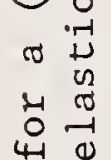

$\lambda-$

च्

$\times$ b

出

5

If

引

फ न

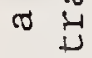

थ 0

久

$x-1$

NO

ND

$+N$

प 2 न

d

(n)

突 $\frac{⿱ 0}{D}$

$\ddot{\sigma} . F$

붕

$\stackrel{\leftrightarrow}{*}$

$\begin{array}{cc}0 & 0 \\ 0 & 0 \\ 3 & 0 \\ 0 & 0 \\ 0-1 & 0 \\ \text { c. } & 0\end{array}$ 


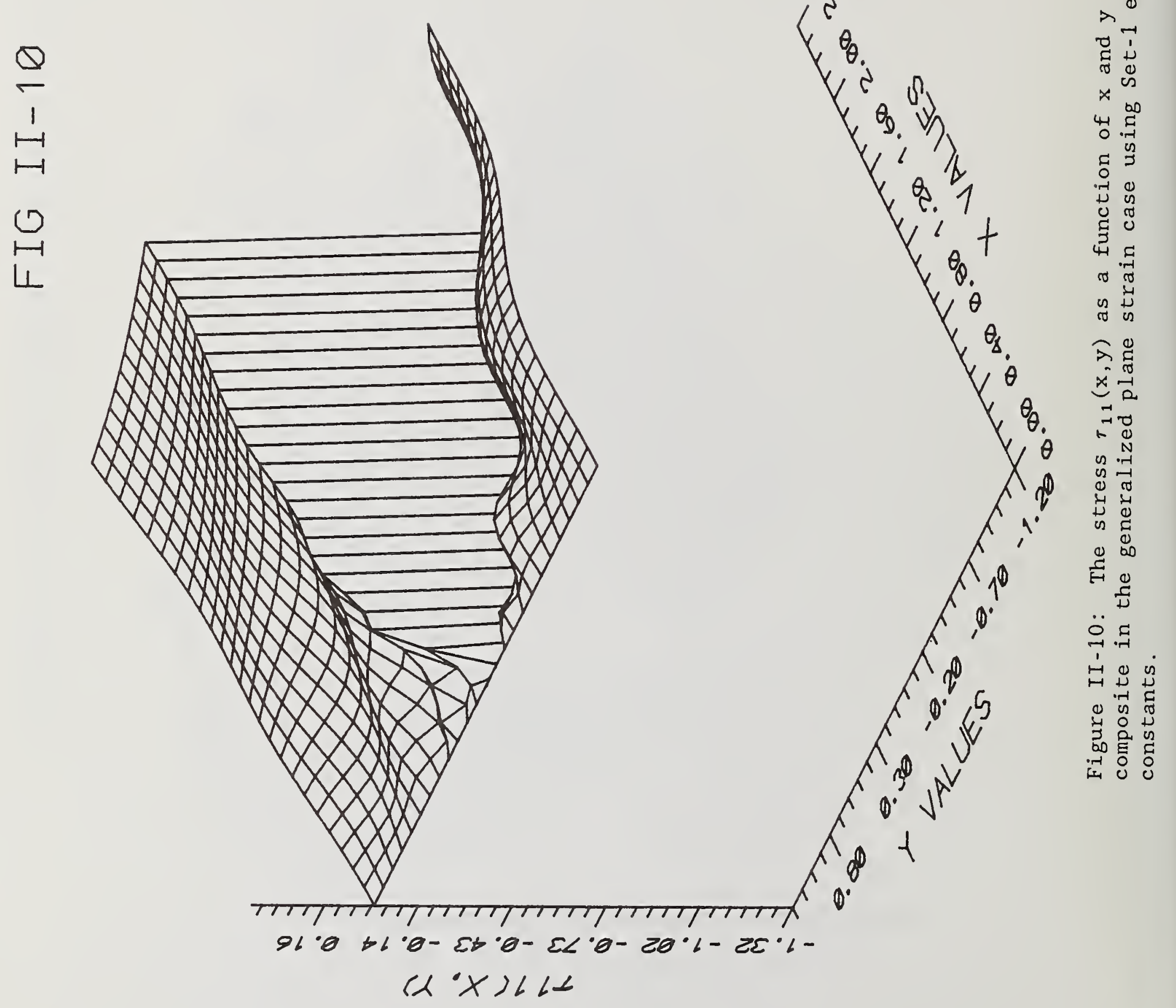




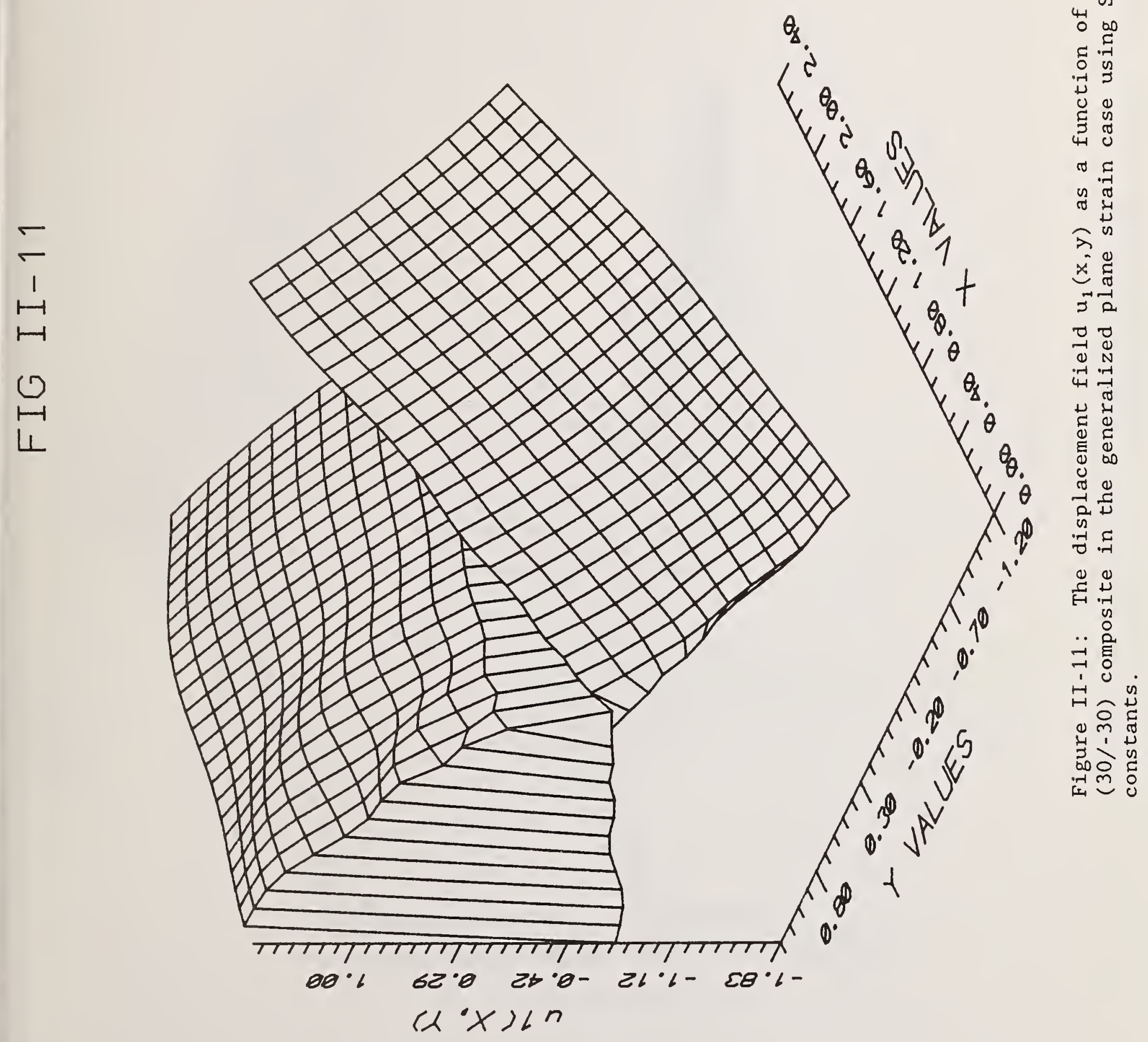




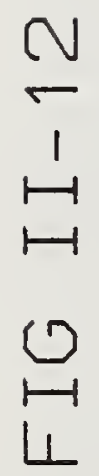

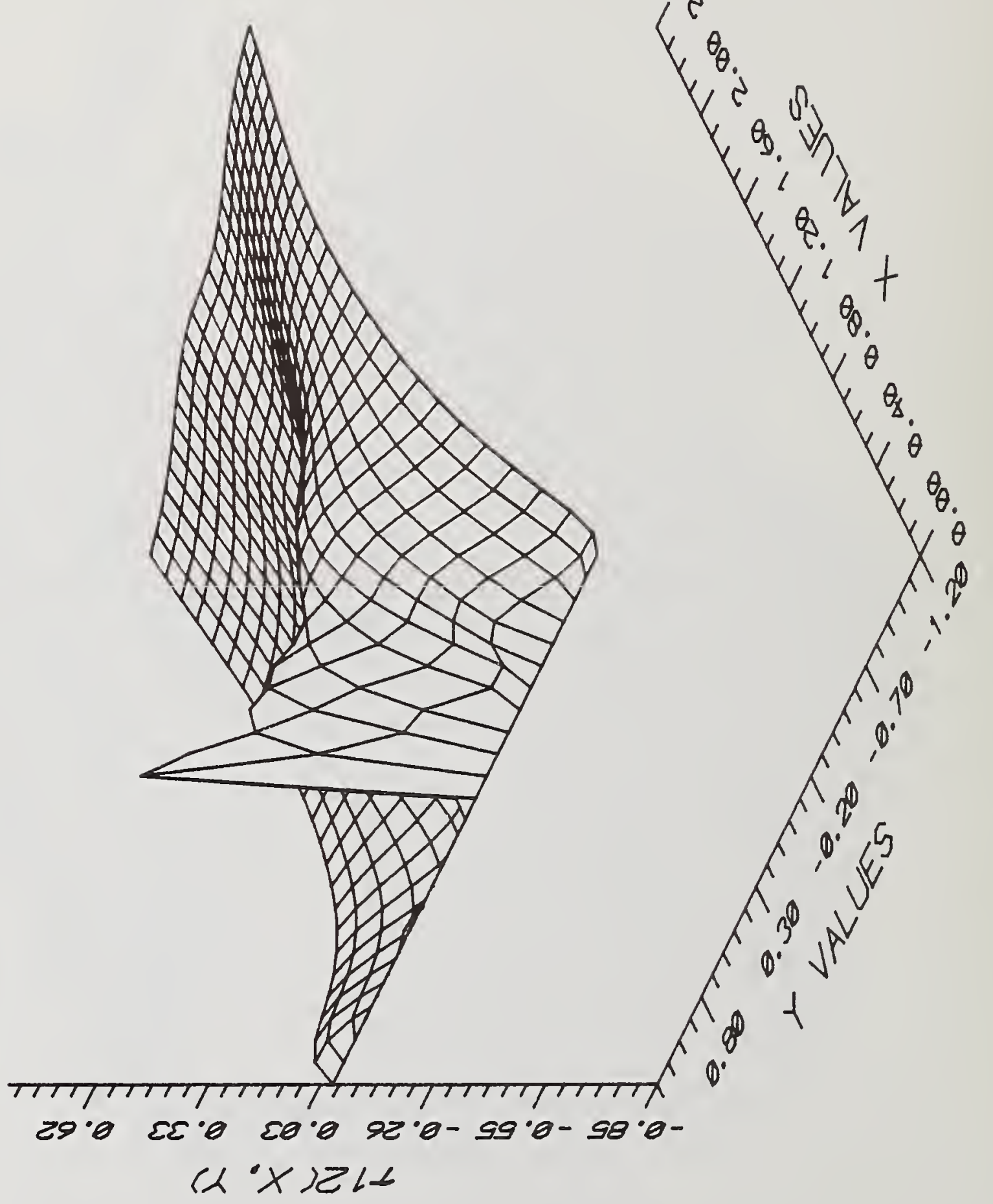

$\begin{array}{cc}4 & 2 \\ 0 & 0 \\ 4 & 0\end{array}$

$\lambda$

¿

$x$

4.

ㄷํㅇ

望

䓀

$\pi \frac{\pi}{\pi}$

थ

久离

$x$

ND

0
0
0

(2) $\frac{\pi}{\pi}$

㝳

(2)

(c)

E

$\ddot{\sim}$

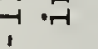

تص山

(1) थ

崖 을

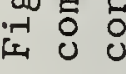




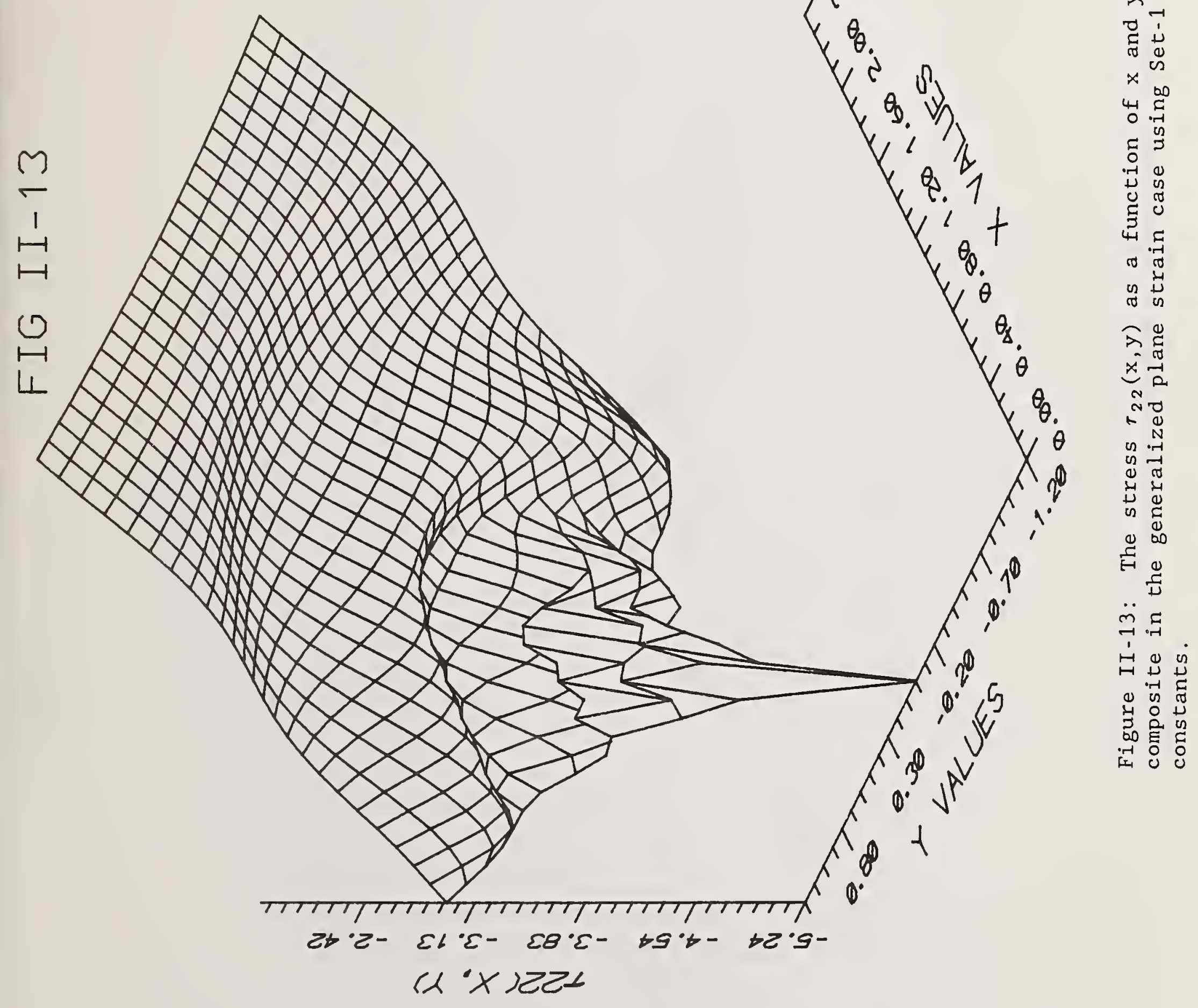




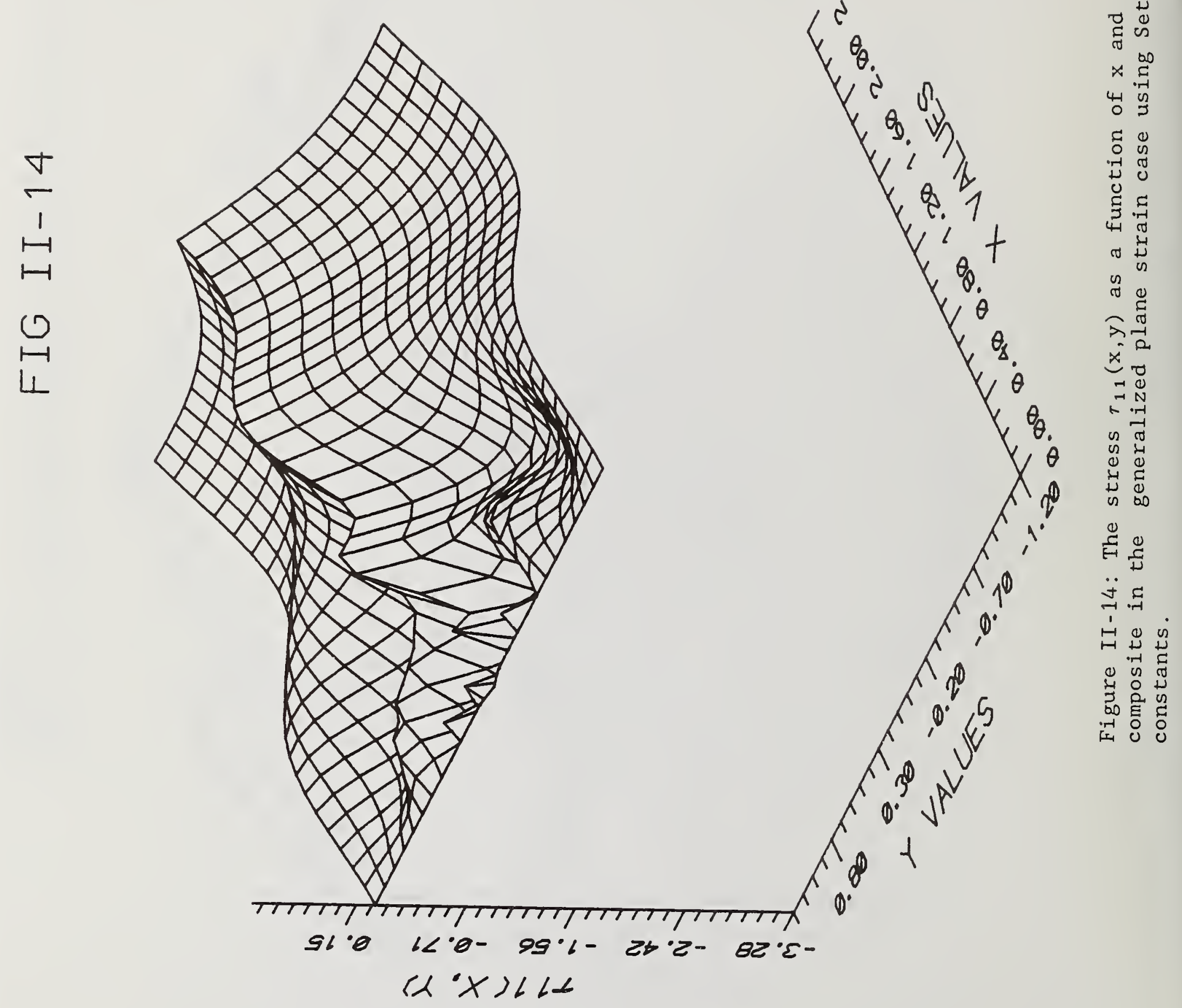


5
1
$H$
$H$
0
$\stackrel{H}{L}$

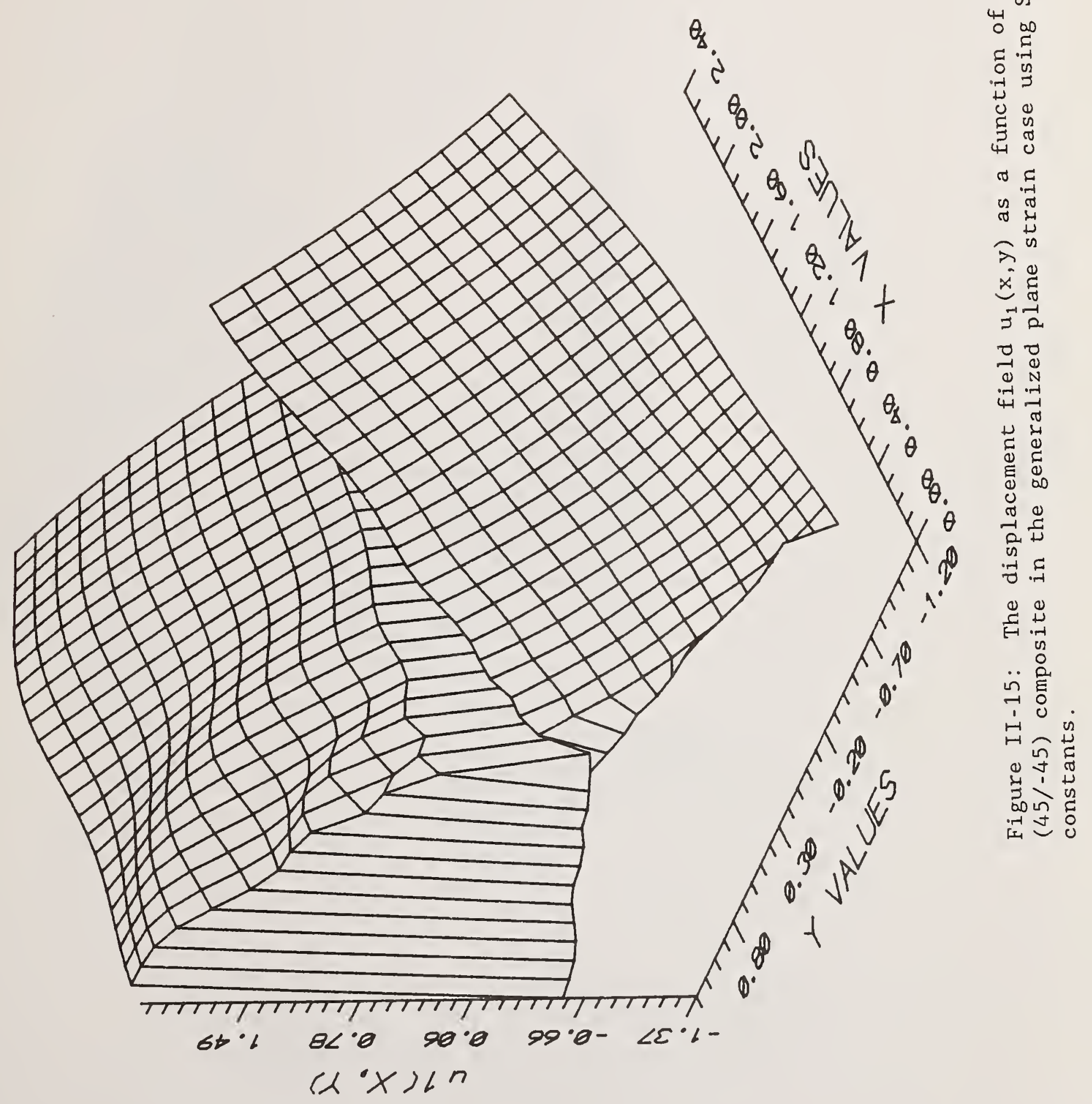




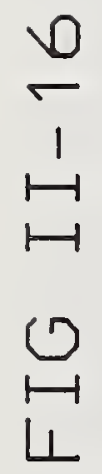

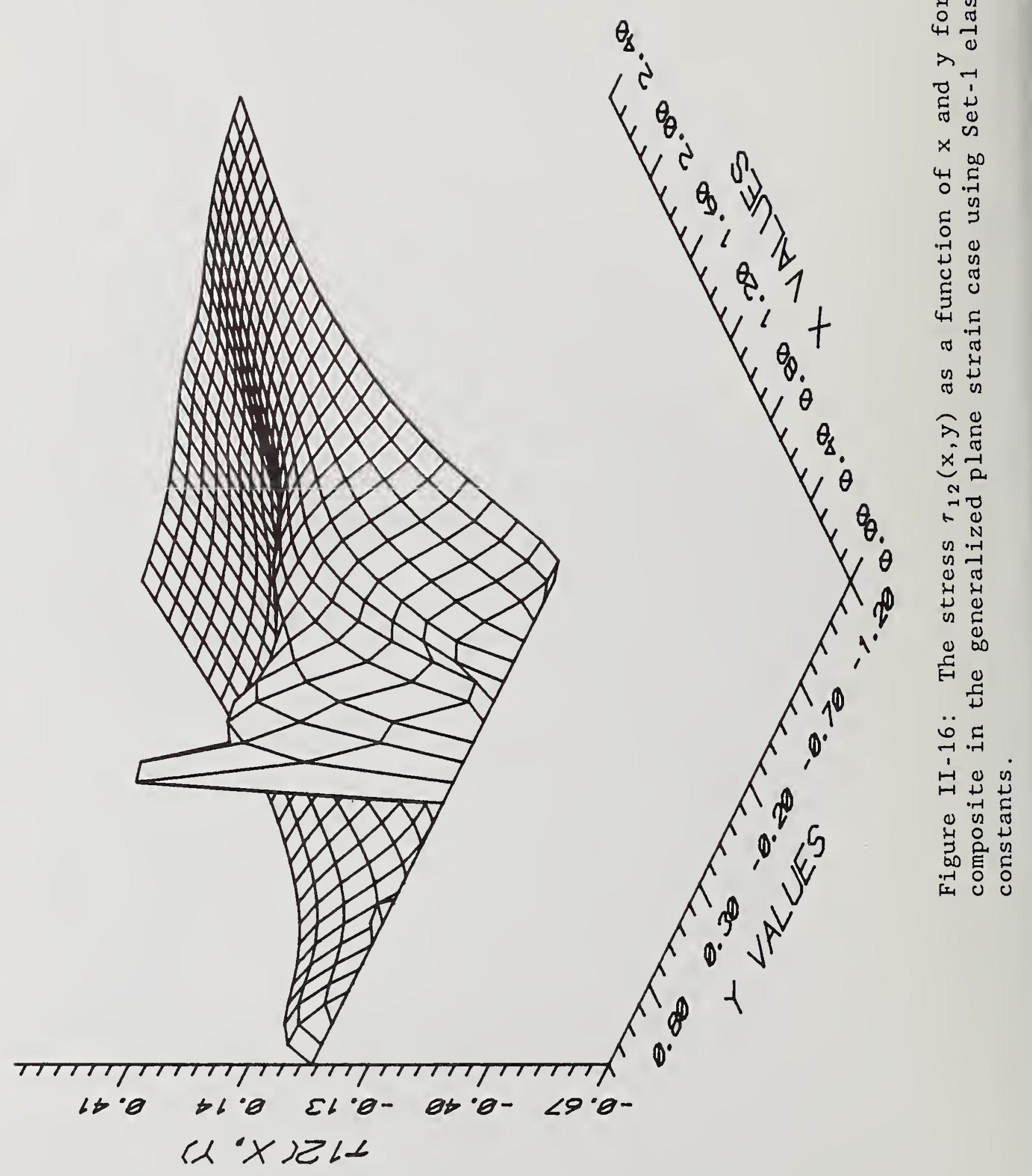




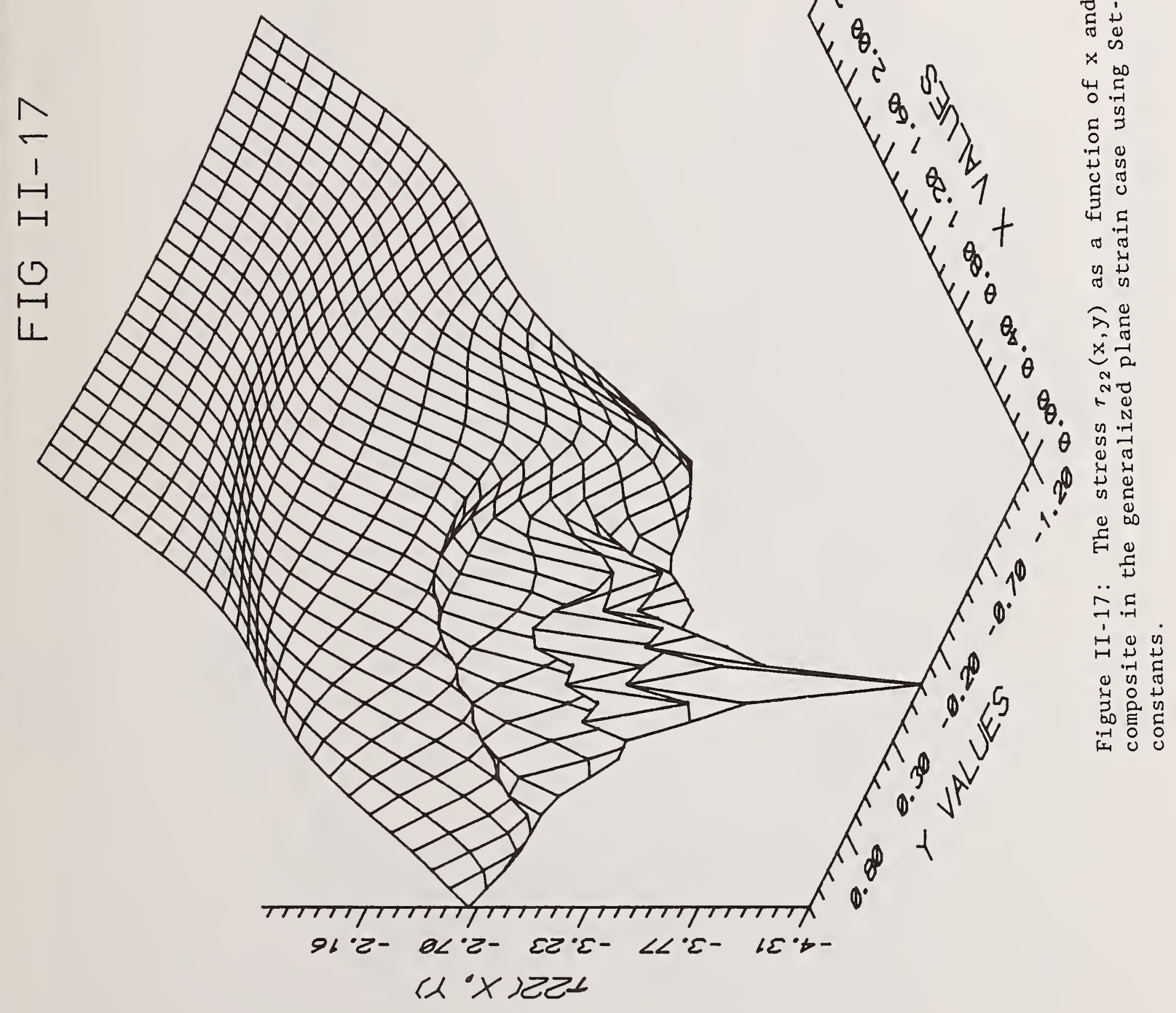




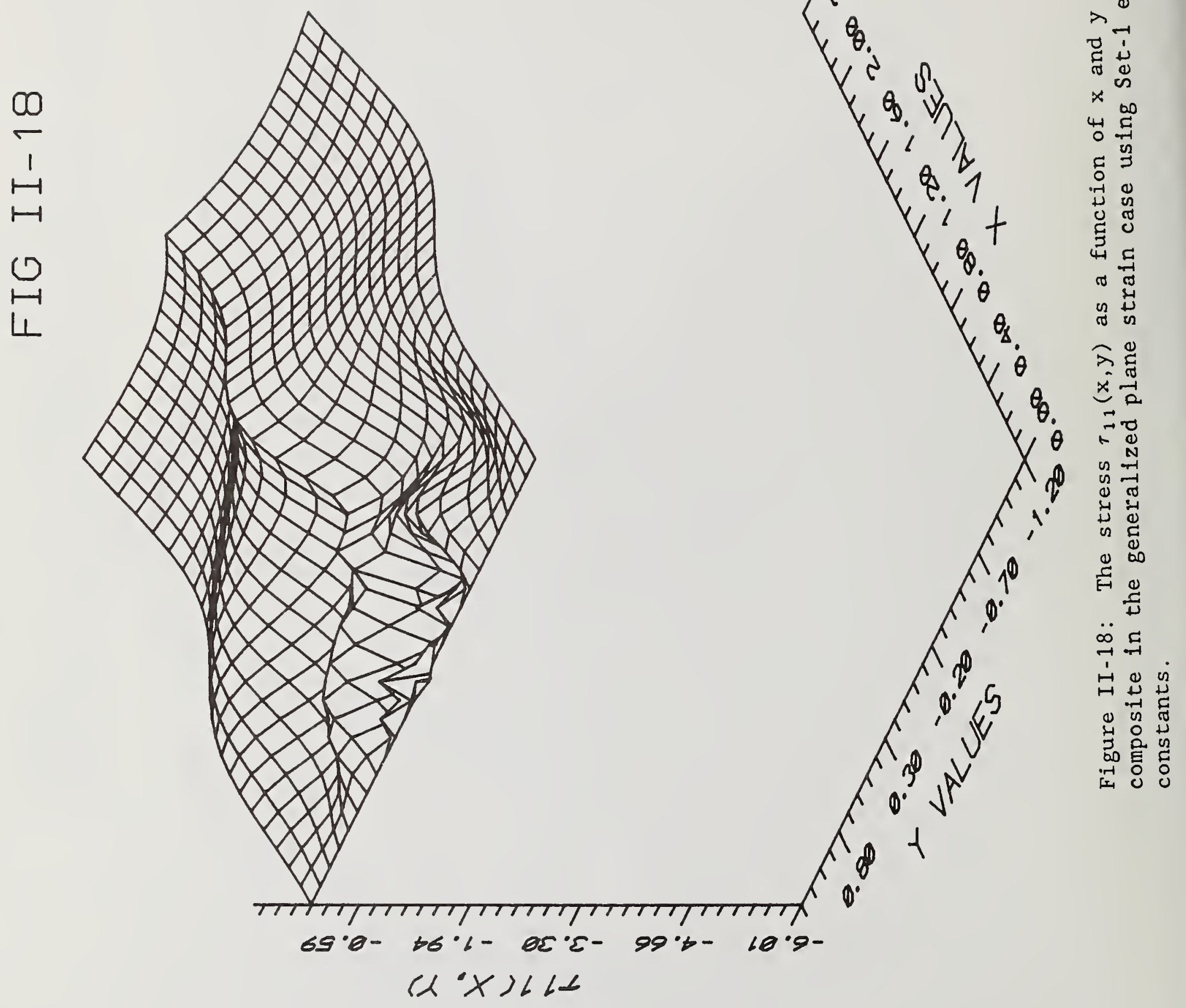




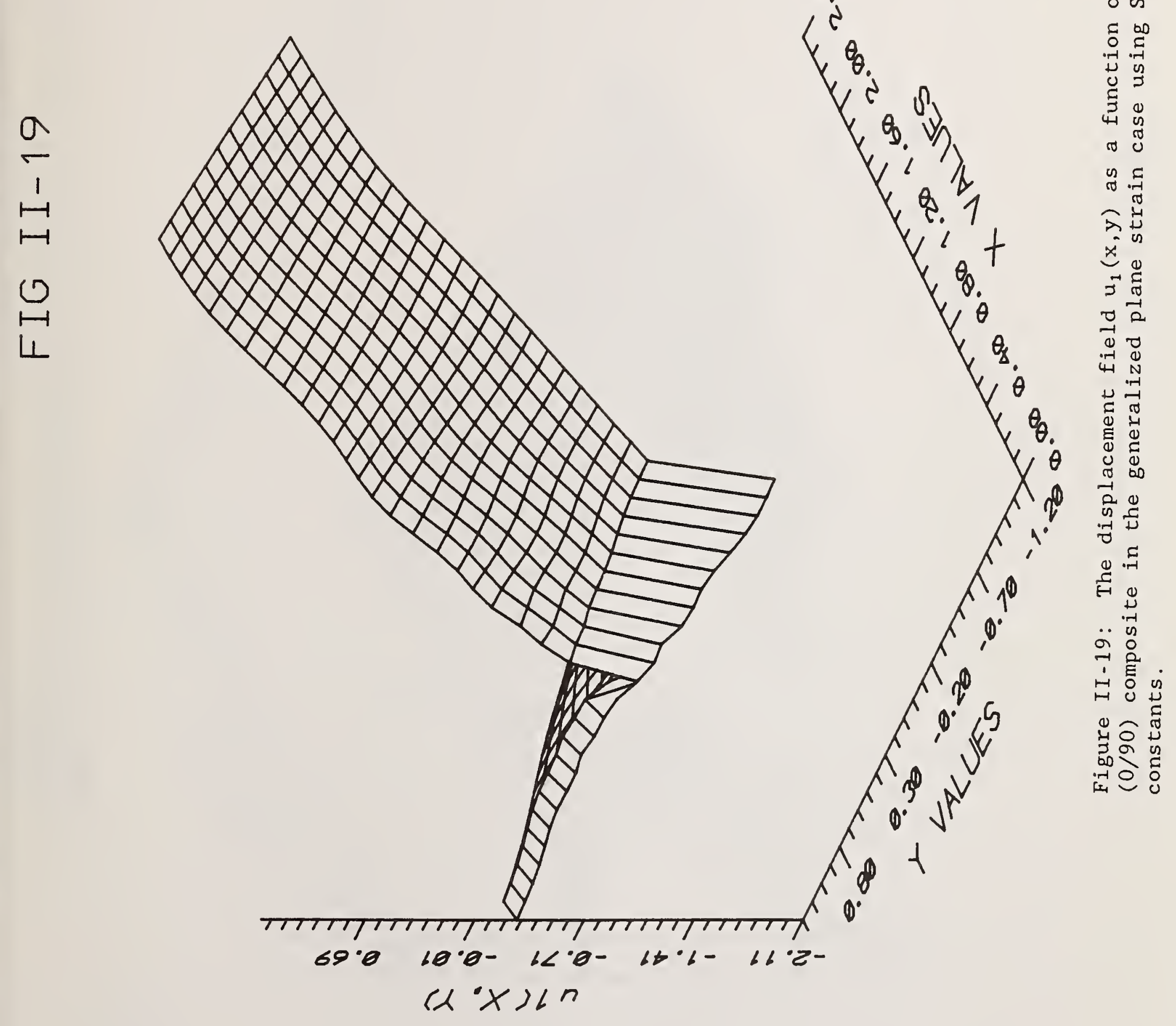




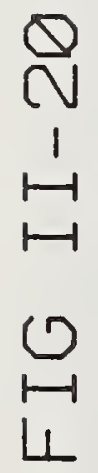

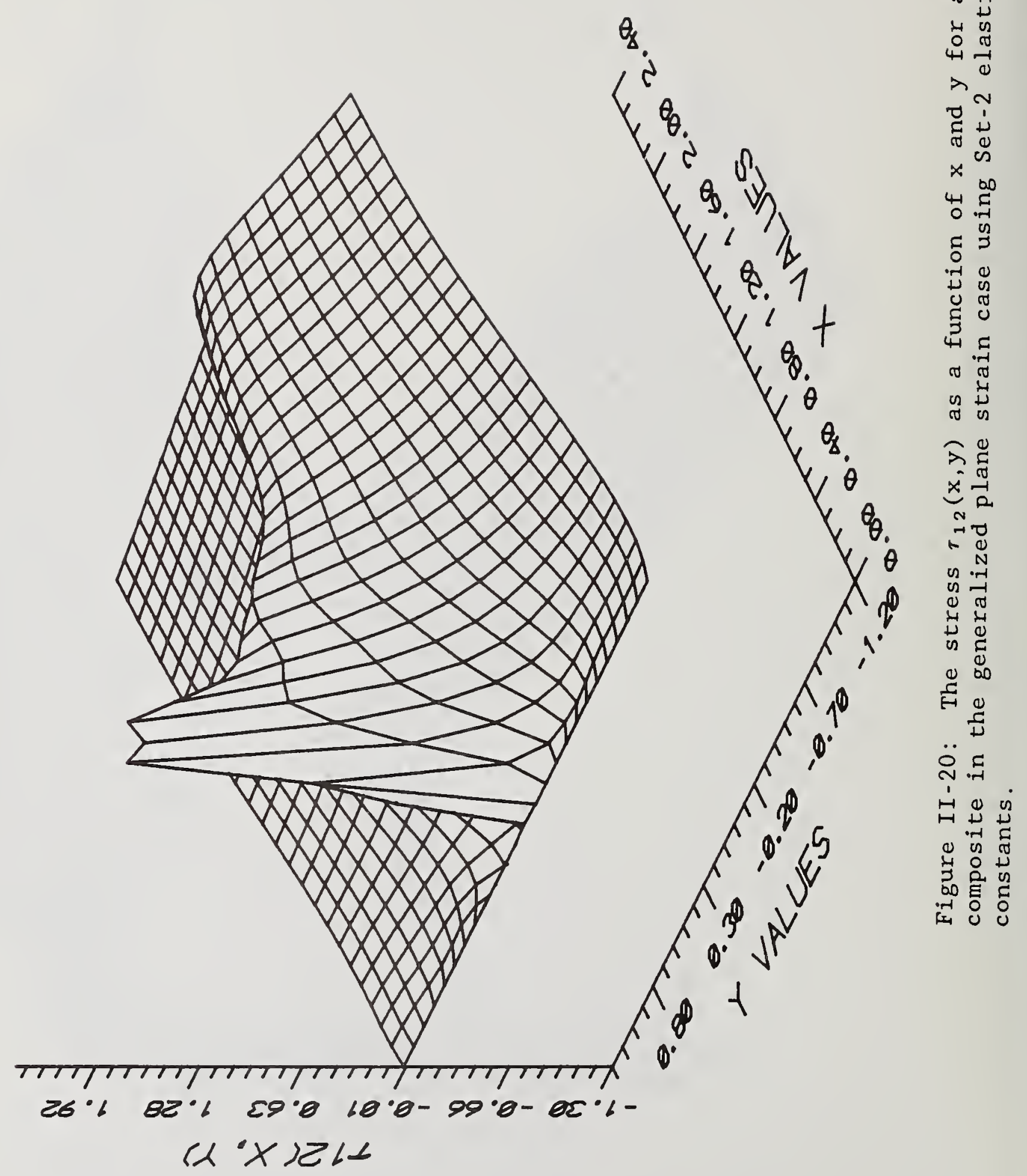




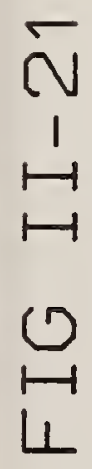

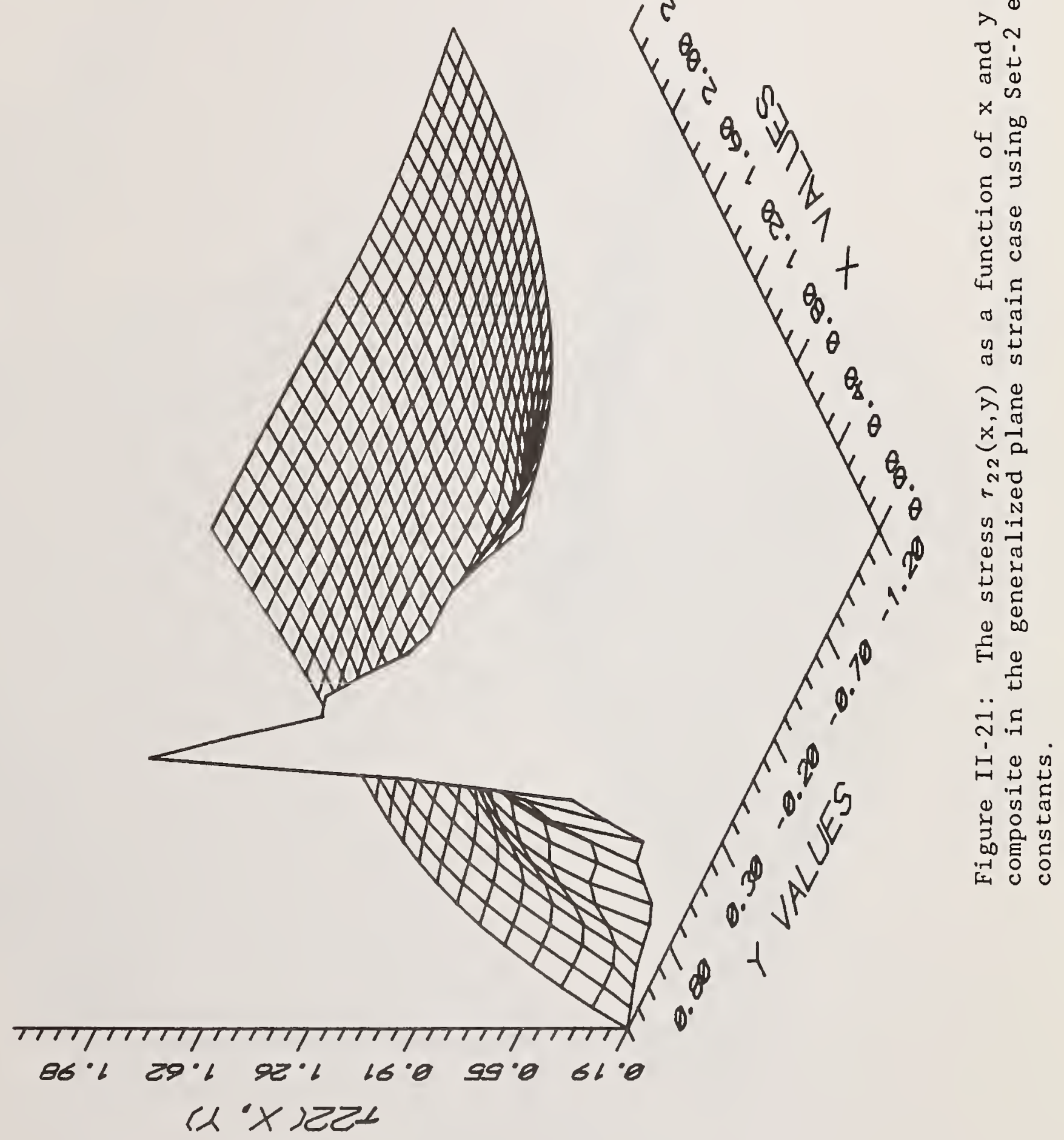




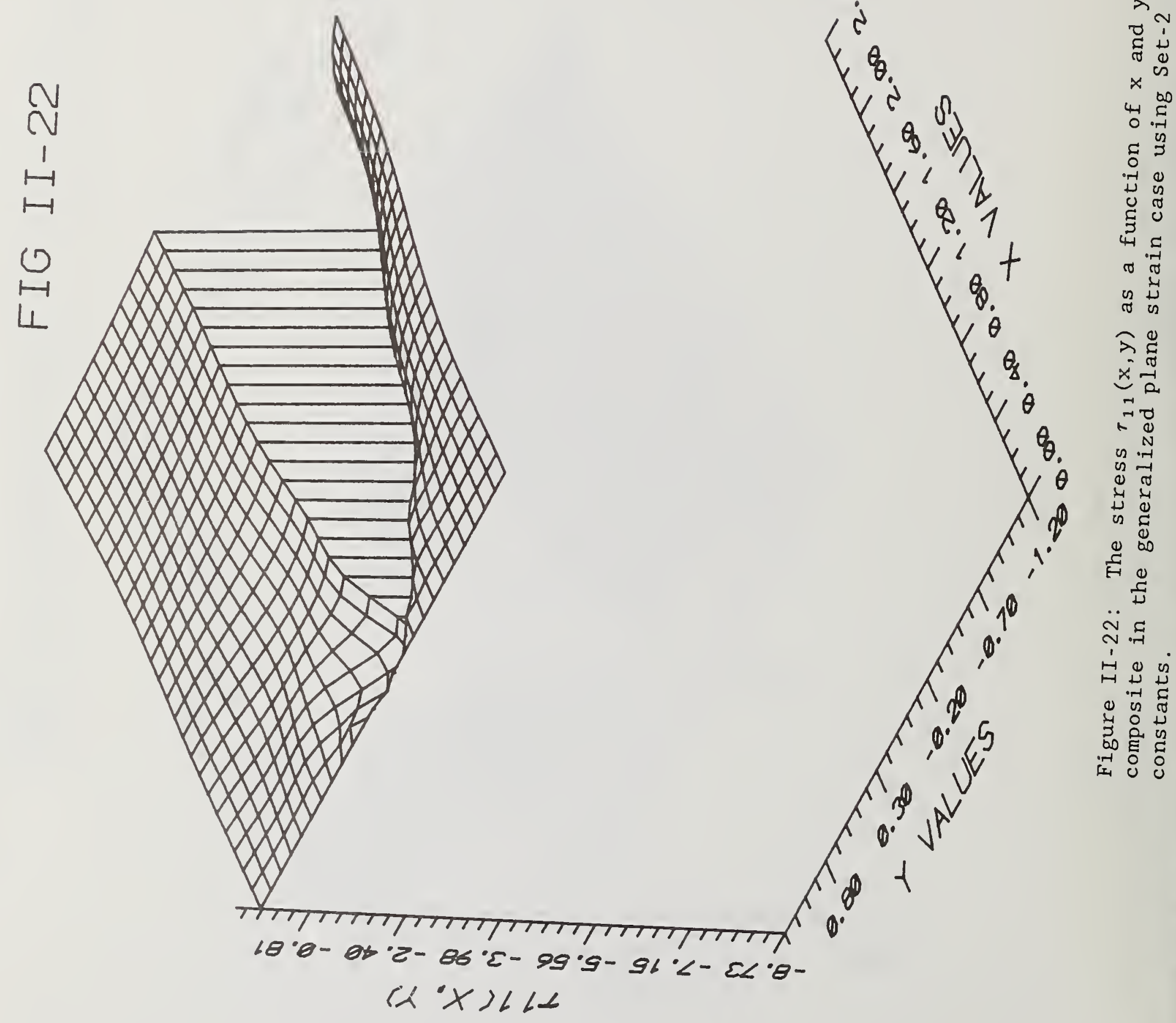


$M$
$N$
1
$\mapsto$
1
0
$H$

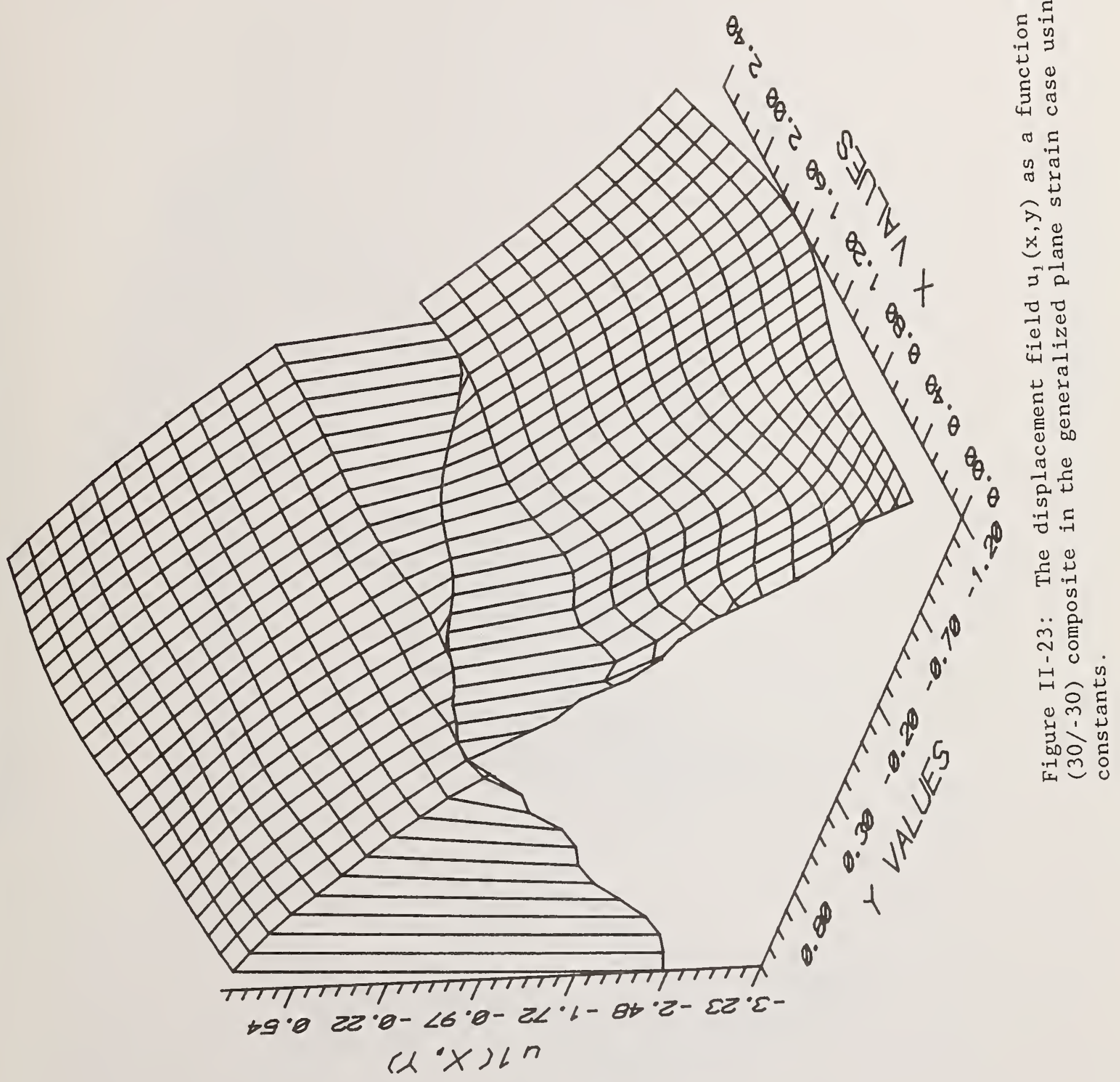




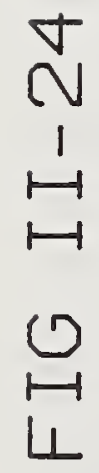

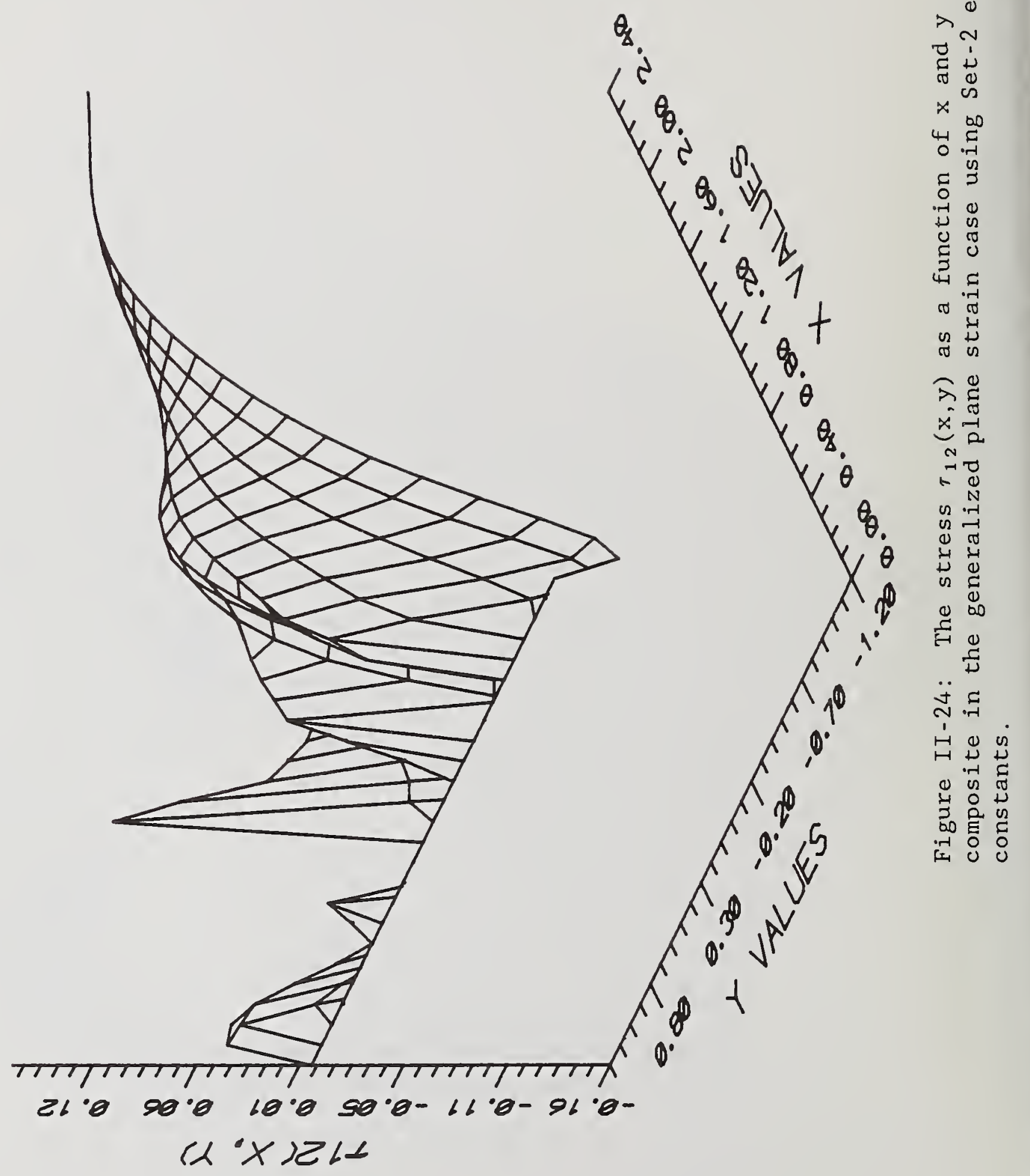




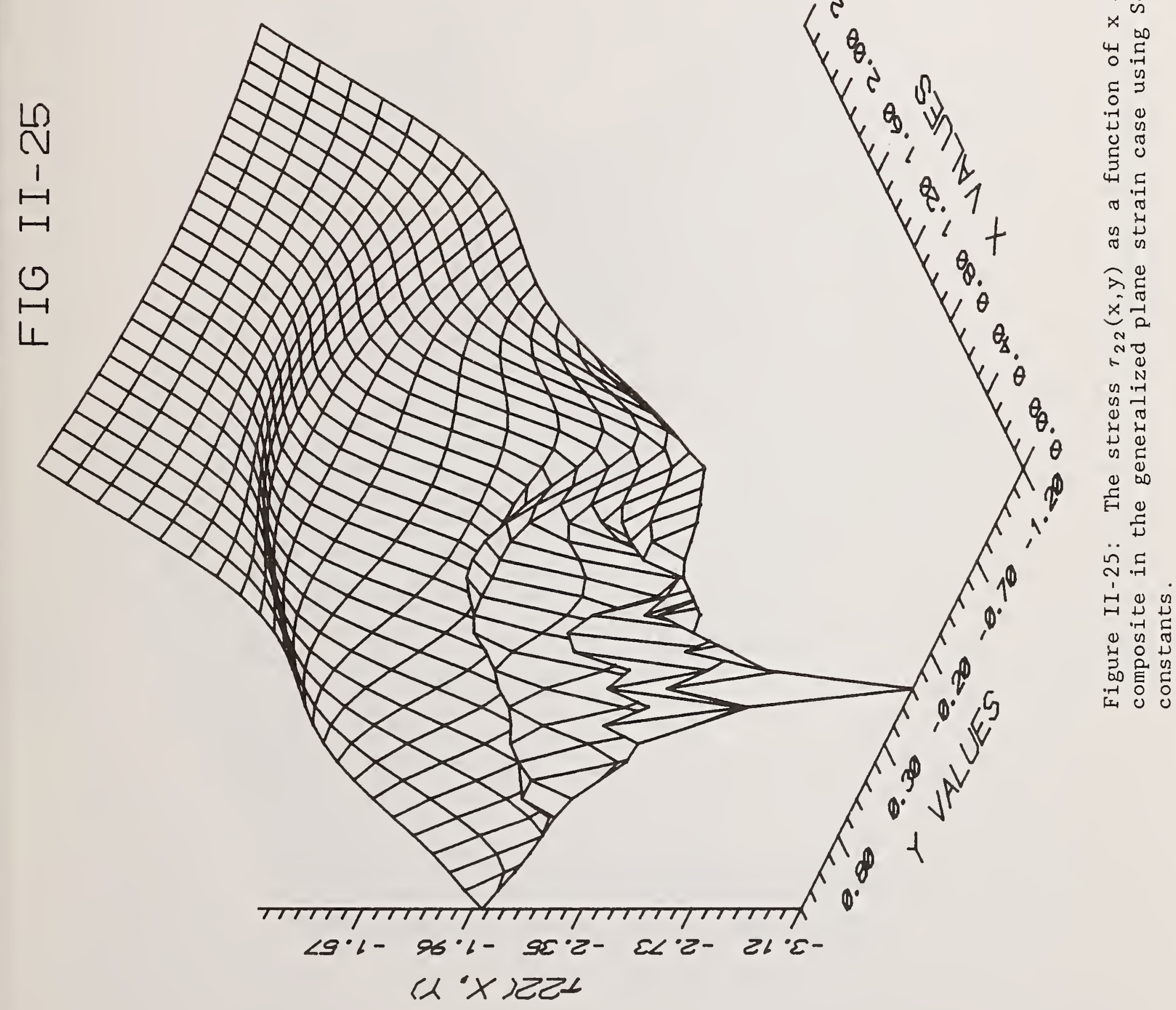




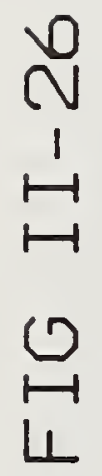

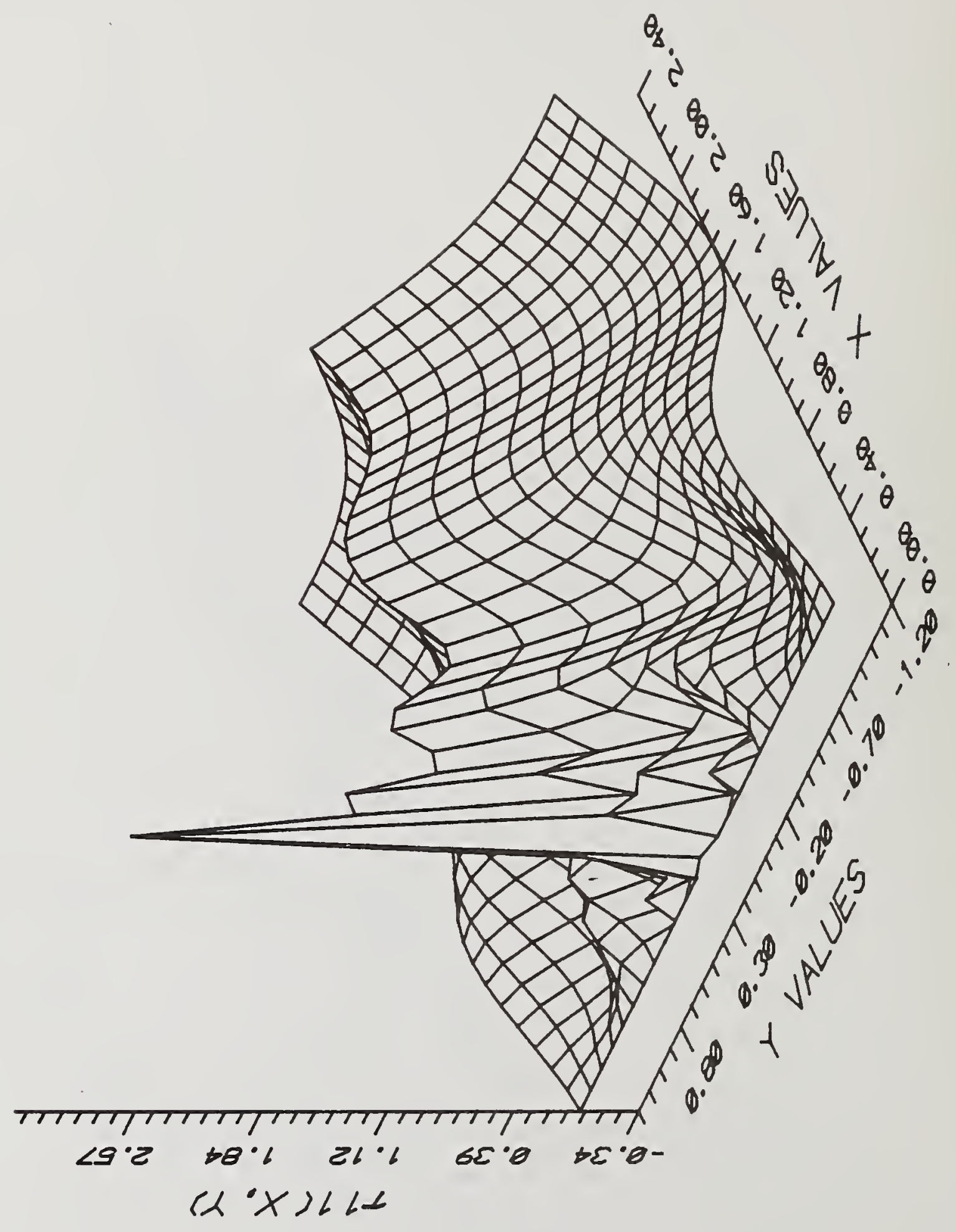

0
1
0
1

का 0

$\begin{array}{ll}4 & 2 \\ 0 & 0\end{array}$

फ

$\lambda N$

त्र

$x$ is

4.

ह

U1

$\pi$

थ

久ิ

$x$

$=0$

$\therefore d$

ข.

ये

प्र

$\begin{array}{ll}0 & 0 \\ 0 & 0\end{array}$

E

$\rightarrow \frac{1}{1}$

$\ddot{v} ᄃ$

ข.

H D

(1) थ हี

¿ 200

పิ

I 0 


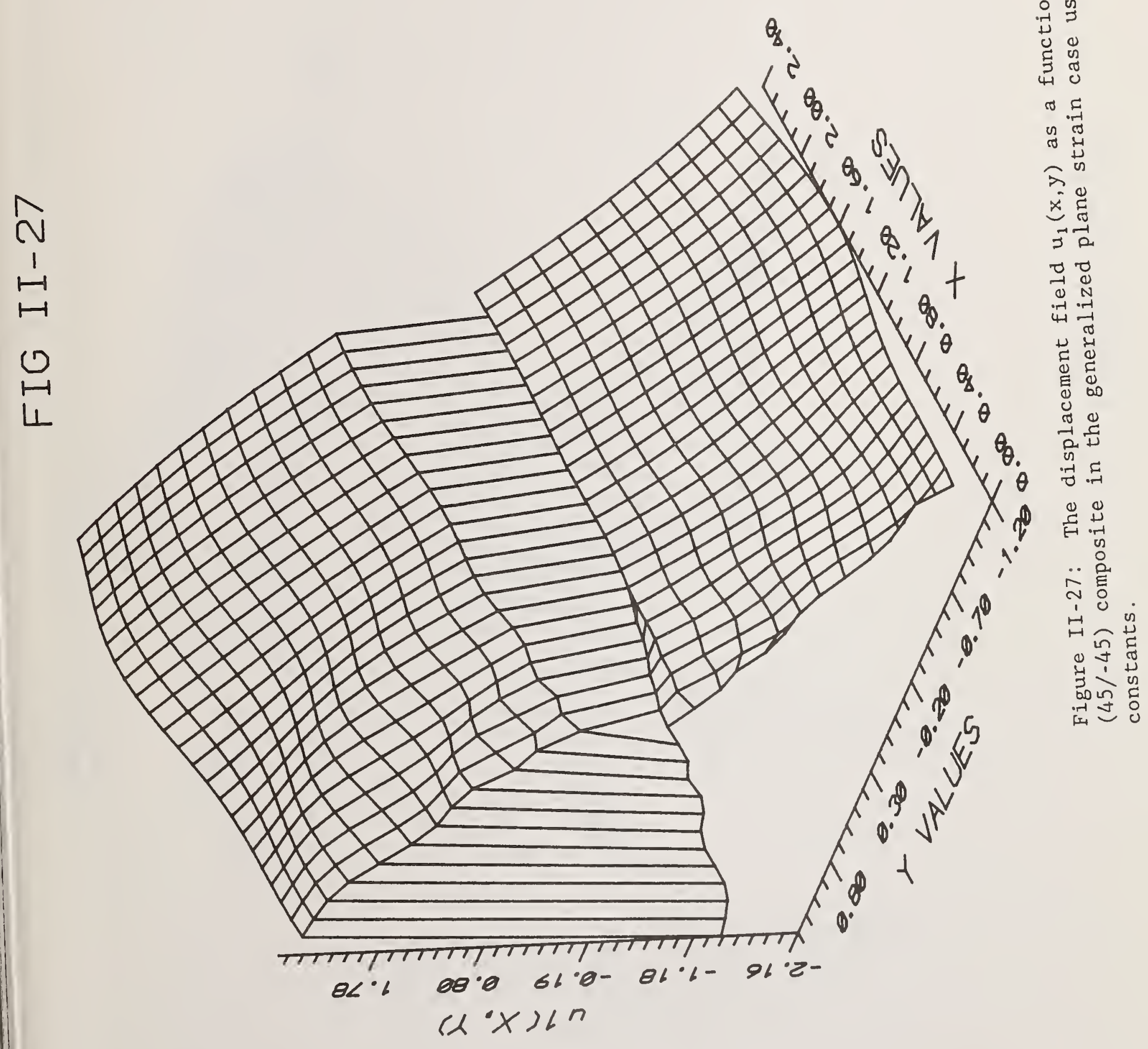



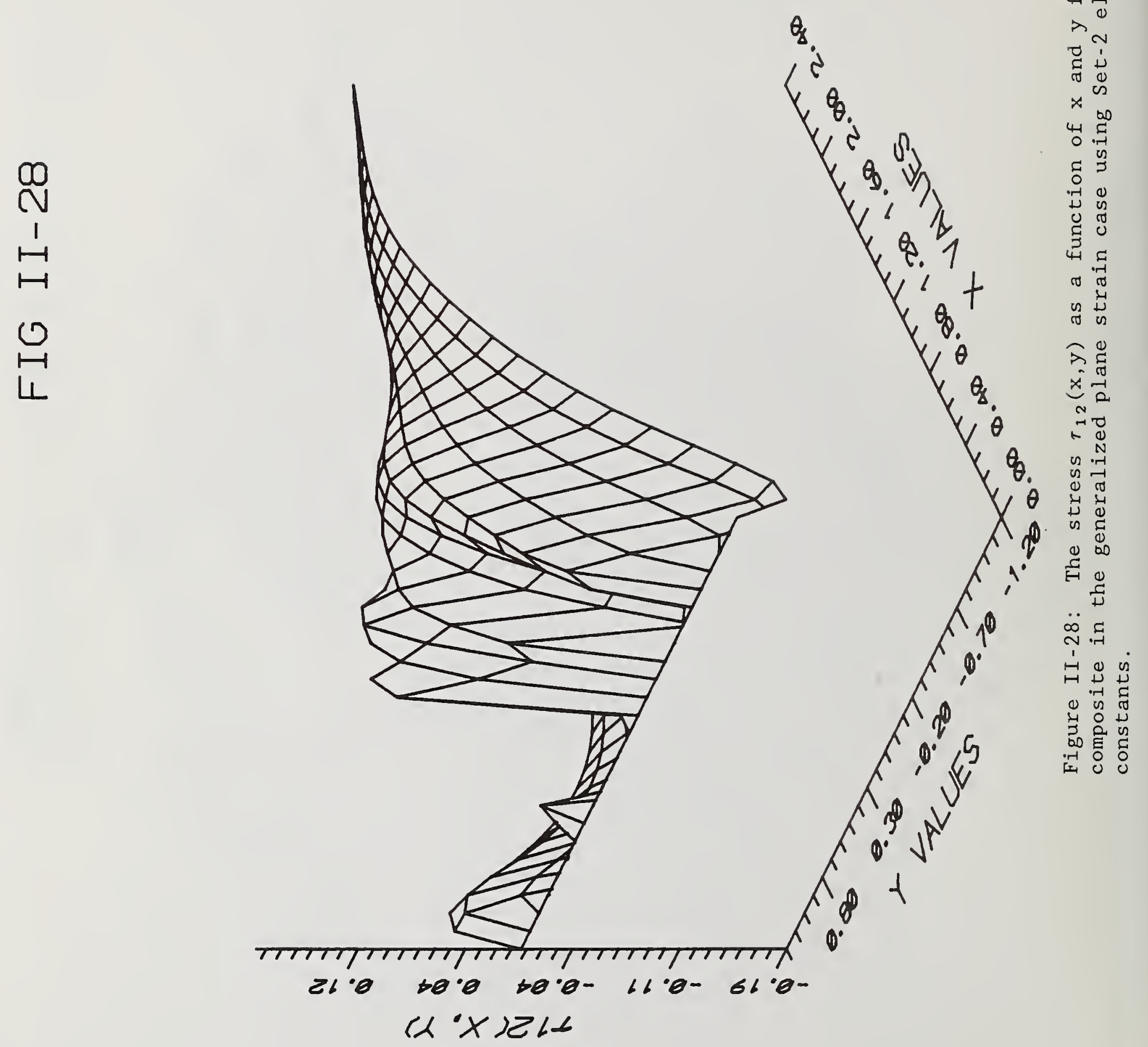


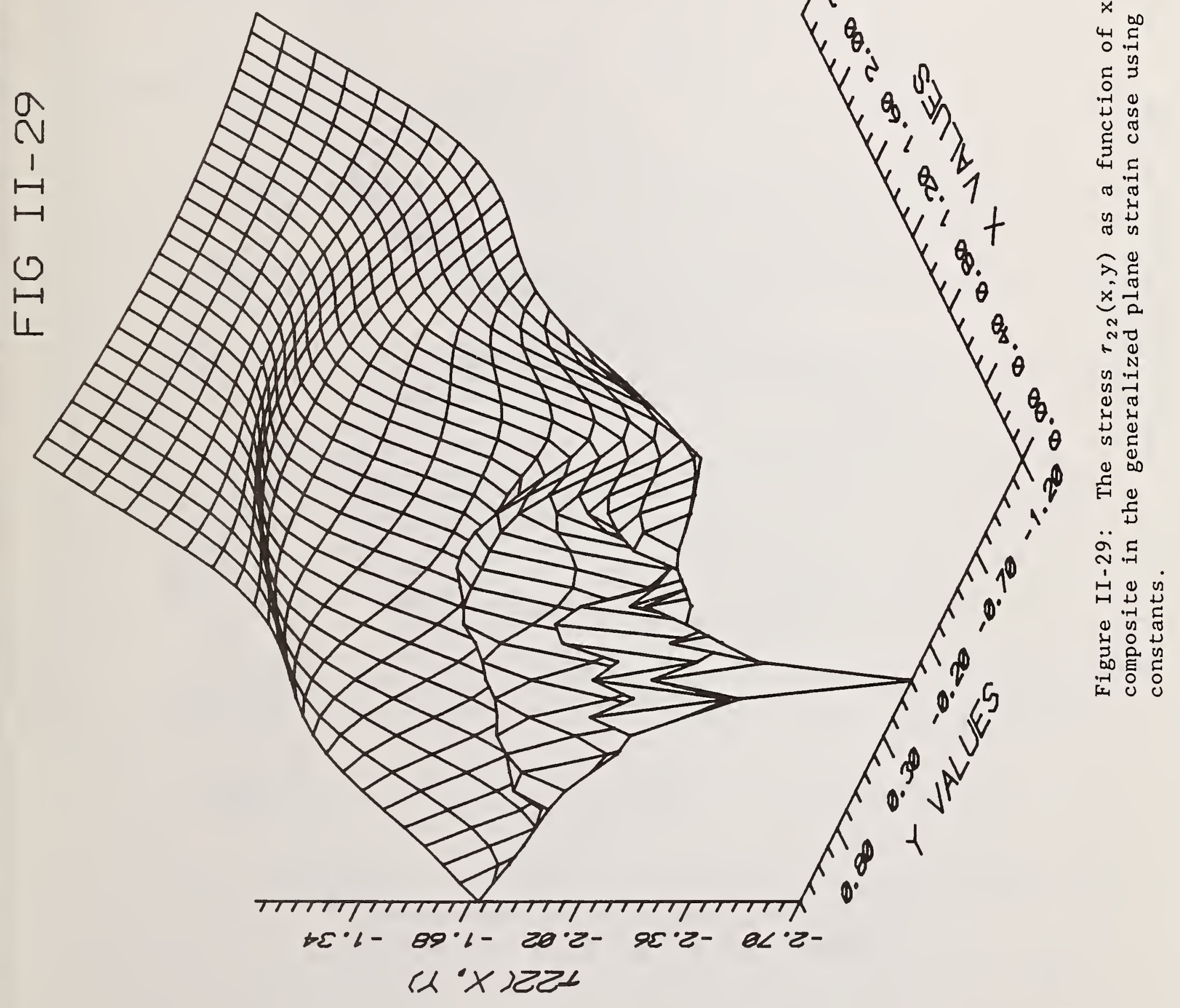


9
M
$\stackrel{4}{-4}$
0
0
4

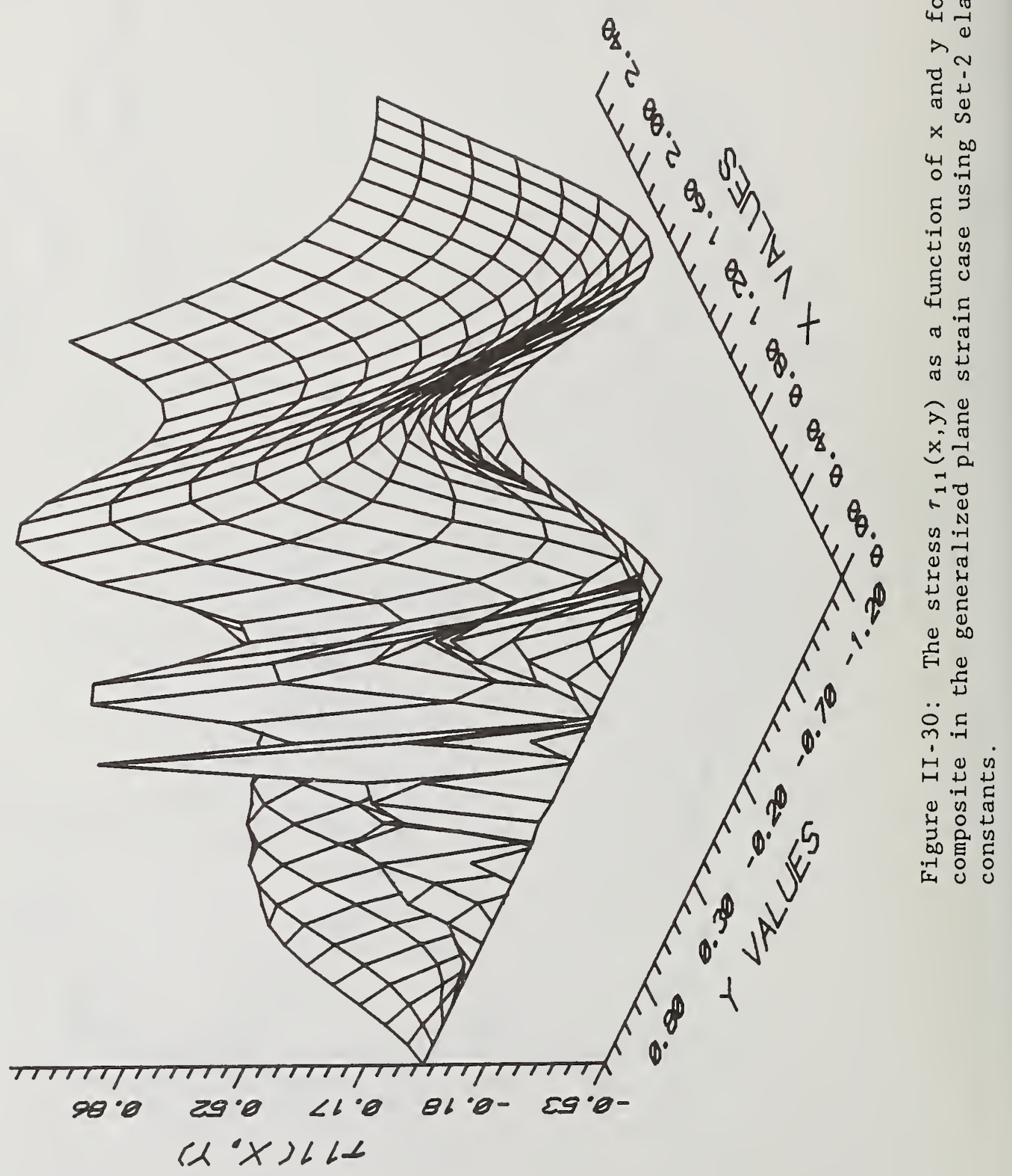




\section{Acknow ledgments}

The authors thank Dr. H.I. McHenry for his constant encouragement and support. The authors are also grateful to Drs. Robb Thomson and Ed Fuller, Jr., for reading the manuscript and many important suggestions. This work was carried out in part under a research contract between the ohio state University and the National Institute of Standards and Technology.

References (For parts I and II)

[1] R.B. Pipes and N.J. Pagano, J. Composite Materials, 4, 538 (1970).

[2] P.W. Hsu and C.T. Herakovich, J. Composite Materials, 11, 422 (1977).

[3] A.S.D. Wang and F.W. Crossman, J. Composite Materials, 11, 92 (1977).

[4] N.J. Pagano, Int. J. Solids and Structures, 14, 385 (1978).

[5] S.S. Wang and I. Choi, "Modern Developments in Composite Materials and structure," (ASME Publication; Editor J.L. Vinson), $\mathrm{p}-315$ (1979)

[6] R.L. Spilker and S.C. Chou, J. Composite Materials, 14, 2 (1980).

[7] S.G. Lekhnitskii, "Theory of Elasticity of an Anisotropic Elastic Body," (translated by P. Fern), Holden-Day, San Francisco (1963).

[8] Dempsey, J.P. and G.B. Sinclair, J. Elasticity, 9, 373 (1979).

[9] T.C.T. Ting and S.C. Chou, Int. J. Solids and Structures, 17, 1057 (1981).

[10] R.I. Zwiers, T.C.T. Ting, and R.L. Spilker, J. Appl. Mechanics, 49,561 (1982). 
[11] T.C.T. Ting and S.C. Chou, "Fracture of Composite Materials"; (Proceedings of the II U.S.A.-U.S.S.R. Symposium, Lehigh University, Bethlehen, PA, March 9-12, 1981; Edited by G.C. Sih and V.P. Tamuzs); p265 (1982).

[12] N.J. Pagano and R.B. Pipes, J. Comp. Mat.; 7, 679 (1973).

[13] R.D. Kriz, "Effect of Material Properties on Interlaminar Stresses in Angle-Ply Composite Laminates"; Report No. VPI-77-16, Virginia Polytechnic Institute and state Univirsity, Blacksburg, VA (1977). (A copy of this report can be obtained from the authors.)

[14] H.K. Stolarski and M.Y.M. Chiang, Int. J. Solids structures; $\underline{25}, 75$ (1989).

[15] C.E. Bakis and W.W. Stinchcomb; Composite Materials: Fatigue and Fracture; ASTM STP-907 (Editor H.T. Hahn; American Society for Testing and Materials); p 314 (1986).

[16] A.H. England, J. Appl. Mechanics, 32, 400 (1965).

[17] J.R. Rice and G.C. Sih, J. Appl. Mechanics, 32, 418 (1965).

[18] V.K. Tewary, R.H. Wagoner and J.P. Hirth, J. Materials Research, 4 , 113 (1989).

[19] V.K. Tewary, R.H. Wagoner and J.P. Hirth, J. Materials Research, 4, 124 (1989).

[20] N.I. Muskhelishvili, "Singular Integral Equations," (Noordhoff, Groningen) (1977).

[21] N.P. Vekua, "Systems of Singular Integral Equations," (Noordhoff, Groningen) (1977).

[22] A.N. Stroh; J. Math. Phys.; 41, 77 (1962).

[23] J.E. Sinclair and J.P. Hirth, J. Phys. F (Metal Physics), $\underline{5}$, 236 (1975).

[24] W. Bollman, "Crystal Defects and Crystalline Interfaces," (Springer-Verlag, New York) (1970).

[25] V.K. Tewary, R.M. Thomson and E.R. Fuller, Jr, J. Materials Research, 4,309 (1989). 
Effect of a Free Surface on Stress Distribution in a Bimaterial Composite 5. AUTHOR(S)

V.K. Tewary, R.D. Kriz

6. PERFORMING ORGANIZATION (IF JOINT OR OTHER THAN NIST, SEE INSTRUCTIONS)

U.S. DEPARTMENT OF COMMERCE

MATIONAL INSTITUTE OF STANDARDS AND TECHNOLOQY

BOULDER, COLORADO $80303-3328$

\section{CONTRACT/GRANT NUMBER \\ 8. TYPE OF REPORT AND PERIOD COVERED \\ Final}

9. SPONSORING ORQAMIZATION MAME AND COMPLETE ADDRESS (STREET, CITY, STATE, ZIP)

Same as item \#6

10. SUPPLEMENTARY NOTES 11. ABSTRACT (A 200-WORD OR LESS FACTUAL SUMMARY OF MOST SIQNIFICANT INFORMATION. IF DOCUMENT INCLUDES A SIGNIFICANT BIBLOQRAPHY OR
UTERATURE SURVEY, MENTION IT HERE.)

This paper is in two parts. In part I, the elastic plane-strain Green's function is calculated for an anisotropic bimaterial composite solid containing a free surface normal to the interface. An exact integral representation is obtained for the Green's function, which is evaluated numerically. The integral is also evaluated analytically, which gives a series representation for the Green's function. For illustration, the formalism is applied to the antipane-strain problem and the plane-strain problem in a cubic solid containing a $5-5$ grain boundary. In part II, the Green's function is extended to the case of generalized plane strain and applied to calculate the stress field in a cubic solid containing a $\Sigma-5$ boundary and to fiber-reinforced composites with various fiber orientations subjected to an out-of-plane load.

It is found that, as predicted by earlier authors, the stress is singular at the intersection of the free surface and the Interface in both plane strain and generalized plane-strain case. These singularities in the stress field associated with the presence of the fret surface are identified and discussed. The singularies in the stress field are found to be of the type $\mathrm{r}^{-\delta}, \ln (\mathrm{r})$ as well as containing higher powers of $\ln (\mathrm{r})$, where $\delta$ is between 0 and 1 and $r$ is the radial distance from the intersection of the free suriace and the interface. In addition, it is found that, in general, the stress field will also contain an oscillatory factor of the type exp[lgln(r)], where $g$ is a conscant that depends upon the material parameters cf the two solids. However, for the case of the generalized plane strain in fiber-reinforced composites, the factor $g$ is almost 0 so that the oscillatory behavior is absent. Part I also gives a simple and convenient method for solving an inhomogeneous generalized vector Hilbert problem, which contains a nonsingular kernel in addition to the usual singular Hilbert kernel. The solution of this Hilbert problem is required for obtaining the Green's function in the present case, as well as many other cases dealing with the stress analysis of composite materials containing surfaces, interfaces, and cracks.

The solution of the Hilbert problem is obtained by using a complex transform of the type $y$ 19-0.5, where $y$ and $q$ are variables on the real axis. It is shown that this function is an eigenfunction of the Hilbert operator and is orthogonal for $y$, varying in the razge between 0 and \pm w and is butween $-\infty$ to $+\infty$.

12. KEY WORDS (6 TO 12 ENTRIES; ALPHABETICAL ORDER; CAPITALZE ONLY PROPER NAMES; AND SEPARATE KEY WORDS BY SEMICOLONS) antiplane strain; bimaterial composites; fiber reinforced composites; free edge effect; fres surface; generalizcd plaic strain; Greci's furction; Hilbert problen; plane strain; singularities in stress.

\section{5}

\section{PRICE}






\section{NISTT Technical Publications}

\section{Periodical}

Journal of Research of the National Institute of Standards and Technology-Reports NIST research and development in those disciplines of the physical and engineering sciences in which the Institute is active. These include physics, chemistry, engineering, mathematics, and computer sciences. Papers cover a broad range of subjects, with major emphasis on measurement methodology and the basic technology underlying standardization. Also included from time to time are survey articles on topics closely related to the Institute's technical and scientific programs. Issued six times a year.

\section{Nonperiodicals}

Monographs-Major contributions to the technical literature on various subjects related to the Institute's scientific and technical activities.

Handbooks-Recommended codes of engineering and industrial practice (including safety codes) developed in cooperation with interested industries, professional organizations, and regulatory bodies.

Special Publications-Include proceedings of conferences sponsored by NIST, NIST annual reports, and other special publications appropriate to this grouping such as wall charts, pocket cards, and bibliographies.

Applied Mathematics Series-Mathematical tables, manuals, and studies of special interest to physicists, engineers, chemists, biologists, mathematicians, computer programmers, and others engaged in scientific and technical work.

National Standard Reference Data Series-Provides quantitative data on the physical and chemical properties of materials, compiled from the world's literature and critically evaluated. Developed under a worldwide program coordinated by NIST under the authority of the National Standard Data Act (Public Law 90-396). NOTE: The Journal of Physical and Chemical Reference Data (JPCRD) is published quarterly for NIST by the American Chemical Society (ACS) and the American Institute of Physics (AIP). Subscriptions, reprints, and supplements are available from ACS, 1155 Sixteenth St., NW., Washington, DC 20056.

Building Science Series-Disseminates technical information developed at the Institute on building materials, components, systems, and whole structures. The series presents research results, test methods, and performance criteria related to the structural and environmental functions and the durability and safety characteristics of building elements and systems.

Technical Notes-Studies or reports which are complete in themselves but restrictive in their treatment of a subject. Analogous to monographs but not so comprehensive in scope or definitive in treatment of the subject area. Often serve as a vehicle for final reports of work performed at NIST under the sponsorship of other government agencies.

Voluntary Product Standards-Developed under procedures published by the Department of Commerce in Part 10, Title 15, of the Code of Federal Regulations. The standards establish nationally recognized requirements for products, and provide all concerned interests with a basis for common understanding of the characteristics of the products. NIST administers this program as a supplement to the activities of the private sector standardizing organizations.

Consumer Information Series-Practical information, based on NIST research and experience, covering areas of interest to the consumer. Easily understandable language and illustrations provide useful background knowledge for shopping in today's technological marketplace.

Order the above NIST publications from: Superintendent of Documents, Government Printing Office,

Washington, DC 20402.

Order the following NIST publications-FIPS and NISTIRs-from the National Technical Information Service, Springfield, VA 22161.

Federal Information Processing Standards Publications (FIPS PUB)-Publications in this series collectively constitute the Federal Information Processing Standards Register. The Register serves as the official source of information in the Federal Government regarding standards issued by NIST pursuant to the Federal Property and Administrative Services Act of 1949 as amended, Public Law 89-306 (79 Stat. 1127), and as implemented by Executive Order 11717 (38 FR 12315, dated May 11 , 1973) and Part 6 of Title 15 CFR (Code of Federal Regulations).

NIST Interagency Reports (NISTIR)-A special series of interim or final reports on work performed by NIST for outside sponsors (both government and non-government). In general, initial distribution is handled by the sponsor; public distribution is by the National Technical Information Service, Springfield, VA 22161, in paper copy or microfiche form. 
U.S. Department of Commerce

National Institute of Standards and Technology

Gaithersburg, MD 20899

Official Business

Penalty for Private Use $\$ 300$ 